Printed at the Mathematical Centre, 49, 2e Boerhaavestraat, Amsterdam.

The Mathematical Centre, founded the 11-th of February 1946, is a nonprofit institution aiming at the promotion of pure mathematics and its applications. It is sponsored by the Netherlands Government through the Netherlands Organization for the Advancement of Pure Research (Z.W.O). 
MATHEMATICAL CENTRE TRACTS 77

\section{ASYMPTOTIC OPTIMALITY OF LIKELIHOOD RATIO TESTS IN EXPONENTIAL FAMILIES}

W.C.M. KALLENBERG

MATHEMATISCH CENTRUM AMSTERDAM 1978 
AMS (MOS) subject classification scheme (1970): 60F10, 62E20, 62F05, 62F20 


\section{PREFACE}

In this treatise optimality of Likelihood Ratio (LR) tests in exponential families is investigated with respect to two criterions. The first criterion concerns the shortcoming of a test. For simple null hypotheses OOSTERHOFF and VAN ZWET (1970) have studied the shortcoming of the LR test in the multinomial case. Their results can be extended both to composite null hypotheses and to much more general classes of distributions: exponential families. In this study testing problems are considered where the level of significance tends to zero as the sample size, $n$, tends to infinity. It turns out that unöer some conditions the LR test is a good test in the sense that its shortcoming tends to zero uniformly over parts of the parameter space.

For the second criterion the concept of Bahadur deficiency is introduced. Roughly speaking a sequence of tests is deficient in the sense of Bahadur of order $O\left(h_{n}\right)$ - or $O\left(h_{n}\right)$ - at some parameter point $\theta$ if the additional number of observations necessary to obtain the same power at $\theta$ as the optimal test is of order $O\left(h_{n}\right)$ - or $O\left(h_{n}\right)-;$ here $h_{n}$ is a positive function on $\mathbf{N}$. In BAHADUR (1971) it is shown that under rather strong conditions the LR test is efficient in the sense of Bahadur, which corresponds to Bahadur deficiency of order $O(n)$ as $n \rightarrow \infty$. This result may be regarded as a "first order" result. A deeper analysis yields that in typical cases the Bahadur deficiency is in fact of order $O(\log n)$. The introduction of Bahadur deficiency in cases of optimal Bahadur efficiency runs parallel to the consideration of Pitman deficiency introduced by Hodges and Lehmann.

A basic tool in both approaches to optimality are theorems on probabilities of large deviations. An important part of this study is devoted to the derivation of such results.

It is a pleasure for me to thank prof.dr. J. Oosterhoff for his stimulating advice and his continuous interest in this work.

I thank the Mathematical Centre for the opportunity to publish this monograph in their series Mathematical Centre Tracts and all those at the Mathematical Centre who have contributed to its technical realization. 
Preface . . . . . . . . . . . . . . . . . . v

Contents. . . . . . . . . . . . . . . . . . vrr

I. INTRODUCTION ..................... 1

1.1. Classical results about the likelihood ratio test . . . . 1

1.2. LR tests in exponential families .......... 5

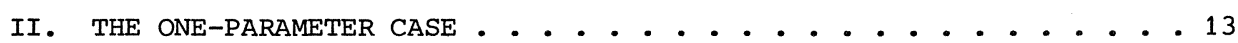

2.1. Introduction. ................. 13

2.2. Preliminaries ................. 14

2.3. Relation between $\alpha_{n}$ and $d_{n}$............. 16

2.4. The MP test of $\mathrm{H}_{0}$ against a simple alternative. . . . . 20

2.5. The main theorem. ............... 21

2.6. Some particular cases . . . . . . . . . . . 26

2.7. Uniform convergence on compact subsets of int $\theta . . . .31$

2.8. Pointwise convergence . . . . . . . . . . . . 34

III. THE k-PARAMETER CASE . . . . . . . . . . . . . 37

3.1. Introduction. . . . . . . . . . . . . . 37

3.2. A generalization of a theorem of Efron and Truax. . . . . 39

3.3. The null hypothesis contained in a compact subset of int $\theta . .46$

3.4. The alternative hypothesis contained in a compact subset

of int $\theta$. . . . . . . . . . . . . . . 53

3.5. Uniform convergence on compact subsets of int $\theta . . . .61$

3.6. The k-dimensional normal distribution with known covariance

matrix................. . . 65

3.7. The multinomial distribution. ............. 66

3.8. The alternative hypothesis a proper subset of the complement of the null hypothesis. . . . . . . . . . . 72

IV. RELATIONS BETWEEN SHORTCOMING AND BAHADUR DEFICIENCY . . . . . 77

4.1. A fundamental theorem . . . . . . . . . . . 77

4.2. Examples. . . . . . . . . . . . . . 87 
V. BAHADUR DEFICIENCY OF THE LIKELIHOOD RATIO TEST . . . . . . . . 91

5.1. Introduction . . . . . . . . . . . . . 91

5.2. The one-dimensional case . . . . . . . . . . . 94

5.3. The k-dimensional case . . . . . . . . . . 107

REFERENCES. . . . . . . . . . . . . . . . 125 


\section{CHAPTER I}

\section{INTRODUCTION}

\subsection{CLASSICAL RESULTS ABOUT THE LIKELIHOOD RATIO TEST}

In this section we present a brief survey of classical results about likelihood ratio (LR) tests in general testing problems. We begin by introducing some notation that will be used throughout this study.

Let $X$ be a set of points $x$ and $B$ a $\sigma$-field of subsets of $X . \theta$ is an index set of points $\theta$ and, for each $\theta$ in $\theta, P_{\theta}$ is a probability measure on $B$. The pair $(X, B)$ is to be thought of as the range space of an observation $X$ whose distribution is determined by the unknown parameter $\theta$. Let $S=X_{1} \times X_{2} \times \ldots$ $=\left\{\left(x_{1}, x_{2}, \ldots\right) ; x_{i} \in X_{i}, i=1,2, \ldots\right\}$ be the infinite product space of $a$ sequence of replicates $X_{i}$ of $X$ with product $\sigma$-field $A$. On $(S, A) P_{\theta}$ will denote the product measure. Define $s(s)=s$ and $x_{n}(s)=x_{n}$ for $s=\left(x_{1}, x_{2}, \ldots\right) \in S$ $(\mathrm{n}=1,2, \ldots)$. Then $\mathrm{x}_{1}, \mathrm{x}_{2}, \ldots$ are independent identically distributed (i.i.d.) random variables with distribution $P_{\theta}$. The family of distributions $\left\{P_{\theta} ; \theta \in \theta\right\}$ of $x_{1}$ is assumed to be a dominated parametric family with densities $\left\{p_{\theta}(x) ; \theta \in \theta\right\}$ with respect to a $\sigma$-finite measure $\mu$.

For the testing problem of a simple hypothesis $\theta=\theta_{0}$ against a simple alternative $\theta=\theta_{1}$ with available observations $x_{1}, \ldots, x_{n}$ NEYMAN and PEARSON $(1928,1933)$ have determined a most powerful (MP) test by using a test statistic based on the ratio of the densities of $x_{1}, \ldots, x_{n}$ under $\theta_{0}$ and $\theta_{1}$. They also presented in [16] a natural extension of this test to composite hypotheses: the LR test. First let us describe this LR procedure.

Let $\theta_{0}$ be an arbitrary subset of $\theta$. The size- $\alpha$ LR test of the null hypothesis $\theta_{0}$ against $\theta_{1}=\theta-\theta_{0}$, based on $n$ observations $x_{1}, \ldots, x_{n}$, is defined by

$$
\phi_{n}^{L R}(s)= \begin{cases}1 & > \\ \delta_{n} \text { if } T_{n}^{L R}(s) & =d_{n^{\prime}} \\ 0 & <\end{cases}
$$


where

$(1.1 .2)$

$$
\begin{aligned}
T_{n}^{L R}(s) & =T_{n}^{L R}\left(\left(x_{1}, x_{2}, \ldots\right)\right)= \\
& =-n^{-1} \log \left\{\frac{\sup _{\theta_{0} \in \Theta_{0}} \Pi_{i=1}^{n} P_{\theta_{0}}\left(x_{i}\right)}{\sup _{\theta \in \Theta} \pi_{i=1}^{n} p_{\theta}\left(x_{i}\right)}\right\}
\end{aligned}
$$

with the convention $T_{n}^{L R}(s)=0$ if the numerator and denominator in (1.1.2) are both 0 or $\infty$. The constants $d_{n}$ and $\delta_{n}$ appearing in (1.1.1) are determined by

$$
\sup _{\theta_{0} \in \Theta_{0}} \mathrm{E}_{\theta_{0}} \phi_{\mathrm{n}}^{\mathrm{LR}}(\mathrm{S})=\alpha, \quad 0<\alpha<1, \mathrm{n}=1,2, \ldots
$$

It will be assumed that the densities $p_{\theta}$ are so smooth that $\phi_{n}^{L R}$ is a measurable function on $(S, A)$. Conditions to ensure this property are given by WITTING and NÖLLE (1970) p.93. Note that the test statistic $\mathrm{T}_{\mathrm{n}}^{\mathrm{LR}}$ is related to the maximum likelihood estimators of $\theta$ over the spaces $\theta$ and $\theta_{0}$. Hence one would expect the properties of the test statistic to be intimately tied up with properties of maximum likelihood estimators.

Intuitively $\phi_{n}^{L R}$ is a good test. For, if we are comparing the "plausibility" of one value of $\theta$ to another, given the sample $\left(x_{1}, \ldots, x_{n}\right)$, we would be inclined to choose that value of $\theta$ for which the density has the larger value. Thus, if we can obtain an appreciably larger value of the density if $\theta$ runs through the entire parameter space $\theta$ than we get by varying $\theta$ over the set $\theta_{0}$, our intuition will assess the evidence as strongly in favour of the alternative hypothesis. However, there are examples where $\phi_{n}^{L R}$ is less powerful than the trivial test $\phi \equiv \alpha$ (cf. WITTING and NÖLLE (1970) p.93). Now suppose $\theta \subset \mathbb{R}^{k}$. If $\theta_{0}$ is a single point or a h-dimensional linear subspace of $\theta(\mathrm{h}<\mathrm{k})$ WILKS (1938) has derived under fairly general conditions that for each $\theta_{0} \in \Theta_{0}$ the asymptotic distribution of $2 \mathrm{nT}_{\mathrm{n}}^{\mathrm{LR}}$ is a chisquare distribution with $\mathrm{k}$ or $\mathrm{k}-\mathrm{h}$ degrees of freedom, respectively. Slightly more general results have been derived by WALD (1943) and can also be found in WITTING and NÖLLE (1970), pp.94-96. (Note that witting and Nolle incorrectly suggest that under their conditions also $\sup _{\theta_{0} \epsilon \Theta_{0}} P_{\theta_{0}}\left(2 \mathrm{nT}{ }_{\mathrm{n}}^{\mathrm{LR}} \geq \chi_{\mathrm{k}-\mathrm{h} ; 1-\alpha}^{2}\right)$ $\rightarrow \alpha$ as $\mathrm{n} \rightarrow \infty$, where $\chi_{\mathrm{k}-\mathrm{h} ; 1-\alpha}^{2}$ is the upper $\alpha$-quantile of a chi-square distribution with $\mathrm{k}-\mathrm{h}$ degrees of freedom.) Moreover, wilks proved that $\phi_{\mathrm{n}}^{\mathrm{LR}}$ is a consistent test if the null hypothesis is a linear subspace of $\theta$.

For simple hypotheses $\theta_{0}=\left\{\theta_{0}\right\}$ WALD (1943) proves the following 
optimality properties of LR tests. Let

$$
A_{n}=\left\{\theta ;\left(\theta-\theta_{0}\right) \cdot J\left(\theta-\theta_{0}\right)=a_{n}\right\}, \quad n=1,2, \ldots,
$$

where $\lim _{n \rightarrow \infty} n a_{n}=a, 0<a<\infty$, and $J$ is the Fisher information matrix at $\theta_{0}$. Then the LR test has asymptotic best average power (with respect to an appropriate weight function) over the surfaces $A_{n}$ of contiguous alternatives. Moreover, the LR test is asymptotically most stringent for any sequence $\left\{A_{n}\right\}$. The previous optimality properties hold true for testing problems with fixed level of significance $\alpha$. During the last 15 years LR tests have been studied in the context of testing problems with vanishing level of significance $\alpha_{n}$ as $n \rightarrow \infty$. In BAHADUR (1965) it is shown that "the LR statistic is an optimal sequence in terms of exact stochastic comparison". This stochastic comparison introduced in BAHADUR (1960) leads to an optimality criterion, now called Bahadur efficiency. We describe this concept in some detail.

Let $\left\{\phi_{n}^{\gamma} ; \gamma \in \Gamma\right\}, n=1,2, \ldots$, be a family of (randomized) tests based on $x_{1}, \ldots, x_{n}$, where $\Gamma$ is an index set with the following property: for each $\alpha$, $0<\alpha<1$, there exists one and only one $\gamma \in \Gamma$, denoted by $\gamma_{n}(\alpha)$, such that

$$
\sup _{\theta_{0} \in \Theta_{0}} E_{\theta_{0}}^{\phi_{n}^{\gamma_{n}(\alpha)}}\left(x_{1}, \ldots, x_{n}\right)=\alpha .
$$

Short for $\left\{\phi_{n}^{\gamma} ; \gamma \in \Gamma\right\}$ we often write $\left\{\phi_{n}\right\}$. For $0<\beta<1$ and $\theta \in \theta_{1}$ define

$$
\mathrm{N}_{\phi}(\alpha, \beta, \theta)=\min \left\{\mathrm{n}_{;} \mathrm{E}_{\theta} \phi_{\mathrm{m}}^{\gamma_{\mathrm{m}}(\alpha)}\left(\mathrm{x}_{1}, \ldots, \mathrm{x}_{\mathrm{m}}\right) \geq \beta, \mathrm{m} \geq \mathrm{n}\right\} \text {. }
$$

If $\left\{\tilde{\phi}_{n}^{\gamma} ; \gamma \in \tilde{\Gamma}\right\}$ is another family of tests the Bahadur efficiency of $\left\{\phi_{n}\right\}$ with respect to $\left\{\tilde{\phi}_{n}\right\}$ is defined by

$$
\mathrm{e}_{\phi, \tilde{\phi}}(\beta, \theta)=\lim _{\alpha \downarrow 0} \frac{\mathrm{N}_{\tilde{\phi}}(\alpha, \beta, \theta)}{\mathrm{N}_{\phi}(\alpha, \beta, \theta)}
$$

provided the limit exists. If $e_{\phi}, \tilde{\phi}(\beta, \theta) \geq 1$ for all families $\left\{\tilde{\phi}_{n}\right\}$ and all $\beta$, the family $\left\{\phi_{n}\right\}$ may be called efficient in the sense of Bahadur at $\theta$. In such cases the introduction of Bahadur deficiency provides further information about the performance of $\left\{\phi_{n}\right\}$. Let $N^{+}(\alpha, \beta, \theta)=$ inf $N_{\tilde{\phi}}(\alpha, \beta, \theta)$, where the infimum is taken over all families $\left\{\tilde{\phi}_{n}\right\}$. If for all $0<\beta<1$

(1.1.3) $\quad \lim _{\alpha \rightarrow 0}\left\{\mathrm{~N}_{\phi}(\alpha, \beta, \theta)-\mathrm{N}^{+}(\alpha, \beta, \theta)\right\}\left\{\mathrm{N}^{+}(\alpha, \beta, \theta)\right\}^{-\frac{1}{2}}=0$

we shall say that $\left\{\phi_{n}^{\gamma} ; \gamma \in \Gamma\right\}$ is deficient in the sense of Bahadur at $\theta$ of 
order $O\left(\mathrm{~N}^{+}(\alpha, \beta, \theta)^{\frac{1}{2}}\right)$ as $\alpha \rightarrow 0$. Similarly, if for all $0<\beta<1$

$$
\text { (1.1.4) } \quad \lim _{\alpha \rightarrow 0} \sup _{\alpha} \frac{N_{\phi}(\alpha, \beta, \theta)-N^{+}(\alpha, \beta, \theta)}{\log N^{+}(\alpha, \beta, \theta)} \leq A(\beta, \theta)
$$

we shall say that $\left\{\phi_{n}^{\gamma} ; \gamma \in \Gamma\right\}$ is deficient in the sense of Bahadur at $\theta$ of order $O\left(\log \mathrm{N}^{+}(\alpha, \beta, \theta)\right)$ as $\alpha \rightarrow 0$.

This way of introducing Bahadur efficiency differs from the original definition in BAHADUR (1960). He introduced the concept of the slope of a sequence of tests: For each $n=1,2, \ldots$ let $T_{n}(s)$ be an extended real-valued function such that $T_{n}$ is $A$-measurable and depends on $s$ only through $\left(x_{1}, \ldots, x_{n}\right) ; T_{n}$ is to be thought of as a test statistic, large values of $T_{n}$ being significant. Let $I_{n}(t)=\sup \left\{P_{\theta}\left(T_{n} \geq t\right) ; \theta \in \Theta_{0}\right\}$ denote the tail probability (level attained at $T_{n}=t$ ). The sequence $\left\{T_{n}\right\}$ has exact slope $c(\theta)$ when $\theta$ obtains if $-n^{-1} \log 1_{n}\left(T_{n}\right) \rightarrow \frac{1}{2} c(\theta)$ in $P_{\theta}$-probability. If the sequence $\left\{T_{n}^{*}\right\}$ has exact slope $c^{*}(\theta)$, the Bahadur efficiency of $\left\{T_{n}\right\}$ with respect to $\left\{T_{n}^{*}\right\}$ equals $c(\theta) / c^{*}(\theta)$ (cf. BAHADUR (1960)).

In [19] RAGHAVACHARI proves that for each $\theta \in \theta-\theta_{0}$

(1.1.5) $\quad \lim _{n \rightarrow \infty} \sup ^{-1} \log l_{n}\left(T_{n}\right) \leq I\left(\theta, \theta_{0}\right)$

with probability one when $\theta$ obtains, where

$(1.1 .6)$

$$
I\left(\theta, \theta_{0}\right)=\inf \left\{I\left(\theta_{,} \theta_{0}\right) ; \theta_{0} \in \theta_{0}\right\}
$$

and

(1.1.7) $I\left(\theta, \theta_{0}\right)=\int \log \left\{f(x ; \theta) / f\left(x ; \theta_{0}\right)\right\} d P_{\theta}(x)$.

Thus the maximal slope of a family of tests is $2 I\left(\theta, \theta_{0}\right)$. The number $I\left(\theta, \theta_{0}\right)$ is called the Kullback-Leibler information number.

In this framework the following theorem of Bahadur is of particular interest.

THEOREM 1.1.1 (BAHADUR (1965)). Let $1_{n}\left(T_{n}^{L R}\right)$ denote the tail probability of the LR test. Under the (rather strong) assumptions $1, \ldots, 6$ in [2] it holds that for each $\theta \in \theta-\theta_{0}$

(1.1.8) $\quad \lim _{n \rightarrow \infty}-n^{-1} \log 1_{n}\left(T_{n}^{L R}\right)=I\left(\theta, \Theta_{0}\right)$

with probability one when $\theta$ obtains.

In BAHADUR and RAGHAVACHARI (1970) this result is extended to more 
general cases; they state two conditions under which tests are asymptotically optimal in the sense of exact slopes.

In this "non-local" or "fixed alternative" method of comparison of tests one considers in fact the rate of exponential convergence of the size of a test to zero. This concept is also the subject of a paper of BROWN (1971). He extends the parameter space to obtain a good structure by adding densities of the form

$$
c\left(\xi, \theta_{0}, \theta_{1}\right)\left\{p_{\theta_{0}}(x)\right\}^{1-\xi}\left\{p_{\theta_{1}}(x)\right\}^{\xi}, \quad \theta_{0} \in \theta_{0}, \theta_{1} \in \theta_{1}, 0<\xi<1,
$$

where $c$ is a normalizing constant. Let $\lambda_{n}^{*}$ be the LR statistic for the "larger" problem of testing $\theta_{0}^{*}$ against $\theta_{1}^{*}$, where $\theta_{0} \subset \theta_{0}^{*}$ and $\theta_{1} \subset \theta_{1}^{*}$. Usually $\theta_{0}=\theta_{0}^{*}$, often however $\theta_{1} \neq \theta_{1}^{*}$ so that $\lambda_{n}^{*}$ is essentially different from the LR statistic for the original problem. Then he showed that $\lambda_{\mathrm{n}}^{*}$ is asymptotically optimal in the following sense. Let $\alpha_{n}^{T}$ and $\beta_{n}^{T}(\theta)$ be the significance level and power (in $\theta$ ) of a sequence of tests $\left\{T_{n}\right\}$ of $\theta_{0}$ against $\theta_{1}$; let $\alpha_{n}^{*}$ and $\beta_{n}^{*}(\theta)$ be the significance level and power (in $\theta$ ) of the test which rejects if $\lambda_{n}^{*}>c_{n}$. Then under appropriate regularity conditions the following result is valid.

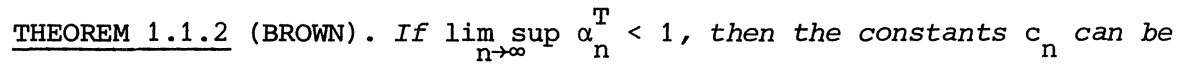
chosen so that

$$
\alpha_{n}^{*} \leq \alpha_{n}^{T}
$$

and for all $\theta \in \Theta_{1}$

$$
\underset{n \rightarrow \infty}{\lim \inf }\left\{n^{-1} \log \left(1-\beta_{n}^{T}(\theta)\right)-n^{-1} \log \left(1-\beta_{n}^{*}(\theta)\right)\right\} \geq 0
$$

A quite different optimality property has been obtained by BOROVKOV (1975), who showed that for a broad class of testing problems Bayes tests with respect to smooth a priori distributions are asymptotic equivalent to the IR test.

\subsection{LR TESTS IN EXPONENTIAL FAMILIES}

In section 1.1 properties of LR tests have been described for fairly general families of distributions. In the remainder of this study we restrict attention to families of distributions with a special structure: exponential families. 
A $\mathrm{k}$-dimensional random variable $\mathrm{X}$ is distributed according to a $k$-parameter exponential family if the density of $x$ with respect to a $\sigma$-finite non-degenerate measure $\mu$ is of the form

$$
\text { (1.2.1) } \quad \mathrm{dP}_{\theta}(\mathrm{x})=\exp \left\{\theta^{\prime} \mathrm{x}-\psi(\theta)\right\} \mathrm{d} \mu(\mathrm{x}), \quad \theta \in \theta \subset \mathbb{R}^{\mathrm{k}}, \mathrm{x} \in \mathbb{R}^{\mathrm{k}} \text {, }
$$

where $k \in \mathbb{N}$,

$$
\text { (1.2.2) } \theta=\left\{\theta ; \int \exp \left\{\theta^{\prime} \mathrm{x}\right\} \mathrm{d} \mu(\mathrm{x})<\infty\right\}
$$

and

$$
\text { (1.2.3) } \psi(\theta)=\log \int \exp \left\{\theta^{\prime} \mathrm{x}\right\} \mathrm{d} \mu(\mathrm{x}), \quad \theta \in \theta .
$$

Here $\theta^{\prime} x$ denotes the inner product of $\theta$ and $x$. The space $\theta$ is called the natural parameter space. It is well known that $\theta$ is a convex set in $\mathbb{R}^{k}$ and we will assume that it contains an open set in that space. (Otherwise it is always possible to reparameterize to a lower dimensional exponential family where the condition does hold.) Moreover, we may write

$$
\mathrm{dP}_{\theta}(\mathrm{x})=\exp \left\{\left(\theta-\theta_{0}\right) \cdot \mathrm{x}-\psi(\theta)+\psi\left(\theta_{0}\right)\right\} \mathrm{dP}_{\theta_{0}}(\mathrm{x})
$$

Letting $\tilde{\theta}=\theta-\theta_{0}$ we see that we can take our special point $\theta_{0}$ to be the origin without loss of generality, in which case $\mathrm{P}_{\theta_{0}}$ plays the role of the measure $\mu$. Hence assume

$$
\text { (1.2.4) int } \theta \neq \varnothing \text { and } 0 \in \text { int } \theta \text {. }
$$

Thus $\mu$ is a non-degenerate probability measure and $\psi$ is the log moment generating function of $\mu$.

In many books exponential families are defined by densities of the form

$$
\operatorname{dP}_{\theta}^{X}(x)=C(\theta) \exp \left\{\sum_{j=1}^{k} Q_{j}(\theta) T_{j}(x)\right\} h(x) d \nu(x) .
$$

Since the distribution $\mathrm{P}_{\theta}^{\mathrm{T}}$ of the sufficient vector

$$
(1.2 .5) . \quad T=\left(T_{1}(X), \ldots, T_{k}(X)\right)
$$

is of the form

$$
\mathrm{dP}_{\theta}^{T}(t)=c(\theta) \exp \left\{\sum_{j=1}^{k} Q_{j}(\theta) t_{j}\right\} d \mu(t)
$$


and we only consider procedures based on the sufficient statistic, it is justified to describe exponential families by (1.2.1), where a more natural parameterization is employed.

Let $\theta^{*}=\left\{\theta \in \Theta ; E_{\theta}\|x\|<\infty\right\}$, where $\|\cdot\|$ denotes the Euclidean norm. For $\theta \in \Theta^{*}$ we define

$$
(1.2 .6) \quad \lambda(\theta)=E_{\theta} X
$$

The mapping $\lambda$ is $1-1$ on $\theta^{*}$ (cf. lemma 2.2 in [5]). Defining

$$
\Lambda=\lambda\left(\theta^{*}\right)=\left\{\lambda(\theta) ; \theta \in \theta^{*}\right\}
$$

the inverse mapping, denoted by $\lambda^{-1}$, is defined on $\Lambda$. For $\theta \in$ int $\theta$ the vector of expectations and the covariance matrix of $\mathrm{P}_{\theta}$ are given by $\lambda(\theta)=\operatorname{grad} \psi(\theta)$ and $\Sigma_{\theta}$, the matrix of second order partial derivatives, respectively. Note that $\psi$ is a convex function on $\theta$. In the one-parameter case the variance is denoted by $\sigma^{2}(\theta)$. It turns out that the KullbackLeibler information number is given by

$(1.2 .7) \quad I\left(\theta, \theta_{0}\right)=\psi\left(\theta_{0}\right)-\psi(\theta)+\left(\theta-\theta_{0}\right) \cdot \lambda(\theta)$

(cf. (1.1.7)) for $\theta_{0} \in \theta, \theta \in \theta^{*}$. The function I can be related to the (Euclidean) distance between $\theta$ and $\theta_{0}$ (cf. lemma 2.2.2, lemma 3.2.2 and lemma 4.1.2); therefore we sometimes refer to $I\left(\theta_{,} \theta_{0}\right)$ as "the KullbackLeibler distance" from $\mathrm{P}_{\theta}$ to $\mathrm{P}_{\theta_{0}}$.

EXAMPLE 1.2.1. Let $\mu$ be the probability measure corresponding to the standard normal distribution, then $\mathrm{P}_{\theta}$ corresponds to the normal $\mathrm{N}(\theta, 1)$ distribution, $\psi(\theta)=\frac{1}{2} \theta^{2}$ and $I\left(\theta, \theta_{0}\right)=\frac{1}{2}\left(\theta-\theta_{0}\right)^{2}$.

EXAMPLE 1.2.2. Let $\mathrm{x}$ be normally $\mathrm{N}\left(\xi, \sigma^{2}\right)$ distributed, then $\left(\mathrm{x}, \mathrm{x}^{2}\right)$ is the (sufficient) statistic $T$ appearing in (1.2.5). Let $\mu$ correspond to the distribution of $\left(\mathrm{x}, \mathrm{x}^{2}\right)$ under $\xi=0$ and $\sigma^{2}=1$ then $\theta=\left(\theta^{(1)}, \theta^{(2)}\right)=$ $\left(\xi \sigma^{-2}, \frac{1}{2}-\frac{1}{2} \sigma^{-2}\right)$ and $\psi(\theta)=\frac{1}{4}\left(\theta^{(1)}\right)^{2}\left(\frac{1}{2}-\theta^{(2)}\right)^{-1}-\frac{1}{2} \log \left(1-2 \theta^{(2)}\right)$.

Since $I\left(\theta, \theta_{0}\right) \geq 0$ and thus $\theta^{\prime} \lambda(\theta)-\psi(\theta) \geq \theta_{0}^{\prime} \lambda(\theta)-\psi\left(\theta_{0}\right)$ for all $\theta_{0} \in \theta, \theta \in \theta^{*}$, it follows that

$$
\text { (1.2.8) } \quad \lambda^{-1}(\mathrm{x})^{\prime} \mathrm{x}-\psi\left(\lambda^{-1}(\mathrm{x})\right)=\sup _{\theta \in \Theta}\left\{\theta^{\prime} \mathrm{x}-\psi(\theta)\right\}
$$

for all $\mathrm{x} \in \Lambda$. 
Let $\mathrm{x}_{1}, \mathrm{x}_{2}, \ldots, \mathrm{x}_{\mathrm{n}}$ be i.i.d. random variables with distribution $P_{\theta}$ given by $(1.2 .1)$ and let

$$
\text { (1.2.9) } \quad \bar{x}_{n}=n^{-1} \sum_{i=1}^{n} x_{i}, \quad n=1,2, \ldots \text {. }
$$

The distribution of $\bar{x}_{n}$ will be denoted by $\bar{P}_{\theta}^{n}$; if $\theta=0$ we often write $\bar{\mu}^{-n}$. In the one-parameter case $\tilde{P}_{\theta}^{n}$ denotes the distribution of $n^{\frac{1}{2}}\left\{\bar{x}_{n}-\lambda(\theta)\right\} \sigma(\theta)^{-1}$. Note that $\lambda^{-1}\left(\bar{x}_{n}\right)$ is the maximum likelihood estimator of $\theta$ if $\bar{x}_{n} \in \Lambda$.

In the sequel we consider the following testing problem:

$$
\mathrm{H}_{0}: \theta \in \theta_{0}
$$

is tested against

$$
\mathrm{H}_{1}: \theta \in \Theta_{1}
$$

at level $\alpha_{n}$, where $\theta_{0}$ is a subset of $\theta$ and (except for section 3.8) $\theta_{1}=\theta-\theta_{0}$. Note that the level of significance is not fixed but depends, in general, on the number of observations.

Let $s=\left(x_{1}, x_{2}, \ldots\right)$, define $\bar{x}_{n}=n^{-1} \sum_{i=1}^{n} x_{i}, n=1,2, \ldots$, then $T_{n}^{L R}(s)=\lambda^{-1}\left(\bar{x}_{n}\right) \cdot \bar{x}_{n}-\psi\left(\lambda^{-1}\left(\bar{x}_{n}\right)\right)-\sup _{\theta_{0} \in \theta_{0}}\left\{\theta_{0} \bar{x}_{n}-\psi\left(\theta_{0}\right)\right\}=I\left(\lambda^{-1}\left(\bar{x}_{n}\right), \theta_{0}\right)$ for all $\bar{x}_{\mathrm{n}} \in \Lambda$. Hence, if $\bar{x}_{\mathrm{n}} \in \Lambda$,

$$
\phi_{n}^{L R}(s)= \begin{cases}1 & > \\ \delta_{n} \text { if } I\left(\lambda^{-1}\left(\bar{x}_{n}\right), \theta_{0}\right) & =d_{n} . \\ 0 & <\end{cases}
$$

Since $\phi_{n}^{L R}$ is a function of $\bar{x}_{n}$ only we often write $\phi_{n}^{L R}\left(\bar{x}_{n}\right)$ in lieu of $\phi_{n}^{L R}(S)$. In this notation the mapping $\phi_{n}^{L R}: \mathbb{R}^{k} \rightarrow[0,1]$ is defined by

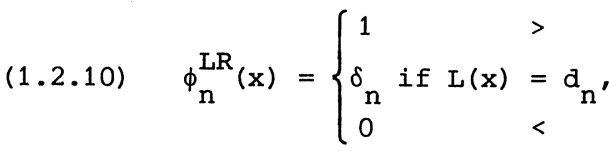
where $L(x)=\sup _{\theta \in \theta^{\prime}}\left\{\theta^{\prime} x-\psi(\theta)\right\}-\sup _{\theta_{0} \in \Theta_{0}}\left\{\theta_{0}^{\prime} x-\psi\left(\theta_{0}\right)\right\}$. We shall use this definition in the sequel.

For one particular exponential family, the multinomial distribution, optimality of the LR test has been studied by HOEFFDING (1965a). In this paper the following proposition is made precise. "If a given test of size $\alpha_{n}$ is 'sufficiently different' from a LR test, then there is a LR test of size $\leq \alpha_{n}$ which is considerably more powerful than the given test at 'most' 
parameter points in the set of alternatives when $\mathrm{n}$ is large enough, provided that $\alpha_{n} \rightarrow 0$ at a suitable rate". Here "considerably more powerful" is to be interpreted in the sense that the ratio of the error probabilities of the second kind of the two tests tends to zero more rapidly than any power of $n$. HERR (1967) (partially) extends Hoeffding's result to non-singular multivariate normal distributions.

If the LR test is much better than a given test for most alternatives, it is natural to ask how much worse it can be for the remaining alternatives or sequences of alternatives. To measure this it is useful to consider the shortcoming of the LR test. Let $\Phi_{n}\left(\alpha_{n}\right)$ be the class of all level- $\alpha_{n}$ tests $\phi$ of $\mathrm{H}_{0}$ against $\mathrm{H}_{1}$ and let $\beta_{\mathrm{n}}^{\phi}(\theta)$ be the power of a particular test $\phi$ at $\theta$ all based on $n$ observations, then the envelope power function is defined by

$$
\beta_{n}^{+}(\theta)=\sup _{\phi \in \Phi_{n}}\left(\alpha_{n}\right) \beta_{n}^{\phi}(\theta) .
$$

Denoting the power of the LR test at $\theta$ by $\beta_{n}^{L R}(\theta)$, the shortcoming of the size- $\alpha_{n}$ LR test for a given $n$ is defined by

$$
R_{n}(\theta)=\beta_{n}^{+}(\theta)-\beta_{n}^{L R}(\theta), \quad \theta \in \theta_{1} \text {. }
$$

OOSTERHOFF and VAN ZWET (1970) investigated the behaviour of $R_{n}$ in the multinomial case mainly for testing problems of a simple hypothesis against a composite alternative. They proved that under a condition on the exponen: tial rate of convergence to zero of $\alpha_{n}$ as $n \rightarrow \infty, R_{n}$ converges uniformly to zero. Hence the LR test is an asymptotically optimal test in the sense of shortcoming.

This criterion of optimality seems to be stronger than Wald's asymptotic most stringency. However, since wald considers testing problems with $\alpha_{n}=\alpha$ is fixed, direct comparison is impossible. Since as a rule the LR test does not have vanishing shortcoming for fixed $\alpha$ the optimality of the LR test seems to be stronger for testing problems where $\alpha_{n} \rightarrow 0$ as $n \rightarrow \infty$. On the other hand the concept of (uniformly) vanishing shortcoming supplements Bahadur's and Brown's approach. The approach of Bahadur is rather unbalanced since probabilities of errors of the second kind are kept fixed and only the probability of an error of the first kind is sent to zero. Moreover, in typical cases two sequences of size- $\alpha_{n}$ tests with fixed powers $\beta_{0}$ and $\beta_{1}$ at $\theta_{1} \quad\left(0<\beta_{0} \neq \beta_{1}<1\right)$, respectively, have the same exact slope and hence the Bahadur efficiency concept does not discriminate between these two sequences of tests. The same lack of sensitivity with respect to fixed 
power differences is a weak feature of Brown's criterion too. In a way uniformly vanishing shortcoming can be regarded as an intermediate between optimality in the sense of a "fixed alternative" and a "contiguous alternative" approach.

The optimality of LR tests in the sense of shortcoming is related to testing problems with levels of significance $\alpha_{n}$ tending to zero as the following example shows.

EXAMPLE 1.2 .3 . Let $\mathrm{x}_{1}, \mathrm{x}_{2}, \ldots$ be i.i.d. random variables with a normal $N(\theta, 1)$ distribution. The hypothesis $\mathrm{H}_{0}: \theta=0$ is tested against $\mathrm{H}_{1}: \theta \neq 0$ with level of significance $\alpha=0,05$. Then $\beta_{n}^{+}\left(n^{-\frac{1}{2}}\right)=P_{n^{-\frac{1}{2}}}\left(\bar{x}_{n} n^{\frac{1}{2}} \geq u_{0,95}\right)=$ 0,26 and $\beta_{n}^{L R}\left(n^{-\frac{1}{2}}\right)=P_{n^{-\frac{1}{2}}}\left(\left|\bar{x}_{n} n^{\frac{1}{2}}\right| \geq u_{0,975}\right)=0,17$ where $u_{t}$ is defined by $P_{0}\left(x_{1} \leq u_{t}\right)=t(0<t<1)$. Hence $\sup _{\theta \in \Theta_{1}} R_{n}(\theta) \geq 0,09$ for all $n$, implying that $\sup _{\theta \in \Theta_{1}} R_{n}(\theta)$ does not converge to zero.

In chapter II and III the results of Oosterhoff and Van zwet will be extended to more general cases. In chapter II the one-parameter exponential families are treated. It turns out that the shortcoming of the LR test tends to zero both pointwise and uniformly on the intersection of $\theta_{1}$ with a compact subset of int $\Theta$. Under some condition on the LR test uniformly vanishing shortcoming over $\theta_{1}$ is established. As a consequence it can be proved that $\lim _{n \rightarrow \infty} \sup _{\theta \in \Theta_{1}} R_{n}(\theta)=0$ if $\theta_{0}$ is contained in a compact subset of int $\theta$ and a condition is imposed on the rate of convergence of $\alpha_{n}$. The results for one-parameter exponential families are more explicit and slightly stronger than for $k$-parameter exponential families $(k \geq 2)$ as is shown by the examples in section 3.1. This explains the separate treatment of the one-parameter case. The third chapter is devoted to generalizations of the shortcoming results obtained in chapter II to $k$-parameter exponential families. Large deviation theory plays an important role in this chapter. A result of HOEFFDING (1965b) for the multinomial distribution, partially generalized by EFRON and TRUAX (1968), is extended to k-parameter exponential families.

Chapter IV is devoted to the relation between vanishing shortcoming and Bahadur deficiency (cf. section 1.1). It turns out that vanishing shortcoming is equivalent to Bahadur deficiency of order $O\left(N^{+}(\alpha, \beta, \theta)^{\frac{1}{2}}\right)$ as $\alpha \rightarrow 0$.

In chapter $\mathrm{V}$ the Bahadur deficiency of the LR test is investigated. In typical cases the Bahadur deficiency of the LR test is of order 
$O\left(\log \mathrm{N}^{+}(\alpha, \beta, \theta)\right)$ as $\alpha \rightarrow 0$. As far as we know this is the first investigation of Bahadur deficiency of families of tests. 

CHAPTER II

THE ONE-PARAMETER CASE

\subsection{INTRODUCTION}

Let $x_{1}, x_{2}, \ldots, x_{n}$ be i.i.d. real valued random variables $(n=1,2, \ldots)$, distributed according to a one-parameter exponential family: $\left\{\mathrm{P}_{\theta} ; \theta \in \Theta\right\}$. Such a family will be represented in its standard form by

- (2.1.1) $\quad \mathrm{dP}_{\theta}(\mathrm{x})=\exp \{\theta \mathrm{x}-\psi(\theta)\} \mathrm{d} \mu(\mathrm{x}), \quad$ for all $\mathrm{x} \in \mathbb{R}$.

Here $\mu$ is a non-degenerate probability measure and $\psi(\theta)$ is the log moment generating function of $\mu$. We assume that int $\theta \neq \varnothing$ and $0 \in$ int $\theta$ (cf. (1.2.4)). The natural parameter space $\theta$ is a (possible infinite) interval.

We consider the following testing problem. For each $n \in \mathbb{N}$ the hypothesis

$$
\mathrm{H}_{0}: \theta \in \Theta_{0}
$$

is tested against

$$
\mathrm{H}_{1}: \theta \in \theta_{1}=\theta-\theta_{0}
$$

at level $\alpha_{n}$ with the available observations $x_{1}, \ldots, x_{n}$, where $\lim _{n \rightarrow \infty} \alpha_{n}=0$.

Let $\phi_{n}^{\operatorname{LR}}\left(n^{-1} \sum_{i=1}^{n} x_{i}\right)$ denote the critical function of the size- $\alpha_{n} L R$ test of $\mathrm{H}_{0}$ against $\mathrm{H}_{1}$ based on $\mathrm{x}_{1}, \ldots, \mathrm{x}_{\mathrm{n}}$ and let $\beta_{n}^{L R}$ be its power function. We investigate the behaviour of $R_{n}(\theta)$ as $n \rightarrow \infty$, where $R_{n}$ denotes the shortcoming of the size- $\alpha_{n}$ LR test.

In fact we shall prove that $\lim _{n \rightarrow \infty} R_{n}(\theta)=0$ uniformly on $\theta_{1}$ in three different cases:

A. $\Theta_{0}$ is contained in a compact subset of int $\theta$ and a condition is imposed on the rate of convergence of $\alpha_{n}$.

B. $\theta_{1}$ is contained in a compact subset of int $\theta$.

C. Some conditions are imposed on the second and third central moment of $x$. These results are corollaries of theorem 2.5.1. 
Moreover we shall prove that $\lim _{n \rightarrow \infty} R_{n}(\theta)=0$ uniformly on $K \wedge \theta_{1}$ for each compact subset $\mathrm{K}$ of int $\theta$ (theorem 2.7.1). Note that case $B$ is a particular case of theorem 2.7.1.

Obviously theorem 2.7.1 implies the weaker result $\lim _{n \rightarrow \infty} R_{n}(\theta)=0$ pointwise for each $\theta \in$ int $\theta$. It can also be shown that $\lim _{n \rightarrow \infty} R_{n}(\theta)=0$ for boundary points of $\theta$ in $\theta_{1}$.

\subsection{PRELIMINARIES}

Before proving the results mentioned above we derive some properties of the functions $\lambda(\theta)$ and $\psi(\theta)$, the Kullback-Leibler information $I(n, \theta)$ and the LR test.

The following notation will be used throughout this chapter:

$$
\bar{\theta}=\sup \theta \text { and } \underline{\theta}=\inf \theta \text {, }
$$

where $\bar{\theta}=\infty$ if $\theta$ is not bounded above, and $\underline{\theta}=-\infty$ if $\theta$ is not bounded below. Note that $\underline{\theta}<0<\bar{\theta}$. Similarly we define

$$
\bar{\theta}_{i}=\sup \theta_{i} \quad \text { and } \quad \underline{\theta}_{i}=\inf \theta_{i} \quad(i=0,1) .
$$

Furthermore $\lambda(\theta)=E_{\theta} X=\int x \exp \{\theta x-\psi(\theta)\} d \mu(x)$ is defined for all $\theta \epsilon \theta$, since for $\theta>0|x \exp (\theta x)| \leq|x|$ on $(-\infty, 0]$, for $\theta<0|x \exp (\theta x)| \leq|x|$ on $[0, \infty)$ and $\int|x| d \mu(x)<\infty$ (if $\bar{\theta} \in \theta$ we may have $\lambda(\bar{\theta})=\infty$ and if $\underline{\theta} \epsilon \theta$ we may have $\lambda(\underline{\theta})=-\infty)$.

Again writing

$$
\theta^{*}=\left\{\theta \in \theta_{i}|\lambda(\theta)|<\infty\right\} \text { and } \Lambda=\lambda\left(\theta^{*}\right) \text {, }
$$

let

$$
\bar{\lambda}=\sup \Lambda \text { and } \underline{\lambda}=\inf \Lambda \text {. }
$$

Some properties of the functions $\psi$ and $\lambda$ are stated in the following

LEMMA 2.2.1. The functions $\psi$ and $\lambda$ are continuous on int $\theta$. Moreover, if $\bar{\theta} \in \theta$ then $\lim _{\theta \uparrow \bar{\theta}} \psi(\theta)=\psi(\bar{\theta})$ and $\lim _{\theta \uparrow \bar{\theta}} \lambda(\theta)=\lambda(\bar{\theta})$; if $\underline{\theta} \epsilon$ then $\lim _{\theta \downarrow \underline{\theta}} \psi(\theta)=\psi(\underline{\theta})$ and $\lim _{\theta \downarrow \underline{\theta}} \lambda(\theta)=\lambda(\underline{\theta})$.

PROOF. For the first statement see LEHMANN (1959) section 2.7. Suppose $\bar{\theta} \in \theta$. Let $\theta \uparrow \bar{\theta} ;$ since $\bar{\theta}>0$ we may assume $\theta>0$. Consider the inequality (2.2.1) $\exp (\theta x) \leq 1+\exp (\bar{\theta} \mathbf{x}) \quad$ for all $\mathrm{x} \in \mathbb{R}$. 
The function on the right of (2.2.1) is integrable, and hence by the dominated convergence theorem $\lim _{\theta \uparrow \bar{\theta}} \int \exp (\theta x) d \mu(x)=\int \lim _{\theta \uparrow \bar{\theta}} \exp (\theta x) d \mu(x)=$ $\int \exp (\bar{\theta} x) d \mu(x)$, implying $\lim _{\theta \uparrow \bar{\theta}} \psi(\theta)=\psi(\bar{\theta})$.

For the function $\lambda$ we have $\lambda(\theta)=\exp (-\psi(\theta)) \int \mathbf{x} \exp (\theta \mathbf{x}) d \mu(\mathbf{x})$. Splitting the region of integration $\mathbb{R}$ into $(-\infty, 0]$ and $(0, \infty)$, and applying the dominated convergence theorem and the monotone convergence theorem one obtains $\lim _{\theta \uparrow \bar{\theta}} \int \mathrm{x} \exp (\theta x) d \mu(x)=\int x \exp (\bar{\theta} x) d \mu(x)$. In combination with $\lim _{\theta \uparrow \bar{\theta}}$ $\exp (-\psi(\theta))=\exp (-\psi(\bar{\theta}))$ this completes the proof of $\lim _{\theta \uparrow \bar{\theta}} \lambda(\theta)=\lambda(\bar{\theta})$. The proof of the statements about $\underline{\theta}$ is similar.

As a corollary we have that $\Lambda$ is an interval of the real line. In chapter I we have already introduced the Kullback-Leibler information number (cf. (1.1.7) and (1.2.7))

$$
I(n, \theta)=E_{\eta} \log \mathrm{dP}_{\eta} / \mathrm{dP}_{\theta}(\mathrm{x})=\psi(\theta)-\psi(\eta)+(n-\theta) \lambda(\eta) .
$$

In the next lemma some further properties of $I(n, \theta)$ are listed.

LEMMA 2.2.2.

(i) I $(n, \theta)$ is a strictly convex function of $\theta$ on $\theta$ for any $n \in \theta^{*}$.

(ii) $I(n, \theta)$ is a strictly decreasing-increasing continuous function of $\eta$ on $\theta^{*}$ with minimum 0 in $\eta=\theta$ and $\lim _{n \rightarrow \theta} I(n, \theta)=0$ for all $\theta \in \theta$.

(iii) For any $\eta, \theta \in$ int $\theta$

$$
(2.2 .3) \quad I(n, \theta)=\frac{1}{2}(n-\theta)^{2} \sigma^{2}(\xi)
$$

with $\xi$ between $\eta$ and $\theta$.

(iv) For any $\eta, \theta, \xi \in \theta$ with finite $\lambda(\eta)$ and $\lambda(\xi)$

$$
I(n, \theta)-I(\xi, \theta)=(\xi-\theta)(\lambda(n)-\lambda(\xi))+I(n, \xi)
$$

PROOF. Assertions (i) and (ii) follow by differentiation of (2.2.2) on int $\theta$, application of lemma 2.2 .1 and $\lim _{n \rightarrow \theta} \lambda(n)(n-\theta)=0$ by dominated convergence for boundary points $\theta$. Assertion (iii) is an application of the mean value theorem, and (iv) is obtained by substitution of (2.2.2):

$$
\begin{aligned}
I(n, \theta)-I(\xi, \theta) & =\psi(\theta)-\psi(n)+(n-\theta) \lambda(n)-\psi(\theta)+\psi(\xi)-(\xi-\theta) \lambda(\xi) \\
& =(\xi-\theta)(\lambda(n)-\lambda(\xi))+\psi(\xi)-\psi(n)+(n-\xi) \lambda(n) \\
& =(\xi-\theta)(\lambda(n)-\lambda(\xi))+I(n, \xi) \cdot
\end{aligned}
$$


EXAMPLE 2.2.1. Let $\mu$ be the standard normal distribution. Then we have $\psi(\theta)=\frac{1}{2} \theta^{2}, \lambda(\theta)=\theta$ and $I(n, \theta)=\frac{1}{2}(n-\theta)^{2}$.

EXAMPLE 2.2.2. Let $\mu$ be absolutely continuous with respect to Lebesgue measure on $\mathbb{R}$ with density $\exp (-\mathbf{x}), 0<\mathbf{x}<\infty$. We have $\psi(\theta)=-\log (1-\theta)$, $\lambda(\theta)=(1-\theta)^{-1}$ and $I(\eta, \theta)=\log \left\{(1-\eta)(1-\theta)^{-1}\right\}+(n-\theta)(1-\eta)^{-1}$.

EXAMPLE 2.2.3. Let $\mu$ be absolutely continuous with respect to Lebesgue measure on $\mathbb{R}$ with density $c x^{-2} \exp (-x), 1<\mathrm{x}<\infty$, where $c=$ $\left\{\int_{1}^{\infty} x^{-2} \exp (-x) d x\right\}^{-1}$. Then we have $\theta=(-\infty, 1]$ and since $\lambda(1)=\infty$ obviously $I(1, \theta)=\infty$ for all $\theta<1$.

Now consider the LR test a little more closely. The critical function of the size- $\alpha_{n}$ LR test of $H_{0}$ against $H_{1}$ is defined by

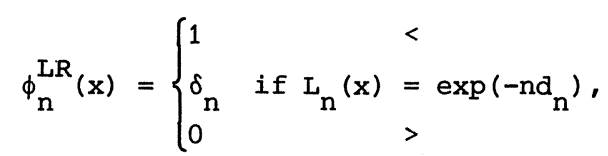

where

$$
L_{n}(x)=\frac{\sup _{\theta_{0} \in \theta_{0}} \exp \left\{n \theta_{0} x-n \psi\left(\theta_{0}\right)\right\}}{\sup _{\theta \epsilon \Theta} \exp \{n \theta x-n \psi(\theta)\}},
$$

and where $0 \leq \delta_{n} \leq 1$ and $d_{n} \geq 0$ are determined by

$$
\sup _{\theta_{0} \in \Theta_{0}} E_{\theta_{0}} \phi_{n}^{L R}\left(\bar{x}_{n}\right)=\alpha_{n} \quad(n=1,2, \ldots) \text {. }
$$

In the particular case that $x \in \Lambda(2.2 .5)$ reduces to

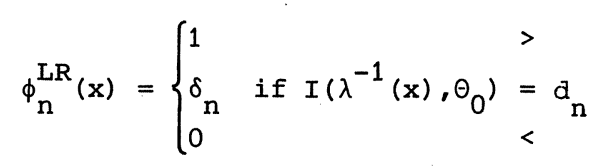

$(\mathrm{n}=1,2, \ldots)(\mathrm{cf} .(1.2 .9)$ and $(1.2 .7))$.

2.3. RELATION BETWEEN $\alpha_{n}$ AND $d_{n}$.

In this section we state and prove an inequality between $\alpha_{n}$ and $d_{n}$.

LEMMA 2.3.1. Let $\mathrm{x}_{1}, \ldots, \mathrm{x}_{\mathrm{n}}$ be a random sample from a one-parameter exponential family $(n=1,2, \ldots)$. Consider the testing problem $\mathrm{H}_{0}: \theta \in \theta_{0}$ against $\mathrm{H}_{1}: \theta \in \theta_{1}=\theta-\theta_{0}$ at level $\alpha_{\mathrm{n}}$ with the available observations $\mathrm{x}_{1}, \ldots, \mathrm{x}_{\mathrm{n}}$. 
Let $\phi_{\mathrm{n}}^{\mathrm{LR}}$ be the critical function of the size- $\alpha_{\mathrm{n}}$ LR test as defined in (2.2.5). Then the following inequality holds:

(2.3.1) $\quad \alpha_{n} \leq 5 \exp \left(-n d_{n}\right)$.

REMARK. There is no restriction on $\mathrm{H}_{0}, \mathrm{H}_{1}$ or $\alpha_{n}$ in the lemma. Under some conditions on $\mathrm{H}_{0}, \mathrm{H}_{1}$ or $\alpha_{n}$ (for example if $\theta_{0}$ is a compact subset of int $\theta$ ) one can prove that $\alpha_{n}=o(1) \exp \left(-n d_{n}\right)$ as $n \rightarrow \infty$. In section 2.6 such results will be derived. The constant 5 in this inequality can be improved to 2 , which is the sharpenest constant as the following example shows.

EXAMPLE 2.3.1. Let $\mathrm{x}_{1}, \ldots, \mathrm{x}_{\mathrm{n}}$ be $\mathrm{n}$ independent Bernoulli random variables. In terms of exponential families: the underlying distribution $\mu$ is given by $\mu(0)=\mu(1)=\frac{1}{2}$.

Take $\mathrm{H}_{0}: \theta=0$ (corresponding to probability of success $\frac{1}{2}$ ) and $\mathrm{H}_{1}: \theta \neq 0$. We have $I(\theta, 0)=-\log \left(1+e^{\theta}\right)+\log 2+\left(1+e^{\theta}\right)^{-1} \theta e^{\theta}$ and it is easy to see that $0 \leq I(\theta, 0) \leq \log 2$. Choosing $\alpha_{n}=2\left(\frac{1}{2}\right)^{n}$ the LR test is nonrandomized and $d_{n}=\log 2$; so we have $\alpha_{n}=2 \exp \left(-n d_{n}\right)$.

PROOF OF LEMMA 2.3.1. Let $\mathrm{n}$ be fixed. Assume $d_{n}>0$ (otherwise the lemma is trivial). We consider two cases a) and b).

a) There exists $\theta_{0 \mathrm{n}} \in \theta_{0}$ such that

$$
P_{\theta_{0 n}}\left(\phi_{n}^{L R}\left(\bar{x}_{n}\right)>0, \bar{x}_{n} \in \Lambda\right) \geq \frac{1}{2} \alpha_{n} \text {. }
$$

It follows from $(2.2 .6)$ that in this case

$$
P_{\theta_{0 n}}\left(I\left(\lambda^{-1}\left(\bar{x}_{n}\right), \theta_{0}\right) \geq d_{n}, \bar{x}_{n} \in \Lambda\right) \geq \frac{1}{2} \alpha_{n},
$$

and hence a fortiori

$$
\text { (2.3.2) } \quad P_{\theta_{0 n}}\left(I\left(\lambda^{-1}\left(\bar{x}_{n}\right), \theta_{O n}\right) \geq d_{n}, \bar{x}_{n} \in \Lambda\right) \geq \frac{1}{2}_{2}{ }_{n} .
$$

Define points $\theta_{O_{n}}^{\prime}$ and $\theta_{O_{n}^{\prime \prime}}$ in $\theta$ by the conditions $I\left(\theta_{O_{n}}^{\prime}, \theta_{0 n}\right)=$ $I\left(\theta_{O n}^{\prime \prime}, \theta_{O n}\right)=d_{n}$ and $\theta_{O n}^{\prime}<\theta_{O n}<\theta_{O n}^{\prime \prime}$. From (2.3.2) and the continuity of $I\left(\cdot, \theta_{0 n}\right)$ it is seen that at least one of the points $\theta_{0 n}^{\prime}$ and $\theta_{0 n}^{\prime \prime}$ exists. Suppose both points exist. Then (2.3.2) implies

$$
P_{\theta_{0 n}}\left(\bar{x}_{n} \leq \lambda\left(\theta_{0 n}^{\prime}\right)\right)+P_{\theta_{0 n}}\left(\bar{x}_{n} \geq \lambda\left(\theta_{0 n}^{\prime}\right)\right) \geq \frac{1}{2} \alpha_{n} .
$$


Since

$$
\begin{aligned}
& P_{\theta_{\text {On }}}\left(\bar{x}_{n} \leq \lambda\left(\theta_{\text {On }}^{\prime}\right)\right)= \\
& =\int_{\left(-\infty, \lambda\left(\theta_{O n}^{\prime}\right)\right]} \exp \left[n\left(\theta_{O n}-\theta_{O n}^{\prime}\right) x-n \psi\left(\theta_{O n}\right)+n \psi\left(\theta_{O n}^{\prime}\right)\right\} d \bar{P}_{\theta_{O n}^{\prime}}^{n}(x) \\
& \leq \int_{\left(-\infty, \lambda\left(\theta_{O n}^{\prime}\right)\right]} \exp \left\{n\left(\theta_{O n}-\theta_{O n}^{\prime}\right) \lambda\left(\theta_{O n}^{\prime}\right)-n \psi\left(\theta_{O n}\right)+n \psi\left(\theta_{O n}^{\prime}\right)\right\} d \mathrm{P}_{\theta_{O n}^{\prime}}^{-n} \quad(x) \\
& =\exp \left\{-\mathrm{nI}\left(\theta_{O_{\mathrm{n}}^{\prime}}^{\prime}, \theta_{O_{\mathrm{n}}}\right)\right\} P_{\theta_{O \mathrm{n}}^{\prime}}\left(\overline{\mathrm{x}}_{\mathrm{n}} \leq \lambda\left(\theta_{O_{\mathrm{n}}^{\prime}}^{\prime}\right)\right) \\
& \leq \exp \left(-n d_{n}\right),
\end{aligned}
$$

and similarly

$$
P_{\theta_{O n}}\left(\bar{x}_{n} \geq \lambda\left(\theta_{O_{n}}\right)\right) \leq \exp \left\{-n I\left(\theta_{O n}, \theta_{O n}\right)\right\}=\exp \left(-n d_{n}\right),
$$

it follows by substituting in (2.3.3) that $\frac{1}{2} \alpha_{n} \leq 2 \exp \left(-n d_{n}\right)$. If only one of the points $\theta_{O_{n}}^{\prime}$ and $\theta_{O_{n}}^{\prime \prime}$ exists, the same argument yields that $\frac{1}{2} \alpha_{n} \leq$ $\exp \left(-n d_{n}\right)$. This completes the proof of case a).

b) Let $P_{\theta_{0}}\left(\phi_{n}^{L R}\left(\bar{x}_{n}\right)>0, \bar{x}_{n} \in \Lambda\right)<\frac{1}{2} \alpha_{n}$ for all $\theta_{0} \in \theta_{0}$. Since $E_{\theta_{0 n}} \phi_{n}^{L R}\left(\bar{x}_{n}\right) \geq$ $9 \alpha_{n} / 10$ for some $\theta_{0 n} \in \theta_{0}$ it follows that in this case

$$
P_{\theta_{0 n}}\left(\phi_{n}^{L R}\left(\bar{x}_{n}\right)>0, \bar{x}_{n} \notin \Lambda\right) \geq \frac{2 \alpha_{n}}{5} \text {, }
$$

and hence

$$
\begin{aligned}
& \max \left\{P_{\theta_{0 n}}\left(L_{n}\left(\bar{x}_{n}\right) \leq \exp \left(-n d_{n}\right), \bar{x}_{n} \leq \underline{\lambda}\right),\right. \\
& \left.\quad P_{\theta_{0 n}}\left(L_{n}\left(\bar{x}_{n}\right) \leq \exp \left(-n d_{n}\right), \bar{x}_{n} \geq \bar{\lambda}\right)\right\} \geq \frac{\alpha_{n}}{5} .
\end{aligned}
$$

Suppose $\bar{\lambda}<\infty$ and

(2.3.4) $\quad P_{\theta_{0 n}}\left(L_{n}\left(\bar{x}_{n}\right) \leq \exp \left(-n d_{n}\right), \bar{x}_{n} \geq \bar{\lambda}\right) \geq \frac{\alpha_{n}}{5}$

(in the other case the proof is quite similar).

For $x \geq \bar{\lambda}$ the function $\theta x-\psi(\theta)$ is increasing in $\theta$ (take $\theta_{1}<\theta_{2}$, then 
$\left.\theta_{1} x-\psi\left(\theta_{1}\right)-\theta_{2} x+\psi\left(\theta_{2}\right) \leq\left(\theta_{1}-\theta_{2}\right) \lambda\left(\theta_{2}\right)-\psi\left(\theta_{1}\right)+\psi\left(\theta_{2}\right)=-I\left(\theta_{2}, \theta_{1}\right)<0\right)$. This implies that for $\mathbf{x} \geq \bar{\lambda}$

$$
\sup _{\theta \in \Theta} \exp \{n \theta x-n \psi(\theta)\}=\lim _{\theta \uparrow \bar{\theta}} \exp \{n \theta x-n \psi(\theta)\} .
$$

Thus by $(2.3 .4)$

$$
\begin{aligned}
\alpha_{n} / 5 \leq & P_{\theta_{0 n}}\left(L_{n}\left(\bar{x}_{n}\right) \leq \exp \left(-n d_{n}\right), \bar{x}_{n} \geq \bar{\lambda}\right) \\
\leq & \int_{\left\{x \geq \bar{\lambda}, L_{n}(x) \leq \exp \left(-n d_{n}\right)\right\} \sup _{\theta_{0} \in \theta_{0}} \exp \left\{n \theta_{0} x-n \psi\left(\theta_{0}\right)\right\} d \mu^{-n}(x)} \\
\leq & \exp \left(-n d_{n}\right) \\
& \int_{\left\{x \geq \bar{\lambda}, L_{n}(x) \leq \exp \left(-n d_{n}\right)\right\}} \sup _{\theta \in \Theta} \exp \{n \theta x-n \psi(\theta)\} d \mu^{-n}(x) \\
\leq & \exp \left(-n d_{n}\right) \int_{[\bar{\lambda}, \infty)} 1 i \bar{m}_{\bar{\theta}} \exp \{n \theta x-n \psi(\theta)\} d \mu^{-n}(x) \\
= & \exp \left(-n d_{n}\right) \lim _{\theta \uparrow \bar{\theta}} \int_{[\bar{\lambda}, \infty)} \exp \{n \theta x-n \psi(\theta)\} d \mu^{-n}(x) \\
\leq & \exp \left(-n d_{n}\right),
\end{aligned}
$$

where we have applied the monotone convergence theorem.

This completes the proof of the lemma. $\square$

The result of part a) of the proof can be written as

$$
\sup _{\theta_{0} \in \theta_{0}} P_{\theta_{0}}\left(\bar{x}_{n} \in \Lambda, I\left(\lambda^{-1}\left(\bar{x}_{n}\right), \theta_{0}\right) \geq d_{n}\right) \leq 5 \exp \left(-n d_{n}\right) \text {. }
$$

This result is related to theorem 6 in EFRON and TRUAX (1968). However, where Efron and Truax have a simple hypothesis (called $\theta_{1}$ in [8]) we have an arbitrary set $\theta_{0}$. The price we have to pay for this, is the constant 5 where Efron and Truax have a factor $\mathrm{n}^{-\frac{1}{2}}$ (see also example 2.3.1). Moreover, we allowed $d_{n}$ to go to zero, which is excluded in [8].

In chapter III another form of the inequality (2.3.1) will be derived in the k-dimensional case. There we discuss the relationship with the result of Efron and Truax in more detail. 


\subsection{THE MP TEST OF $\mathrm{H}_{0}$ AGAINST A SIMPLE ALTERNATIVE}

Let $\left\{\theta_{n}\right\}$ be a sequence in $\theta_{1} \wedge\left\{c l \theta_{0}\right\}^{c}$. If $\left\{\theta ; \theta \in \theta_{0}, \theta<\theta_{n}\right\}$ is nonempty, define

$$
\underline{\theta}_{-\mathrm{n}}^{0}=\sup \left\{\theta ; \theta \in \theta_{0}, \theta<\theta_{\mathrm{n}}\right\}
$$

Similarly, if $\left\{\theta ; \theta \in \Theta_{0}, \theta>\theta_{n}\right\}$ is non-empty, define

$$
\bar{\theta}_{\mathrm{n}}^{0}=\inf \left\{\theta ; \theta \in \Theta_{0} ; \theta>\theta_{\mathrm{n}}\right\}
$$

Now we describe for several cases the form of the MP test of $\mathrm{H}_{0}$ against the simple alternative $\theta_{n}$.

If $\left\{\theta ; \theta \in \Theta_{0}, \theta>\theta_{n}\right\}=\varnothing$, the critical function $\phi_{n}^{+}$of the size- $\alpha_{n}$ MP test of $\mathrm{H}_{0}: \theta \in \theta_{0}$ against $\theta=\theta_{\mathrm{n}}$ has the form (cf. LEHMANN (1959), section 3.3)

$$
\phi_{n}^{+}(x)= \begin{cases}1 & > \\ \gamma_{n} & \text { if } x=c_{n}, \\ 0 & <\end{cases}
$$

where $\gamma_{n}$ and $c_{n}$ are determined by $E_{\theta_{n}} \phi_{n}^{+}\left(\bar{x}_{n}\right)=\alpha_{n}$.

If on the other hand $\left\{\theta ; \theta \in \theta_{0}, \theta<\theta_{n}\right\}=\varnothing$ the MP test is of the form

$$
\phi_{n}^{+}(x)= \begin{cases}1 & < \\ \gamma_{n} & \text { if } x=c_{n} \\ 0 & >\end{cases}
$$

where $\gamma_{n}$ and $c_{n}$ are determined by $E_{\bar{\theta}_{n}^{0}} \phi_{n}^{+}\left(\bar{x}_{n}\right)=\alpha_{n}$.

Finally, if both $\left\{\theta ; \theta \in \theta_{0}, \theta<\theta_{n}\right\}$ and $\left\{\theta ; \theta \in \theta_{0}, \theta>\theta_{n}\right\}$ are non-empty the MP test is of the form (cf. LEHMANN (1959), section 3.7)

$$
\phi_{n}^{+}(x)= \begin{cases}1 & c_{n}^{\prime}<x<c_{n}^{\prime \prime} \\ \gamma_{n}^{\prime} \text { if } & x=c_{n}^{\prime} \\ \gamma_{n}^{\prime \prime} & x=c_{n}^{\prime \prime} \\ 0 & x \notin\left[c_{n}^{\prime}, c_{n}^{\prime \prime}\right]\end{cases}
$$

where $\gamma_{n}^{\prime}, \gamma_{n}^{\prime \prime}$ and $c_{n}^{\prime} \leq c_{n}^{\prime \prime}$ are determined by

$$
\mathrm{E}_{\underline{\theta}_{\mathrm{n}}^{0}} \phi_{\mathrm{n}}^{+}\left(\overline{\mathrm{x}}_{\mathrm{n}}\right)=\mathrm{E}_{\bar{\theta}_{\mathrm{n}} \mathrm{O}} \phi_{\mathrm{n}}^{+}\left(\overline{\mathrm{x}}_{\mathrm{n}}\right)=\alpha_{\mathrm{n}} .
$$

In the sequel $\phi_{n}^{+}$will always denote the critical function of the 
size- $\alpha_{\mathrm{n}}$ MP test of $\mathrm{H}_{0}: \theta \in \theta_{0}$ against the simple alternative $\theta=\theta_{\mathrm{n}}$.

\subsection{THE MAIN THEOREM}

We start with an example showing that

$$
\lim _{n \rightarrow \infty} R_{n}(\theta)=0 \quad \text { uniformly on } \theta_{1} \text {, }
$$

is not necessarily true.

EXAMPLE 2.5.1. Let $\mathrm{x}_{1}, \mathrm{x}_{2}, \ldots$ be independent Bernoulli random variables and let $\mathrm{H}_{0}: \theta=0, \mathrm{H}_{1}: \theta \neq 0$ and $\alpha_{\mathrm{n}}=2^{-\mathrm{n}}$. The LR test has the following form:

$$
\phi_{n}^{L R}(x)=\left\{\begin{array}{ll}
\frac{1}{2} & \text { if } x=1 \text { or } x=0 \\
0 & \text { otherwise }
\end{array}\right. \text {. }
$$

Choose a sequence $\theta_{n}=\log \left(n^{2}-1\right)$, corresponding to probability of success $1-n^{-2}$. Then the size- $\alpha_{n}$ MP test of $H_{0}$ against $\theta=\theta_{n}$ is given by

$$
\phi_{n}^{+}(x)=\left\{\begin{array}{ll}
1 & \text { if } x=1 \\
0 & \text { otherwise }
\end{array}\right. \text {. }
$$

Thus $R_{n}\left(\theta_{n}\right)=\beta_{n}^{+}\left(\theta_{n}\right)-\beta_{n}^{L R}\left(\theta_{n}\right)=\left(1-n^{-2}\right)^{n}-\frac{1}{2}\left(1-n^{-2}\right)^{n}-\frac{1}{2}\left(n^{-2}\right)^{n}$, and $\lim _{n \rightarrow \infty} R_{n}\left(\theta_{n}\right)=\frac{1}{2}$.

Using the degeneration of $\bar{x}_{n}$ for this sequence of alternatives we obtain in this example a not uniformly vanishing shortcoming. Consequent$1 y$, the level of significance $\alpha_{n}$ has to be chosen extremely small. In [18] OOSTERHOFF and VAN ZWET have constructed another example to show that

(2.5.1) is not necessarily true. Considering a very complicated hypothesis they avoid an extremely small $\alpha_{n}$.

In view of the preceding example conditions have to be introduced to ensure the validity of (2.5.1). The fact that the shortcoming is the difference of the power function of the MP test and the LR test, suggests to choose conditions in terms of either the MP test or the LR test. It turns out that sufficient conditions in terms of the MP test are very complicated and hard to verify, because they depend on the particular sequence of alternatives considered. We therefore abandoned this approach. 
A convenient condition in terms of the LR test is

$$
\alpha_{n}=o(1) \exp \left(-n d_{n}\right) \quad \text { as } n \rightarrow \infty \text {. }
$$

In theorem 2.5.1 will be shown that (2.5.2) implies (2.5.1).

Comparing (2.5.2) with lemma 2.3.1 it is seen that the constant 5 appearing in (2.3.1) is replaced by a factor $O(1)$ in (2.5.2). The examples 2.3.1 and 2.5.1 show that the inequality $\alpha_{n} \leq 5 \exp \left(-n_{n}\right)$ is not strong enough a condition.

Although (2.5.2) is not easily verified in particular cases, some corollaries will be presented in section 2.6 , covering the cases $A, B$ and C mentioned in section 2.1 .

THEOREM 2.5.1. If the critical value $d_{n}$ of the LR test satisfies (2.5.2), then the shortcoming of the LR test tends to zero uniformly on $\theta_{1}$.

PROOF. To prove (2.5.1) we suppose to the contrary that lim $\sup _{n \rightarrow \infty} R_{n}\left(\theta_{n}\right)>0$ for some sequence $\left\{\theta_{n}\right\}$ in $\theta_{1}$. Without loss of generality we assume that $\theta_{n} \in \theta_{1} \wedge\left\{c l \theta_{0}\right\}^{C}$ and $R_{n}\left(\theta_{n}\right) \geq \varepsilon$ for all $n$ and some $\varepsilon>0\left(R_{n}(\theta) \leq \alpha_{n}\right.$ for all $\theta \in c l \theta_{0}$ and $\left.\lim _{n \rightarrow \infty} \alpha_{n}=0\right)$. Let $\left\{\theta_{m}\right\}$ be a subsequence of $\left\{\theta_{n}\right\}$.

Using the notation of section 2.4 we distinguish the following three cases:

a. $\left\{\theta ; \theta \in \Theta_{0}, \theta<\theta_{\mathrm{m}}\right\} \neq \varnothing$ and $\left\{\theta ; \theta \in \Theta_{0}, \theta>\theta_{\mathrm{m}}\right\} \neq \varnothing$ for all $\mathrm{m}$ with subcases a1. $\min \left\{I\left(\theta, \underline{-}_{\mathrm{m}}^{0}\right), I\left(\theta, \bar{\theta}_{\mathrm{m}}^{0}\right)\right\}>\mathrm{d}_{\mathrm{m}}$ for some $\theta \in\left(\underline{-}_{\mathrm{m}^{\prime}}^{0}, \bar{\theta}_{\mathrm{m}}^{0}\right)$ and all $\mathrm{m}$, a2. $\min \left\{I\left(\theta, \underline{-}_{-m}^{0}\right), I\left(\theta, \bar{\theta}_{m}^{0}\right)\right\} \leq d_{m}$ for every $\theta \in\left(\underline{\theta}_{-m}^{0}, \bar{\theta}_{m}^{0}\right)$ and all $\mathrm{m}$. b. $\left\{\theta ; \theta \in \Theta_{0}, \theta<\theta_{m}\right\}=\varnothing$ for all $\mathrm{m}$.

c. $\left\{\theta ; \theta \in \theta_{0}, \theta>\theta_{m}\right\}=\varnothing$ for all $\mathrm{m}$.

In all these cases we shall obtain a contradiction. As we can pick at least one subsequence $\left\{\theta_{m}\right\}$ of $\left\{\theta_{n}\right\}$ satisfying the assumptions of one of these cases, this proves the theorem.

CASE a1. In this case the LR test has part of its critical region in the interval $\left(\lambda\left(\underline{\theta}_{m}^{0}\right), \lambda\left(\bar{\theta}_{m}^{0}\right)\right)$. Define $d_{m}^{\prime}$ and $d_{m}^{\prime \prime}$ by

$$
I\left(\lambda^{-1}\left(d_{m}^{\prime}\right), \underline{\theta}_{-m}^{0}\right)=I\left(\lambda^{-1}\left(d_{m}^{\prime \prime}\right), \bar{\theta}_{m}^{0}\right)=d_{m}
$$

and

$$
\lambda\left(\underline{\theta}_{-m}^{0}\right) \leq d_{m}^{\prime}<d_{m}^{\prime \prime} \leq \lambda\left(\bar{\theta}_{m}^{0}\right) \text { for all m. }
$$

From $R_{m}\left(\theta_{m}\right)=E_{\theta_{m}}\left\{\phi_{m}^{+}\left(\bar{x}_{m}\right)-\phi_{m}^{L R}\left(\bar{x}_{m}\right)\right\} \geq \varepsilon$ for all $m$ we derive that 


$$
\begin{aligned}
& \max \left\{E_{\theta_{m}} \phi_{m}^{+}\left(\bar{x}_{m}\right){ }_{\left[c_{m}^{\prime}, d_{m}^{\prime}\right]}\left(\bar{x}_{m}\right),\right. \\
& E_{\theta_{m}} \phi_{m}^{+}\left(\bar{x}_{m}\right){ }^{1}\left[d_{m}^{\prime \prime}, c_{m}^{\prime \prime}\right]
\end{aligned}
$$

Assume without essential loss of generality

$$
E_{\theta_{m}}\left\{\phi_{m}^{+}\left(\bar{x}_{m}\right) 1_{\left[c_{m}^{\prime}, d_{m}^{\prime}\right]}\left(\bar{x}_{m}\right)\right\} \geq \frac{1}{2 \varepsilon \varepsilon} \quad \text { for all } m
$$

Then we have, for all $\mathrm{m}$,

$$
\begin{aligned}
& \alpha_{\mathrm{m}}=\underset{\underline{\theta}_{\mathrm{m}}}{\mathrm{E}} \phi_{\mathrm{m}}^{+}\left(\overline{\mathrm{x}}_{\mathrm{m}}\right) \\
& =\int \phi_{m}^{+}(x) \exp \left\{m\left(\underline{\theta}_{-m}^{0}-\theta_{m}\right) x-m \psi\left(\underline{\theta}_{m}^{0}\right)+m \psi\left(\theta_{m}\right)\right\} d \bar{P}_{\theta}^{-m}(x) \\
& \geq \int_{\left[c_{m}^{\prime}, d_{m}^{\prime}\right]} \phi_{m}^{+}(x) \exp \left\{m\left(\theta_{-m}^{0}-\theta_{m}\right) x-m \psi\left({ }_{-m}^{0}\right)+m \psi\left(\theta_{m}\right)\right\} d P_{\theta_{m}}^{-m}(x) \\
& \geq \int_{\left[c_{m^{\prime}}^{\prime} d_{m}^{\prime}\right]_{m}} \phi_{m}^{+}(x) \exp \left\{m\left(\theta_{-m}^{0}-\theta_{m}\right) d_{m}^{\prime}-m \psi\left(\theta_{-m}^{0}\right)+m \psi\left(\theta_{m}\right)\right\} d P_{\theta_{m}^{-m}}(x) \\
& =\exp \left\{-m I\left(\lambda^{-1}\left(d_{m}^{\prime}\right), \theta_{-m}^{0}\right)+m I\left(\lambda^{-1}\left(d_{m}^{\prime}\right), \theta_{m}\right)\right\} \times \\
& \times E_{\theta_{m}} \phi_{m}^{+}\left(\bar{x}_{m}\right){ }_{\left[c_{m}^{\prime}, d_{m}^{\prime}\right]}\left(\bar{x}_{m}\right) \\
& \geq \varepsilon / 2 \exp \left(-\mathrm{md}_{\mathrm{m}}\right):
\end{aligned}
$$

a contradiction to $(2 \cdot 5.2)$.

CASE a2. Now the intersection of the interval $\left(\lambda\left(\theta_{-m}^{0}\right), \lambda\left(\bar{\theta}_{m}^{-0}\right)\right)$ and the critical region of the LR test is empty. Define $x_{m}^{0}$ in $\left(\lambda\left(\underline{\theta}_{m}^{0}\right), \lambda\left(\bar{\theta}_{m}^{0}\right)\right)$ by $I\left(\lambda^{-1}\left(x_{m}^{0}\right), \theta_{m}^{0}\right)=I\left(\lambda^{-1}\left(x_{m}^{0}\right), \bar{\theta}_{m}^{0}\right)$.

$$
\begin{gathered}
\text { Since } I\left(\lambda^{-1}\left(x_{m}^{0}\right), \theta_{-m}^{0}\right) \leq d_{m}, \text { and } \beta_{m}^{+}\left(\theta_{m}\right) \geq R_{m}\left(\theta_{m}\right) \geq \varepsilon, \\
\max \left\{E_{\theta_{m}} \phi_{m}^{+}\left(\bar{x}_{m}\right) 1{ }_{\left[c_{m}^{\prime}, x_{m}^{0}\right]}\left(\bar{x}_{m}\right),\right. \\
\left.E_{\theta_{m}} \phi_{m}^{+}\left(\bar{x}_{m}\right) 1{ }_{\left[x_{m}^{0}, c_{m}^{\prime \prime}\right]}^{0}\left(\bar{x}_{m}\right)\right\} \geq \frac{1}{2} \varepsilon \quad \text { for all } m .
\end{gathered}
$$

Assume without loss of generality 


$$
\left.\mathrm{E}_{\theta_{\mathrm{m}}} \phi_{\mathrm{m}}^{+}\left(\overline{\mathrm{x}}_{\mathrm{m}}\right) 1 \mathrm{c}_{\mathrm{m}}^{\prime}, \mathrm{x}_{\mathrm{m}}^{0}{ }^{0} \overline{\mathrm{x}}_{\mathrm{m}}\right) \geq \frac{1}{2} \varepsilon \quad \text { for all } \mathrm{m} \text {. }
$$

Note that in this case $c_{m}^{\prime} \leq x_{m}^{0}$. Then we have, for all $m$,

$$
\begin{aligned}
& \alpha_{\mathrm{m}}=\underset{\mathrm{\theta}_{-\mathrm{m}}}{\mathrm{E}} \phi_{\mathrm{m}}^{+}\left(\overline{\mathrm{x}}_{\mathrm{m}}\right) \\
& \geq \int_{\left[c_{m^{\prime}}^{\prime} x_{m}^{0}\right]} \phi_{m}^{+}(x) \exp \left\{m\left(\theta_{-m}^{0}-\theta_{m}\right) x-m \psi\left(\theta_{m}^{0}\right)+m \psi\left(\theta_{m}\right)\right\} d \bar{P}_{\theta_{m}^{-m}}^{-m}(x) \\
& \geq \exp \left\{-\mathrm{mI}\left(\lambda^{-1}\left(x_{m}^{0}\right), \theta_{-m}^{0}\right)+m I\left(\lambda^{-1}\left(x_{m}^{0}\right), \theta_{m}\right)\right\} \times \\
& \left.\times E_{\theta_{m}} \phi_{m}^{+}\left(\bar{x}_{m}\right) 1 c_{m}^{\prime}, x_{m}^{0}\right]\left(\bar{x}_{m}\right) \\
& \geq \varepsilon / 2 \exp \left(-\mathrm{md}_{\mathrm{m}}\right) \text {, }
\end{aligned}
$$

in contradiction to $(2 \cdot 5.2)$.

CASE b. Note that in this case $\bar{\theta}_{\mathrm{m}}^{0}$ coincides with $\underline{\theta}_{0}$. Define

(2.5.3) $\quad f_{m}=\left\{\psi\left(\underline{\theta}_{0}\right)-\psi\left(\theta_{m}\right)-d_{m}\right\}\left(\underline{\theta}_{0}-\theta_{m}\right)^{-1}$.

Then the following implication holds

$(2.5 .4) \quad x<f_{m} \Rightarrow L_{m}(x)<\exp \left(-m_{m}\right)$.

To prove this, first note that $\mathrm{f}_{\mathrm{m}}<\lambda\left(\underline{\theta}_{0}\right)$, since $\mathrm{f}_{\mathrm{m}}-\lambda\left(\underline{\theta}_{0}\right)=$ $\left\{\psi\left(\underline{\theta}_{0}\right)-\psi\left(\theta_{m}\right)-d_{m}-\left(\underline{\theta}_{0}-\theta_{m}\right) \lambda\left(\underline{\theta}_{0}\right)\right\}\left(\underline{\theta}_{0}-\theta_{m}\right)^{-1}=\left(\underline{\theta}_{0}-\theta_{m}\right)^{-1}\left\{-I\left(\underline{\theta}_{0}, \theta_{m}\right)-d_{m}\right\}<0$. Hence

$$
\sup _{\theta_{0} \epsilon \Theta_{0}} \exp \left\{m \theta_{0} x-m \psi\left(\theta_{0}\right)\right\}=\exp \left\{m \underline{\theta}_{0} x-m \psi\left(\underline{\theta}_{0}\right)\right\} \quad \text { for } x<f_{m} .
$$

This implies, for every $x<\mathrm{f}_{\mathrm{m}}$,

$$
\begin{aligned}
L_{m}(x) & =\frac{\sup _{\theta_{0} \in \Theta_{0}} \exp \left\{m \theta_{0} x-m \psi\left(\theta_{0}\right)\right\}}{\sup _{\theta \in \Theta} \exp \{m \theta x-m \psi(\theta)\}} \\
& \leq \exp \left\{m \underline{\theta}_{0} x-m \psi\left(\underline{\theta}_{0}\right)-m \theta_{m} x+m \psi\left(\theta_{m}\right)\right\}<
\end{aligned}
$$




$$
\begin{aligned}
& <\exp \left\{m\left(\underline{\theta}_{0}-\theta_{m}\right) f_{m}-m \psi\left(\underline{\theta}_{0}\right)+m \psi\left(\theta_{m}\right)\right\} \\
& =\exp \left(-m d_{m}\right),
\end{aligned}
$$

establishing (2.5.3). In other words: for every $x<f_{m}$ is $\phi_{m}^{L R}(x)=1$. But since $R_{m}\left(\theta_{m}\right) \geq \varepsilon$, it follows that

$$
E_{\theta_{m}} \phi_{m}^{+}\left(\bar{x}_{m}\right) 1_{\left[f_{m}, \infty\right)}\left(\bar{x}_{m}\right) \geq \varepsilon
$$

Hence

$$
\begin{aligned}
& \alpha_{m}=E_{\theta_{0}} \phi_{m}^{+}\left(\bar{x}_{m}\right) \\
& \geq \int_{\left[f_{m^{\prime}}, \infty\right)} \phi_{m}^{+}(x) \exp \left\{m\left(\underline{\theta}_{0}-\theta_{m}\right) x-m \psi\left(\underline{\theta}_{0}\right)+m \psi\left(\theta_{m}\right)\right\} d \bar{P}_{\theta_{m}}^{-m}(x) \\
& \geq \int_{\left[f_{m^{\prime}}, \infty\right)} \phi_{m}^{+}(x) \exp \left\{m\left(\underline{\theta}_{0}-\theta_{m}\right) x-m \psi\left(\underline{\theta}_{0}\right)+m \psi\left(\theta_{m}\right)\right\} d \bar{P}_{\theta_{m}}^{-m}(x) \\
& =\exp \left(-m d_{m}\right) E_{\theta_{m}} \phi_{m}^{+}\left(\bar{x}_{m}\right) 1_{\left[f_{m}, \infty\right)}\left(\bar{x}_{m}\right) \geq \varepsilon \exp \left(-m d_{m}\right),
\end{aligned}
$$

again in contradiction to $(2.5 .2)$.

CASE c. The same line of argument that we used in case b again yields a contradiction.

This completes the proof of the theorem. $\square$

Inspection of the proof of theorem 2.5.1 shows that we have in fact proved

$$
R_{n}(\theta) \leq 2 \alpha_{n} e^{n d_{n}}
$$

for all $\mathrm{n}$ and all $\theta \in \theta_{1}$. Hence $\mathrm{R}_{\mathrm{n}}(\theta) \rightarrow 0$ if either (2.5.2) holds true or if $R_{n}(\theta)=o\left(\alpha_{n} e^{n d}\right)$ as $n \rightarrow \infty$. The following example shows that the latter possibility may indeed occur.

EXAMPLE 2.5.2. Let $\mathrm{x}_{1}, \mathrm{x}_{2}, \ldots$ be independent Bernoulli random variables and let $\mathrm{H}_{0}: \theta \leq 0, \mathrm{H}_{1}: \theta>0$ and $\alpha_{\mathrm{n}}=2^{-\mathrm{n}}$. Both the LR test and the MP test of $\mathrm{H}_{0}$ against $\theta=\theta_{n}>0$ has the following form: reject $\mathrm{H}_{0}$ iff $\overline{\mathrm{x}}_{\mathrm{n}}=1$. Hence $\lim _{n \rightarrow \infty} R_{n}(\theta)=0$ uniformly on $\theta_{1}$ and yet $\alpha_{n}=\exp \left(-n d_{n}\right)$. 


\subsection{SOME PARTICULAR CASES}

With the help of theorem 2.5.1 we investigate the cases A, B and C mentioned in section 2.1 .

COROLLARY 2.6.1. If $\theta_{0} \subset \mathrm{K}$, where $\mathrm{K}$ is a compact subset of int $\theta$, and if $I<I_{0}$ exists such that

(2.6.1) $\quad \alpha_{n} \geq \exp (-n I) \quad$ for all sufficiently large $n$,

where

(2.6.2) $\left.I_{0}=\underset{\theta+\underline{\theta}}{\min \{\lim } I\left(\theta, \theta_{0}\right), \lim _{\theta \uparrow \bar{\theta}} I\left(\theta, \theta_{0}\right)\right\}$,

then $\lim _{n \rightarrow \infty} R_{n}(\theta)=0$ uniformly on $\theta_{1}$.

(Note that $I_{0}$ is well defined.)

PROOF. We verify condition (2.5.2). To this end we inspect the proof of lemma 2.3.1 a little more carefully.

Consider case a) of the proof. There it is shown that

$$
P_{\theta_{0 n}}\left(\bar{x}_{n} \leq \lambda\left(\theta_{0 n}^{\prime}\right)\right)+P_{\theta_{0 n}}\left(\bar{x}_{n} \geq \lambda\left(\theta_{0 n}^{\prime \prime}\right)\right) \geq \frac{1}{2} \alpha_{n} \quad(n=1,2, \ldots)
$$

(2.3.3), where $\theta_{\text {On }} \in \theta_{0}$ satisfies

$$
\begin{gathered}
P_{\theta_{O n}}\left(\phi_{n}^{L R}\left(\bar{x}_{n}\right)>0, \bar{x}_{n} \in \Lambda\right) \geq \frac{1}{2} \alpha_{n^{\prime}} \\
\text { and } I\left(\theta_{\text {On }}^{\prime}{ }^{\prime} \theta_{O n}\right)=I\left(\theta_{0 n}^{\prime \prime} \theta_{O n}\right)=d_{n}\left(\theta_{O n}<\theta_{O n}<\theta_{O n}^{\prime \prime}\right) \cdot \\
\text { Assuming without loss of generality }
\end{gathered}
$$

(2.6.3) $\quad P_{\theta_{0 n}}\left(\overline{\mathrm{x}}_{\mathrm{n}} \leq \lambda\left(\theta_{0 \mathrm{n}}^{\prime}\right)\right) \geq \frac{1}{4} \alpha_{n} \quad$ for $\mathrm{n}=1,2, \ldots$

one finds

(2.6.4) $\quad{ }^{\frac{1}{4}} \alpha_{n} \leq P_{\theta_{0 n}}\left(\bar{x}_{n} \leq \lambda\left(\theta_{O n}^{\prime}\right)\right) \leq \exp \left\{-n I\left(\theta_{O n}^{\prime}, \theta_{O n}\right)\right\}$.

Suppose the sequence $\left\{\alpha_{n}\right\}$ satisfies the condition of the corollary and $\theta_{\text {On }} \in \mathrm{K}$ for all $\mathrm{n}$. If $\left\{\theta_{\mathrm{O}_{\mathrm{n}}}^{\prime}\right\}$ has a subsequence, which tends to the boundary of $\theta$ for $n \rightarrow \infty$, i.e. lim inf ${ }_{n \rightarrow \infty} \theta_{\text {On }}^{\prime}=\underline{\theta}$, then $\lim \sup _{n \rightarrow \infty} I\left(\theta_{0 n}^{\prime}{ }^{\prime} \theta_{0 n}\right) \geq$ $\lim _{\theta+\underline{\theta}} I\left(\theta, \theta_{0}\right) \geq I_{0}$, and hence 


$$
I\left(\theta_{\mathrm{On}_{i}}^{\prime},{ }^{\prime} \mathrm{On}_{i}\right)>\mathrm{I}+\varepsilon
$$

for some subsequence $\left\{\mathrm{n}_{i}\right\}$ and some $\varepsilon>0$.

This implies, in view of (2.6.4),

$$
\frac{1}{4} \alpha_{n_{i}} \leq \exp \left\{-n_{i}(I+\varepsilon)\right\} \quad(i=1,2, \ldots),
$$

and the rate of convergence of $\left\{\alpha_{n_{i}}\right\}$ to zero is faster than prescribed in (2.6.1).

Hence assume that $\left\{\theta_{0 \mathrm{n}}^{\prime}\right\}$ is bounded away from the boundary of $\theta$. Consequently $\sigma\left(\theta_{O_{n}}^{\prime}\right)$ and the central third moments (under $\theta_{O_{n}}^{\prime}$ ) are bounded away from zero and infinity and Liapunov's version of the central limit theorem ensures that $n^{\frac{1}{2}}\left\{\bar{x}_{n}-\lambda\left(\theta_{0 n}^{\prime}\right)\right\} \sigma\left(\theta_{0 n}^{\prime}\right)^{-1} \stackrel{D}{\rightarrow} N(0,1)$ for $n \rightarrow \infty$.

If $\left(\theta_{0 n}-\theta_{O n}^{\prime}\right) n^{\frac{1}{2}}$ is bounded, $n I\left(\theta_{O n}^{\prime},{ }_{0 n}\right)=n d_{n}$ is also bounded (see lemma 2.2.2) and (2.5.2) is trivial.

Assume therefore that

(2.6.5) $\quad\left(\theta_{0 n}-\theta_{O n}^{\prime}\right) n^{\frac{1}{2}} \rightarrow \infty \quad$ for $n \rightarrow \infty$.

By $(2.6 .3)$

$$
\begin{aligned}
& { }^{\frac{1}{4}} \alpha_{n} \leq P_{\theta_{O n}}\left(\bar{x}_{n} \leq \lambda\left(\theta_{O n}^{\prime}\right)\right) \\
& \leq \int_{\left(-\infty, \lambda\left(\theta_{O_{n}}^{\prime}\right)\right]} \exp \left\{-n I\left(\theta_{O_{n}}^{\prime}{ }^{\prime} \theta_{O n}\right)+n\left(\theta_{0 n^{-}}{ }^{-}{ }_{O n}^{\prime}\right)\left(x-\lambda\left(\theta_{O n}^{\prime}\right)\right)\right\} d \bar{P}_{\theta_{O n}^{\prime}}^{-n}(x)
\end{aligned}
$$



Hence for each $n>0$

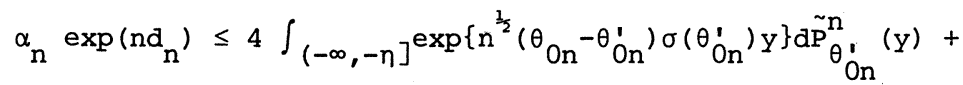

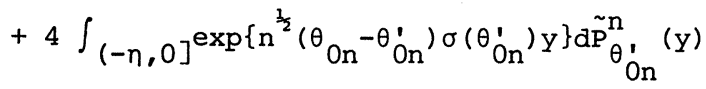

$$
\begin{aligned}
& \leq 4 \exp \left\{-n^{\frac{1}{2}}\left(\theta_{0 n}{ }^{-}{ }_{O n}^{\prime}\right) \sigma\left(\theta_{O n}^{\prime}\right) n\right\}+4 \tilde{P}_{\theta_{0 n}^{\prime}}^{n}\{(-n, 0]\} \text {. }
\end{aligned}
$$

By $(2.6 .5) \mathrm{n}^{\frac{1}{2}}\left(\theta_{0 n}{ }^{-\theta_{0 n}^{\prime}}\right) \sigma\left(\theta_{O_{n}}^{\prime}\right) \rightarrow \infty$ as $\mathrm{n} \rightarrow \infty$; now the last inequality implies

$$
\underset{n \rightarrow \infty}{\lim \sup } \alpha_{n} \exp \left(\mathrm{nd}_{n}\right) \leq 0+2 n \text {, }
$$


and thus $\lim _{n \rightarrow \infty} \alpha_{n} \exp \left(n d_{n}\right)=0$ or $\alpha_{n}=o(1) \exp \left(-n d_{n}\right)$ as $n \rightarrow \infty$.

Now consider part b) of the proof of lemma 2.3.1; again assume that $P_{\theta_{0 n}}\left(L_{n}\left(\bar{x}_{n}\right) \leq \exp \left(-n d_{n}\right), \bar{x}_{n} \geq \bar{\lambda}\right) \geq \alpha_{n} / 5$ (2.3.4). By (2.3.5)

$$
\begin{aligned}
\alpha_{n} / 5 & \leq \int_{\left\{x \geq \bar{\lambda}, L_{n}(x) \leq \exp \left(-n d_{n}\right)\right\}} \sup _{\theta_{0} \in \theta_{0}} \exp \left\{n \theta_{0} x-n \psi\left(\theta_{0}\right)\right\} d \bar{\mu}^{-n}(x) \\
& \leq \exp \left(-n d_{n}\right) .
\end{aligned}
$$

Condition (2.6.1) yields

$$
\underset{n \rightarrow \infty}{\lim \sup _{n}} d_{n} \leq I_{0} \leq \lim _{\theta \uparrow \bar{\theta}} I\left(\theta, \bar{\theta}_{0}\right)
$$

Choose $\varepsilon>0$ such that $I+\varepsilon<I_{0}$; then we have, for $\mathrm{n}$ sufficiently large and $x \geq \bar{\lambda}$,

$$
\begin{aligned}
d_{n} \leq & \lim \theta+\bar{\theta} \\
= & \lim \left(\theta, \bar{\theta}_{0}\right)-\varepsilon \\
& \theta \uparrow \bar{\theta} \\
\leq & \left.\lim _{\theta \uparrow \bar{\theta}}\left\{\psi\left(\bar{\theta}_{0}\right)-\psi(\theta)+\left(\theta-\bar{\theta}_{0}\right) \lambda(\theta)\right\}-\varepsilon(\theta)+\left(\theta-\bar{\theta}_{0}\right) x\right\}-\varepsilon,
\end{aligned}
$$

and hence, for $x \geq \bar{\lambda}$,

$$
\begin{aligned}
\sup _{\theta_{0} \in \Theta_{0}}\left\{\theta_{0} x-\psi\left(\theta_{0}\right)\right\} & =\bar{\theta}_{0} x-\psi\left(\bar{\theta}_{0}\right) \\
& \leq \lim _{\theta \uparrow \bar{\theta}}\{\theta x-\psi(\theta)\}-d_{n}-\varepsilon .
\end{aligned}
$$

Then we have

$$
\begin{aligned}
\alpha_{n} / 5 & \int_{\left\{x \geq \bar{\lambda}, L_{n}(x) \leq \exp \left(-n d_{n}\right)\right\}} \sup _{\theta_{0} \in \Theta_{0}} \exp \left\{n \theta_{0} x-n \psi\left(\theta_{0}\right)\right\} d \mu^{-n}(x) \\
& \leq \int_{\left\{x \geq \bar{\lambda}, L_{n}(x) \leq \exp \left(-n d_{n}\right)\right\}}^{\exp \left(-n d_{n}-n \varepsilon\right)} \lim _{\theta \uparrow \bar{\theta}} \exp \{n \theta x-n \psi(\theta)\} d \mu^{-n}(x) \leq
\end{aligned}
$$




$$
\begin{aligned}
& \leq \exp \left(-n d_{n}-n \varepsilon\right) \lim _{\theta+\bar{\theta}} \int_{\left\{x \geq \bar{\lambda}, L_{n}(x) \leq \exp \left(-n d_{n}\right)\right\}} \exp \{n \theta x-n \psi(\theta)\} d \mu^{-n}(x) \\
& \leq \exp \left(-n d_{n}-n \varepsilon\right) .
\end{aligned}
$$

So we have once more $\alpha_{n}=o(1) \exp \left(-n d_{n}\right)$. Application of theorem 2.5.1 completes the proof.

COROLLARY 2.6.2. If $\theta_{1} \subset \mathrm{K}$, where $\mathrm{K}$ is a compact subset of int $\theta$, $\lim _{n \rightarrow \infty} R_{n}(\theta)=0$ uniformly on $\theta_{1}$.

Replacing $\theta_{0}$ by $\mathrm{cl} \theta_{0} \wedge \mathrm{K}$ and using the proof of corollary 2.6.1, again one can derive $\alpha_{n}=o(1) \exp \left(-n d_{n}\right)$. Application of theorem 2.5.1 then completes the proof. We omit the details, since the result can also be obtained as an immediate consequence of theorem 2.7.1.

In the preceding corollaries we have put some rather strong conditions on $\theta_{0}$ or $\theta_{1}$. These conditions ensured, that the critical region of the LR test is bounded away from the boundary of $\Lambda$, implying that the distribution of the standardized sample mean tends to a (standard) normal distribution for suitable translated parameter values. By putting strong conditions on the moments of $x_{i}$, we obtain the same result as the following corollary shows.

COROLLARY 2.6.3. Let, for $\theta \in$ int $\theta$, the variance $\sigma^{2}(\theta)$ of $\mathrm{x}_{i}$ be bounded away from zero and the absolute third central moment of $\mathrm{x}_{i}$ be bounded above. Then $\lim _{n \rightarrow \infty} R_{n}(\theta)=0$ uniformly on $\theta_{1}$.

PROOF. The boundedness of the absolute third central moment of $x_{i}$ implies that $\sigma^{2}(\theta)$ is also bounded above on int $\theta$. Moreover, if $\theta \epsilon \theta$ is a boundary point of $\theta$ and $\lambda(\theta)$ is finite, then the variance and the third central moment at $\theta$ are also finite, and the variance is bounded away from zero (the proof is similar to the proof of lemma 2.2.1).

We inspect the proof of lemma 2.3.1. By Liapunov's theorem $n^{\frac{1}{2}}\left\{\bar{x}_{n}-\lambda\left(\theta_{O n}^{\prime}\right)\right\} \sigma\left(\theta_{O n}^{\prime}\right)^{-1}$ is asymptotic standard normal for each sequence $\left\{\theta_{0 \mathrm{n}}^{\prime}\right\}$ in $\theta^{*}$.

Consider case a) of the proof of lemma 2.3.1. By the same line of argument, used in the first part of the proof of corollary 2.6.1, (but 
immediately invoking the before mentioned asymptotic normality) yields $\alpha_{n}=o(1) \exp \left(-n d_{n}\right)$.

Now consider case b). Assume $\bar{\lambda}<\infty$ and

$$
P_{\theta_{0 n}}\left(L_{n}\left(\bar{x}_{n}\right) \leq \exp \left(-n d_{n}\right), \bar{x}_{n} \geq \bar{\lambda}\right) \geq \alpha_{n} / 5
$$

(the other case is quite similar). Since $d_{n}>0$, we have $\bar{\theta}_{0}<\bar{\theta}$. Let $0<t<\bar{\theta}-\bar{\theta}_{0}$. For every $x \geq \bar{\lambda}$ and each $\varepsilon_{n}>0$ one has

$$
\begin{aligned}
L_{n}\left(x+\varepsilon_{n}\right) & =\frac{\sup _{\theta_{0} \epsilon \theta_{0}} \exp \left\{n \theta_{0}\left(x+\varepsilon_{n}\right)-n \psi\left(\theta_{0}\right)\right\}}{\sup _{\theta \in \Theta} \exp \left\{n \theta\left(x+\varepsilon_{n}\right)-n \psi(\theta)\right\}} \\
& =\frac{\exp \left\{n \bar{\theta}_{0}\left(x+\varepsilon_{n}\right)-n \psi\left(\bar{\theta}_{0}\right)\right\}}{\lim \exp \left\{n \theta\left(x+\varepsilon_{n}\right)-n \psi(\theta)\right\}} \\
& \leq \frac{\exp \left\{n \bar{\theta}_{0}\left(x+\varepsilon_{n}\right)-n \psi\left(\bar{\theta}_{0}\right)\right\}}{\lim \exp \left\{n \theta x+n\left(\bar{\theta}_{0}+t\right) \varepsilon_{n}-n \psi(\theta)\right\}} \\
& =\exp \left(-n t \varepsilon_{n}\right) L_{n}(x) .
\end{aligned}
$$

Define $x_{n}^{0}=\inf \left\{x ; x \geq \bar{\lambda}, L_{n}(x) \leq \exp \left(-n d_{n}\right)\right\}$ and choose an arbitrary $\varepsilon>0$. Since $x \geq x_{n}^{0}+\varepsilon n^{-\frac{1}{2}}$ implies $L_{n}(x) \leq \exp \left(-n t^{\frac{1}{2} \varepsilon n^{-\frac{1}{2}}}\right) L_{n}\left(x_{n}^{0}+\frac{1}{2} \varepsilon n^{-\frac{1}{2}}\right) \leq$ $\exp \left(-\frac{1}{2} t \varepsilon n^{\frac{1}{2}}-n_{n}\right)$, it follows that

$$
\begin{aligned}
\alpha_{n} / 5 & \leq P_{\theta_{0 n}}\left(\bar{x}_{n} \geq x_{n}^{0}+\varepsilon n^{-\frac{1}{2}}\right) \\
& +\int_{\left\{x_{n}^{0} \leq x \leq x_{n}^{0}+\varepsilon n^{-\frac{1}{2}}, L_{n}(x) \leq \exp \left(-n d_{n}\right)\right\}} \exp \left\{n \theta_{0 n} x-n \psi\left(\theta_{0 n}\right)\right\} d \bar{\mu}^{-n}(x) \\
& \leq \int_{\left\{x \geq x_{n}^{0}+\varepsilon n^{-\frac{1}{2}}\right\}} \exp \left\{-\frac{1}{2} t \varepsilon n^{\frac{1}{2}}-n d_{n}\right\}{ }_{\theta \uparrow \bar{\theta}} \exp \{n \theta x-n \psi(\theta)\} d \mu^{-n}(x)+ \\
& +\int_{\left\{x_{n}^{0} \leq x \leq x_{n}^{0}+\varepsilon n^{-\frac{1}{2}}, L_{n}(x) \leq \exp \left(-n d_{n}\right)\right\}^{\theta+\bar{\theta}}} \exp \left(-n d_{n}\right) \lim (n-n(\theta)\} d \mu^{-n}(x) \\
& \leq \exp \left(-\frac{1}{2} \operatorname{ten}^{\frac{1}{2}}-n d_{n}\right)+\exp \left(-n d_{n}\right) \lim _{\theta \uparrow \bar{\theta}} P_{\theta}\left(x_{n}^{0} \leq \bar{x}_{n} \leq x_{n}^{0}+\varepsilon n^{-\frac{1}{2}}\right)
\end{aligned}
$$


Therefore

$$
\begin{aligned}
& \underset{n \rightarrow \infty}{\lim \sup _{n}} \alpha_{n} \exp \left(n d_{n}\right) \leq \\
& \leq \underset{n \rightarrow \infty}{\lim \sup } \lim _{\theta \uparrow \bar{\theta}} P_{\theta}\left(x_{n}^{0} \leq \bar{x}_{n} \leq x_{n}^{0}+\varepsilon n^{-\frac{1}{2}}\right) \\
& \leq \lim \sup _{n \rightarrow \infty} \lim _{\theta+\bar{\theta}} P_{\theta}\left[\frac{x_{n}^{0}-\lambda(\theta)}{\sigma(\theta)} n^{\frac{1}{2}} \leq \frac{\bar{x}_{n}-\lambda(\theta)}{\sigma(\theta)} n^{\frac{1}{2}} \leq \frac{x_{n}^{0}-\lambda(\theta)}{\sigma(\theta)} n^{\frac{1}{2}}+\frac{\varepsilon}{\sigma(\theta)}\right] \\
& \leq \frac{\varepsilon}{\inf \{\sigma(\theta) ; \theta \in \theta\}} .
\end{aligned}
$$

Since $\varepsilon$ was arbitrary chosen and $\inf \{\sigma(\theta) ; \theta \in \Theta\}>0$, we have

$\lim _{n \rightarrow \infty} \alpha_{n} \exp \left(n d_{n}\right)=0$. Application of theorem 2.5 .1 completes the proof.

EXAMPLE 2.6.1. The family of normal distributions with expectation $\theta \in \mathbb{R}$ and unit variance satisfies the conditions of corollary 2.6.3, and hence $R_{n}(\theta) \rightarrow 0$ uniformly on $\theta_{1}$, irrespective of the hypothesis $\theta_{0}$ and the rate of convergence of $\left\{\alpha_{n}\right\}$.

\subsection{UNIFORM CONVERGENCE ON COMPACT SUBSETS OF INT $\theta$}

In this section we show that, without any restrictions on the sets $\Theta_{0}$ and $\theta_{1}$, the sequence $\left\{\alpha_{n}\right\}$ and the moments of $x_{i}, R_{n}(\theta) \rightarrow 0$ as $n \rightarrow \infty$ uniformly on the intersection of $\theta_{1}$ with a compact subset of int $\theta$. This result is an extension of corollary 2.6.2.

THEOREM 2.7.1. Let $\mathrm{K}$ be an arbitrary compact subset of int $\theta$. Then $\lim _{n \rightarrow \infty} R_{n}(\theta)=0$ uniformly on $K \wedge \theta_{1}$.

PROOF. It is sufficient to show that $\lim _{n \rightarrow \infty} R_{n_{2}}\left(\theta_{n}\right)=0$ for any sequence $\left\{\theta_{n}\right\}$ in $K \wedge \theta_{1}$. Let $\left\{\theta_{n}\right\}$ be such a sequence. Then $\sigma^{2}\left(\theta_{n}\right)$ is bounded away from zero and infinity, and $\tilde{\mathrm{P}}_{\theta_{\mathrm{n}}}^{\mathrm{n}} \rightarrow_{\mathrm{w}} \mathrm{N}(0,1)$ by Liapunov's central limit theorem. Suppose to the contrary that lim $\sup _{n \rightarrow \infty} R_{n}\left(\theta_{n}\right)>0$. Without loss of generality assume that $R_{n}\left(\theta_{n}\right)>\varepsilon$ for all $n=1,2, \ldots$ and some $\varepsilon>0$. To obtain a contradiction, we modify the proof of theorem 2.5.1. The order relation $\alpha_{n}=o(1) \exp \left(-n d_{n}\right)$ as $n \rightarrow \infty$ in that proof is now replaced by

$$
\alpha_{n} \leq 5 \exp \left(- \text { nd }_{n}\right) \quad n=1,2, \ldots
$$


cf. lemma 2.3.1.

First consider case a1 in the proof of theorem 2.5.1. With the same definitions of $d_{n}^{\prime}$ and $d_{n}^{\prime \prime}$ assume

$$
E_{\theta_{n}}\left\{\phi_{n}^{+}\left(\bar{x}_{n}\right) 1_{\left[c_{n}^{\prime}, d_{n}^{\prime}\right]}\left(\bar{x}_{n}\right)\right\} \geq \frac{1}{2 \varepsilon} \text { for all } n,
$$

or

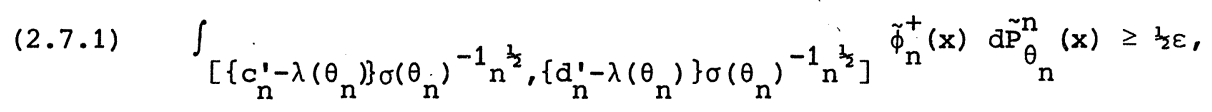

where $\tilde{\phi}_{n}^{+}(x)=\phi_{n}^{+}\left(\lambda\left(\theta_{n}\right)+n^{-\frac{1}{2}} \sigma\left(\theta_{n}\right) x\right)$. Noting that $\tilde{P}_{\theta_{n}}^{n} \rightarrow_{w} N(0,1)$, it follows that for all sufficiently large $n$ the distribution function of $\tilde{P}_{\theta_{n}}^{n}$ does not have jumps larger than $\varepsilon / 10$. In combination with (2.7.1) this yields the existence of $b_{n}>0$ such that for all sufficiently large $n$

(2.7.2) $\int_{\left[\left\{c_{n}^{\prime}-\lambda\left(\theta_{n}\right)\right\} \sigma\left(\theta_{n}\right)^{-1} n^{\frac{1}{2}},\left\{d_{n}^{\prime}-b_{n}-\lambda\left(\theta_{n}\right)\right\} \sigma\left(\theta_{n}\right)^{-1} n^{\frac{1}{2}}\right)} \tilde{\phi}_{n}^{+}(x) d \tilde{P}_{\theta_{n}^{n}}^{n}(x)>\frac{\varepsilon}{10}$,

and

$$
\int\left[\left\{d_{n}^{\prime}-b_{n}-\lambda\left(\theta_{n}\right)\right\} \sigma\left(\theta_{n}\right)^{-1} n^{\frac{1}{2}},\left\{d_{n}^{\prime}-\lambda\left(\theta_{n}\right)\right\} \sigma\left(\theta_{n}\right)^{-1} n^{\frac{1}{2}}\right] \tilde{\phi}_{n}^{+}(x) d \tilde{P}_{\theta_{n}^{n}}(x)>\frac{\varepsilon}{10}
$$

From the second inequality and the fact that $\sigma\left(\theta_{n}\right)$ is bounded away from zero, we derive that

$(2.7 .3) \quad \underset{n \rightarrow \infty}{\lim i n f} b_{n} n^{\frac{3}{2}}>0$

For $\alpha_{n}$ we then have the following inequality

$(2.7 .4) \quad \alpha_{n}=E_{\theta_{-n}^{0}} \phi_{n}^{+}\left(\bar{x}_{n}\right)$

$$
\begin{aligned}
& =\int \exp \left\{n\left(\underline{\theta}_{n}^{0}-\theta_{n}\right)\left(x-\lambda\left(\theta_{n}\right)\right)-n I\left(\theta_{n}{ }^{0}-_{n}^{0}\right)\right\} \phi_{n}^{+}(x) d P_{\theta_{n}}^{-n}(x) \\
& \geq \exp \left\{-n I\left(\theta_{n^{\prime}} \underline{-}_{n}^{0}\right)\right\} \int \quad \exp \left\{n^{\frac{1}{2}}\left(\underline{\theta}_{n}^{0}-\theta_{n}\right) \sigma\left(\theta_{n}\right) x\right\} \tilde{\phi}_{n}^{+}(x) d \tilde{P}_{\theta_{n}}^{n}(x) . \\
& \quad\left[\left\{c_{n}^{\prime}-\lambda\left(\theta_{n}\right)\right\} \sigma\left(\theta_{n}\right)^{-1} n^{\frac{1}{2}},\left\{d_{n}^{\prime}-b_{n}-\lambda\left(\theta_{n}\right)\right\} \sigma\left(\theta_{n}\right)^{-1} n^{\frac{3}{2}}\right)
\end{aligned}
$$


Suppose $\lim _{i \rightarrow \infty} n_{i}^{\frac{1}{2}}\left(\theta_{n_{i}}-{ }^{0} n_{i}\right)<\infty$ for some subsequence $\left\{n_{i}\right\}$; then $\lim _{i \rightarrow \infty} n_{i}^{\frac{1}{2}}\left(\theta_{n_{i}}-\theta^{0} n_{i}\right) \sigma\left(\theta_{n_{i}}\right)<\infty$, and hence by (2.7.2) and $\tilde{P}_{\theta_{n}}^{n} \rightarrow_{w} N(0,1)$ the integral in (2.7.4) is bounded away from zero for the subsequence $\left\{n_{i}\right\}$. Now consider $\exp \left\{-n_{i} I\left(\theta_{n_{i}}, \underline{\theta}^{0}\right)\right\}$. Since $\theta_{n_{i}} \in K(i=1,2, \ldots)$ and $\lim _{i \rightarrow \infty}\left(\theta_{n_{i}}-\theta_{-}^{0}\right)=0$, there is a compact subset $K^{\prime} \subset$ int $\theta$ such that $\underline{\theta}_{n_{i}}^{0} \in K^{\prime}$ for sufficiently large $i$. But then is $\left\{n_{i} I\left(\theta_{n_{i}}, \underline{\theta}_{n_{i}}^{0}\right)\right\}$ also bounded above and (2.7.4) implies that $\left\{\alpha_{n_{i}}\right\}$ is bounded away from zero, in contradiction to $\alpha_{n} \rightarrow 0$. It follows that

$$
\lim _{n \rightarrow \infty} n^{\frac{1}{2}}\left(\theta_{n}-\underline{\theta}^{0}\right)=\infty \text {. }
$$

Hence from $(2 \cdot 7.4)$ :

$$
\begin{aligned}
\alpha_{n} & \geq \frac{\varepsilon}{10} \exp \left\{-n I\left(\theta_{n}, \theta_{n}^{0}\right)-n\left(\theta_{n}-\theta_{-n}^{0}\right)\left(d_{n}^{\prime}-\lambda\left(\theta_{n}\right)\right)+n\left(\theta_{n}-\theta_{-n}^{0}\right) b_{n}\right\} \\
& \geq \frac{\varepsilon}{10} \exp \left\{-n I\left(\lambda^{-1}\left(d_{n}^{\prime}\right), \theta_{n}^{0}\right)+n I\left(\lambda^{-1}\left(d_{n}^{\prime}\right), \theta_{n}\right)+n\left(\theta_{n}-{ }_{-n}^{0}\right) b_{n}\right\} \\
& \geq \frac{\varepsilon}{10} \exp \left\{-n d_{n}+n\left(\theta_{n}-{ }_{-n}^{0}\right) b_{n}\right\} .
\end{aligned}
$$

Combining (2.7.3), (2.7.5) and (2.7.6) a contradiction is obtained to $\alpha_{n} \leq 5 \exp \left(-n d_{n}\right)$. This completes the proof of case a1.

Case a2 of the proof of theorem 2.5.1 can be treated similarly.

Next we consider case $b$. In the course of the proof of theorem

2.5 .1 it was shown, cf. $(2.5 .3)$ and $(2.5 .5)$, that

$$
E_{\theta_{n}} \phi_{n}^{+}\left(\bar{x}_{n}\right) 1_{\left[f_{n} ; \infty\right)}\left(\bar{x}_{n}\right) \geq \varepsilon,
$$

or

$$
\int_{\left[\left\{f_{n}-\lambda\left(\theta_{n}\right)\right\} \sigma\left(\theta_{n}\right)^{-1} n^{\frac{1}{2}}, \infty\right)} \tilde{\phi}_{n}^{+}(x) d \tilde{P}_{\theta_{n}}^{n}(x) \geq \varepsilon,
$$

where again $\tilde{\phi}_{n}^{+}(x)=\phi_{n}^{+}\left(\lambda\left(\theta_{n}\right)+n^{-\frac{1}{2}} \sigma\left(\theta_{n}\right) x\right)$. As in case a1 there exist numbers $b_{n}>0$ such that for sufficiently large $n$

$$
\int\left[\left\{f_{n}-\lambda\left(\theta_{n}\right)\right\} \sigma\left(\theta_{n}\right)^{-1} n^{\frac{1}{2}},\left\{f_{n}-\lambda\left(\theta_{n}\right)+b_{n}\right\} \sigma\left(\theta_{n}\right)^{-1} n^{\frac{1}{2}}\right) \tilde{\phi}_{n}^{+}(x) d \tilde{P}_{\theta_{n}^{n}}^{n}(x)>\frac{\varepsilon}{10},
$$




$$
\int_{\left[\left\{f_{n}-\lambda\left(\theta_{n}\right)+b_{n}\right\} \sigma\left(\theta_{n}\right)^{-1} n^{\frac{1}{2}}, \infty\right)} \tilde{\phi}_{n}^{+}(x) d \tilde{P}_{\theta_{n}^{n}}^{n}(x)>\frac{\varepsilon}{10} .
$$

From the first inequality we derive

$$
(2.7 .7) \quad \underset{n \rightarrow \infty}{\lim \inf } n^{\frac{1}{2}} b_{n}>0
$$

Repeating the argument of case a1 we can conclude that

$$
\text { (2.7.8) } \quad \lim _{n \rightarrow \infty} n^{\frac{1}{2}}\left(\bar{\theta}_{0}-\theta_{n}\right)=\infty \text {, }
$$

and hence

$$
\text { (2.7.9) } \begin{aligned}
\alpha_{n} & =E_{\bar{\theta}_{0}} \phi_{n}^{+}\left(\bar{x}_{n}\right) \\
& =\exp \left\{-n I\left(\theta_{n}, \bar{\theta}_{0}\right)\right\} \int \phi_{n}^{+}(x) \exp \left\{-n\left(\theta_{n}-\bar{\theta}_{0}\right)\left(x-\lambda\left(\theta_{n}\right)\right)\right\} d \bar{P}_{\theta_{n}^{n}}^{-n}(x) \\
& \geq \exp \left\{-n I\left(\theta_{n}, \bar{\theta}_{0}\right)\right\} \int \quad\left[\left\{\tilde{\phi}_{n}^{+}(x) \exp \left\{-n_{n}^{\frac{1}{2}}\left(\theta_{n}\right)+\bar{\theta}_{0}\right) \sigma\left(\theta_{n}\right) x\right\} d_{n} \tilde{P}_{\theta_{n}}^{n}(x)\right. \\
& \geq \frac{\varepsilon}{10} \exp \left\{-n I\left(\theta_{n}, \bar{\theta}_{0}\right)+n\left(\bar{\theta}_{0}-\theta_{n}\right)\left(f_{n}-\lambda\left(\theta_{n}\right)\right)+n\left(\bar{\theta}_{0}-\theta_{n}\right) b_{n}\right\} \\
& \geq \frac{\varepsilon}{10} \exp \left\{-n d_{n}+n\left(\bar{\theta}_{0}-\theta_{n}\right) b_{n}\right\} .
\end{aligned}
$$

Combination of $(2.7 .7),(2.7 .8)$ and $(2.7 .9)$ a contradiction is obtained to $\alpha_{n} \leq 5 \exp \left(-\right.$ nd $\left._{n}\right)$.

The same method of case $b$ also leads to a contradiction in case $c$.

This completes the proof of the theorem.

\subsection{POINTWISE CONVERGENCE}

Theorem 2.7.1 obviously implies the much weaker result $\lim _{n \rightarrow \infty} R_{n}(\theta)=0$, pointwise for each $\theta \in \theta_{1} \wedge$ int $\theta$. It remains to consider the boundary points of $\theta$. We first present a useful lemma of independent interest.

LEMMA 2.8.1. Let $\mathrm{x}_{1}, \mathrm{x}_{2}, \ldots$ be i.i.d. non-degenerate random variables, and $s_{n}=\sum_{i=1}^{n} x_{i}(n=1,2, \ldots)$. Let $\left\{n_{n}\right\}$ be some sequence, satisfying $n_{n}=o\left(n^{\frac{1}{2}}\right)$ as $\mathrm{n} \rightarrow \infty$. Denote by $\mathrm{J}_{\mathrm{n}}$ the set of intervals of length $n_{\mathrm{n}}$. Then 
(2.8.1) $\lim _{n \rightarrow \infty} \sup _{I_{n} \in J_{n}} \operatorname{Pr}\left(S_{n} \in I_{n}\right)=0$

PROOF. The result of the lemma can be obtained by application of an inequality of KOLMOGOROV, stated in [12] and proved in [13].

THEOREM 2.8.2. For all $\theta \in \theta_{1}$ it holds that $\lim _{n \rightarrow \infty} R_{n}(\theta)=0$.

PROOF. We only have to consider boundary points of $\theta$. Let $\underline{\theta} \epsilon \theta$ (the case $\bar{\theta} \epsilon \theta$ can be treated similarly). If $\underline{\theta}_{0}=\underline{\theta}$, continuity of the power function of the MP test implies $\beta_{n}^{+}(\underline{\theta}) \leq \alpha_{n} \rightarrow 0$ as $n \rightarrow \infty$, and hence $\lim _{n \rightarrow \infty} R_{n}(\underline{\theta})=0$.

Assume therefore $\underline{\theta}<\underline{\theta}_{0}$. Suppose lim $\sup _{n \rightarrow \infty} R_{n}(\underline{\theta})>0$. Without loss of generality assume $R_{n}(\underline{\theta}) \geq \varepsilon>0$ for all $n$. Defining

$$
f_{n}=\left\{\psi\left(\underline{\theta}_{0}\right)-\psi(\underline{\theta})-d_{n}\right\}\left\{\underline{\theta}_{0}-\underline{\theta}\right\}^{-1}
$$

we have the implication: $x<f_{n} \Rightarrow \phi_{n}^{L \cdot R}(x)=1$ (for a proof see (2.5.4) et sq.) and hence $E_{\underline{\theta}} \phi_{n}^{+}\left(\bar{x}_{n}\right) 1_{\left[f_{n}, \infty\right)}\left(\bar{x}_{n}\right) \geq \varepsilon$ for all $n$. By lemma 2.8 .1 . $\lim _{n \rightarrow \infty} P_{\underline{\theta}}\left(\bar{x}_{n} \in\left[f_{n}, f_{n}+n^{-\frac{3}{4}}\right)\right)=0$, and therefore

$$
\mathrm{E}_{\underline{\theta}} \phi_{\mathrm{n}}^{+}\left(\overline{\mathrm{X}}_{\mathrm{n}}\right) 1_{\left[\mathrm{f}_{\mathrm{n}}+\mathrm{n}^{-\frac{3}{4}}, \infty\right)}\left(\overline{\mathrm{X}}_{\mathrm{n}}\right) \geq \varepsilon / 2
$$

for all sufficiently large $n$.

But this implies, for large $n$,

$$
\begin{aligned}
\alpha_{n} & =E_{\underline{\theta}_{0}} \phi_{n}^{+}\left(\bar{x}_{n}\right) \\
& \geq \int \phi_{n}^{+}(x) \exp \left\{n\left(\underline{\theta}_{0}-\underline{\theta}\right) x-n \psi\left(\underline{\theta}_{0}\right)+n \psi\left(\underline{\theta}_{n}\right)\right\} d \bar{P}_{\underline{\theta}}^{n}(x) \\
& \geq \frac{1}{2} \varepsilon \exp \left\{n\left(\underline{\theta}_{0}-\underline{\theta}^{-\frac{3}{4}}\right)\left(\mathrm{f}_{n}+n^{-\frac{3}{4}}\right)-n \psi\left(\underline{\theta}_{0}\right)+n \psi(\underline{\theta})\right\} \\
\geq & \frac{1}{2} \varepsilon \exp \left(-n d_{n}\right) \exp \left\{n^{\frac{1}{4}}\left(\underline{\theta}_{0}-\underline{\theta}\right)\right\} .
\end{aligned}
$$

Since on the other hand, by lemma 2.3.1, $\alpha_{n} \leq 5 \exp \left(-n d_{n}\right)$, we have obtained a contradiction and therefore $\lim _{n \rightarrow \infty} R_{n}(\underline{\theta})=0$. 

CHAPTER III

THE k-PARAMETER CASE

\subsection{INTRODUCTION}

In chapter II we have described in detail the behaviour of the shortcoming in the one-parameter exponential family model. In this chapter we present some generalizations of these results to the k-parameter case.

We represent a k-parameter exponential family by

$$
\mathrm{dP}_{\theta}(\mathbf{x})=\exp \left\{\theta^{\prime} \mathrm{x}-\psi(\theta)\right\} \mathrm{d} \mu(\mathbf{x}), \quad \mathrm{x} \in \mathbb{R}^{\mathrm{k}},
$$

where $\mu$ is a non-degenerate probability measure and $0 \epsilon$ int $\theta$. For each $\mathrm{n} \in \mathbb{N}$ consider the testing problem $\mathrm{H}_{0}: \theta \in \theta_{0}$ against $\mathrm{H}_{1}: \theta \in \theta_{1}$ at level $\alpha_{n}$ with the available observations $x_{1}, \ldots, x_{n}$, where $\lim _{n \rightarrow \infty} \alpha_{n}=0$. Except for section $3.8 \theta_{1}=\theta-\theta_{0}$. We investigate the behaviour of the shortcoming $R_{n}(\theta)$ of the size- $\alpha_{n}$ LR test as $n \rightarrow \infty$.

The basic results of chapter II are lemma 2.3.1 and theorem 2.5.1. By lemma 2.3.1 $\alpha_{n} \leq 5 \exp \left(-n d_{n}\right)$, where $d_{n}$ is the critical value of the LR test, in the one-parameter case. In the k-parameter model such a nice inequality is not generally true as the following example shows:

EXAMPLE 3.1 .1 . Let $\mathrm{Y}_{1}, \mathrm{Y}_{2}, \ldots$ be i.i.d. random variables with a normal $\mathrm{N}\left(\xi, \sigma^{2}\right)$ distribution. The family of distributions constitutes a two-parameter exponential family with $\theta=\left(\xi \sigma^{-2}, \frac{1}{2}\left(1-\sigma^{-2}\right)\right)$ and $x_{i}=\left(x_{i}^{(1)}, x_{i}^{(2)}\right)=\left(Y_{i}, y_{i}^{2}\right)$. We consider the testing problem $\mathrm{H}_{0}: \xi=0, \sigma^{2}=1$ against $\mathrm{H}_{1}: \xi \neq 0$ or $\sigma^{2} \neq 1$. The LR test of this problem has the following form: reject $\mathrm{H}_{0}$ if $\mathrm{n}^{-1} \sum_{i=1}^{\mathrm{n}} \mathrm{Y}_{i}^{2}-\log \mathrm{n}^{-1} \sum_{i=1}^{\mathrm{n}}\left(\mathrm{Y}_{i}-\overline{\mathrm{Y}}_{\mathrm{n}}\right)^{2}>1+2 \mathrm{~d}_{\mathrm{n}}$ (in the notation of $(1.2 .10)$ ). Hence, if $\mathrm{Y}_{i}$ is normal $\mathrm{N}(0,1)$ distributed,

$$
\begin{aligned}
\alpha_{n} & \geq \operatorname{Pr}\left(n^{-1} \sum_{i=1}^{n}\left(Y_{i}-\bar{Y}_{n}\right)^{2}<\exp \left(-2 d_{n}-1\right)\right) \\
& =\int_{0}^{n \exp \left(-2 d_{n}-1\right)} \exp \left(-\frac{1}{2} y\right) y^{\frac{n-1}{2}-1}\left[2^{\frac{n-1}{2}} \Gamma\left(\frac{n-1}{2}\right)\right]^{-1} d y .
\end{aligned}
$$


Let $d_{n} \rightarrow \infty$ so fast that $n \exp \left(-2 d_{n}-1\right) \rightarrow 0$. Then, for sufficiently large $n$,

$$
\text { (3.1.2) } \begin{aligned}
\alpha_{n} & \geq \frac{1}{2} \int_{0}^{n \exp \left(-2 d_{n}-1\right) \frac{n-1}{2}-1}\left[2^{\frac{n-1}{2}} \Gamma\left(\frac{n-1}{2}\right)\right]^{-1} d y \\
& \geq 1 / 3 n^{-\frac{1}{2}} \exp \left(-n d_{n}+d_{n}\right),
\end{aligned}
$$

by an application of Stirling's formula.

Choosing for example $d_{n} \geq n$ (3.1.2) contradicts the statement $\alpha_{n} \leq\left(n d_{n}\right)^{p} \exp \left(-n d_{n}\right)$ for every fixed $p$. So even in the case of a simple hypothesis an inequality like $\alpha_{n} \leq 5 \exp \left(-n d_{n}\right)$ does not hold.

Moreover, the condition $\alpha_{n}=o(1) \exp \left(-n d_{n}\right)$, as $n \rightarrow \infty$, appearing in theorem 2.5.1 is not satisfied in either "regular" k-dimensional cases. This is demonstrated by the next. example.

EXAMPLE 3.1.2. Let $\left(\mathrm{X}_{1}, \mathrm{Y}_{1}\right),\left(\mathrm{X}_{2}, \mathrm{Y}_{2}\right), \ldots$ be i.i.d. random variables with a normal $N\left(\xi, I_{2}\right)$ distribution, where $\xi \in \mathbb{R}^{2}$ and $I_{2}$ is the $2 \times 2$ identity matrix. Consider the testing problem $\mathrm{H}_{0}: \xi=0$ against $\mathrm{H}_{1}: \xi \neq 0$. It is easy to see that the LR test rejects $H_{0}$ if $\bar{x}_{n}^{2}+\bar{Y}_{n}^{2}>2 d_{n}$. Hence, under $H_{0}$, $\alpha_{\mathrm{n}}=\operatorname{Pr}\left(\overline{\mathrm{x}}_{\mathrm{n}}^{2}+\overline{\mathrm{Y}}_{\mathrm{n}}^{2}>2 \mathrm{~d}_{\mathrm{n}}\right)=\exp \left(-\mathrm{nd}_{\mathrm{n}}\right)$.

A natural generalization of $\alpha_{n}=o(1) \exp \left(-n d_{n}\right)$ to the $k$-dimensional case is $\alpha_{n}=o(1)\left(n d_{n}\right)(k-1) / 2 \exp \left(-n d_{n}\right)$. However, the implication $\alpha_{n}=o(1)\left(n d_{n}\right)(k-1) / \frac{n}{2} \exp \left(-n d_{n}\right) \Rightarrow \lim _{n \rightarrow \infty} R_{n}(\theta)=0$ uniformly in $\theta$ is not necessarily true. To show this we present the following example.

EXAMPLE 3.1.3. The measure $\mu$ is defined as $\mu\left(i_{1}, \ldots, i_{k}\right)=2^{-k}$ for all $\left(i_{1}, \ldots, i_{k}\right)$ with $i_{j}=0$ or $1(j=1, \ldots, k) \cdot x_{1}, x_{2}, \ldots$ are $i . i . d$. random vectors with distribution given by (3.1.1) with $\mu$ defined as above.

The hypothesis $\mathrm{H}_{0}: \theta=(0, \ldots, 0)$ is tested against $\mathrm{H}_{1}: \theta \neq(0, \ldots, 0)$ at level $\alpha_{n}=2^{-n k}$. It is easy to verify that the LR test has the following form:

$$
\phi_{n}^{L R}\left(x^{(1)}, \ldots, x^{(k)}\right)= \begin{cases}2^{-k} & \text { if }\left(x^{(1)}, \ldots, x^{(k)}\right)=\left(i_{1}, \ldots, i_{k}\right), \\ & \text { where } i_{j}=0 \text { or } 1(j=1, \ldots, k) \\ 0 & \text { otherwise }\end{cases}
$$

and $d_{n}=k \log 2$ (cf. $\left.(1 \cdot 2 \cdot 10)\right)$.

Consider a particular sequence $\left\{\theta_{n}\right\}$ in $\theta$ defined by $\theta_{n}=(2 \log n, \ldots, 2 \log n)$. The MP test of $H_{0}$ against the simple alternative 
$\theta=\theta_{n}$ of size $\alpha_{n}=2^{-n k}$ is given by

$$
\phi_{n}^{+}\left(x^{(1)}, \ldots, x^{(k)}\right)=\left\{\begin{array}{ll}
1 & \text { if }\left(x^{(1)}, \ldots, x^{(k)}\right)=(1, \ldots, 1) \\
0 & \text { otherwise }
\end{array} .\right.
$$

Since $P_{\theta_{n}}\left(\left(\bar{x}_{n}^{(1)}, \ldots, \bar{x}_{n_{k}}^{(k)}\right)=\left(i_{1}, \ldots, i_{k}\right)\right) \rightarrow 0$ for $\left(i_{1}, \ldots, i_{k}\right) \neq(1, \ldots, 1)$, we have $R_{n}\left(\theta_{n}\right)=\left(1-2^{-k}\right) P_{\theta_{n}}\left(\left(\bar{x}_{n}^{(1)}, \ldots, \bar{x}_{n}^{(k)}\right)=(1, \ldots, 1)\right)+o(1)$ as $n \rightarrow \infty$. Now $\lim _{n \rightarrow \infty} P_{\theta_{n}}\left(\left(\bar{x}_{n}^{(1)}, \ldots, \bar{x}_{n}^{(k)}\right)=(1, \ldots, 1)\right)=1$ and thus $\lim _{n \rightarrow \infty} R_{n}\left(\theta_{n}\right)=$ $1-2^{-\mathrm{k}}$.

Combining $\alpha_{n}=2^{-n k}$ and $d_{n}=k \log 2$ it follows that $\alpha_{n}=\exp \left(-n d_{n}\right)=$ $o(1)\left(n_{n}\right)^{(k-1) / 2^{n}} \exp \left(-n d_{n}\right)$ for $k \geq 2$; however, $\lim _{n \rightarrow \infty} R_{n}\left(\theta_{n}\right)=1-2^{-k^{n}}>0$.

Although the preceding examples show that general results as theorem 2.5.1 do not hold in the k-dimensional case, some of the specific results of chapter II hol.d true in the k-dimensional case.

\subsection{A GENERALIZATION OF A THEOREM OF EFRON AND TRUAX}

In this section we determine a relation between $\alpha_{n}$ and $d_{n}$. To this end we generalize theorem 6 of EFRON and TRUAX (1968).

We first define a number $I(K)$ for a subset $K$ of int $\theta$ as a sort of "Kullback-Leibler information distance" of $K$ to the boundary of $\theta$. More precisely: let $\mathrm{K} \subset$ int $\theta$, then

(3.2.1) $I(K)=\sup \left\{A ;\{\theta ; I(\theta, K) \leq A\} \subset K_{A} \subset\right.$ int $\theta$, where $K_{A}$ is compact $\}$.

We now have the following

THEOREM 3.2.1. Let $\mathrm{x}_{1}, \mathrm{x}_{2}, \ldots$ be i.i.d. random vectors, distributed as in (3.1.1). Let $\mathrm{K}$ be a subset of int $\theta$. If $\varepsilon \mathrm{n}^{-1} \leq \mathrm{d}_{\mathrm{n}} \leq$ $\min \left\{\mathrm{I}(\mathrm{K})-\varepsilon, \varepsilon^{-1}\right\}$ for some $\varepsilon>0$ and all sufficiently large $\mathrm{n}$, then

$$
P_{\theta_{0}}\left(\bar{x}_{n} \notin \lambda\left\{\theta ; I\left(\theta, \theta_{0}\right)<d_{n}\right\}\right)=\left(n d_{n}\right)^{\frac{k-2}{2}} \exp \left(-n d_{n}+0(1)\right)
$$

as $\mathrm{n} \rightarrow \infty$, uniformly for $\theta_{0} \in \mathrm{K}$.

Comparing this result with theorem 6 of Efron and Truax we allow $d_{n} \rightarrow 0$ as $n \rightarrow \infty$ where Efron and Truax require $d_{n} \geq \varepsilon>0$. (Incidently the upper bound for $d_{n}$ in [8] is incorrect.) Thus we also obtain a relation 
between $\alpha_{n}$ and $\alpha_{n}$ for subexponential rates of convergence of $\alpha_{n}$ to zero. Note that theorem 3.2.1 generalizes theorem 3 of HOEFFDING (1965b) dealing with the multinomial distribution. In [8] only a sketch of proof is presented. Apart of some technical differences the most important difference between our proof and that in [8] is the application of the (multidimensional) Berry-Esseen theorem in stead of the Rvaceva-Stone theorem.

Before proving theorem 3.2.1 we present a lemma, which enables us to go from $\theta$-space to $\lambda$-space and vice versa, and to translate "KullbackLeibler information distance" into Euclidean distance and vice versa.

LEMMA 3.2.2. Consider an exponential family (3.1.1) and some compact subset $\mathrm{K}$ of int $\theta$. Then there exist positive constants $\mathrm{c}_{1}, \ldots, \mathrm{c}_{6}$, depending only on the exponential family and $\mathrm{K}$ such that for every $\theta, \xi \in \mathrm{K}(\theta \neq \xi)$

$$
\begin{aligned}
& c_{1} \leq \frac{\|\lambda(\theta)-\lambda(\xi)\|}{\|\theta-\xi\|} \leq c_{2}, \\
& c_{3} \leq \frac{I(\theta, \xi)}{\|\theta-\xi\|^{2}} \leq c_{4}, \\
& c_{5} \leq \frac{I(\theta, \xi)}{(\theta-\xi) \cdot(\lambda(\theta)-\lambda(\xi))} \leq c_{6} .
\end{aligned}
$$

PROOF. We prove $I(\theta, \xi) \geq c_{3}\|\theta-\xi\|^{2}$. The other statements can be proved in the same way. By Taylor expansion $\psi(\xi)=\psi(\theta)+(\xi-\theta) \cdot \lambda(\theta)+\frac{1}{2}(\xi-\theta) \cdot \Sigma_{\eta}(\xi-\theta)$ for some $n$ between $\xi$ and $\theta$. Let $\mathrm{K}^{*}$ be the convex hull of $\mathrm{k}$. Then

$$
\begin{aligned}
& I(\theta, \xi)=\frac{1}{2}(\xi-\theta)^{\prime} \Sigma_{\eta}(\xi-\theta) \geq\|\theta-\xi\|^{2} \operatorname{lin}_{\frac{1}{2}} \inf _{u} \|_{=1} u^{\prime} \Sigma_{\zeta} u= \\
& =c_{3}\|\theta-\xi\|^{2} \text {. } \\
& \zeta \in \mathrm{K}^{\star}
\end{aligned}
$$

PROOF OF THEOREM 3.2.1. Let $\theta_{0} \in \mathrm{K}$. By Taylor expansion about $\lambda\left(\theta_{0}\right) \quad I\left(\lambda^{-1}(x), \theta_{0}\right)=$ $\frac{1}{2}\left(x-\lambda\left(\theta_{0}\right)\right)^{\prime} \Sigma_{\theta_{0}}^{-1}\left(x-\lambda\left(\theta_{0}\right)\right)+o\left(\left\|_{x}-\lambda\left(\theta_{0}\right)\right\|^{2}\right)$ as $x \rightarrow \lambda\left(\theta_{0}\right)$. Hence $2 n I\left(\lambda^{-1}\left(\bar{x}_{n}\right), \theta_{0}\right)$ has a chi-square limit distribution, and thus the theorem holds if $\lim _{\mathrm{n} \rightarrow \infty} \mathrm{nd}_{\mathrm{n}}<\infty$ and $\mathrm{nd}_{\mathrm{n}} \geq \varepsilon>0$.

Therefore assume that $\lim _{n \rightarrow \infty} n d_{n}=\infty$. Denote by $c_{1} \cdots c_{27}$ positive constants not depending on $\mathrm{n}$.

As the first step in our proof we introduce a "lattice" $\left\{\theta_{n, i}\right\}$ on the surface $\left\{\theta_{i} I\left(\theta, \theta_{0}\right)=d_{n}\right\}$ with distance between two neighbouring points of order $n^{-\frac{1}{2}}$. The set $\left\{\theta_{i} I\left(\theta, \theta_{0}\right)=d_{n}\right\}$ is contained in 
$K_{0}=\left\{\theta ; I(\theta, K) \leq \min \left\{I(K)-\varepsilon, \varepsilon^{-1}\right\}\right\}$, a compact set in int $\theta$; hence by lemma 3.2 .2

$$
\text { (3.2.3) } \quad\left\|\theta-\theta_{0}\right\|^{2} \geq c_{1} d_{n}
$$

for every $\theta$ satisfying $I\left(\theta, \theta_{0}\right)=d_{n}$. Choose points $\theta_{n, 1}, \ldots, \theta_{n, p_{n}}$ on $\mathrm{B}_{\mathrm{n}} \stackrel{\text { def }}{=}\left\{\theta ;\left\|\theta-\theta_{0}\right\|^{2}=\mathrm{c}_{1} \mathrm{~d}_{\mathrm{n}}\right\}$ such that for all $\theta \in \mathrm{B}_{\mathrm{n}}$ there exists $\mathrm{n}$ $\theta_{n, i}$ with $\left\|\theta-\theta_{n, i}\right\| \leq n^{-\frac{n}{2}}$, and for all $i \neq j\left\|\theta_{n, i}{ }^{-\theta_{n, j}}\right\|>n^{-\frac{1}{2}}$, where $i, j=1, \ldots, p_{n}$. It is not difficult to see that such points indeed may be determined.

We estimate the number of points $p_{n}$. For $i=1, \ldots, p_{n}$ let $s_{n, i}=$ $\left\{\theta \in B_{n} ;\left\|\theta-\theta_{n, i}\right\| \leq \frac{1}{2} n^{-\frac{1}{2}}\right\}$ then $s_{n, i} \wedge s_{n, j}=\varnothing(i \neq j)$ and hence $\sum_{i=1}^{p_{n}}$ area of $s_{n, i} \leq$ area of $B_{n}$. Since area of $s_{n, i} \geq c 2^{n^{-(k-1) / 2}}$ and area of $B_{n}=c_{3} d_{n}^{(k-1) / 2}$, it follows that $p_{n} \leq c_{4}\left(n d_{n}\right)(k-1) / 2$. Considering $T_{n, i}=\left\{\theta \in B_{n} ;\left\|\theta-\theta_{n, i}\right\| \leq n^{-\frac{1}{2}}\right\}, i=1, \ldots, p_{n}$, and using the inclusion $B_{n} \subset U_{i=1}^{p_{n}} T_{n, i}$ we find $p_{n} \geq c_{5}\left(n_{n}\right)^{(k-1) / 2}$. Hence

$$
c_{5}\left(n d_{n}\right)^{\frac{k-1}{2}} \leq p_{n} \leq c_{4}\left(n d_{n}\right)^{\frac{k-1}{2}} \text {. }
$$

We define $\tilde{\theta}_{n, i}$ by $\tilde{\theta}_{n, i}=\theta_{0}+\gamma_{n, i}\left(\theta_{n, i}-\theta_{0}\right)$ and $I\left(\tilde{\theta}_{n, i}, \theta_{0}\right)=d_{n}\left(i=1, \ldots, p_{n}\right)$. By (3.2.3) $\gamma_{n, i} \geq 1$ implying that $\left\|\tilde{\theta}_{n, i}{ }^{-\tilde{\theta}} n, j\right\| \geq \theta_{n, i}{ }^{-\theta_{n, j}} \|>n^{-\frac{1}{2}}, i \neq j$, $i, j=1, \ldots, p_{n}$. We also need an upper bound for inf ${ }_{i=1}, \ldots, p_{n}\left\|\tilde{\theta}-\tilde{\theta}_{n, i}\right\|$, where $I\left(\tilde{\theta}, \theta_{0}\right)=d_{n}$. Let $\tilde{\theta} \in\left\{\theta ; I\left(\theta, \theta_{0}\right)=d_{n}\right\}$, then $\tilde{\theta}=\theta_{0}+\gamma\left(\theta-\theta_{0}\right)$, where $\theta \in B_{n}$. Then there is a $\theta_{n, i}\left(1 \leq i \leq p_{n}\right)$ such that $\left\|\theta-\theta_{n, i}\right\| \leq n^{-\frac{1}{2}}$. By lemma 3.2 .2 $\left\|\tilde{\theta}_{n, i}-\theta_{0}\right\|^{2} \leq c_{6} d_{n}$ and hence $\gamma_{n, i} \leq c_{7}$. Take a sphere with radius $c_{7} n^{-\frac{1}{2}}$ and centre $\tilde{\theta}_{n, i}$. Then the line through $\theta_{0}$ and $\theta$ intersects this sphere at a point

$$
\theta^{*}=\theta_{0}+\gamma^{*}\left(\theta-\theta_{0}\right) \text { and }\left\|\theta^{*}-\tilde{\theta}_{n, i}\right\|=c_{7} n^{-\frac{1}{2}} \text {. }
$$

Then $I\left(\theta^{*}, \theta_{0}\right)=I\left(\tilde{\theta}_{n, i}, \theta_{0}\right)+\left(\theta^{*}-\tilde{\theta}_{n, i}\right) \cdot \Sigma_{\tilde{\theta}_{n, i}}\left(\tilde{\theta}_{n, i}-\theta_{0}\right)+O\left(n^{-1}\right) \geq d_{n}-c_{8} n^{-\frac{1}{2}} d_{n}^{\frac{1}{2}}$. Consider the function $f(h)=I\left(\theta_{0}+h\left(\theta-\theta_{0}\right), \theta_{0}\right)$ for $h \geq 0$. Its derivative $\frac{d}{d h} f(h)=h\left(\theta-\theta_{0}\right) ' \Sigma_{\theta_{0}}+h\left(\theta-\theta_{0}\right)\left(\theta-\theta_{0}\right)$. For any $h \geq \frac{1}{2}$ such that $I\left(\theta_{0}+h\left(\theta-\theta_{0}\right), \theta_{0}\right)$ $<\min \left\{I(K)-\varepsilon, \varepsilon^{-1}\right\}$ is $\frac{d}{d h} f(h) \geq c_{9} d_{n}$. Since $\gamma^{*} \geq \frac{s_{2}}{2}$ for $n$ sufficiently large (in view of (3.2.3) et seq, (3.2.5) and $n d_{n} \rightarrow \infty$ ) the mean value theorem implies $I\left(\theta^{*}+c_{8} c_{9}^{-1} n^{-\frac{1}{2}} d_{n}^{-\frac{1}{2}}\left(\theta-\theta_{0}\right), \theta_{0}\right)=f\left(\gamma^{*}+c_{8} c_{9}^{-1} n^{-\frac{1}{2}} d_{n_{1}}^{-\frac{1}{2}}\right) \geq I\left(\theta^{*}, \theta_{0}\right)+$ $c_{8} c_{9}^{-1} n^{-\frac{1}{2}} d_{n}^{-\frac{1}{2}} c_{9} d_{n} \geq d_{n}$, and hence $\gamma \leq \gamma^{*}+c_{8} c_{9}^{-1} n^{-\frac{1}{2}} d_{n}^{-\frac{1}{2}}$. In the same way we find that $\gamma \geq \gamma^{*}-c_{10} n^{-\frac{1}{2}} d_{n}^{-\frac{1}{2}}$, and thus $\left\|\tilde{\theta}-\theta^{*}\right\|=\left|\gamma-\gamma^{*}\right|\left\|\theta-\theta_{0}\right\| \leq c_{11^{n}}{ }^{-\frac{1}{2}}$. 
Combining this with $\left\|\theta^{*}-\tilde{\theta}_{n, i}\right\|=c_{7} n^{-\frac{1}{2}}$ we have $\left\|\tilde{\theta}-\tilde{\theta}_{n, i}\right\| \leq c_{12} n^{-\frac{1}{2}}$.

Thus we have obtained a sort of lattice $\left\{\tilde{\theta}_{n, 1}, \ldots, \tilde{\theta}_{n, p_{n}}\right\}$ on the surface $\left\{\theta ; I\left(\theta, \theta_{0}\right)=d_{n}\right\}$ with the following two properties: for all $\tilde{\theta}$ with $I\left(\tilde{\theta}_{1}, \theta_{0}\right)=d_{n}$ there exists a $\tilde{\theta}_{n, i}$ with $\left\|\tilde{\theta}_{n, i}-\ddot{\theta}\right\| \leq c_{12^{n}}{ }^{-\frac{1}{2}}$, and $\left\|\tilde{\theta}_{n, i}-\tilde{\theta}_{n, j}\right\|>$ $n^{-\frac{1}{2}}$ for all $i \neq j$.

It will now be shown that

(3.2.6) $P_{\theta_{0}}\left(\bar{x}_{n} \notin \lambda\left\{\theta ; I\left(\theta, \theta_{0}\right)<d_{n}\right\}\right) \geq c_{13}\left(n d_{n}\right)^{\frac{k-2}{2}} \exp \left(-n d_{n}\right)$.

Therefore we carry the "lattice" over to $\lambda$-space and consider the points $\lambda\left(\tilde{\theta}_{n, 1}\right), \ldots, \lambda\left(\tilde{\theta}_{n, p_{n}}\right)$. By lemma 3.2.2 $\left\|\lambda\left(\tilde{\theta}_{n, i}\right)-\lambda\left(\tilde{\theta}_{n_{-j} j}\right)\right\|>c_{14} n^{n^{-\frac{1}{2}}}$. Consider spheres $U_{n, i}$ with centre $\lambda\left(\tilde{\theta}_{n, i}\right)$ and radius $\frac{1}{2} C 14^{n^{-\frac{x^{j}}{2}}}, i=1, \ldots, p_{n}$, then $U_{n, i} \wedge U_{n, j}=\varnothing, i \neq j$, and $U_{n, i} \subset \lambda(\theta)$ for $n$ sufficiently large. Hence

$$
\begin{aligned}
& P_{\theta_{0}}\left(\bar{x}_{\mathrm{n}} \notin \lambda\left\{\theta ; I\left(\theta, \theta_{0}\right)<d_{n}\right\}\right) \geq \\
& \sum_{i=1}^{P_{n}} P_{\theta_{0}}\left(\bar{x}_{n} \in U_{n, i}, I\left(\lambda^{-1}\left(\bar{x}_{n}\right), \theta_{0}\right) \geq d_{n}\right) .
\end{aligned}
$$

Since $\left(\tilde{\theta}_{n, i}-\theta_{0}\right){ }^{\prime} x \geq\left(\tilde{\theta}_{n, i}-\theta_{0}\right) \cdot \lambda\left(\tilde{\theta}_{n, i}\right)$ implies that $\sup _{\theta \in \Theta}\left[\psi\left(\theta_{0}\right)-\psi(\theta)+\right.$ $\left.\left(\theta-\theta_{0}\right) \cdot x\right\} \geq d_{n}$ or $I\left(\lambda^{-1}(x), \theta_{0}\right) \geq d_{n}^{\prime}$

$$
\begin{aligned}
& P_{\theta_{0}}\left(\bar{x}_{n} \in U_{n, i^{\prime}} I\left(\lambda^{-1}\left(\bar{x}_{n}\right), \theta_{0}\right) \geq d_{n}\right)= \\
& =\iint_{\left\{U_{n, i}, I\left(\lambda^{-1}(\dot{x}), \theta_{0}\right) \geq d_{n}\right\}}^{\exp \left\{-n\left(\tilde{\theta}_{n, i}{ }^{-\theta_{0}}\right) ' x+n \psi\left(\tilde{\theta}_{n, i}\right)-n \psi\left(\theta_{0}\right)\right\} d \bar{P}_{\tilde{\theta}_{n, i}^{n}}^{n}(x)} \\
& \geq \exp \left(-n d_{n}\right) \int_{\left\{U_{n, i},\left(\tilde{\theta}_{n, i}-\theta_{0}\right) ' x \geq\left(\tilde{\theta}_{n, i}-\theta_{0}\right) \cdot \lambda\left(\tilde{\theta}_{n, i}\right)\right\}} \exp \left\{-n\left(\tilde{\theta}_{n, i}-\theta_{0}\right){ }^{\prime}\left(x-\lambda\left(\tilde{\theta}_{n, i}\right)\right)\right\} d \bar{P}_{\tilde{\theta}_{n, i}}^{-n}(x) .
\end{aligned}
$$

By the k-dimensional Berry-Esseen inequality there exists a constant

$c_{15}>0$ such that for any $c_{16}>0$

$$
\begin{aligned}
P_{\tilde{\theta}_{n, i}}\left(\bar{x}_{n} \in U_{n, i}, j c 16^{n^{-\frac{1}{2}}}\right. & <\frac{\left(\tilde{\theta}_{n, i}-\theta_{0}\right) \cdot\left(\bar{x}_{n}-\lambda\left(\tilde{\theta}_{n, i}\right)\right) n^{\frac{3}{2}}}{\left\{\left(\tilde{\theta}_{n, i}-\theta_{0}\right) \cdot \Sigma_{\tilde{\theta}_{n, i}}\left(\tilde{\theta}_{n, i}-\theta_{0}\right)\right\}^{\frac{3}{2}}} \leq \\
& \left.\leq(j+1) c_{16^{n}}{ }^{-\frac{1}{2}}\right) \geq
\end{aligned}
$$




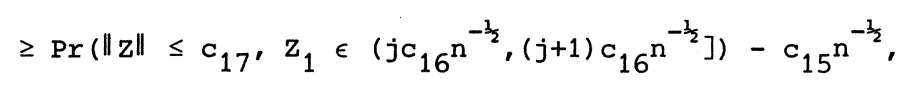

where the k-vector $\mathrm{z}$ has a normal $\mathrm{N}\left(0 ; \mathrm{I}_{k}\right)$ distribution, and $j=0,1, \ldots,\left[\mathrm{n}^{\frac{1}{2}}\right]=$ entier $\left(n^{\frac{1}{2}}\right)$. Note that we can take $c_{15}$ independent of $\tilde{\theta}_{n, i}$ since the third order moments are bounded. Taking the constant $c_{16}$ large enough we find

$$
\begin{aligned}
& P_{\tilde{\theta}_{n, i}}\left(\bar{x}_{n} \in U_{n, i}, j c_{16^{n}}{ }^{-\frac{1}{2}}<\frac{\left(\tilde{\theta}_{n, i}-\theta_{0}\right) \cdot\left(\bar{x}_{n}-\lambda\left(\tilde{\theta}_{n, i}\right)\right) n^{\frac{1}{2}}}{\left\{\left(\tilde{\theta}_{n, i}-\theta_{0}\right) \cdot \Sigma_{\tilde{\theta}_{n, i}}\left(\tilde{\theta}_{n, i}-\theta_{0}\right)\right\}^{\frac{13}{2}}} \leq(j+1) c_{16^{n}}{ }^{-\frac{1}{2}}\right) \\
& \geq c_{18^{n}}{ }^{-\frac{1}{2}} \quad \text { for } j=0,1, \ldots\left[n^{\frac{1}{2}}\right] .
\end{aligned}
$$

It follows that (cf. (3.2.8))

$$
\begin{aligned}
& \text { (3.2.9) } \int_{\left\{U_{n, i},\left(\tilde{\theta}_{n, i}-\theta_{0}\right) ' x \geq\left(\tilde{\theta}_{n, i}-\theta_{0}\right) \cdot \lambda\left(\tilde{\theta}_{n, i}\right)\right\}} \exp \left\{-n\left(\tilde{\theta}_{n}-\theta_{0}\right) \cdot\left(x-\lambda\left(\tilde{\theta}_{n, i}\right)\right)\right\} d \bar{P}_{\tilde{\theta}_{n, i}^{n}}^{n}(x) \\
& \left.\geq \sum_{j=0}^{\left[n^{\frac{1}{2}}\right]} \int \quad \exp \left\{-n\left(\tilde{\theta}_{n, i}-\theta_{0}\right)\right)^{\prime}\left(x-\lambda\left(\tilde{\theta}_{n, i}\right)\right)\right\} d \bar{P}_{\tilde{\theta}_{n, i}}^{n}(x) \\
& \left\{U_{n, i}, \frac{\left(\tilde{\theta}_{n, i}-\theta_{0}\right) \cdot\left(x-\lambda\left(\tilde{\theta}_{n, i}\right) n^{\frac{1}{2}}\right.}{\left\{\left(\tilde{\theta}_{n, i}-\theta_{0}\right) \cdot \Sigma_{\tilde{\theta}_{n, i}}\left(\tilde{\theta}_{n, i}-\theta_{0}\right)\right\}^{\frac{1}{2}}} \in\left(j c_{16} n^{-\frac{1}{2}},(j+1) c_{16^{n}}{ }^{-\frac{1}{2}}\right]\right\} \\
& \geq \sum_{j=0}^{\left[n^{\frac{1}{2}}\right]} c_{18^{n}} n^{-\frac{1}{2}} \exp \left\{-(j+1) c_{16}\left\{\left(\tilde{\theta}_{n, i}-\theta_{0}\right) \cdot \sum_{\tilde{\theta}_{n, i}}\left(\tilde{\theta}_{n, i}-\theta_{0}\right)\right\}^{\frac{3}{2}}\right\} \\
& \geq \sum_{j=0}^{\left[n^{\frac{1}{2}}\right]} c_{18^{n}} n^{-\frac{1}{2}} \exp \left\{-(j+1) c_{19} d_{n}^{\frac{3}{2}}\right\} \\
& \geq c_{18} n^{-\frac{1}{2}} \exp \left(-c_{19} d_{n}^{\frac{1}{2}}\right) \frac{1-\exp \left(-c_{19} d^{\frac{1}{2}}\left[n^{\frac{3}{2}}\right]\right)}{1-\exp \left(-c_{19} d_{n}^{\frac{1}{2}}\right)} \\
& \geq c_{20}\left(n d_{n}\right)^{-\frac{1}{2}},
\end{aligned}
$$

where the third inequality follows by

$$
\left(\tilde{\theta}_{n, i}-\theta_{0}\right)^{\prime} \Sigma_{\tilde{\theta}_{n, i}}\left(\tilde{\theta}_{n, i}-\theta_{0}\right) \leq\left\|\tilde{\theta}_{n, i}-\theta_{0}\right\|^{2} \sup _{\substack{\|u\|^{\prime}=1 \\ \zeta \in K_{0}}} u^{\prime} \Sigma_{\zeta} u \leq c_{16}^{-2} c_{19}^{2} d_{n} .
$$

Combining (3.2.8) and (3.2.9) we find (cf. (3.2.7) and (3.2.4)) 


$$
\begin{aligned}
P_{\theta_{0}}\left(\bar{x}_{n} \notin \lambda\left\{\theta ; I\left(\theta, \theta_{0}\right) \leq d_{n}\right\}\right) & \geq \sum_{i=1}^{p_{n}} c_{20}\left(n d_{n}\right)^{-\frac{1}{2}} \exp \left(-n d_{n}\right) \\
& \geq c_{5} c_{20}\left(n d_{n}\right)^{\frac{k-2}{2}} \exp \left(-n d_{n}\right) .
\end{aligned}
$$

So we have established (3.2.6) with $c_{13}=c_{5} c_{20}$.

It remains to prove that

$$
P_{\theta_{0}}\left(\dot{\mathrm{x}}_{\mathrm{n}} \notin \lambda\left\{\theta_{i} I\left(\theta, \theta_{0}\right)<\mathrm{d}_{\mathrm{n}}\right\}\right) \leq \mathrm{c}_{21}\left(\mathrm{nd} \mathrm{d}_{\mathrm{n}}\right)^{\frac{\mathrm{k}-2}{2}} \exp \left(-\mathrm{nd} \mathrm{d}_{\mathrm{n}}\right) \text {. }
$$

To this end we first prove the following statement: if $x \notin \lambda\left\{\theta_{i} I\left(\theta, \theta_{0}\right)<d_{n}\right\}$ then there is a $\tilde{\theta}_{n, i}\left(1 \leq i \leq p_{n}\right)$ such that

$$
\left(\tilde{\theta}_{n, i}-\theta_{0}\right) \cdot x-\psi\left(\tilde{\theta}_{n, i}\right)+\psi\left(\theta_{0}\right) \geq d_{n}-c_{22} n^{-1}
$$

Geometrically: we cover the region outside $\left\{\lambda(\theta) ; I\left(\theta, \theta_{0}\right) \leq d_{n}\right\}$ by (suitable chosen) halfspaces.

To show this we distinguish two cases.

(i) $x=\lambda(\theta)$ for some $\theta$ satisfying $I\left(\theta, \theta_{0}\right)=d_{n}$. Then there is a $\tilde{\theta}_{n, i}$ $\left(1 \leq i \leq p_{n}\right)$ such that $\left\|\theta-\tilde{\theta}_{n, i}\right\| \leq c_{12^{n}}{ }^{-\frac{1}{2}}$, and thus (by lemma $3.2 .2(i i)$ )

$$
\begin{aligned}
\left(\tilde{\theta}_{n, i}-\theta_{0}\right)^{\prime} x-\psi\left(\tilde{\theta}_{n, i}\right)+\psi\left(\theta_{0}\right) & =\left(\tilde{\theta}_{n, i}-\theta_{0}\right) \cdot \lambda(\theta)-\psi\left(\tilde{\theta}_{n, i}\right)+\psi\left(\theta_{0}\right)= \\
& =I\left(\theta_{,} \theta_{0}\right)-I\left(\theta, \tilde{\theta}_{n, i}\right) \geq d_{n}-c_{22} n^{-1} .
\end{aligned}
$$

Hence (3.2.11) is satisfied.

(ii) $x=\lambda(\theta)+\gamma\left\{\lambda(\theta)-\lambda\left(\theta_{0}\right)\right\}$ with $I\left(\theta, \theta_{0}\right)=d_{n}$ and $\gamma>0$.

Again there exists a $\tilde{\theta}_{n, i}\left(1 \leq i \leq p_{n}\right)$ such that $\left\|\tilde{\theta}_{n, i}-\theta\right\| \leq c_{12} n^{-\frac{1}{2}}$, which implies $\left\|\lambda\left(\tilde{\theta}_{n, i}\right)-\lambda(\theta)\right\|^{n, i} c_{23} n^{-\frac{1}{2}}$. Since in this case

$$
\begin{aligned}
& \left(\tilde{\theta}_{n, i}-\theta_{0}\right) \cdot x-\psi\left(\tilde{\theta}_{n, i}\right)+\psi\left(\theta_{0}\right)= \\
& =\left(\tilde{\theta}_{n, i}-\theta_{0}\right) \cdot \lambda(\theta)-\psi\left(\tilde{\theta}_{n, i}\right)+\psi\left(\theta_{0}\right)+\gamma\left(\tilde{\theta}_{n, i}-\theta_{0}\right) \cdot\left\{\lambda(\theta)-\lambda\left(\theta_{0}\right)\right\},
\end{aligned}
$$

and $\left(\tilde{\theta}_{n, i}-\theta_{0}\right) \cdot \lambda(\theta)-\psi\left(\tilde{\theta}_{n, i}\right)+\psi\left(\theta_{0}\right) \geq a_{n}-c_{22} n^{-1}$ by (i), it is sufficient to prove that $\left(\tilde{\theta}_{n, i}-\theta_{0}\right) \cdot\left\{\lambda(\theta)-\lambda\left(\theta_{0}\right)\right\} \geq 0$. Now by an application of lemma 3.2 .2 


$$
\begin{aligned}
& \left(\tilde{\theta}_{n, i}-\theta_{0}\right) \cdot\left\{\lambda(\theta)-\lambda\left(\theta_{0}\right)\right\}= \\
& =\left(\tilde{\theta}_{n, i}-\theta_{0}\right) \cdot\left\{\lambda\left(\tilde{\theta}_{n, i}\right)-\lambda\left(\theta_{0}\right)\right\}+\left(\tilde{\theta}_{n, i}-\theta_{0}\right) \cdot\left\{\lambda(\theta)-\lambda\left(\tilde{\theta}_{n, i}\right)\right\} \\
& \geq c_{24} d_{n}-c_{6}^{\frac{1}{2}} d_{n}^{\frac{1}{2}} c_{23} n^{-\frac{1}{2}} \geq 0
\end{aligned}
$$

for sufficiently large $n$, since $n d_{n} \rightarrow \infty$. This completes the proof of $(3.2 .11)$. It follows that

$$
\begin{aligned}
& P_{\theta_{0}}\left(\bar{x}_{n} \notin \lambda\left\{\theta ; I\left(\theta, \theta_{0}\right)<a_{n}\right\}\right) \leq \\
& \leq \sum_{i=1}^{p_{n}} P_{\theta_{0}}\left(\left(\tilde{\theta}_{n, i}-\theta_{0}\right) \cdot \bar{x}_{n}-\psi\left(\tilde{\theta}_{n, i}\right)+\psi\left(\theta_{0}\right) \geq a_{n}-c_{22} n^{-1}\right) .
\end{aligned}
$$

Again we consider one term of this sum:

$$
\begin{aligned}
& \text { (3.2.13) } P_{\theta_{0}}\left(\left(\tilde{\theta}_{n, i}-\theta_{0}\right) \cdot \bar{x}_{n}-\psi\left(\tilde{\theta}_{n, i}\right)+\psi\left(\theta_{0}\right) \geq d_{n}-c_{22} n^{-1}\right)= \\
& =P_{\theta_{0}}\left(\left(\tilde{\theta}_{n, i}-\theta_{0}\right) \cdot\left(\bar{x}_{n}-\lambda\left(\tilde{\theta}_{n, i}\right)\right) \geq-c_{22} n^{-1}\right) \\
& \leq \sum_{j=0}^{\infty} \int \quad \exp \left\{n\left(\theta_{0}-\tilde{\theta}_{n, i}\right) \cdot x-n \psi\left(\theta_{0}\right)+n \psi\left(\tilde{\theta}_{n, i}\right)\right\} d \bar{P}_{\tilde{\theta}}^{n} \quad(x) \\
& \left\{j n^{-\frac{1}{2}} \leq \frac{\left(\tilde{\theta}_{n, i}-\theta_{0}\right) \cdot\left(x-\lambda\left(\tilde{\theta}_{n, i}\right)\right) n^{\frac{1}{2}}+c_{22^{n}} n^{-\frac{1}{2}}}{\left\{\left(\tilde{\theta}_{n, i}-\theta_{0}\right) \cdot \Sigma_{\tilde{\theta}_{n, i}}\left(\tilde{\theta}_{n, i}-\theta_{0}\right)\right\}^{\frac{1}{2}}} \leq(j+1) n^{-\frac{1}{2}}\right\} \\
& \leq \exp \left(-n d_{n}\right) \sum_{j=0}^{\infty} \exp \left\{c_{22}-j\left\{\left(\tilde{\theta}_{n, i}-\theta_{0}\right) \cdot \Sigma_{\tilde{\theta}_{n, i}}\left(\tilde{\theta}_{n, i}-\theta_{0}\right)\right\}^{\frac{1}{2}}\right\} \times
\end{aligned}
$$

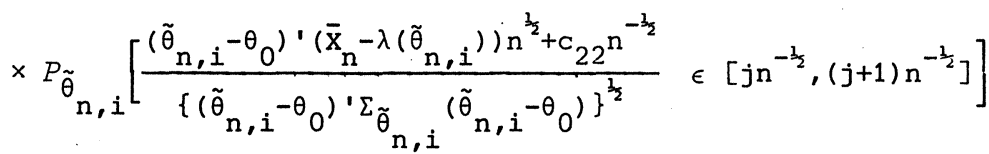

$$
\begin{aligned}
& \leq \exp \left(-n d_{n}\right) \sum_{j=0}^{\infty} c_{25^{n}} n^{-\frac{1}{2}} \exp \left(c_{22}-j c_{26} d_{n}^{\frac{3}{2}}\right) \\
& \leq c_{27}\left(n d_{n}\right)^{-\frac{1}{2}} \exp \left(-n d_{n}\right) \text {, }
\end{aligned}
$$

where the third inequality is a consequence of the one-dimensional BerryEsseen theorem. Hence 


$$
\begin{aligned}
& P_{\theta_{0}}\left(\bar{x}_{n} \notin \lambda\left\{\theta ; I\left(\theta, \theta_{0}\right)<d_{n}\right\}\right) \leq \\
& \leq \sum_{i=1}^{p_{n}} c_{27}\left(n d_{n}\right)^{-\frac{1}{2}} \exp \left(-n d_{n}\right) \\
& \leq c_{4} c_{27}\left(n d_{n}\right)^{\frac{k-2}{2}} \exp \left(-n d_{n}\right),
\end{aligned}
$$

proving $(3 \cdot 2 \cdot 10)$.

The constants $c_{1}, \ldots, c_{27}$ appearing in the proof can be chosen independent of $\theta_{0}$; for instance $c_{1}=\inf \left\{\|\theta-\xi\|^{2}\{I(\theta, \xi)\}^{-1} ; \theta, \xi \in K_{0}\right\}$. Hence (3.2.2) holds uniformly for $\theta_{0} \in \mathrm{K}$.

This completes the proof of the theorem.

3.3. THE NULL HYPOTHESIS CONTAINED IN A COMPACT SUBSET OF INT $\theta$

We start with a useful lemma.

LEMMA 3.3.1. If $\theta_{0}$ and $\theta_{1}$ are such that int $\theta_{1} \neq \varnothing$ and $\operatorname{cl} \theta_{0} \wedge$ int $\theta \neq \varnothing$, then $\lim _{\mathrm{n} \rightarrow \infty} \alpha_{\mathrm{n}}=0$ implies $\lim _{\mathrm{n} \rightarrow \infty} \mathrm{nd} \mathrm{n}_{\mathrm{n}}=\infty$.

PROOF. Suppose lim inf ${ }_{n \rightarrow \infty} n d_{n}<\infty$. Assume without loss of generality that nd ${ }_{n} \leq C$ for all $\mathrm{n}(C>0)$. Let $\theta_{0} \in \mathrm{cl} \theta_{0} \wedge$ int $\theta$ and $B_{\varepsilon}$ be an open sphere with radius $\varepsilon>0$ such that $B_{\varepsilon} \subset$ int $\theta_{1}$ and $\theta_{0}$ is a boundary point of $B_{\varepsilon}$. Then it can be shown that $\alpha_{n} \geq E_{\theta_{0}} \phi_{n}^{L R}\left(\bar{x}_{n}\right) \geq P_{\theta_{0}}\left(I\left(\lambda^{-1}\left(\bar{x}_{n}\right), \theta_{0}\right)>\mathrm{Cn}^{-1} \wedge\right.$ $\left.\bar{x}_{n} \in \lambda\left(B_{\varepsilon}\right)\right) \geq \delta>0$ for sufficiently large $n$, in contradiction to $\lim _{n \rightarrow \infty} \alpha_{n}=0$.

We consider a null hypothesis $\theta_{0}$ contained in a compact set $\mathrm{K} \subset$ int $\theta$ (the easiest situation, that of a simple null hypothesis, is a special case).

In chapter II we encountered such a null hypothesis in corollary 2.6.1 to theorem 2.5.1. Although we have no generalization of theorem 2.5.1 (see section 3.1 ), the statement of corollary 2.6 .1 remains true:

THEOREM 3.3.2. If $\theta_{0} \subset \mathrm{K}$, where $\mathrm{K}$ is a compact subset of int $\theta$, and if $I<I\left(\Theta_{0}\right)$ (cf. $\left.(3.2 .1)\right)$ exists such that

$$
\text { (3.3.1) } \alpha_{n} \geq \exp (-n I) \text { for all sufficiently large } n \text {, }
$$

then $\lim _{n \rightarrow \infty} R_{n}(\theta)=0$ uniformly on $\theta_{1}$. 
As ever our first step is to connect $\alpha_{n}$ and $\alpha_{n}$. With the help of theorem 3.2.1 we derive an upper bound for $\alpha_{n}$ in terms of $d_{n}$ :

LEMMA 3.3.3. If (3.3.1) is satisfied, then there exists a positive constant C such that

$(3.3 .2) \quad \alpha_{n} \leq c\left(n d_{n}\right)^{\frac{k-2}{2}} \exp \left(-n d_{n}\right)$.

PROOF. Since

$$
\begin{aligned}
\alpha_{n} & \leq \sup _{\theta_{0} \in \Theta_{0}} P_{\theta_{0}}\left(\sup _{\vartheta \in \Theta_{0}}\left\{\vartheta \cdot \bar{x}_{n}-\psi(\vartheta)\right\}-\sup _{\theta \in \Theta}\left\{\theta \cdot \bar{x}_{n}-\psi(\theta)\right\} \leq-d_{n}\right) \\
& \leq \sup _{\theta_{0} \in \Theta_{0}} P_{\theta_{0}}\left(\theta_{0}^{\prime} \bar{x}_{n}-\psi\left(\theta_{0}\right)-\sup _{\theta \in \Theta}\left\{\theta^{\prime} \bar{x}_{n}-\psi(\theta)\right\} \leq-d_{n}\right) \\
& \leq \sup _{\theta_{0} \in \theta_{0}} P_{\theta_{0}}\left(\bar{x}_{n} \notin \lambda\left\{\theta ; I\left(\theta, \theta_{0}\right)<d_{n}\right\}\right),
\end{aligned}
$$

we are almost in the situation where theorem 3.2 .1 can be applied. Let $0<\delta<I\left(\theta_{0}\right)-I$ then by theorem 3.2 .1 there exists $c_{\delta}>0$ such that

$$
\begin{aligned}
& \sup _{\theta_{0} \in \Theta_{0}} P_{\theta_{0}}\left(\bar{X}_{n} \notin \lambda\left\{\theta ; I\left(\theta, \theta_{0}\right) \leq I+\frac{1}{2} \delta\right\}\right) \\
& \leq c_{\delta}\left[n\left(I+\frac{1}{2} \delta\right)\right]^{\frac{k-2}{2}} \exp \left\{-n\left(I+\frac{1}{2} \delta\right)\right\} .
\end{aligned}
$$

Now suppose that $\lim \sup _{n \rightarrow \infty} d_{n}>I$, then there is a subsequence $\left\{d_{n_{i}}\right\}$ with $\lim _{i \rightarrow \infty} d_{n_{i}} \geq I+\delta_{0}$ for some $0<\delta_{0}<I\left(\theta_{0}\right)-I$. Then for sufficiently large $i$ is

$$
\begin{aligned}
& \alpha_{n_{i}} \leq \sup _{\theta_{0} \in \theta_{0}} P_{\theta_{0}}\left(\bar{x}_{n_{i}} \notin \lambda\left\{\theta ; I\left(\theta, \theta_{0}\right)<\alpha_{n_{i}}\right\}\right) \\
& \leq \sup _{\theta_{0} \in \Theta_{0}} P_{\theta_{0}}\left(\bar{x}_{n_{i}} \notin \lambda\left\{\theta ; I\left(\theta, \theta_{0}\right)<I+\frac{1}{2} \delta_{0}\right\}\right) \\
& \leq c_{\delta_{0}}\left[n_{i}\left(I+\frac{1}{2} \delta_{0}\right)\right]^{\frac{k-2}{2}} \exp \left\{-n_{i}\left(I+\frac{1}{2} \delta_{0}\right)\right\} \text {, }
\end{aligned}
$$

in contradiction to $(3 \cdot 3 \cdot 1)$. Hence $\lim \sup _{n \rightarrow \infty} d_{n} \leq I$. 
On the other hand lemma 3.3.1 implies that $\mathrm{nd}_{\mathrm{n}} \rightarrow \infty$ and thus $a_{n} \geq \varepsilon n^{-1}$ for sufficiently large $n$.

Application of theorem 3.2.1 yields

$$
\begin{aligned}
\alpha_{n} & \leq \sup _{\theta_{0} \in \Theta_{0}} P_{\theta_{0}}\left(\bar{x}_{n} \notin \lambda\left\{\theta ; I\left(\theta, \theta_{0}\right)<d_{n}\right\}\right) \\
& \leq c_{\delta_{0}}\left(n d_{n}\right)^{\frac{k-2}{2}} \exp \left(-n d_{n}\right)
\end{aligned}
$$

as was to be proved.

PROOF OF THEOREM 3.3.2. Let $c_{1}, \ldots, c_{12}$ be appropriate positive constants. We shall prove that for every sequence $\left\{\theta_{n}\right\}$ satisfying $I\left(\theta_{n}, \theta_{0}\right) \leq I+\delta$ $\left(0<\delta<I\left(\theta_{0}\right)-I\right)$ and $R_{n}\left(\theta_{n}\right) \geq \varepsilon>0$ it holds that $\alpha_{n} \geq c_{1} \exp \left\{-n d_{n}+c_{2}\left(n d_{n}\right)^{\frac{1}{2}}\right\}$. Since by lemma $3.3 .1 \mathrm{nd}_{\mathrm{n}} \rightarrow \infty$, a contradiction to lemma 3.3.3 is obtained for these sequences $\left\{\theta_{n}\right\}$. We shall also show that $\lim _{n \rightarrow \infty} E_{\theta_{n}} \phi_{n}^{L R}\left(\bar{X}_{n}\right)=1$ for every sequence $\left\{\theta_{n}\right\}$ satisfying $I\left(\theta_{n}, \theta_{0}\right)>I+\delta$. Together these two results yield the theorem.

Part a.

Consider a sequence $\left\{\theta_{n}\right\}$ in $\theta$ such that $I\left(\theta_{n}, \theta_{0}\right) \leq I+\delta$ and $R_{n}\left(\theta_{n}\right) \geq \varepsilon>0$. Assume without loss of generality $\theta_{n} \notin$ cl $\theta_{0}$. For sufficiently large $n$ there exists a sphere $B_{n} \subset \lambda($ int $\theta)$ with centre $\lambda\left(\theta_{n}\right)$ and radius $c_{3} n^{-\frac{1}{2}}$ such that $P_{\theta_{n}}\left(\bar{x}_{n} \in B_{n}\right) \geq 1-\frac{1}{2} \varepsilon$, for

$$
\Sigma_{\theta_{n}}^{-\frac{1}{2}}\left(\bar{x}_{n}-\lambda\left(\theta_{n}\right)\right) n^{\frac{1}{2}} \rightarrow N\left(0 ; I_{k}\right) \text { under } \theta_{n}
$$

since $\theta_{n}$ lies in a compact subset of int $\theta$. From $R_{n}\left(\theta_{n}\right) \geq \varepsilon$ it follows that

$$
\int_{B_{n}}\left\{\phi_{n}^{+}(x)-\phi_{n}^{L R}(x)\right\} d \bar{P}_{\theta_{n}}^{-n}(x) \geq \frac{1}{2} \varepsilon
$$

and hence

$$
\text { (3.3.4) } \quad \int_{B_{n} \wedge I\left(\lambda^{-1}(x), \theta_{0}\right) \leq d_{n}} \phi_{n}^{+}(x) d \bar{P}_{\theta_{n}^{-n}}^{n}(x) \geq \frac{1}{2} \varepsilon .
$$

Note that $\phi_{\mathrm{n}}^{+}$satisfies 


$$
\phi_{n}^{+}(x)=\left\{\begin{array}{lll}
1 & & < \\
& \text { if } \int_{c l} \exp \left\{n\left(\theta_{0}-\theta_{n}\right) ' x-n \psi\left(\theta_{0}\right)+n \psi\left(\theta_{n}\right)\right\} d \tau_{n}\left(\theta_{0}\right)>n^{\prime} &
\end{array}\right.
$$

where the distribution $\tau_{\mathrm{n}}$ (concentrated on $\mathrm{cl} \theta_{0}$ ) is least favorable (see [15] section 3.8). Define

$$
\begin{aligned}
& t_{n}\left(\theta_{0}, x\right)=\exp \left\{n\left(\theta_{0}-\theta_{n}\right) \cdot x-n \psi\left(\theta_{0}\right)+n \psi\left(\theta_{n}\right)\right\}, \\
& U_{1, n}=\left\{x ; x \in B_{n}, \int_{c l} \theta_{0} t_{n}\left(\theta_{0}, x\right) d \tau_{n}\left(\theta_{0}\right)<t_{n}\right\}
\end{aligned}
$$

and

$$
\mathrm{U}_{2, \mathrm{n}} \quad=\left\{\mathrm{x} ; \mathrm{x} \in \mathrm{B}_{\mathrm{n}}, \int_{\mathrm{Cl} \theta_{0}} \mathrm{t}_{\mathrm{n}}\left(\theta_{0}, \mathrm{x}\right) \mathrm{d} \tau_{\mathrm{n}}\left(\theta_{0}\right) \leq \mathrm{t}_{\mathrm{n}}\right\} .
$$

Then $U_{1, n} \subset\left\{x ; x \in B_{n}, \phi_{n}^{+}(x)=1\right\} \subset U_{2, n}$. We first prove that $\operatorname{int}\left(U_{2, n}-U_{1, n}\right)=\varnothing$. Suppose to the contrary that $\operatorname{int}\left(\mathrm{U}_{2, \mathrm{n}}-\mathrm{U}_{1, \mathrm{n}}\right) \neq \varnothing$. Then there exist, for any fixed $n, x, y_{1}, \ldots, y_{k}$ in $U_{2, n}-U_{1, n}$ with the property that $x-y_{1}, \ldots, x-y_{k}$ are linear independent and $\frac{1}{2} x+\frac{1}{2} y_{i} \in U_{2, n}-U_{1, n}(i=1, \ldots, k)$. Denote by $\mathrm{T}_{\mathrm{y}_{i}}=\left\{\theta_{0} \in \operatorname{cl} \theta_{0} ;\left(\theta_{0}-\theta_{n}\right) \cdot \mathrm{x}=\left(\theta_{0}-\theta_{n}\right)^{\prime} \mathrm{y}_{i}\right\}$. Then

$$
\begin{aligned}
0 & =\int_{\operatorname{cl} \theta_{0}}\left\{\frac{1}{2} t_{n}\left(\theta_{0}, x\right)+\frac{1}{2} t_{n}\left(\theta_{0}, y_{i}\right)-t_{n}\left(\theta_{0}, \frac{1}{2} x+\frac{1}{2} y_{i}\right)\right\} d \tau_{n}\left(\theta_{0}\right) \\
& =\int_{T_{Y_{i}}^{C}}\left\{\frac{1}{2} t_{n}\left(\theta_{0}, x\right)+\frac{1}{2} t_{n}\left(\theta_{0}, y_{i}\right)-t_{n}\left(\theta_{0}, \frac{1}{2} x+\frac{1}{2} y_{i}\right)\right\} d \tau_{n}\left(\theta_{0}\right) .
\end{aligned}
$$

The last equality is a consequence of the fact that the integrand is non-negative due to the convexity of $t_{n}\left(\theta_{0}, \cdot\right)$. Since the integrand is positive on $T_{Y_{i}}^{c}$, it follows that $\tau_{n}\left(T_{Y_{i}}^{c}\right)=0(i=1, \ldots, k)$, and hence $\tau_{n}\left(U_{i=1}^{k} T_{Y_{i}}^{C}\right)=0$. The linear independence of $x-y_{1}, \ldots, x-y_{k}$ implies that $U_{i=1}^{k} T_{Y_{i}}^{c} \wedge c l \theta_{0}=c l \theta_{0} \backslash \theta_{n}=c l \theta_{0}$, because $\theta_{n} \notin c l \theta_{0}$, and thus $\tau_{n}\left(c l \theta_{0}\right)=0$, in contradiction to the definition of $\tau_{n}$, which proves that $\operatorname{int}\left(\mathrm{U}_{2, \mathrm{n}}-\mathrm{U}_{1, \mathrm{n}}\right)=\varnothing$.

Since the Lebesgue measure of $\mathrm{U}_{2, \mathrm{n}}-\mathrm{U}_{1, \mathrm{n}}$ is zero for all $\mathrm{n}$ and (3.3.3) holds the probability of randomization of the MP test vanishes under $\theta_{n}$ as $n \rightarrow \infty$. Together with (3.3.4) it follows that

$$
P_{\theta_{\mathrm{n}}}\left(\overline{\mathrm{x}}_{\mathrm{n}} \in \mathrm{U}_{1, \mathrm{n}^{\prime}} \mathrm{I}\left(\lambda^{-1}\left(\overline{\mathrm{x}}_{\mathrm{n}}\right), \theta_{0}\right) \leq \mathrm{d}_{\mathrm{n}}\right) \geq \varepsilon / 4
$$

for sufficiently large $n$. We further note that $U_{1, n}$ is a convex set. 
We now claim that for $\mathrm{n} \geq \mathrm{n}_{0}$ there exists a point $\zeta_{\mathrm{n}} \in \theta$ with the following properties:

$$
\begin{aligned}
& \lambda\left(\zeta_{n}\right) \in B_{n} \wedge\left\{x ; \phi_{n}^{+}(x)=1\right\}, \\
& I\left(\zeta_{n}, \theta_{0}\right) \leq d_{n} \text {, and }
\end{aligned}
$$

there exists a sphere $B_{n}^{*}$ with centre $\lambda\left(\zeta_{n}\right)$ and radius $C_{4} n^{-\frac{1}{2}}$

contained in $B_{n} \wedge\left\{x ; \phi_{n}^{+}(x)=1\right\}$.

For: (3.3.3), (3.3.5) and the inequality $\left\|\Sigma_{\theta_{n}}^{-\frac{1}{2}} x-\Sigma_{\theta_{n}}^{-\frac{1}{2}} y\right\| \leq c_{5}{ }^{c} 4^{n^{-\frac{1}{2}}}$ for all $x, y$ satisfying $\|x-y\| \leq c_{4} n^{-\frac{1}{2}}$ imply that such points $\zeta_{n}$ exist, provided $c_{4}$ is sufficiently small.

Since $I\left(\zeta_{n}, \theta_{0}\right) \leq d_{n}$ there exists a point $\xi_{n} \in \theta_{0}$ with $I\left(\zeta_{n}, \xi_{n}\right) \leq$ $d_{n}+n^{-1}$. Let $n_{n} \in \theta$ satisfy (for some positive constants $c_{1}$ and $c_{2}$ )

(i) $\lambda\left(n_{n}\right) \in B_{n^{\prime}}$

(ii) $I\left(n_{n}, \xi_{n}\right) \leq d_{n}-c 2^{n^{-\frac{3}{2}}} d_{n}^{\frac{3}{2}}$, and

(iii) $P_{n_{n}}\left(\bar{x}_{n} \in B_{n^{\prime}}^{*}\left(\xi_{n}-n_{n}\right) \cdot \bar{x}_{n}>\left(\xi_{n}-n_{n}\right) \cdot \lambda\left(n_{n}\right)\right) \geq c_{1}$ for all $n \geq n_{1}$.

The existence of such points $\eta_{n}$ (and $c_{1}$ and $c_{2}$ ) may be argued as follows: Suppose that $I\left(\zeta_{n}, \xi_{n}\right) \leq d_{n}-d_{n}^{\frac{1}{2} n^{-\frac{1}{2}}}$; taking $\eta_{n}=\zeta_{n}$ all the required properties of $\eta_{n}$ are satisfied.

If $I\left(\zeta_{n}, \xi_{n}\right)>d_{n}-d_{n}^{\frac{1}{2}} n^{-\frac{1}{2}}$ the proof is more difficult. Choose $\eta_{n}$ on the line through $\zeta_{n}$ and $\xi_{n}: n_{n}=\zeta_{n}+\gamma_{n}\left(\xi_{n}-\zeta_{n}\right) \quad\left(0<\gamma_{n}<1\right)$ such that $\left\|\lambda\left(n_{n}\right)-\lambda\left(\zeta_{n}\right)\right\|=$ ${ }_{\frac{1}{2} \mathrm{C}} \mathrm{n}^{-\frac{1}{2}}$. This is possible if $\left\|\lambda\left(\zeta_{n}\right)-\lambda\left(\xi_{n}\right)\right\|>3_{2}^{\frac{1}{2} c} n^{-\frac{1}{2}}$, but by lemma 3.2 .2 $\left\|\lambda\left(\zeta_{n}\right)-\lambda\left(\xi_{n}\right)\right\| \geq c_{6}\left[I\left(\zeta_{n}, \xi_{n}\right)\right]^{\frac{1}{2}} \geq c_{6}\left(d_{n}-d_{n}^{\frac{1}{2}} n^{-\frac{1}{2}}\right)^{\frac{1}{2}} \geq c_{6}\left(\frac{1}{4} d_{n}\right)^{\frac{1}{2}}>\frac{1}{2} c_{4} n^{-\frac{1}{2}}$ since $n d_{n} \rightarrow \infty$. Thus $n_{n}$ is well defined and obviously satisfies (i) and (iii). It remains to prove (ii).

Since $I\left(n_{n}, \xi_{n}\right)=I\left(\zeta_{n}, \xi_{n}\right)+I\left(n_{n}, \zeta_{n}\right)-\left(\xi_{n}-\zeta_{n}\right) \cdot\left(\lambda\left(n_{n}\right)-\lambda\left(\zeta_{n}\right)\right)$, and by lemma 3.2 .2

$$
\begin{aligned}
& \frac{\left(\xi_{n}-\zeta_{n}\right)^{\prime}\left(\lambda\left(n_{n}\right)-\lambda\left(\zeta_{n}\right)\right)}{\left\|\xi_{n}-\zeta_{n}\right\|\left\|n_{n}-\zeta_{n}\right\|}=\frac{\left(n_{n}-\zeta_{n}\right)^{\prime}\left(\lambda\left(n_{n}\right)-\lambda\left(\zeta_{n}\right)\right)}{\left\|n_{n}-\zeta_{n}\right\|^{2}} \geq c_{7} \\
& \left\|\xi_{n}-\zeta_{n}\right\| \geq c_{8} d_{n}^{\frac{3}{2}}
\end{aligned}
$$

and

$$
\left\|n_{n}-\zeta_{n}\right\| \geq c_{9} n^{-\frac{1}{2}},
$$

it follows that 


$$
I\left(n_{n}, \xi_{n}\right) \leq d_{n}+n^{-1}+c_{10} n^{-1}-c_{7} c_{8} c 9^{n^{-\frac{1}{2}}} d_{n}^{\frac{13}{2}} \leq d_{n}-c_{2} n^{-\frac{1}{2}} d_{n}^{\frac{1}{2}}
$$

because $n d_{n} \rightarrow \infty$. Hence points $n_{n}$ satisfying (i), (ii) and (iii) do indeed exist. Consequently

$$
\begin{aligned}
\alpha_{n} \geq & P_{\xi_{n}}\left(\bar{x}_{n} \in\left\{x ; \phi_{n}^{+}(x)=1\right\}\right) \\
\geq & P_{\xi_{n}}\left(\bar{x}_{n} \in B_{n}^{*}\right) \\
\geq & \int_{n}^{*} \wedge\left(\xi_{n}-n_{n}\right)^{\prime} x>\left(\xi_{n}-n_{n}\right) \cdot \lambda\left(n_{n}\right) \\
& \geq c_{1} \exp \left\{n\left(\xi_{n}-n_{n}\right) \cdot \lambda\left(n_{n}\right)-n \psi\left(\xi_{n}\right)+n \psi\left(n_{n}\right)\right\} \\
\geq & c_{1} \exp \left\{-n I\left(n_{n}, \xi_{n}\right)\right\} \\
\geq & c_{1} \exp \left\{-n d_{n}+c_{2}\left(n d_{n}\right)^{\frac{1}{2}}\right\}
\end{aligned}
$$

which completes the proof of part a.

Part b.

Now consider a sequence $\left\{\theta_{n}\right\}$ satisfying $I\left(\theta_{n}, \theta_{0}\right)>I+\delta$. First note that $x \notin \Lambda$ implies $\phi_{n}^{L R}(x)=1$ for sufficiently large $n$. To prove this property let $x \notin \Lambda$. There exists a $\theta_{0}^{*} \in c l \theta_{0}$ such that $\sup _{\theta_{0} \in \Theta_{0}}\left\{\theta_{0}^{\prime} x-\psi\left(\theta_{0}\right)\right\}=$ $\theta_{0}^{*}{ }^{\prime} x-\psi\left(\theta_{0}^{*}\right)$. Choose $\bar{\theta} \in \theta$ such that both $I\left(\bar{\theta}, \theta_{0}\right)>d_{n}$ and $\lambda(\bar{\theta})$ lies on the line segment joining $x$ and $\lambda\left(\theta_{0}^{*}\right): x=\lambda(\bar{\theta})-c_{11}\left\{\lambda\left(\theta_{0}^{*}\right)-\lambda(\bar{\theta})\right\}$ (this is possible for sufficiently large $n$ since $\lim \sup _{n \rightarrow \infty} d_{n} \leq I$ by lemma 3.3.3). Thus

$$
\begin{aligned}
& \sup _{\theta_{0} \in \Theta_{0}}\left\{\theta_{0}^{\prime} x-\psi\left(\theta_{0}\right)\right\}-\sup _{\theta \in \theta}\left\{\theta^{\prime} x-\psi(\theta)\right\} \leq \\
& \leq \theta_{0}^{*}{ }^{\prime} x-\psi\left(\theta_{0}^{*}\right)-\bar{\theta}^{\prime} x+\psi(\bar{\theta}) \\
& =\left(\bar{\theta}-\theta_{0}^{*}\right) \cdot(\lambda(\bar{\theta})-x)-I\left(\bar{\theta}, \theta_{0}^{*}\right) \\
& =c_{11}\left(\bar{\theta}-\theta_{0}^{*}\right) \cdot\left\{\lambda\left(\theta_{0}^{*}\right)-\lambda(\bar{\theta})\right\}-I\left(\bar{\theta}, \theta_{0}^{*}\right) \\
& \leq-I\left(\bar{\theta}, \theta_{0}^{*}\right) \leq-I\left(\bar{\theta}, \theta_{0}\right)<-d_{n}
\end{aligned}
$$


and hence $\phi_{n}^{L R}(x)=1$. It follows that for sufficiently large $n$

$$
\text { (3.3.6) } \quad \begin{aligned}
& P_{\theta_{n}}\left(\bar{x}_{n} \in\left\{x ; \phi_{n}^{L R}(x)<1\right\}\right)= \\
& =P_{\theta_{n}}\left(\bar{x}_{n} \in\left\{x ; \phi_{n}^{L R}(x)<1\right\} \wedge \bar{x}_{n} \in \Lambda\right) \\
& \leq P_{\theta_{n}}\left(I\left(\lambda^{-1}\left(\bar{x}_{n}\right), \theta_{0}\right) \leq d_{n}\right) \\
& \leq P_{\theta_{n}}\left(I\left(\lambda^{-1}\left(\bar{x}_{n}\right), \theta_{0}\right) \leq I+\frac{1}{2} \delta\right) .
\end{aligned}
$$

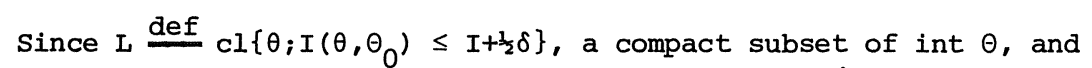
$I\left(\theta_{n}, \theta_{0}\right)>I+\delta$ by assumption, $\inf \left[\left\{I\left(\theta_{n}, L\right) ; n \in \mathbb{N}\right\}, \frac{4 I}{3}\right]=c_{12}>0$. Because $U_{\theta_{0} \in L}\left\{\theta ; I\left(\theta, \theta_{0}\right)<\frac{1}{2} C_{12}\right\}$ is an open cover of $L$ and $L$ is compact, there exist $\theta_{01}, \ldots, \theta_{0 t} \in L$ such that $L \subset U_{i=1}^{t}\left\{\theta ; I\left(\theta, \theta_{0 i}\right)<\frac{1}{2} c_{12}\right\}$. Hence

$$
\begin{aligned}
& P_{\theta_{n}}\left(I\left(\lambda^{-1}\left(\bar{x}_{n}\right), \theta_{0}\right) \leq I+\frac{1}{2} \delta\right) \leq \\
& \leq \sum_{i=1}^{t} P_{\theta_{n}}\left(I\left(\lambda^{-1}\left(\bar{X}_{n}\right), \theta_{0 i}\right)<\frac{1}{2} C_{12}\right) .
\end{aligned}
$$

Consider one term of this sum: $P_{\theta_{n}}\left(I\left(\lambda^{-1}\left(\bar{X}_{n}\right), \theta_{0 i}\right)<\frac{1}{2} c_{12}\right)$. Since $I\left(\theta_{n}, \theta_{0 i}\right) \geq c_{12}$, we can choose $\theta_{n}^{*} \epsilon$ int $\theta$ on the line segment joining $\theta_{C i}$ and $\theta_{n}$ such that $I\left(\theta_{n}^{*}, \theta_{0 i}\right)=3 c_{12} / 4 \leq I$. Then $\left(\theta_{n}-\theta_{n}^{*}\right)=r_{n}\left(\theta_{n}^{*}-\theta_{0 i}\right)$ with $\lim \inf _{n \rightarrow \infty} x_{n}>0$. In combination with $\sup _{\theta \in \theta^{\prime}}\left\{\theta^{\prime} x-\psi(\theta)\right\}=$ $\lambda^{-1}(x)^{\prime} x-\psi\left(\lambda^{-1}(x)\right)$ for $x \in \Lambda$ this implies that

$$
\begin{aligned}
& P_{\theta_{n}}\left(I\left(\lambda^{-1}\left(\bar{x}_{n}\right), \theta_{0 i}\right)<\frac{1}{2 c}_{12}\right) \leq \\
& \leq P_{\theta_{n}}\left(\psi\left(\theta_{0 i}\right)-\theta_{0 i}^{\prime} \bar{x}_{n}+\theta_{n}^{*} \bar{x}_{n}-\psi\left(\theta_{n}^{*}\right)<I\left(\theta_{n}^{*}, \theta_{0 i}\right)-\frac{1}{4} c_{12}\right) \\
& =P_{\theta_{n}}\left(\left(\theta_{n}^{*}-\theta_{0 i}\right){ }^{\prime}\left(\bar{x}_{n}-\lambda\left(\theta_{n}^{*}\right)\right)<-\frac{1}{4} c_{12}\right) \\
& \left.=\int_{\left\{r_{n}\right.}\left(\theta_{n}^{*}-\theta_{0 i}\right)^{\prime}\left(x-\lambda\left(\theta_{n}^{*}\right)\right)<-\frac{1}{4} c_{12}{ }_{n}\right\} \\
& \leq \exp \left\{-\frac{1}{4} c_{12}{ }_{n}^{n} n-n I\left(\theta_{n}^{*}, \theta_{n}\right)\right\} \rightarrow 0,
\end{aligned}
$$

because $\lim$ inf ${ }_{n \rightarrow \infty} r_{n}>0$. Hence in view of $(3.3 .6),(3.3 .7)$ and $(3.3 .8)$ 


$$
P_{\theta_{n}}\left(\bar{x}_{n} \in\left\{x ; \phi_{n}^{L R}(x)<1\right\}\right) \rightarrow 0
$$

and therefore

$$
E_{\theta_{n}} \phi_{n}^{L R}\left(\bar{x}_{n}\right) \rightarrow 1
$$

This completes the proof of the theorem.

3.4. THE ALTERNȦTIVE HYPOTHESIS CONTAINED IN A COMPACT SUBSET OF INT $\theta$

In section 3.3 we have dealt with a null hypothesis $\theta_{0}$ contained in a compact subset of int $\theta$. Conversely it will be assumed in this section that the alternative hypothesis satisfies such a condition. Then we have the following generalization of the one-dimensional result (cf. corollary 2.6.2):

THEOREM 3.4.1. If $\theta_{1} \subset \mathrm{K}$, a compact subset of int $\theta$, then $\lim _{\mathrm{n} \rightarrow \infty} \mathrm{R}_{\mathrm{n}}(\theta)=0$ uniformly on $\theta_{1}$.

The proof is based on the fact that only that part of $\theta_{0}$ is of interest which is near $\theta_{1}$ and hence all relevant arguments are concerned with a compact subset of int $\theta$. We first prove two lemmas.

LEMMA 3.4.2. If $M$ is a compact subset of int $\theta$ and $n d_{n} \geq \varepsilon>0$ for all $n$, then

$$
\begin{aligned}
& P_{\theta_{0}}\left(\bar{x}_{n} \in \lambda(M), I\left(\lambda^{-1}\left(\bar{x}_{n}\right), \theta_{0}\right) \geq d_{n}\right) \leq \\
& \leq c\left(n d_{n}\right)^{\frac{k-2}{2}} \exp \left(-n d_{n}\right),
\end{aligned}
$$

where $0<\mathrm{c}<\infty$ is a constant independent of $\mathrm{n}$ and $\theta_{0}$.

PROOF. Since int $\theta$ is convex, $M$ may also be assumed to be convex. For any $\eta>0$ let $M(\eta)=\left\{\theta ; \inf \left\{\left\|\theta-\theta_{0}\right\|, \theta_{0} \in M\right\} \leq \eta\right\}$. To show that the constant $c$ can be chosen independent of $\theta_{0}$ we shall consider a sequence $\left\{\theta_{0 n}\right\}$ in stead of $\theta_{0}$. In the sequel the constants $c_{1}, \ldots, c_{16}$ will be appropriate positive constants. Choose $c_{1}$ so small that $M\left(c_{1}\right) \subset$ int $\theta$. We consider two cases a) and b):

a) $\theta_{\text {On }} \in M\left(c_{1}\right)$ for all $n$,

b) $\theta_{\text {On }} \notin M\left(c_{1}\right)$ for all $n$. 
In both cases we shall prove (3.4.1). Since we can pick subsequences $\left\{\theta_{0 m}\right\}$ of $\left\{\theta_{0 n}\right\}$ satisfying the assumptions of one of these cases, this proves the theorem.

First assume that $\lim _{n \rightarrow \infty} d_{n}=0$. In case a) an application of theorem 3.2.1 then yields the result. In case b) $\lim _{n \rightarrow \infty} d_{n}=0$ implies that $I\left(\lambda^{-1}(x), \theta_{0 n}\right) \geq d_{n}$ for all $x \in \lambda(M)$ and sufficiently large $n$, and thus

$$
\begin{aligned}
& P_{\theta_{0 n}}\left(\bar{x}_{n} \in \lambda(M), I\left(\lambda^{-1}\left(\bar{x}_{n}\right), \theta_{0 n}\right) \geq d_{n}\right)= \\
& =P_{\theta_{O n}}\left(\bar{x}_{n} \in \lambda(M), I\left(\lambda^{-1}\left(\bar{x}_{n}\right), \theta_{0 n}\right) \geq \underset{\theta \in M}{\inf } I\left(\theta, \theta_{0 n}\right)\right) .
\end{aligned}
$$

Since the right-hand side of (3.4.1) is much smaller if we replace $d_{n}=o(1)$ by $\inf _{\theta \in M} I\left(\theta, \theta_{0 n}\right)$, it suffices to prove (3.4.1) for sequences $\left\{d_{n}\right\}$ satisfying $\lim \inf _{n \rightarrow \infty} d_{n}>0$. We therefore assume $\lim$ inf ${ }_{n \rightarrow \infty} d_{n}>0$.

Our next aim is to prove for all $n \geq n_{0}$ the following property (A): There exist points $\tilde{\theta}_{n, 1}, \ldots, \tilde{\theta}_{n, p_{n}} \in M\left(c_{1}\right)$ such that for any $\theta \in M$ satisfying $I\left(\theta, \theta_{0 n}\right) \geq d_{n}$ there exists a point $\tilde{\theta} \in M\left(c_{1}\right)$ on the line segment joining $\theta$ and $\theta_{0 n}$ with the property that $d_{n} \leq I\left(\tilde{\theta}_{n, i}, \theta_{0 n}\right)=I\left(\tilde{\theta}, \theta_{0 n}\right)$ and $\left\|\tilde{\theta}-\tilde{\theta}_{n, i}\right\| \leq c^{n^{-\frac{1}{2}}}$ for some $i \in\left\{1, \ldots, p_{n}\right\}$, where $p_{n}$ is bounded above by $\left.c_{3}\left(n_{n}\right)(k-1)\right\}_{2}$.

\section{Case a.}

By lemma 3.2.2 $c_{4} \leq I\left(\theta, \theta_{0 n}\right)\left\|\theta-\theta_{0 n}\right\|^{-2} \leq c_{5}$ for all $\theta \neq \theta_{0 n} \in M\left(c_{1}\right)$ and hence $\left\|\theta-\theta_{0 n}\right\|^{2} \geq c_{5}^{-1} d_{n}$ for all $\theta_{0 n} \in M\left(c_{1}\right)$ and all $\theta \in M$ satisfying $I\left(\theta, \theta_{0 n}\right) \geq d_{n}$. Choose points $\theta_{n, 1}, \ldots, \theta_{n, p_{n}}$ on $B_{n} \stackrel{\text { def }}{=}\left\{\theta ;\left\|\theta-\theta_{0 n}\right\|^{2}=c_{5}^{-1} d_{n}\right\}$ such that for all $\theta \in B_{n}$ there exists $a \theta_{n, i}$ with $\left\|\theta-\theta_{n, i}\right\| \leq n^{-\frac{1}{2}}$ and for all $i \neq j\left\|\theta_{n, i}-\theta_{n, j}\right\|>n^{-\frac{1}{2}}$, where $i, j=1, \ldots, p_{n}$. Then $p_{n}$ is bounded above by $c_{3}\left(n d_{n}\right)(k-1) / 2$. If

$$
\sup \left\{I\left(\theta, \theta_{0 n}\right) ; \theta=\theta_{n, i}+\gamma\left(\theta_{n, i}{ }^{-\theta} 0 n\right) ; \gamma \geq 0, \theta \in M\left(c_{1}\right)\right\} \geq d_{n} \text {, }
$$

define $\tilde{\theta}_{n, i}=\theta_{n, i}+\gamma_{n, i}\left(\theta_{n, i}{ }^{-\theta_{0 n}}\right)$ with $\gamma_{n, i}$ such that $I\left(\tilde{\theta}_{n, i}, \theta_{0 n}\right)=d_{n}$. If (3.4.2) does not hold, let $\tilde{\theta}_{n, i}=\theta_{0 n}$.

Consider $\theta \in M$ satisfying $I\left(\theta, \theta_{0 n}\right) \geq d_{n}$. Define $\theta^{*}$ by $\theta^{*}=\theta_{0 n}+\gamma_{n}^{*}\left(\theta-\theta_{0 n}\right), \gamma_{n}^{*} \geq 0$, and $\left\|\theta^{*}-\theta_{0 n}\right\|^{2^{n}}=c_{5}^{-1} d_{n}$ (note that $\left.I\left(\theta^{*}, \theta_{0 n}\right) \leq d_{n}\right)$ and define $\tilde{\theta}$ by $\tilde{\theta}=\theta_{0 n}+\tilde{\gamma}_{n}\left(\theta^{*}-\theta_{0 n}\right), \tilde{\gamma}_{n} \geq 1$, and $I\left(\tilde{\theta}, \theta_{0 n}\right)=d_{n}$. Since $\left\|\tilde{\theta}-\theta_{0 n}\right\|^{2} \leq c_{4}^{-1} d_{n}$ it follows that $\tilde{\gamma}_{n} \leq c_{6}$. Let $s_{n}\left(\tilde{\theta}, c_{6} n^{-\frac{1}{2}}\right)$ be the sphere with centre $\tilde{\theta}$ and radius $c_{6} n^{-\frac{n}{2}}$. There exists 
a $\theta_{n, i}\left(1 \leq i \leq p_{n}\right)$ such that $\left\|\theta_{n, i}-\theta^{*}\right\| \leq n^{-\frac{1}{2}}$. The line through $\theta_{0 n}$ and $\theta_{n, i}$ intersects $s_{n}\left(\tilde{\theta}, c_{6} n^{-\frac{1}{2}}\right)$ at a point $\bar{\theta}_{n, i}$. Then $I\left(\bar{\theta}_{n, i}, \theta_{0 n}\right)=I\left(\tilde{\theta}, \theta_{0 n}\right)+$ $+\left(\bar{\theta}_{n, i}-\tilde{\theta}\right) \cdot \Sigma_{\tilde{\theta}}\left(\tilde{\theta}-\theta_{0 n}\right)+o\left(n^{-1}\right) \geq d_{n}-c_{7} n^{-\frac{x}{2}} d_{n}^{\frac{3}{2}}$. Consider the function

$$
f(h)=I\left(\theta_{0 n}+h\left(\bar{\theta}_{n, i}-\theta_{0 n}\right), \theta_{0 n}\right) \text {. }
$$

For any $h \geq \frac{1}{2}$ such that $\theta_{\text {On }}+h\left(\bar{\theta}_{n, i}-{ }^{-\theta}{ }_{0 n}\right) \in M\left(c_{1}\right)$ it holds that

$$
\frac{d}{d h} f(h) \geq c_{8} d_{n}
$$

Hence the mean value theorem implies

$$
\begin{aligned}
& I\left(\bar{\theta}_{n, i}+c_{8}^{-1} c_{7} n^{-\frac{1}{2} d_{n}^{-\frac{1}{2}}}\left(\bar{\theta}_{n, i}-\theta_{0 n}\right), \theta_{0 n}\right)= \\
& =f\left(1+c_{8}^{-1} c_{7} n^{-\frac{1}{2}} d_{n}^{-\frac{1}{2}}\right) \\
& \geq f(1)+c_{8}^{-1} c_{7} n^{-\frac{1}{2}} d_{n}^{-\frac{1}{2}} c_{8} d_{n} \\
& =I\left(\bar{\theta}_{n, i}{ }^{, \theta}{ }_{0 n}\right)+c_{7} n^{-\frac{1}{2}} d_{n}^{\frac{1}{2}} \\
& \geq d_{n} \quad \text { for all } n \geq n_{1} \text {. }
\end{aligned}
$$

Thus (3.4.2) holds for all $\mathrm{n} \geq \mathrm{n}_{1}$. Similarly it follows that

$$
I\left(\bar{\theta}_{n, i}-c_{9} n^{-\frac{1}{2}} d_{n}^{-\frac{1}{2}}\left(\bar{\theta}_{n, i}-\theta_{0 n}\right), \theta_{0 n}\right) \leq d_{n} \quad \text { for all } n \geq n_{2} .
$$

Therefore for all $n \geq \max \left(n_{1}, n_{2}\right) \quad I\left(\tilde{\theta}_{n, i}, \theta_{0 n}\right)=d_{n}$ implies $\tilde{\theta}_{n, i}=$ $\bar{\theta}_{n, i}+n_{n}\left(\bar{\theta}_{n, i}{ }^{-\theta} 0 n\right)$, where $n_{n}$ is of order $\left(n n_{n}\right)^{-\frac{1}{2}}$, and hence $\left\|\tilde{\theta}_{n, i}-\bar{\theta}_{n, i}\right\| \leq$ $\leq c_{10}\left(n_{n}\right)^{-\frac{1}{2}}\left\|\bar{\theta}_{n, i} i^{-\theta} 0 n\right\| \leq c_{11} n^{-\frac{1}{2}}$. In combination with $\left\|\bar{\theta}_{n, i}-\tilde{\theta}\right\|=c_{6} n^{-\frac{1}{2}}$ we have $\left\|\tilde{\theta}_{n, i}-\tilde{\theta}\right\| \leq c_{2} n^{-\frac{1}{2}}$ and the proof of property (A) for all $n \geq \max \left(n_{1}, n_{2}\right)$ is complete.

Case b.

Let $L$ be a convex polytope such that $M \subset L \subset M\left(\frac{1}{2} C_{1}\right)$. Choose on the surface of $L(S L)$ points $\theta_{n, 1}, \ldots, \theta_{n, p_{n}}$ such that for all $\theta \in$ SL there exists a $\theta_{n, i}$ with $\left\|\theta-\theta_{n, i}\right\| \leq n^{-\frac{1}{2}}$, and for all $i \neq j\left\|\theta_{n, i}-\theta_{n, j}\right\|>n^{-\frac{1}{2}}$ where $i, j=1, \ldots, p_{n}$. Then $p_{n}$ is bounded above by $c_{3}\left(n d_{n}\right)(k-1) / 2$ (note that $\lim$ inf $_{n \rightarrow \infty} d_{n}>0$ ). 
(3.4.3) $\quad I\left(\theta_{n, i}, \theta_{0 n}\right)<d_{n}$

and

$$
\sup \left\{I\left(\theta, \theta_{0 n}\right) ; \theta=\theta_{n, i}+\gamma\left(\theta_{n, i}{ }^{-\theta_{0 n}}\right), \gamma \geq 0, \theta \in M\left(c_{1}\right)\right\} \geq d_{n} \text {, }
$$

then define $\tilde{\theta}_{n, i}=\theta_{n, i}+\gamma_{n, i}\left(\theta_{n, i}{ }^{-\theta_{0 n}}\right)$ with $\gamma_{n, i}$ such that $I\left(\tilde{\theta}_{n, i}, \theta_{0 n}\right)=$ $=d_{n}$. If (3.4.3) does not hold, then define $\tilde{\theta}_{n, i}=\theta_{n, i}$. Since $\theta_{0 n} \notin M\left(c_{1}\right)$ it follows that $\gamma_{n, i} \leq c_{12}$ for some $c_{12} \geq 1$.

Consider $\theta \in M$ satisfying $I\left(\theta, \theta_{0 n}\right) \geq d_{n}$. Define $\theta^{*}$ by $\theta^{*}=$ $=\theta_{0 n}+\gamma_{n}^{*}\left(\theta-\theta_{0 n}\right), 0 \leq \gamma_{n}^{*} \leq 1$, and $\theta^{*} \in$ SL. There exists a $\theta_{n, i}\left(1 \leq i \leq p_{n}\right)$ such that $\left\|\theta_{n, i^{-\frac{1}{2}}}\right\| \leq n^{-\frac{1}{2}}$. Let $s_{n}\left(\tilde{\theta}_{n, i}, c_{12^{n}}{ }^{-\frac{1}{2}}\right)$ be the sphere with centre $\tilde{\theta}_{n, i}$ and radius $c_{12} n^{-\frac{1}{2}}$. The line through $\theta_{0 n}$ and $\theta^{*}$ intersects $s_{n}\left(\tilde{\theta}_{n, i}, c_{12} n^{-\frac{1}{2}}\right)$ at a point $\bar{\theta}_{n, i}$. Then $I\left(\bar{\theta}_{n, i}, \theta_{0 n}\right)=I\left(\tilde{\theta}_{n, i}, \theta_{0 n}\right)+\left(\bar{\theta}_{n, i}-\tilde{\theta}_{n, i}\right) \cdot \Sigma_{\vartheta_{n}}\left(\vartheta_{n}-\theta_{0 n}\right)$, where $\vartheta_{n}$ lies between $\bar{\theta}_{n, i}$ and $\tilde{\theta}_{n, i}$. Consider the function

$$
f(h)=I\left(\theta_{0 n}+h\left(\bar{\theta}_{n, i}-\theta_{0 n}\right), \theta_{0 n}\right) \text {. }
$$

For any $h$ such that $\theta_{0 n}+h\left(\bar{\theta}_{n, i}{ }^{-\theta} 0 n\right) \epsilon$ int $\theta$ its derivative satisfies

$$
\frac{d}{d h} f(h)=h\left(\bar{\theta}_{n, i}{ }^{-\theta_{0 n}}\right)^{\prime} \Sigma_{\theta_{0 n}}+h\left(\bar{\theta}_{n, i}-\theta_{0 n}\right)\left(\bar{\theta}_{n, i}{ }^{-\theta_{0 n}}\right) \cdot
$$

The mean value theorem implies

$$
\begin{aligned}
& I\left(\bar{\theta}_{n, i}+c_{13} n^{-\frac{1}{2}}\left\|\bar{\theta}_{n, i}{ }^{-\theta} 0\right\|^{-1}\left(\bar{\theta}_{n, i}{ }^{-\theta} 0 n\right), \theta_{0 n}\right)= \\
& =f\left(1+c_{13} n^{-\frac{1}{2}}\left\|\bar{\theta}_{n, i}{ }^{-\theta} 0 n\right\|^{-1}\right) \\
& =I\left(\tilde{\theta}_{n, i},{ }^{\theta}{ }_{n}\right)+\left(\bar{\theta}_{n, i}-\tilde{\theta}_{n, i}\right) ' \Sigma_{\vartheta}\left(\vartheta_{n}-\theta_{0 n}\right)+ \\
& \left.+c_{13^{n}} n^{-\frac{3}{2}}\left\|\bar{\theta}_{n, i}-\theta_{0 n}\right\|^{-1}\left(1+\delta_{n}\right)\left(\bar{\theta}_{n, i}{ }^{-\theta} 0 n\right)^{\prime} \Sigma_{\theta_{0 n}}+\left(1+\delta_{n}\right)\left(\bar{\theta}_{n, i}{ }^{-\theta_{0 n}}\right)^{-\theta_{0 n}}\right) \\
& \geq I\left(\tilde{\theta}_{n, i^{\prime}} \theta_{0 n}\right) \text { for all } n \geq n_{3^{\prime}}
\end{aligned}
$$

where $0 \leq \delta_{n} \leq c_{13^{n}}{ }^{-\frac{1}{2}}\left\|\bar{\theta}_{n, i}{ }^{-\theta} 0 n\right\|^{-1}$ and the inequality is obtained by taking $c_{13}$ large enough. 
By the same line of argument

$$
\begin{aligned}
& I\left(\bar{\theta}_{n, i}-c_{14} n^{-\frac{1}{2}}\left\|\bar{\theta}_{n, i}-\theta_{0 n}\right\|^{-1}\left(\bar{\theta}_{n, i}-\theta_{0 n}\right), \theta_{0 n}\right) \leq \\
& \leq I\left(\tilde{\theta}_{n, i}, \theta_{0 n}\right) \quad \text { for all } n \geq n_{4} .
\end{aligned}
$$

Now define $\tilde{\theta}$ by $\tilde{\theta}=\bar{\theta}_{n, i}+\tilde{\gamma}_{n}\left(\bar{\theta}_{n, i}{ }^{-\theta_{0 n}}\right)$ and $I\left(\tilde{\theta}_{,} \theta_{0 n}\right)=I\left(\tilde{\theta}_{n, i},{ }_{0 n}\right)$, then $\left\|\tilde{\theta}-\bar{\theta}_{n, i}\right\| \leq \max \left(c_{13}, c_{14}\right)^{n^{-\frac{1}{2}}}$, and hence $\left\|\tilde{\theta}-\tilde{\theta}_{n, i}\right\| \leq c_{15} n^{-\frac{1}{2}}$. Let $n_{0}=$ $=\max \left(\mathrm{n}_{1}, \mathrm{n}_{2}, \mathrm{n}_{3}, \mathrm{n}_{4}\right)$, then the proof of property (A) for all $n \geq n_{0}$ is complete both in case $a$ and in case $b$.

Since it suffices to prove (3.4.1) for all $n \geq n_{0}$, assume that $\mathrm{n} \geq \mathrm{n}_{0}$. Let $\mathrm{x} \in \lambda(M)$ satisfy $I\left(\lambda^{-1}(\mathrm{x}), \theta_{0 n}\right) \geq \mathrm{d}_{\mathrm{n}}$. In view of property (A) with $\lambda^{-1}(x)$ playing the role of $\theta$ we can write $x=\lambda\left(\tilde{\theta}+\gamma\left(\tilde{\theta}-\theta_{0 n}\right)\right)$, where $\gamma \geq 0$. Consider the function $g(h)=\left(\tilde{\theta}_{n, i}-\theta_{0 n}\right) \cdot \lambda\left(\tilde{\theta}+h\left(\tilde{\theta}-\theta_{0 n}\right)\right)$, where $h \geq 0$ such that $\tilde{\theta}+h\left(\tilde{\theta}-\theta_{0 n}\right) \in M\left(c_{1}\right)$. Since its derivative is equal to

$$
\begin{aligned}
& \frac{d}{d h} g(h)=\left(\tilde{\theta}_{n, i}-\theta_{0 n}\right) \cdot \sum_{\tilde{\theta}+h\left(\tilde{\theta}-\theta_{0 n}\right)}\left(\tilde{\theta}-\theta_{0 n}\right)= \\
& =\left\|\tilde{\theta}-\theta_{0 n}\right\|^{2}\left\{\frac{\left(\tilde{\theta}_{n, i}-\tilde{\theta}\right) \cdot \sum_{\tilde{\theta}+h\left(\tilde{\theta}-\theta_{0 n}\right.}\left(\tilde{\theta}-\theta_{0 n}\right)}{\left\|\tilde{\theta}-\theta_{0 n}\right\|^{2}}+\frac{\left(\tilde{\theta}-\theta_{0 n}\right) \cdot \sum_{\tilde{\theta}+h\left(\tilde{\theta}-\theta_{0 n}\right)}\left(\tilde{\theta}-\theta_{0 n}\right)}{\left\|\tilde{\theta}-\theta_{0 n}\right\|^{2}}\right\},
\end{aligned}
$$

where the first term between the braces tends to zero as $n \rightarrow \infty$ and the second term is at least equal to $\inf \left\{u^{\prime} \Sigma_{\theta} u ;\|u\|=1, \theta \in M\left(c_{1}\right)\right\}>0, g(h)$ is an increasing function for $h \geq 0$ such that $\tilde{\theta}+h\left(\tilde{\theta}-\theta_{0 n}\right) \in M\left(c_{1}\right)$ and all sufficiently large $\mathrm{n}$. Hence

$$
\begin{aligned}
& \left(\tilde{\theta}_{n, i}{ }^{-\theta_{0 n}}\right)^{\prime} x-\psi\left(\tilde{\theta}_{n, i}\right)+\psi\left(\theta_{0 n}\right) \geq \\
& \geq\left(\tilde{\theta}_{n, i}-\theta_{0 n}\right) \cdot \lambda(\tilde{\theta})-\psi\left(\tilde{\theta}_{n, i}\right)+\psi\left(\theta_{0 n}\right) \\
& =I\left(\tilde{\theta}_{,}{ }_{0 n}\right)-I\left(\tilde{\theta}_{,} \tilde{\theta}_{n, i}\right) \geq I\left(\tilde{\theta}_{n, i}, \theta_{0 n}\right)-c_{16} n^{-1} \\
& \geq d_{n}-c_{16^{n}}{ }^{-1} .
\end{aligned}
$$

This implies that for all sufficiently large $n$ 


$$
\begin{aligned}
& P_{\theta_{O n}}\left(\bar{x}_{n} \in \lambda(M), I\left(\lambda^{-1}\left(\bar{x}_{n}\right), \theta_{0 n}\right) \geq d_{n}\right) \leq \\
& \leq \sum_{i=1}^{P_{n}} P_{\theta_{O n}}\left[\left(\tilde{\theta}_{n, i}{ }^{-\theta} O n\right) \cdot \bar{x}_{n}-\psi\left(\tilde{\theta}_{n, i}\right)+\psi\left(\theta_{0 n}\right) \geq I\left(\tilde{\theta}_{n, i}, \theta_{0 n}\right)-c_{16^{n}}{ }^{-1}\right] .
\end{aligned}
$$

From here on the last part (starting with (3.2.13)) of the proof of theorem 3.2.1 can be copied and the result is established:

LEMMA 3.4.3. Let $\mathrm{T}$ be a closed convex set in int $\Lambda$. Let $\theta \notin \lambda^{-1}(\mathrm{~T})$; define $\tilde{\theta} \in \lambda^{-1}(T)$ by $I(\tilde{\theta}, \theta)=I\left(\lambda^{-1}(T), \theta\right)$, then

$$
\text { (3.4.4) }(\tilde{\theta}-\theta)^{\prime}(x-\lambda(\tilde{\theta})) \geq 0 \text { for all } x \in T \text {. }
$$

PROOF. The set $S=\left\{x_{;} \sup _{\vartheta \in \Theta}\{\psi(\theta)-\psi(\vartheta)+(\vartheta-\theta) ' x\} \leq I(\tilde{\theta}, \theta)\right\}$ is a convex set, and so is $T$. Since $S \wedge T \neq \varnothing(\lambda(\tilde{\theta}) \in S \wedge T), S \wedge$ int $T=\varnothing$ and $\psi(\theta)-\psi(\tilde{\theta})+(\tilde{\theta}-\theta) ' x \leq I(\tilde{\theta}, \theta)$ for all $x \in S$ with equality for $x=\lambda(\tilde{\theta})$, the hyperplane $H=\left\{x ; \psi(\theta)-\psi(\tilde{\theta})+(\theta-\theta) x^{\prime}=I(\tilde{\theta}, \theta)\right\}$ is a support hyperplane of $S$. Let $H^{*}=\left\{x ; a^{\prime}(x-\lambda(\tilde{\theta}))=0\right\}$ be another support hyperplane of $s$ through $\lambda(\tilde{\theta})$. Without loss of generality assume that $\mathrm{a}^{\prime}(\lambda(\theta)-\lambda(\tilde{\theta}))>0$ (note that the case $a^{\prime}(\lambda(\theta)-\lambda(\tilde{\theta}))=0$ cannot occur since $\lambda(\theta) \in$ int $s$, because $\sup _{\vartheta \in \Theta}$ $\{(\vartheta-\theta) \cdot x-\psi(\vartheta)\}$ is a convex and hence continuous function of $x)$. For $z \in \Lambda$ Taylor expansion about $\tilde{\theta}$ yields:

and

$$
\begin{aligned}
& \left(\lambda^{-1}(z)-\theta\right)^{\prime} z-\psi\left(\lambda^{-1}(z)\right)=(\tilde{\theta}-\theta) \cdot \lambda(\tilde{\theta})-\psi(\tilde{\theta})+ \\
& +\left(\lambda^{-1}(z)-\tilde{\theta}\right) \cdot \Sigma_{\tilde{\theta}}(\tilde{\theta}-\theta)+O\left(\left\|\lambda^{-1}(z)-\tilde{\theta}\right\|^{2}\right)
\end{aligned}
$$

$$
z=\lambda\left(\lambda^{-1}(z)\right)=\lambda(\tilde{\theta})+\Sigma_{\tilde{\theta}}\left(\lambda^{-1}(z)-\tilde{\theta}\right)+O\left(\left\|\lambda^{-1}(z)-\tilde{\theta}\right\|^{2}\right) .
$$

Since $\mathrm{H} \neq \mathrm{H}^{*}$ there exists a vector $t$ with

$$
t^{\prime} \Sigma_{\tilde{\theta}}(\tilde{\theta}-\theta)<0 \quad \text { and } \quad t^{\prime} \Sigma_{\tilde{\theta}} \mathrm{a}>0
$$

Put $z=\lambda(\tilde{\theta}+\delta t)$ where $\delta$ is a positive number. If $\delta$ is small enough, then $z \in \Lambda, \psi(\theta)-\psi\left(\lambda^{-1}(z)\right)+\left(\lambda^{-1}(z)-\theta\right)^{\prime} z<I(\tilde{\theta}, \theta)$ (hence $z \in S$ ) and $a^{\prime}(z-\lambda(\tilde{\theta}))>0$. Thus we have found points of $s, \lambda(\theta)$ and $z$, in each of the two open half-spaces into which $\mathrm{H}^{*}$ separates $\mathbb{R}^{\mathrm{k}}$ : $\mathrm{H}^{\star}$ is not a support hyperplane of $\mathrm{S}$. Hence $\mathrm{H}$ separates $\mathrm{S}$ and $\mathrm{T}$, implying (3.4.4). 
PROOF OF THEOREM 3.4.1. As before we establish a relation between $\alpha_{n}$ and $d_{n}$. If the conditions of lemma 3.3.1 are not satisfied, the theorem is trivial. We therefore assume that $n d_{n} \rightarrow \infty$. In that case

(3.4.5) $\quad \alpha_{n} \leq c_{1}\left(n d_{n}\right)^{\frac{k-2}{2}} \exp \left(-n d_{n}\right)$.

(Denote by $c_{i}(i=1, \ldots, 6)$ constants with $0<c_{i}<\infty$ ) To prove (3.4.5) we first show that $x \notin \Lambda$ implies $\phi_{n}^{L R}(x)=0$. Since $d_{n}>0$ this property is obvious if $\sup _{\theta \in \theta^{\prime}}\left\{\theta^{\prime} x-\psi(\theta)\right\}=\sup _{\theta_{0} \in \theta_{0}}\left\{\theta_{0}^{\prime} x-\psi\left(\theta_{0}\right)\right\}$ for $x \notin \Lambda$. Now suppose to the contrary that $x \notin \Lambda$ and $\sup _{\theta \in \theta^{\prime}}\left\{\theta^{\prime} x-\psi(\theta)\right\}>\sup _{\theta_{0} \in \theta_{0}}\left\{\theta_{0}^{\prime} x-\psi\left(\theta_{0}\right)\right\}$, then there exists a $\tilde{\theta} \epsilon \mathrm{cl} \theta_{1}$ with $\tilde{\theta}{ }^{\prime} x-\psi(\tilde{\theta})=\sup _{\theta \in \theta^{\prime}}\left\{\theta^{\prime} x-\psi(\theta)\right\}$ and $\tilde{\theta} \epsilon$ int $\theta$. Consider the function $\theta^{\prime} x-\psi(\theta)$ in a neighbourhood of $\tilde{\theta}: \theta^{\prime} x-\psi(\theta)=$ $\tilde{\theta} \cdot x-\psi(\tilde{\theta})+(\theta-\tilde{\theta})^{\prime}(x-\lambda(\theta))-\frac{1}{2}(\theta-\tilde{\theta})^{\prime} \Sigma_{\xi}(\theta-\tilde{\theta})$, where $\xi$ lies between $\theta$ and $\tilde{\theta}$. By taking $\theta-\tilde{\theta}=\delta(x-\lambda(\tilde{\theta}))$ with $\delta>0$ sufficiently small it is easily seen that $\theta^{\prime} \mathbf{x}-\psi(\theta)>\tilde{\theta} \cdot \mathrm{x}-\psi(\tilde{\theta})$ and we have obtained a contradiction. Thus $\phi_{n}^{L R}(x)=0$ for $x \notin \Lambda$ and we can restrict our attention to points $x \in \Lambda$.

Since $K \subset$ int $\Theta$ is compact

$$
P_{\theta_{0}}\left(\bar{x}_{n} \in \lambda(K), I\left(\lambda^{-1}\left(\bar{x}_{n}\right), \theta_{0}\right) \geq d_{n}\right) \leq c_{2}\left(n d_{n}\right)^{\frac{k-2}{2}} \exp \left(-n d_{n}\right)
$$

by lemma 3.4.2. With this inequality (3.4.5) is trivial.

Now consider the MP test for this situation. Define $K(\varepsilon)$ by $K(\varepsilon)=\left\{\theta ; \inf \left\{\left\|\theta-\theta^{*}\right\| ; \theta^{*} \in \mathrm{K}\right\} \leq \varepsilon\right\}$, where $\varepsilon>0$ so small that $K(\varepsilon) \subset$ int $\theta$. Denote by $\tilde{\phi}_{n}^{+}(x)$ the critical function of the level- $\alpha_{n}$ MP test of $\tilde{\mathrm{H}}_{0}$ : $\theta \in \theta_{0} \wedge K(\varepsilon)$ against $\theta=\theta_{n} \in \theta_{1}$, then

$$
\tilde{\phi}_{n}^{+}(x)= \begin{cases}1 & \text { if } t_{n}(x)>t_{n^{\prime}} \\ 0 & \end{cases}
$$

where

$$
t_{n}(x)=\int_{\operatorname{cl~} \theta_{0} \wedge K(\varepsilon)} \exp \left\{n\left(\theta_{0}-\theta_{n}\right) ' x-n \psi\left(\theta_{0}\right)+n \psi\left(\theta_{n}\right)\right\} d \tau_{n}\left(\theta_{0}\right)
$$

and the distribution $\tau_{n}$ is least favorable. It has already be shown in the course of the proof of theorem 3.3.2 that $\left\{x ; t_{n}(x)=t_{n}\right\}$ has an empty interior. We shall prove that $\tilde{\phi}_{\mathrm{n}}^{+}$is also the MP test of the larger null hypothesis $\theta_{0}$ against $\theta=\theta_{\mathrm{n}}$.

If $E_{\theta_{n}} \tilde{\phi}_{n}^{+}\left(\bar{x}_{n}\right) \rightarrow 0$ then $E_{\theta_{n}} \phi_{n}^{+}\left(\bar{x}_{n}\right) \rightarrow 0$ and the shortcoming also tends 
to zero. Therefore assume that $E_{\theta_{n}} \tilde{\phi}_{n}^{+}\left(\bar{x}_{n}\right) \geq \delta>0$. We now show that $\left\{x ; t_{n}(x) \leq t_{n}\right\} \subset \lambda(K(\varepsilon))$ for sufficiently large $n$. For suppose that there exists a point $x_{n} \notin \lambda(K(\varepsilon))$ satisfying $t_{n}\left(x_{n}\right) \leq t_{n}$. The asymptotic normality of $\left\{\bar{x}_{n}-\lambda\left(\theta_{n}\right)\right\}_{n}^{\frac{1}{2}}$ implies that for $n$ sufficiently large

$$
P_{\theta_{n}}\left(t_{n}\left(\bar{x}_{n}\right) \leq t_{n}, \bar{x}_{n} \in B_{n}\right) \geq \frac{1}{2} \delta
$$

where $B_{n}$ is a sphere with centre $\lambda\left(\theta_{n}\right)$ and radius $c_{3} n^{-\frac{1}{2}}$. Convexity of the set $\left\{x ; t_{n}(x) \leq t_{n}\right\}$ and $x_{n} \in\left\{x ; t_{n}(x) \leq t_{n}\right\}, x_{n} \notin \lambda(K(\varepsilon))$ imply that there exists $a \theta_{0 n} \epsilon \theta_{0} \wedge K(\varepsilon)$ and a sphere $B_{n}^{*}$ with radius $c_{4}{ }^{-\frac{1}{2}}$ and centre $\lambda\left(\theta_{0 n}\right)$ contained in $\left\{x ; t_{n}(x) \leq t_{n}\right\}$. Since $\left\{x ; t_{n}(x)=t_{n}\right\}$ has an empty interior, $\tilde{\phi}_{n}^{+}(x)=1$ on int $B_{n}^{*}$ implying that $E_{\theta_{0 n}} \tilde{\phi}_{n}^{+}\left(\bar{x}_{n}\right)$ does not tend to zero, in contradiction to $\alpha_{n} \rightarrow 0$. Hence $T_{n}=\operatorname{cl}\left\{x ; \tilde{\phi}_{n}^{+}(x)>0\right\} \subset \lambda(K(\varepsilon)$ ) for all sufficiently large $\mathrm{n}$.

Let $\theta \notin K(\varepsilon)$, then by lemma 3.4 .3 there exists a point $\tilde{\theta}_{\mathrm{n}} \epsilon \lambda^{-1}\left(\mathrm{~T}_{\mathrm{n}}\right)$ such that $\left(\tilde{\theta}_{n}-\theta\right)^{\prime}\left(x-\lambda\left(\tilde{\theta}_{n}\right)\right) \geq 0$ for all $x \in T_{n}$. Let $\theta_{n}^{*}$ be the intersection of the line through $\tilde{\theta}_{\mathrm{n}}$ and $\theta$ with $K(\varepsilon): \theta_{\mathrm{n}}^{*}=\theta+\gamma_{\mathrm{n}}\left(\tilde{\theta}_{\mathrm{n}}-\theta\right)$ with $0<\gamma_{\mathrm{n}}<1$. Then $\left(\theta_{n}^{*}-\theta\right)^{\prime}\left(x-\lambda\left(\theta_{n}^{*}\right)\right)=\left(\theta_{n}^{*}-\theta\right)^{\prime}\left(x-\lambda\left(\tilde{\theta}_{n}^{j}\right)+\left(\theta_{n}^{*}-\theta\right)^{\prime}\left(\lambda\left(\tilde{\theta}_{n}\right)-\lambda\left(\theta_{n}^{*}\right)\right) \geq 0\right.$ since $\left(\theta_{n}^{*}-\theta\right)^{\prime}\left(x-\lambda\left(\tilde{\theta}_{n}\right)\right)=\gamma_{n}\left(\tilde{\theta}_{n}-\theta\right)^{\prime}\left(x-\lambda\left(\tilde{\theta}_{n}\right)\right)$ and $\left(\theta_{n}^{*}-\theta\right)^{\prime}\left(\lambda\left(\tilde{\theta}_{n}\right)-\lambda\left(\theta_{n}^{*}\right)\right)=$ $\gamma_{n}\left(1-\gamma_{n}\right)^{-1}\left(\tilde{\theta}_{n}-\theta_{n}^{*}\right)^{\prime}\left(\lambda\left(\tilde{\theta}_{n}\right)-\lambda\left(\theta_{n}^{*}\right)\right) \geq 0$. Therefore $\psi(\theta)-\psi\left(\theta_{n}^{*}\right)+\left(\theta_{n}^{*}-\theta\right)^{\prime} x \geq$ $I\left(\theta_{n}^{*}, \theta\right)>0$, and hence

$$
\theta^{\prime} x-\psi(\theta) \leq \theta_{n}^{*} x-\psi\left(\theta_{n}^{*}\right) \text { for all } x \in T_{n} \cdot
$$

It follows that

$$
\begin{aligned}
E_{\theta} \tilde{\phi}_{n}^{+}\left(\bar{x}_{n}\right) & =\int \tilde{\phi}_{n}^{+}(x) \exp \left\{n\left(\theta-\theta_{n}^{*}\right)^{\prime} x-n \psi(\theta)+n \psi\left(\theta_{n}^{*}\right)\right\} d \bar{P}_{\theta_{n}^{*}}^{-n}(x) \\
& \leq E_{\theta_{n}^{*}} \tilde{\phi}_{n}^{+}\left(\bar{x}_{n}\right) \leq \alpha_{n}
\end{aligned}
$$

for all $n \geq n_{0}$ where $n_{0}$ does not depend on $\theta$. This implies that $\tilde{\phi}_{n}^{+}$is also the critical function of the MP size- $\alpha_{n}$ test of $\mathrm{H}_{0}: \theta \in \theta_{0}$ against $\theta=\theta_{\mathrm{n}} \in \Theta_{1}$. So we have essentially reduced the MP test of $\mathrm{H}_{0}: \theta \epsilon \theta_{0}$ to a MP test of a null hypothesis contained in a compact subset of int $\theta$. Following the same line of argument we used in part a of the proof of theorem 3.3.2 the assumption $\mathrm{R}_{n}\left(\theta_{n}\right) \geq \eta>0$ again leads to the inequality $\alpha_{n} \geq c_{5} \exp \left(-n d_{n}+c_{6} n^{\frac{1}{2}} d_{n}^{\frac{1}{2}}\right)$, in contradiction to (3.4.5) and the proof of the theorem is complete. 
3.5. UNIFORM CONVERGENCE ON COMPACT SUBSETS OF INT $\theta$

In the one-dimensional case of chapter II we showed that $R_{n}(\theta)$ tends to zero uniformly on the intersection of $\theta_{1}$ with a compact subset of int $\theta$ without any condition at all. Unfortunately we do not know whether this result holds in the $k$-dimensional case and we therefore prove a generalization of theorem 2.7.1 under some assumptions. It turns out that in some (classical) testing problems this theorem can be applied. In this section we assume that $\dot{\theta}_{0}$ is a Borel set. Consider the testing problem $\mathrm{H}_{0}: \theta \in \theta_{0}$ against $\mathrm{H}_{1}$ : $\theta \notin \theta_{0}$.

ASSUMPTION A1. FOr all $\mathrm{n}$ the LR test satisfies

$$
\frac{\sup _{\theta_{0} \epsilon \Theta_{0} \wedge K} E_{\theta_{0}} \phi_{n}^{L R}\left(\bar{x}_{n}\right)}{\sup _{\theta \in \Theta_{0}} E_{\theta} \phi_{n}^{L R}\left(\bar{x}_{n}\right)} \geq \varepsilon_{1}
$$

for some compact subset $\mathrm{K}$ of int $\theta$ and some $\varepsilon_{1}>0$.

\section{ASSUMPTION A2.}

$$
\alpha_{n} \geq \exp (-n I)
$$

for all sufficiently large $\mathrm{n}$ and some $\mathrm{I}<\mathrm{I}\left(\theta_{0} \wedge \mathrm{K}\right)(\mathrm{cf} \cdot(3.2 .1))$.

Assumption A1 states that the size $\alpha_{n}$ (or a fixed part of it) is reached at parameter points bounded away from the boundary of the parameter space.

It is clear (cf. lemma 3.3.3) that when these two assumptions are fulfilled

(3.5.1) $\alpha_{n} \leq c\left(n d_{n}\right)^{l} \exp \left(-n d_{n}\right)$, where $\ell=(k-2) / 2$ and $c$ some positive constant.

We now prove the main theorem of this section.

THEOREM 3.5.1. Let $\mathrm{L}$ be an arbitrary compact subset of int $\theta$. If (3.5.1) holds for some fixed \& then $\lim _{n \rightarrow \infty} R_{n}(\theta)=0$ uniformly on $L \wedge \theta_{1}$.

PROOF. We may assume that $\lambda(0)=0$ and thus $\psi(\theta) \geq 0$ for all $\theta \in \theta$. Let I be 
a compact subset of int $\theta$. Let $\left\{\theta_{n}\right\}$ be a sequence of points in $L, \theta_{n} \notin \operatorname{cl} \theta_{0}$ and $R_{n}\left(\theta_{n}\right) \geq \varepsilon_{2}>0$. For sufficiently large $n$ there exists a sphere $B_{n}$ with centre $\lambda\left(\theta_{n}\right)$ and radius $c_{1} n^{-\frac{1}{2}}$ such that $P_{\theta_{n}}\left(\bar{x}_{n} \in B_{n}\right) \geq 1-\frac{1}{2} \varepsilon_{2}$ and hence

$$
\int_{B_{n} \wedge I\left(\lambda^{-1}(x), \theta_{0}\right) \leq d_{n}} \phi_{n}^{+}(x) d \bar{P}_{\theta_{n}^{-n}}(x) \geq \frac{1}{2} \varepsilon_{2} .
$$

Here again denote by $c_{i}(i=1, \ldots, 5)$ constants with $0<c_{i}<\infty$. The critical function of the MP test is of the form

$$
\phi_{n}^{+}(x)=\left\{\begin{array}{ll}
1 & \lim \inf \int t_{n}\left(\theta_{0}, x\right) d \lambda_{i, n}\left(\theta_{0}\right)< \\
\text { if } & \lim _{i \rightarrow \infty} \sup \int t_{n}\left(\theta_{0}, x\right) d \lambda_{i, n}\left(\theta_{0}\right)>
\end{array},\right.
$$

where $t_{n}\left(\theta_{0}, x\right)=\exp \left\{n\left(\theta_{0}-\theta_{n}\right) \cdot x+n \psi\left(\theta_{n}\right)\right\}$ and $\lambda_{i, n}$ is a measure satisfying $\lambda_{i, n}\left(\mathbb{R}^{\mathrm{k}}\right)=\lambda_{i, \mathrm{n}}\left(\theta_{0}\right)$ and $\int \exp \left\{\mathrm{n} \psi\left(\theta_{0}\right)\right\} \mathrm{d} \lambda_{i, n}\left(\theta_{0}\right) \leq \alpha_{\mathrm{n}}^{-1}(i \in \mathbb{N})$ (see [14]). Let

and

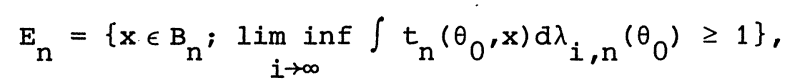

$$
F_{n}=\left\{x \in B_{n} ; \lim _{i \rightarrow \infty} \sup _{j} \int t_{n}\left(\theta_{0}, x\right) d \lambda_{i, n}\left(\theta_{0}\right) \leq 1\right\}
$$

These sets have the following properties:

(i) $\mathrm{F}_{\mathrm{n}}$ is a convex set,

(ii) $\lim _{\mathrm{n} \rightarrow \infty} P_{\theta_{\mathrm{n}}}\left(\bar{x}_{\mathrm{n}} \in \mathrm{E}_{\mathrm{n}} \wedge \mathrm{F}_{\mathrm{n}}\right)=0$.

This second property means that the set on which randomization is possible tends to zero in $P_{\theta_{n}}$-probability. The first property is an immediate consequence of the convexity of the integrand.

The proof of (ii) is similar to that part of the proof of theorem 3.3.2, where it is shown that int $\left(U_{2, n}-U_{1, n}\right)=\varnothing$. But here the situation is more complicated since we have to deal with a sequence of measures $\left\{\lambda_{i, n}\right\}$.

If $x \in E_{n} \wedge F_{n}$ we have $\lim _{i \rightarrow \infty} \int t_{n}\left(\theta_{0}, x\right) d \lambda_{i, n}\left(\theta_{0}\right)=1$ and hence by Fatou's lemma and Fubini's theorem

$$
\text { (3.5.3) } P_{\theta_{n}}\left(\bar{x}_{n} \in E_{n}^{\wedge F_{n}}\right)=\int_{E_{n} \wedge F_{n}} \lim _{i \rightarrow \infty} \int_{\theta_{0}} t_{n}\left(\theta_{0}, x\right) d \lambda_{i, n}\left(\theta_{0}\right) d \bar{P}_{\theta_{n}}^{n}(x) \leq
$$




$$
\begin{aligned}
& \leq \underset{i \rightarrow \infty}{\lim \inf } \int_{E_{n} \wedge F_{n}} \int_{\theta_{0}} t_{n}\left(\theta_{0}, x\right) d \lambda_{i, n}\left(\theta_{0}\right) d \bar{P}_{\theta_{n}}^{-n}(x) \\
& =\underset{i \rightarrow \infty}{\lim \inf } \int_{\theta_{0}} \int_{E_{n} \wedge F_{n}} t_{n}\left(\theta_{0}, x\right) d \bar{P}_{\theta_{n}}^{n}(x) d \lambda_{i, n}\left(\theta_{0}\right) \\
& =\underset{i \rightarrow \infty}{\lim \inf } \int_{\theta_{0}} P_{\theta_{0}}\left(\bar{x}_{n} \in E_{n} \wedge F_{n}\right) \exp \left\{n \psi\left(\theta_{0}\right)\right\} d \lambda_{i, n}\left(\theta_{0}\right) .
\end{aligned}
$$

The sequence $\left\{\lambda_{i, n} ; i \in \mathbb{N}\right\}$ is a sequence of uniformly bounded measures: $\lambda_{i, n}\left(\mathbb{R}^{k}\right) \leq \alpha_{n}^{-1}$. Hence there exists a subsequence $\left\{\lambda_{i_{j}, n}\right\}$ and a measure $\nu_{n}$ such that $\lambda_{i_{j}, n} \rightarrow \nu_{n}$ vaguely. Assume that the Lebesgue measure of $\mathrm{E}_{\mathrm{n}} \wedge \mathrm{F}_{\mathrm{n}}$ is positive (otherwise $\lim _{\mathrm{n} \rightarrow \infty} \mathrm{P}_{\theta_{\mathrm{n}}}^{-\mathrm{n}}\left(\mathrm{E}_{\mathrm{n}} \wedge \mathrm{F}_{\mathrm{n}}\right)=0$ by asymptotic normality). Then there exist, for any fixed $n$, points $x, y_{1}, \ldots, y_{k}$ in $E_{n} \wedge F_{n}$ with the following two properties: $x-y_{1}, \ldots, x-y_{k}$ are linear independent and there are $\alpha_{1}, \ldots, \alpha_{k} \neq 0$ or 1 with $\alpha_{\mathrm{s}} \mathrm{x}+\left(1-\alpha_{\mathrm{s}}\right) \mathrm{y}_{\mathrm{s}} \in \mathrm{E}_{\mathrm{n}} \wedge \mathrm{F}_{\mathrm{n}}(\mathrm{s}=1, \ldots, \mathrm{k})$.

Let $T_{s}=\left\{\theta_{0} ;\left(\theta_{0}-\theta_{n}\right)^{\prime} x=\left(\theta_{0}-\theta_{n}\right)^{\prime} y_{s}\right\} \quad(s=1, \ldots, k)$. By definition of $E_{n} \wedge F_{n}$ and convexity of $t_{n}\left(\theta_{0}, \cdot\right)$ it follows that

$$
\begin{aligned}
0= & \lim _{j \rightarrow \infty} \int \alpha_{s} t_{n}\left(\theta_{0}, x\right)+\left(1-\alpha_{s}\right) t_{n}\left(\theta_{0}, y_{s}\right)- \\
& \quad-t_{n}\left(\theta_{0}, \alpha_{s} x+\left(1-\alpha_{s}\right) y_{s}\right) d \lambda_{i_{j}, n}\left(\theta_{0}\right)
\end{aligned}
$$

and therefore

$$
\int_{T_{s}^{c}}\left|\alpha_{s} t_{n}\left(\theta_{0}, x\right)+\left(1-\alpha_{s}\right) t_{n}\left(\theta_{0}, y_{s}\right)-t_{n}\left(\theta_{0}, \alpha_{s} x+\left(1-\alpha_{s}\right) y_{s}\right)\right| d \nu_{n}\left(\theta_{0}\right)=0 .
$$

on $T_{S}^{C}$ the integrand is positive $\left(\alpha_{s} \neq 0\right.$ or 1$)$, hence $\nu_{n}\left(T_{s}^{C}\right)=0(s=1, \ldots, k)$ and thus $\nu_{n}\left(U_{s=1}^{k} T_{s}^{C}\right)=0$. Since $x-y_{1}, \ldots, x-y_{k}$ are linear independent, $U_{S=1}^{k} T_{S}^{C}=\mathbb{R}^{k}-\left\{\theta_{n}\right\}$. Note that $\theta_{n} \notin c l \theta_{0}$ and hence for each compact set $G$

$$
\text { (3.5.4) } \lim _{j \rightarrow \infty} \lambda_{i_{j}, n}\left(G \wedge \mathrm{cl} \theta_{0}\right)=\nu_{n}\left(G \wedge \mathrm{cl} \theta_{0}\right)=0 \text {. }
$$


For all $n \geq n_{0} \lim _{j \rightarrow \infty} \psi\left(\vartheta_{j}\right)=\infty$ implies $\lim _{j \rightarrow \infty} I\left(\lambda^{-1}\left(B_{n}\right), \vartheta_{j}\right)=\infty$ (cf. lemma 4.1.2). From now on let $n \geq n_{0}$. By lemma 3.4.2 for all $A>0$ it holds that

$$
P_{\theta_{0}}\left(\overline{\mathrm{X}}_{\mathrm{n}} \in \mathrm{E}_{\mathrm{n}} \wedge \mathrm{F}_{\mathrm{n}}\right) \leq P_{\theta_{0}}\left(\overline{\mathrm{X}}_{\mathrm{n}} \in \mathrm{B}_{\mathrm{n}}\right) \leq \mathrm{c}_{2}(\mathrm{nA})^{(\mathrm{k}-2) / 2} \exp (-\mathrm{nA})
$$

for all $\theta_{0}$ satisfying $I\left(\lambda^{-1}\left(B_{n}\right), \theta_{0}\right)>A$. In combination with $(3.5 .3)$ and (3.5.4) we therefore obtain $P_{\theta_{n}}\left(\bar{X}_{n} \in E_{n} \wedge F_{n}\right)=0$ if the Lebesgue measure of $E_{n} \wedge F_{n}$ is positive. This completes the proof of (ii).

As in the proof of theorem 3.3.2 we select a point $\zeta_{n}$ such that

(a) $\lambda\left(\zeta_{n}\right) \in B_{n^{\prime}}$

(b) $I\left(\zeta_{n}, \theta_{0}\right) \leq d_{n}$,

(c) there exists a sphere $B_{n}^{*}$ with centre $\lambda\left(\zeta_{n}\right)$ and radius $c_{3} n^{-\frac{1}{2}}$ contained in $\mathrm{F}_{\mathrm{n}} \wedge \mathrm{B}_{\mathrm{n}}$.

Following the same line of proof as in theorem 3.3.2 we find once more $\alpha_{n} \geq c_{4} \exp \left\{-n d_{n}+c_{5}\left(n d_{n}\right)^{\frac{1}{2}}\right\}$, in contradiction to (3.5.1), which completes the proof of the theorem.

As an immediate consequence we have

THEOREM 3.5.2. Let $\mathrm{L}$ be an arbitrary compact subset of int $\Theta$. If the assumptions $\mathrm{A} 1$ and $\mathrm{A} 2$ are fulfilled, then the shortcoming of the LR test tends to zero uniformly on $\mathrm{L} \wedge \theta_{1}$.

As applications of theorem 3.5.2 we consider two (classical) testing problems concerning the normal distribution. Let $\left\{x_{n}\right\}$ be a sequence of normally distributed random variables with mean $\mu$ and variance $\sigma^{2}$. We first consider the testing problem $\mathrm{H}_{0}: \mu=\mu_{0}$ against $\mathrm{H}_{1}: \mu \neq \mu_{0}$, where $\mu_{0}$ is some constant $\left(-\infty<\mu_{0}<\infty\right)$ and $\sigma^{2}$ is unspecified. The LR test (i.e. the two-sided t-test) is similar; hence assumption A1 is fulfilled for every compact $K$, furthermore $I\left(\theta_{0} \wedge K\right)=\infty$. Therefore if $-n^{-1} \log \alpha_{n}$ is bounded above, the shortcoming of the LR test tends to zero uniformly on the intersection of $\theta_{1}$ with a compact subset of (int) $\theta\left(\theta\right.$ corresponds to $\left.-\infty<\mu<\infty, 0<\sigma^{2}<\infty\right)$.

REMARK 3.5.1. If $-\mathrm{n}^{-1} \log \alpha_{\mathrm{n}}$ is unbounded, the envelope power function tends to zero uniformly on every compact subset of $\theta$. More general: if $\sup _{\theta \in \mathrm{L}} I\left(\theta, \Theta_{0}\right)<I\left(\Theta_{0} \wedge \mathrm{K}\right)$ for all compact subsets $L \subset$ int $\theta$, then assumption $\mathrm{A} 2$ is redundant in theorem 3.5.2.

Hence we obtain 
COROLLARY 3.5.3. Let $\mathrm{L}$ be an arbitrary compact subset of $\theta$. The shortcoming of the t-test tends to zero uniformly on $L \wedge \theta_{1}$.

The second testing problem concerns the variance $\sigma^{2}, \mathrm{H}_{0}: \sigma^{2}=\sigma_{0}^{2}$ against $\mathrm{H}_{1}$ : $\sigma^{2} \neq \sigma_{0}^{2}$, where $\sigma_{0}^{2}$ is some constant $\left(0<\sigma_{0}^{2}<\infty\right)$. The LR test rejects $\mathrm{H}_{0}$ if

$$
\sigma_{0}^{-2} s_{n}^{2}-\log \left(\sigma_{0}^{-2} s_{n}^{2}\right)>2 d_{n}+1
$$

where $s_{n}^{2}=n^{-1} \sum_{i=1}^{n}\left(x_{i}-\bar{x}_{n}\right)^{2}$. Again the LR test is similar and assumption $A 1$ is fulfilled for every compact $K$, moreover $I\left(\theta_{0} \wedge K\right)=\infty$. Remark 3.5 .1 also applies in this case. Hence

COROLLARY 3.5.4. Let $\mathrm{L}$ be an arbitrary compact subset of $\Theta$. The shortcoming of the LR test for the testing problem $\mathrm{H}_{0}: \sigma^{2}=\sigma_{0}^{2}$ against $\mathrm{H}_{1}: \sigma^{2} \neq \sigma_{0}^{2}$ tends to zero uniformly on $L \wedge \theta_{1}$.

3.6. THE k-DIMENSIONAL NORMAL DISTRIBUTION WITH KNOWN COVARIANCE MATRIX

For multivariate normal distributions with known covariance matrix we have the following strong result:

THEOREM 3.6.1. Let $\mathrm{x}_{1}, \mathrm{x}_{2}, \ldots$ be i.i.d. random $\mathrm{k}$-dimensional vectors normally distributed with known covariance matrix. Consider the testing problem $\mathrm{H}_{0}: \mu \in \mathrm{M}_{0}$ against $\mathrm{H}_{1}: \mu \notin \mathrm{M}_{0}$ where $\mu=\mathrm{EX}_{1}$ and $\mathrm{M}_{0}$ is an arbitrary subset of $\mathbb{R}^{k}$. Then the shortcoming of the LR test tends to zero uniformly on $\mathbb{R}^{\mathrm{k}}-\mathrm{M}_{0}$.

PROOF. Since we investigate an arbitrary null hypothesis, we assume without loss of generality that the covariance matrix is the identity: $I_{k}$.

Then the dominating measure appearing in the definition of exponential families corresponds to the multivariate normal $N\left(0, I_{k}\right)$ distribution and $\theta$ corresponds to $\mu$. The functions $\psi, \lambda$ and $I$ are given by

$$
\psi(\theta)=\frac{1}{2}\|\theta\|^{2}, \quad \lambda(\theta)=\theta \text { and } I(\theta, \tilde{\theta})=\frac{1}{2}\|\theta-\tilde{\theta}\|^{2} .
$$

Hence the LR test has the following form:

$$
\phi_{n}^{L R}(x)=\left\{\begin{array}{lll}
1 & \text { if inf }{ }_{\theta_{0} \in \Theta_{0}}\left\|x-\theta_{0}\right\|^{2} & 2 d_{n} .
\end{array}\right.
$$


If the conditions of lemma 3.3.1 are not fulfilled the theorem is trivial; so assume that $n d_{n} \rightarrow \infty$. We investigate the relation between $\alpha_{n}$ and $d_{n}$.

$$
\begin{aligned}
& \text { (3.6.1) } \quad \alpha_{n}=\sup _{\theta_{0} \in \Theta_{0}} P_{\theta_{0}}\left(\text { inf }_{\vartheta \in \Theta_{0}}\left\|\bar{x}_{n}-\vartheta\right\|^{2} \geq 2 d_{n}\right) \\
& \leq \sup _{\theta_{0} \in \Theta_{0}} P_{\theta_{0}}\left(\left\|\bar{x}_{n}-\theta_{0}\right\|^{2} \geq 2 d_{n}\right) \\
& =\int_{2 n d}^{\infty} \frac{e^{-\frac{1}{2} x} \cdot x^{\frac{k-2}{2}}}{\Gamma\left(\frac{1}{2} k\right) 2^{\frac{1}{2} k}} d x \\
& \leq c_{1}\left(n d_{n}\right)^{\frac{k-2}{2}} \exp \left(-n d_{n}\right) \text {. }
\end{aligned}
$$

Denote by $c_{1}, c_{2}$ and $c_{3}$ positive constants. For the remainder of the proof we follow the same line of argument as in theorem 3.3.2: again there exists a least favorable distribution (see LEHMANN (1959) section 3.8), and using the concrete form of $I(\theta, \tilde{\theta})$ the existence of the points $\zeta_{n}, \xi_{n}$ and $n_{n}$ is guaranteed even if $\left\|\theta_{n}\right\| \rightarrow \infty$. Hence if $R_{n}\left(\theta_{n}\right) \geq \varepsilon$ for some sequence $\left\{\theta_{n}\right\}$ we find

$$
\alpha_{n} \geq c_{2} \exp \left\{-n d_{n}+c_{3} n^{\frac{1}{2}} d_{n}^{\frac{1}{2}}\right\}
$$

in contradiction to $(3.6 .1)$, which completes the proof.

\subsection{THE MULTINOMIAL DISTRIBUTION}

At the beginning of the work on large deviations and shortcomings of LR tests were the papers of HOEFFDING (1965a) and OOSTERHOFF and VAN ZWET (1970) devoted to the multinomial distribution. In this section we extend the results of the latter paper to quite general null hypotheses.

We start with some notations. The random $k$-dimensional vector $Y_{n}$ is said to have a $k$-dimensional multinomial distribution with parameters $\mathrm{n}$ and $p=\left(p^{(1)}, \ldots, p^{(k)}\right)$ if

(3.7.1) $\quad P_{p}\left(Y_{n}=y\right)=\frac{n !}{y^{(1)} ! \ldots y^{(k)} !} j_{j=1}^{k} p^{(j)^{y^{(j)}}}$,

where $y=\left(y^{(1)}, \ldots, y^{(k)}\right)$ has non-negative integer components with 
sum $\mathrm{n}$ and $\mathrm{p}$ is any point in the simplex

$$
\Pi=\left\{\left(z^{(1)}, \ldots, z^{(k)}\right) ; \sum_{j=1}^{k} z^{(j)}=1, z^{(j)} \geq 0 \text { for } j=1, \ldots, k\right\} .
$$

As we have already seen in example 2.5.1 the shortcoming of the LR test does not necessarily tend to zero uniformly over the whole set of alternatives. However, we can prove the following result:

THEOREM 3.7.1. Let $\mathrm{Y}_{\mathrm{n}}$ be a random vector having a k-dimensional multinomial distribution with parameters $\mathrm{n}$ and $\mathrm{p}=\left(\mathrm{p}^{(1)}, \ldots, \mathrm{p}^{(\mathrm{k})}\right), \mathrm{n}=1,2, \ldots$. Consider the testing problem $\mathrm{H}_{0}: \mathrm{p} \in \Pi_{0}$ against $\mathrm{H}_{1}: \mathrm{p} \in \Pi_{1}=\Pi-\Pi_{0}$, where $\Pi_{0}$ is a subset of $\Pi$ with the property $p \in\left\{p \in \Pi_{i} ; p^{(j)}=0\right.$ for some $\left.j\right\}$ implying $p \in c l$ (int $\Pi_{i}$ ), $i=0,1$. Let $L$ be an arbitrary compact subset of

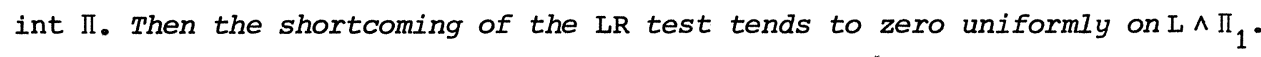
(Note that the condition on $\pi_{0}$ implies that no boundary point of $\pi$ is an isolated point of either $\Pi_{0}$ or $\Pi_{1}$.)

PROOF. In view of the property of $\pi_{0}$ the LR test statistic does not change if the parameter space $\Pi$ is restricted to int $\Pi$. Moreover since $P_{p}\left(Y_{n} \in A\right)$ is a continuous function of $p$ for every region $A$ and all $n \in \mathbb{N}$ the LR test of

$$
H_{0}^{\prime}: p \in \Pi_{0}^{\prime} \stackrel{\text { def }}{=} \Pi_{0} \wedge \text { int } \Pi
$$

against

$$
H_{1}^{\prime}: p \in \Pi_{1}^{\prime} \stackrel{\text { def }}{=}(\text { int } \Pi)-\Pi_{0}
$$

is fully equivalent to the LR test of the original problem. Furthermore the envelope power functions of both testing problems are identical because a MP test of $\mathrm{H}_{0}^{\prime}$ against a simple alternative is also a MP test of $\mathrm{H}_{0}$ against this alternative. In the sequel we therefore consider the problem of testing $\mathrm{H}_{0}^{\prime}$ against $\mathrm{H}_{1}^{\prime}$.

In this situation the multinomial distribution can be brought in the form of a $(k-1)$-parameter exponential family by the introduction of new parameters

$$
\theta^{(j)}=\log \left(p^{(j)} / p^{(k)}\right) \quad j=1, \ldots, k-1 .
$$

The LR test of $H_{0}^{\prime}$ against $H_{1}^{\prime}$ is of the following form: 


$$
\phi_{n}^{L R}(y)=\left\{\begin{array}{lll}
1 & > \\
0 & \text { if inf } \\
0 & <\Pi_{0}^{\prime} I_{k}(y, p) & d_{n},
\end{array}\right.
$$

where $I_{k}(y, p)=\sum_{i=1}^{k} \bar{y}^{(i)} \log \left(\bar{y}^{(i)} / p(i)\right.$ ) with the convention that $r \log (r / s)=0$ if $r=0$ and where $\bar{y}^{(i)}$ is defined by $\bar{y}^{(i)}=n^{-1} y^{(i)}$.

If the conditions of lemma 3.3.1 are not satisfied, the theorem is trivial. We therefore assume that $n d_{n} \rightarrow \infty$.

We shall prove that $\sup _{p \in \Pi_{0}^{\prime}} E_{p} \phi_{n}^{L R}\left(Y_{n}\right) \leq c\left(n d_{n}\right)^{(k-2) / 2} \exp \left(-n d_{n}\right)$. For this purpose it is sufficient to show that if $\operatorname{nd}_{n} \geq \varepsilon>0$

(3.7.2) $\quad P_{p}\left(I_{k}\left(Y_{n}, p\right) \geq d_{n}\right) \leq c_{k}\left(n d_{n}\right)^{\frac{k-2}{2}} \exp \left(-n d_{n}\right)$

for $p \in$ int $\Pi$ where $c_{k}$ is a positive constant independent of $p$ and $n$. The proof is by induction on $\mathrm{k}$.

For $\mathrm{k}=2$ the multinomial distribution reduces to a binomial distribution and lemma 2.3.1 yields

$$
P_{p}\left(I_{2}\left(Y_{n}, p\right) \geq d_{n}\right) \leq 5 \exp \left(-n d_{n}\right)
$$

Suppose that (3.7.2) is true for $\mathrm{k}$ and let $\mathrm{Y}_{\mathrm{n}}=\left(\mathrm{Y}_{\mathrm{n}}^{(1)}, \ldots, \mathrm{Y}_{\mathrm{n}}^{(\mathrm{k}+1)}\right)$ have a $(k+1)$-dimensional multinomial distribution. Then

$$
\begin{aligned}
& P_{p}\left(\sum_{i=1}^{k+1} \bar{Y}_{n}^{(i)} \log \left(\bar{Y}_{n}^{(i)} / p(i)\right) \geq d_{n}\right)= \\
& =P_{p}\left(Y_{n}^{(1)}=n,-\log p^{(1)} \geq d_{n}\right)+\sum_{j=0}^{n-1} P_{p}\left(Y_{n}^{(1)}=j\right) \times \\
& \times P P_{p}\left[\sum_{i=2}^{k+1} \frac{n}{n-j} \bar{Y}_{n}^{(i)} \log \left\{\frac{n}{n-j} \bar{Y}_{n}^{(i)} / \tilde{p}^{(i)}\right\} \geq \frac{n}{n-j}\left(d_{n}-I^{*}\left(n^{-1} j, p_{1}\right)\right\} \mid Y_{n}^{(1)}=j\right],
\end{aligned}
$$

where $\overline{\mathrm{Y}}_{\mathrm{n}}^{(\mathrm{i})}=\mathrm{n}^{-1} \mathrm{Y}_{\mathrm{n}}^{(i)}(i=1, \ldots, k+1), I^{*}(r, s)=r \log (r / s)+$

$+(1-r) \log \{(1-r) /(1-s)\}=I_{2}((n r, n-n r),(s, 1-s))$ and $\tilde{p}^{(i)}=p^{(i)} /(1-p(1))$ $(i=2, \ldots, k+1)$. The first term in the sum in (3.7.3) is bounded above by $\exp \left(-n d_{n}\right)$ and we therefore restrict our attention to the second term. We split this sum in two parts by the introduction of the following sets of indices:

and

$$
J_{1, n}=\left\{0 \leq j \leq n-1 ; I^{*}\left(n^{-1} j, p(1)\right) \leq d_{n}-\frac{1}{2} n^{-1}(k-1) \log \left(n d_{n}\right)\right\}
$$




$$
J_{2, n}=\left\{0 \leq j \leq n-1 ; I^{*}\left(n^{-1} j, p^{(1)}\right)>d_{n}-\frac{1}{2} n^{-1}(k-1) \log \left(n d_{n}\right)\right\} .
$$

By lemma 2.3.1 we have the following inequality

$$
\begin{aligned}
& \sum_{j \in J_{2, n}} P_{p}\left(Y_{n}^{(1)}=j\right) \leq \\
& \leq P_{p}\left[I^{*}\left(\bar{Y}_{n}^{(1)}, p(1)\right)>d_{n}-\frac{1}{2} n^{-1}(k-1) \log \left(n d_{n}\right)\right] \\
& \leq 5\left(n d_{n}\right)^{\frac{k-1}{2}} \exp \left(-n d_{n}\right) .
\end{aligned}
$$$$
\text { Let } c_{k+1} \geq \min \left\{e^{t} t^{-((k-1) / 2)} ; \varepsilon \leq t \leq e^{2 \varepsilon}\right\} ; \text { then obviously (3.7.2) }
$$

holds if $n d_{n} \in\left[\varepsilon, e^{2 \varepsilon}\right]$. We therefore assume that $n d_{n} \geq e^{2 \varepsilon}$. This implies that

$$
\begin{aligned}
& (n-j) \frac{n}{n-j}\left\{d_{n}-I^{\star}\left(n^{-1} j, p(1)\right)\right\} \geq \frac{1}{2}(k-1) \log n d_{n} \geq \\
& \geq \frac{1}{2}(k-1) 2 \varepsilon \geq \varepsilon
\end{aligned}
$$

for $j \in J_{1, n}$. Now the induction hypothesis can be applied for $j \in J_{1, n^{\prime}}$ because conditional on $\mathrm{Y}_{\mathrm{n}}^{(1)}=\mathrm{j}$ the vector $\left(\mathrm{Y}_{\mathrm{n}}^{(2)}, \ldots, \mathrm{Y}_{\mathrm{n}}^{(\mathrm{k}+1)}\right.$ ) has a $\mathrm{k}$-dimensional multinomial distribution with parameters $n-j$ and $\tilde{p}=\left(\tilde{p}^{(2)}, \ldots, \tilde{p}^{(k+1)}\right)$ and (3.7.4) holds. Hence

$$
\begin{aligned}
& P_{p}\left(\sum_{i=1}^{k+1} \bar{Y}_{n}^{(i)} \log \left(\bar{Y}_{n}^{(i)} / p(i)\right) \geq d_{n}\right) \leq \\
& \leq\left\{1+5\left(n d_{n}\right)^{\frac{k-1}{2}}\right\} \exp \left(-n d_{n}\right)+ \\
& +\sum_{j \in J_{1, n}} P_{p}\left(Y_{n}^{(1)}=j\right) c_{k}\left(n d_{n}\right)^{\frac{k-2}{2}} \exp \left\{-n d_{n}+n I^{*}\left(n^{-1} j, p(1)\right)\right\} .
\end{aligned}
$$

So we proceed to analyse this last sum. Since $P_{p}\left(Y_{n}^{(1)}=j\right)=\operatorname{Pr}\left(X_{j n}=j\right) \times$ $\exp \left\{-n I^{*}\left(n^{-1} j, p^{(1)}\right)\right\}$, where $x_{j n}$ has a binomial distribution with parameters $\mathrm{n}$ and $\mathrm{n}^{-1} j$, it remains to prove that

$$
\text { (3.7.5) } \sum_{j \in J_{1, n}} \operatorname{Pr}\left(x_{j n}=j\right) \leq \tilde{c}\left(\text { nd }_{n}\right)^{\frac{1 / 5}{2}}
$$

for some positive constant $\tilde{c}$. For reason of symmetry it is no restriction to 
assume that $\mathrm{p}^{(1)} \leq \frac{1}{2}$. The inequality

$$
(2 \pi)^{\frac{1}{2} n} n^{n+\frac{1}{2}} e^{-n}<n !<(2 \pi)^{\frac{1}{2}} n^{n+\frac{1}{2}} e^{-n}\left(1+(4 n)^{-1}\right)
$$

yields the upper bound

$$
\operatorname{Pr}\left(x_{j n}=j\right) \leq\left\{\frac{n}{j(n-j)}\right\}^{\frac{1}{2}} \quad(1 \leq j \leq n-1) .
$$

Since

$$
\begin{aligned}
& \sum_{j=1}^{n-1}\left\{\frac{n}{j(n-j)}\right\}^{\frac{1}{2}} \leq 2 \sum_{1 \leq j \leq \frac{1}{2} n}\left\{\frac{n}{j(n-j)}\right\}^{\frac{1}{2}} \leq 2^{3 / 2} \sum_{1 \leq j \leq \frac{1}{2} n} j^{-\frac{1}{2}} \leq \\
& \leq 2^{3 / 2} \int_{0}^{\frac{1}{2} n} z^{-\frac{1}{2}} d z=4 n^{\frac{1}{2}},
\end{aligned}
$$

it follows that

$$
\sum_{j=0}^{n-1} \operatorname{Pr}\left(x_{j n}=j\right) \leq 4 n^{\frac{1}{2}}+1 \leq 5 n^{\frac{1}{2}} .
$$

Hence (3.7.5) holds with $\tilde{c}=500$ if $d_{n} \geq 10^{-4}$. Assume therefore that $d_{n}<10^{-4}$. We distinguish two cases.

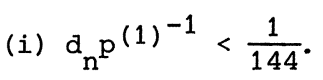

We now have

$$
\begin{aligned}
& I^{*}\left(p^{(1)}+3\left(d_{n} p^{(1)}\right)^{\frac{1}{2}}, p^{(1)}\right)= \\
& =\left\{p^{(1)}+3\left(d_{n} p^{(1)}\right)^{\frac{1}{2}}\right\} \log \left\{1+3 d_{n}^{\frac{1}{2}} p^{(1)^{-\frac{1}{2}}}\right\}+ \\
& +\left\{1-p^{(1)}-3\left(d_{n} p^{(1)}\right)^{\frac{1}{2}}\right\} \log \left\{1-3\left(1-p^{(1)}\right)^{-1}\left(d_{n} p^{(1)}\right)^{\frac{1}{2}}\right\} \\
& \geq\left\{p^{(1)}+3\left(d_{n} p^{(1)}\right)^{\frac{1}{2}}\right\}\left\{3 d_{n}^{\frac{1}{2}} p^{(1)^{-\frac{1}{2}}}-\frac{9}{2} d_{n} p^{\left.(1)^{-1}\right\}+}\right. \\
& +\left\{1-p^{(1)}-3\left(d_{n} p^{(1)}\right)^{\frac{1}{2}}\right\}\left\{-3\left(1-p^{(1)}\right)^{-1}\left(d_{n} p^{(1)}\right)^{\frac{1}{2}}-6 d_{n} p^{(1)}(1-p(1),-2\}\right. \\
& =\frac{9}{2} d_{n}-\frac{27}{2} d_{n}^{3 / 2} p^{(1)^{-\frac{1}{2}}}+3 d_{n} p^{(1)}(1-p(1))^{-1}-18\left(d_{n} p^{(1)}\right)^{3 / 2}\left(1-p^{(1)}\right)^{-2} \\
& \geq\left(\frac{9}{2}-\frac{27}{2} \cdot \frac{1}{12}\right) d_{n} \geq 3 d_{n}>d_{n},
\end{aligned}
$$

where the first inequality is established by the following consideration: 


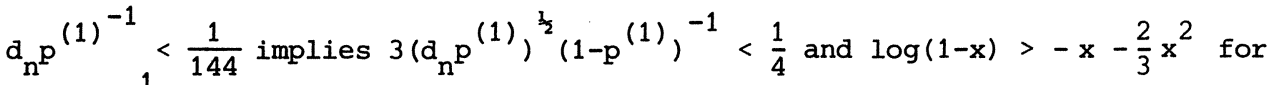
$0<x<\frac{1}{4}$. Similarly

$$
I^{*}\left(p^{(1)}-3\left(d_{n} p^{(1)}\right)^{\frac{1}{2}}, p^{(1)}\right)>d_{n} \text {. }
$$

Thus again using symmetry

$$
\begin{aligned}
& \sum_{j \in J_{1, n}} \operatorname{Pr}\left(x_{j n}=j\right) \leq \\
& \leq 1+n_{n\left[p^{(1)}-3\left(d_{n} p^{(1)}\right)^{\frac{1}{2}}\right]+1 \leq j \leq n\left[p^{(1)}+3\left(d_{n} p^{(1)}\right)^{\frac{1}{2}}\right]} \operatorname{Pr}\left(x_{j n}=j\right) \\
& \leq 1+2^{3 / 2} \int_{n\left[p^{(1)}-3\left(d_{n} p^{(1)}\right)^{\frac{3}{2}}\right]}^{n\left[3\left(d_{n} p^{(1)}\right)^{\frac{1}{2}}\right]} z^{-\frac{1}{2}} d z \leq 1+6 \cdot 2^{5 / 2}\left(n d_{n}\right)^{\frac{1}{2}}
\end{aligned}
$$

and hence (3.7.5) holds.

(ii) $d_{n} p^{(1)^{-1}} \geq \frac{1}{144}$.

In this case, since $500 d_{n}<\frac{1}{20}<\frac{1}{2}\left(1-p^{(1)}\right)$,

$$
\begin{aligned}
& I^{*}\left(p^{(1)}+500 d_{n}, p^{(1)}\right)= \\
& =\left(p^{(1)}+500 d_{n}\right) \log \left(1+500 d_{n} p^{(1)^{-1}}\right)+ \\
& +\left(1-p^{(1)}-500 d_{n}\right) \log \left\{1-500 d_{n}\left(1-p^{(1)}\right)^{-1}\right\} \\
& \geq\left(p^{(1)}+500 d_{n}\right) \log 4+\left(1-p^{(1)}-500 d_{n}\right)\left\{-500 d_{n}\left(1-p^{(1)}\right)^{-1}-\right. \\
& \\
& \left.500^{2} d_{n}^{2}\left(1-p^{(1)}\right)^{-2}\right\} \\
& \geq 500 d_{n}(\log 4-1)>d_{n} .
\end{aligned}
$$

This implies that

$$
\begin{aligned}
& \sum_{j \in J_{1, n}} \operatorname{Pr}\left(x_{j n}=j\right) \leq \sum_{0 \leq j \leq 644 n d_{n}} \operatorname{Pr}\left(x_{j n}=j\right) \\
& \leq 1+2^{\frac{1}{2}} \int_{0}^{644 n d_{n}} z^{-\frac{1}{2}} d z=1+\left(2^{3} \cdot 644\right)^{\frac{1}{2}}\left(n d_{n}\right)^{\frac{1}{2}}
\end{aligned}
$$


and therefore also in this case (3.7.5) holds.

This completes the proof of (3.7.2). Application of theorem 3.5.1 yields the theorem.

3.8. THE ALTERNATIVE HYPOTHESIS A PROPER SUBSET OF THE COMPLEMENT OF THE NULL HYPOTHESIS

In contrast with the remainder of this study in this section the testing problem

$$
\mathrm{H}_{0}: \theta \in \theta_{0} \text { against } \mathrm{H}_{1}: \theta \in \theta_{1}
$$

with level of significance $\alpha_{n}$ is considered, where $\theta_{1}$ is a proper subset of $\theta-\theta_{0}$. The LR test of this testing problem is given by

$$
\phi_{n}^{L R}(x)=\left\{\begin{array}{lll}
1 & > \\
\delta_{n} & \text { if } & L(x) \\
0 & & <
\end{array}\right.
$$

where

$$
L(x)=\sup _{\theta \in \Theta_{1} \cup \Theta_{0}}\left\{\theta^{\prime} x-\psi(\theta)\right\}-\sup _{\theta_{0} \epsilon \Theta_{0}}\left\{\theta_{0}^{\prime} x-\psi\left(\theta_{0}\right)\right\}
$$

and $d_{n}$ and $\delta_{n}$ are determined by

$$
\sup _{\theta_{0} \in \Theta_{0}}^{E} \theta_{0} \phi_{n}^{L R}\left(\bar{x}_{n}\right)=\alpha_{n}
$$

In this section the LR test of the testing problem

(**) $\mathrm{H}_{0}: \theta \in \theta_{0}$ against $\mathrm{H}_{1}^{*}: \theta \in \theta-\theta_{0}$

with level of significance $\alpha_{n}$ is denoted by

$$
\phi_{n}^{* L R}(x)=\left\{\begin{array}{llll}
1 & & > \\
\delta_{n}^{*} & \text { if } & L^{*}(x)=d^{*} \\
0 & & & <
\end{array}\right.
$$

where

$$
L^{*}(x)=\sup _{\theta \in \theta^{\prime}}\left\{\theta^{\prime} x-\psi(\theta)\right\}-\sup _{\theta_{0} \epsilon \Theta_{0}}\left\{\theta_{0}^{\prime} x-\psi\left(\theta_{0}\right)\right\}
$$

and $d_{n}^{*}$ and $\delta_{n}^{*}$ are determined by

$$
\sup _{\theta_{0} \epsilon \Theta_{0}}{ }_{\theta_{0}} \phi_{n}^{\star L R}\left(\bar{x}_{n}\right)=\alpha_{n} \text {. }
$$


In the previous sections several results were obtained concerning the shortcoming of the LR test of the testing problem $(* *)$. In many cases these results can be used to derive similarly properties of the LR test of the testing problem $(*)$. The (proof of the) following theorem gives an impression of this method.

THEOREM 3.8.1. Let $\theta_{0} \subset \mathrm{K} \subset$ int $\theta$, where $\mathrm{K}$ is a compact set, and let $\theta_{1}$ be an arbitrary subset of $\theta-\theta_{0}$. Consider the testing problem $\mathrm{H}_{0}: \theta \in \theta_{0}$ against $\mathrm{H}_{1}: \theta \in \theta_{1}$ with level of significance $\alpha_{n}$. Suppose $\alpha_{n} \geq \exp (-n I)$ for some $0<\mathrm{I}<\mathrm{I}\left(\theta_{0}\right)$, cf. (3.2.1). Let $\mathrm{M}$ be an arbitrary compact subset of int $\theta$. Then $\lim _{n \rightarrow \infty} R_{n}(\theta)=0$ uniformly on $M \wedge \theta_{1}$.

PROOF. By lemma 3.3.1 it holds that $\lim _{n \rightarrow \infty} n d_{n}^{*}=\infty$. Suppose that there exists a positive $\varepsilon$ and a sequence $\left\{\theta_{n}\right\}$ in $\theta_{1}$ satisfying $R_{n}\left(\theta_{n}\right) \geq \varepsilon$ and $\lim _{n \rightarrow \infty} \theta_{n}=\theta^{*} \epsilon$ int $\theta$. It will be shown that this leads to a contradiction and thus the result of the theorem is established.

Let $B_{n}=\left\{x ;\left\|x-\lambda\left(\theta_{n}\right)\right\| \leq c_{1} n^{-\frac{1}{2}}\right\}$ where the positive constant $c_{1}$ is so large that $P_{\theta_{n}}\left(\bar{x}_{n} \in B_{n}\right) \geq 1-\varepsilon / 4$. From now on let $n \geq n_{1}$, where $n_{1} \in \mathbb{N}$ is so large that $\lambda^{-1}\left(B_{n}\right) \subset$ int $\theta$ for all $n \geq n_{1}$. Hence for all $x \in B_{n}$ it holds that

$$
\begin{aligned}
L^{*}(x)-L(x) & \leq \lambda^{-1}(x)^{\prime} x-\psi\left(\lambda^{-1}(x)\right)-\theta_{n}^{\prime} x+\psi\left(\theta_{n}\right) \\
& =I\left(\lambda^{-1}(x), \theta_{n}\right) \leq c_{2} n^{-1}
\end{aligned}
$$

for some positive constant $\mathrm{c}_{2}$.

Define the sequence of tests $\left\{\phi_{n}^{* *}\right\}$ by

$$
\phi_{n}^{* *}(x)=\left\{\begin{array}{lll}
1 & & > \\
0 & \text { if } \quad L^{*}(x) & d_{n}^{*}+c_{2} n^{-1} .
\end{array}\right.
$$

Let $\left\{\vartheta_{n}\right\}$ be some sequence satisfying $I\left(\vartheta_{n}, \theta_{0}\right) \leq I+\delta$, where $\delta$ is a positive constant such that $I+\delta<I\left(\theta_{0}\right)$. In part (a) of the proof of theorem 3.3.2 it hàs been shown that

$$
\int\left[\chi_{n}^{+}(x)-\phi_{n}^{* L R}(x)\right] d \bar{P}_{\vartheta}^{-n}(x) \geq \varepsilon
$$

implies

$$
\alpha_{n} \geq \exp \left\{-n d_{n}^{*}+c_{3}\left(n d_{n}^{*}\right)^{\frac{1}{2}}\right\}
$$


for some positive constant $c_{3}$, where $x_{n}^{+}$is the size- $\alpha_{n}$ MP test of $H_{0}$ against $\theta=\vartheta_{n}$. Hence

implies

$$
\int\left[x_{n}^{+}(x)-\phi_{n}^{* *}(x)\right] d \bar{p}_{\vartheta_{n}}^{-n}(x) \geq \varepsilon
$$

$$
\alpha_{n} \geq \exp \left\{-n d_{n}^{*}+c_{4}\left(n d_{n}^{*}\right)^{\frac{1}{2}}\right\}
$$

for some positive constant $c_{4}$. By lemma 3.3 .3 it holds that

$$
\alpha_{n} \leq c_{5}\left(n d_{n}^{*}\right)^{(k-2) / 2} \exp \left(-n d_{n}^{*}\right)
$$

in contradiction to (3.8.2) for sufficiently large $n$, and thus the shortcoming of $\phi_{n}^{* *}$ tends to zero for such sequences $\left\{\vartheta_{n}\right\}$. Let $\left\{\tilde{\vartheta}_{n}\right\}$ be some sequence satisfying $I\left(\tilde{\vartheta}_{n}, \theta_{0}\right)>I+\delta$. It is easily seen that in this case the power of $\phi_{n}^{* *}$ tends to 1 (cf. part (b) of the proof of theorem 3.3.2). This implies that the shortcoming of $\phi_{n}^{* *}$ tends to zero uniformly over the whole set of alternatives.

Since

$$
\begin{aligned}
& \int\left[\phi_{n}^{+}(x)-\phi_{n}^{L R}(x)\right] d \bar{P}_{\theta_{n}^{n}}^{-n}(x)= \\
& =\int\left[\phi_{n}^{+}(x)-\phi_{n}^{* *}(x)\right] d \bar{P}_{\theta_{n}^{n}}^{-n}(x)+\int\left[\phi_{n}^{* *}(x)-\phi_{n}^{L R}(x)\right] d \bar{P}_{\theta_{n}^{n}}^{-n}(x) \\
& \leq \int\left[\phi_{n}^{+}(x)-\phi_{n}^{* *}(x)\right] d \bar{P}_{\theta_{n}^{n}}^{-n}(x)+P_{\theta_{n}}\left(\phi_{n}^{L R}\left(\bar{x}_{n}\right)<1, \phi_{n}^{* *}\left(\bar{x}_{n}\right)>0\right)
\end{aligned}
$$

and

$$
\int\left[\phi_{n}^{+}(x)-\phi_{n}^{* *}(x)\right] d P_{\theta}^{-n}(x) \leq \varepsilon / 2
$$

for all $n \geq n_{2}$, it follows that

$$
P_{\theta_{n}}\left(\phi_{n}^{L R}\left(\bar{x}_{n}\right)<1, \phi_{n}^{* *}\left(\bar{x}_{n}\right)>0\right) \geq \varepsilon / 2
$$

for all $n \geq n_{2}$. From now on let $n \geq n_{2}$. Using the definition of $B_{n}$ we obtain

$$
P_{\theta_{\mathrm{n}}}\left(\overline{\mathrm{x}}_{\mathrm{n}} \in \mathrm{B}_{\mathrm{n}}, \mathrm{L}\left(\overline{\mathrm{x}}_{\mathrm{n}}\right) \leq \mathrm{d}_{\mathrm{n}^{\prime}} \mathrm{L}^{*}\left(\overline{\mathrm{x}}_{\mathrm{n}}\right)>\mathrm{d}_{\mathrm{n}}^{*}+\mathrm{c}_{2} \mathrm{n}^{-1}\right) \geq \varepsilon / 4
$$

However, (3.8.1) implies that the set $\left\{x \in B_{n} ; L(x) \leq d_{n}, L^{*}(x)>d_{n}^{*}+c_{2} n^{-1}\right\}$ is empty and thus a contradiction is obtained. This completes the proof of the theorem. 
In BROWN (1971) it is suggested (heuristic principle 1) to forget "extra" information about the alternative hypothesis, implying the use of the LR test for a "larger" problem, obtained by imbedding $\theta_{0} \cup \theta_{1}$ in a larger parameter space, in lieu of $\phi_{n}^{L R}$. The reason for it is that extra information about the alternative can never increase the rate of exponential convergence to zero of the error probability of the second kind. However, forgetting this extra information can result in a decrease of power at other points of $\theta_{1}$ of subexponential and thus much larger order. This will be illustrated by the following example.

EXAMPLE 3.8.1. Let $\mathrm{x}_{1}, \mathrm{x}_{2}, \ldots$ be i.i.d. random variables with a normal $\mathrm{N}(\theta, 1)$ distribution. Consider the testing problem $\mathrm{H}_{0}: \theta=0$ against $\mathrm{H}_{1}: \theta \notin(-1,2)$ at level of significance $\alpha_{n}=\Phi\left(-n^{\frac{1}{2}}\right)+\Phi\left(-\frac{3}{2} n^{\frac{1}{2}}\right)$. The LR test of $H_{0}$ against $H_{1}$ rejects $\mathrm{H}_{5}$ if $\overline{\mathrm{x}}_{\mathrm{n}} \leq-1$ or $\overline{\mathrm{x}}_{\mathrm{n}} \geq \frac{3}{2}$, and thus its power at $\theta=-1$ equals $\frac{1}{2}+\Phi\left(-\frac{5}{2} n^{\frac{1}{2}}\right)$.

Brown's "larger" problem in this case is the testing problem $\mathrm{H}_{0}: \theta=0$ against $\mathrm{H}_{1}^{*}$ : $\theta \neq 0$. The $\mathrm{LR}$ test of $\mathrm{H}_{0}$ against $\mathrm{H}_{1}^{*}$ rejects $\mathrm{H}_{0}$ if

$$
\left|\bar{x}_{n}\right| \geq n^{-\frac{1}{2}} u_{1-\frac{1}{2} \alpha}
$$

where $u_{t}$ is defined by $\Phi\left(u_{t}\right)=t, 0<t<1$. By easy calculations it is found that its power at $\theta=-1$ equals $\frac{1}{2}-(2 \pi n)^{-\frac{1}{2}} \log 2+o\left(n^{-\frac{1}{2}}\right)$ as $n \rightarrow \infty$. Although this test has a faster rate of exponential convergence of the error probability of the second kind to zero at $\theta=2$ than the restricted LR test, this advantage is to be paid for by a decrease in power of order $n^{-\frac{1}{2}}$ at $\theta=-1$. 

CHAPTER IV

\section{RELATIONS BETWEEN SHORTCOMING \\ AND \\ BAHADUR DEFICIENCY}

\subsection{A FUNDAMENTAL THEOREM}

Shortcoming and Bahadur deficiency are tools to measure the performance of tests. Let $\left\{T_{n}\right\}$ be some sequence of tests; this sequence may be called optimal if the shortcoming of $T_{n}$ tends to zero for vanishing $\alpha_{n}$ ' the level of significance. The convergence can be pointwise or (stronger) uniform over (parts of) the parameter space. We have used this concept (in the uniform sense) in earlier chapters.

One can also call this sequence of tests optimal if the Bahadur deficiency of $\mathrm{T}_{\mathrm{n}}$ is small.

This chapter will be devoted to the relationship between these points of view.

Let $x_{1}, x_{2}, \ldots$ be i.i.d. random $k$-dimensional vectors with a distribution from an exponential family, i.e. $x_{1}$ has distribution $P_{\theta}$ satisfying

$$
\mathrm{dP}_{\theta}(\mathbf{x})=\exp \left\{\theta^{\prime} \mathbf{x}-\psi(\theta)\right\} \mathrm{d} \mu(\mathbf{x})
$$

Consider a family of tests $\left\{\phi_{n}^{\gamma} ; \gamma \in \Gamma\right\}, n=1,2, \ldots$. Here $\Gamma$ is an index set with the following interpretation: Let $\theta_{0}$ be some subset of $\theta$ (the null hypothesis) and let $0<\alpha<1$ (the level of significance); then there exists one and only one $\gamma \in \Gamma$, denoted by $\gamma_{n}(\alpha)$, such that

$$
\sup _{\theta_{0} \in \Theta_{0}} E_{\theta_{0}} \phi_{n}^{\gamma_{n}(\alpha)}\left(x_{1}, \ldots, x_{n}\right)=\alpha \text {. }
$$

As in section 3.5 assume that $\theta_{0}$ is a Borel set. For the testing problem $\mathrm{H}_{0}: \theta \in \theta_{0}$ against $\mathrm{H}_{1}: \theta \in \theta_{1}=\theta-\theta_{0}$ we have the following fundamental theorem: THEOREM 4.1.1. Let $\theta_{1} \in$ int $\theta_{1}$. The family of tests $\left\{\phi_{n}^{\gamma} ; \gamma \in \Gamma\right\}$ is deficient in the sense of Bahadur at $\theta_{1}$ of order $O\left(\mathrm{~N}^{+}\left(\alpha, \beta, \theta_{1}\right)^{\frac{1}{2}}\right)$ as $\alpha \rightarrow 0$ iff the short- 
coming of $\left\{\phi_{n}^{\gamma_{n}\left(\alpha_{n}\right)}\right\}$ at $\theta_{1}$ tends to zero as $n \rightarrow \infty$ for each sequence $\left\{\alpha_{n}\right\}$ satisfying $\lim _{\mathrm{n} \rightarrow \infty} \alpha_{\mathrm{n}}=0$.

REMARK 4.1.1. Roughly spoken the result of the theorem (and its proof) is essentially based on the fact that the asymptotical power $\beta(0<\beta<1)$ at an alternative $\theta_{1}$ of the MP test against $\theta_{1}$ increases iff the number of observations $\mathrm{n}$ is raised by at least $\delta \mathrm{n}^{\frac{1}{2}}(\delta>0)$.

Before proving theorem 4.1.1 we present a useful lemma about Euclidean distance and "Kullback-Leibler distance".

LEMMA 4.1.2. Let $\left\{\vartheta_{n}\right\}$ and $\left\{\theta_{n}\right\}$ be sequences in $\theta$. If $\lim _{n \rightarrow \infty} \vartheta_{n}=\vartheta \in$ int $\theta$ and $\lim _{n \rightarrow \infty}\left\|\vartheta_{n}-\theta_{n}\right\|=\infty$ then $\lim _{n \rightarrow \infty} I\left(\vartheta_{n}, \theta_{n}\right)=\infty$.

PROOF. By assumption $0 \in$ int $\theta$ implying $\left\{\theta ;\|\theta\| \leq c_{1}\right\} \subset$ int $\theta$ for some positive constant $c_{1}$. Hence for each subsequence there is a further subsequence, say $\left\{n_{i}\right\}$, such that

$$
c_{1}\left\|\theta_{n_{i}}{ }^{-\vartheta} n_{i}\right\|^{-1}\left(\theta_{n_{i}}{ }^{-\vartheta} n_{i}\right) \rightarrow \theta^{*} \epsilon \text { int } \theta
$$

It suffices to prove $\lim _{i \rightarrow \infty} I\left(\vartheta_{n_{i}}, \theta_{n_{i}}\right)=\infty$. Since $E_{\vartheta} \theta^{*} \cdot\left(x_{1}-\lambda(\vartheta)\right)=0$ and $P_{\vartheta}\left(\theta^{*},\left(x_{1}-\lambda(\vartheta)\right)=0\right)<1$ it follows that

$$
P_{\vartheta}\left(\theta^{*} \cdot\left(\mathrm{x}_{1}-\lambda(\vartheta)\right) \geq \varepsilon,\left\|\mathrm{x}_{1}-\lambda(\vartheta)\right\| \leq \mathrm{c}_{2}\right) \geq \delta
$$

for some positive constants $\varepsilon, \delta$ and $c_{2}$, and thus for all $i \geq i_{1}$

$$
P_{\vartheta_{i}}\left(\theta^{*}\left(\mathrm{x}_{1}-\lambda(\vartheta)\right) \geq \varepsilon,\left\|\mathrm{x}_{1}-\lambda(\vartheta)\right\| \leq \mathrm{c}_{2}\right) \geq \frac{1}{2} \delta
$$

Moreover, for all $i \geq i_{2}$,

$$
\begin{aligned}
& \left\{x_{i} \theta^{*},(x-\lambda(\vartheta)) \geq \varepsilon,\|x-\lambda(\vartheta)\| \leq c_{2}\right\} \\
& c\left\{x_{i} c_{1}\left\|\theta_{n_{i}}{ }^{-\vartheta} n_{i}\right\|^{-1}\left(\theta_{n_{i}}{ }^{-\vartheta} n_{i}\right) '\left(x-\lambda\left(\vartheta_{n_{i}}\right)\right) \geq \frac{1}{2} \varepsilon,\|x-\lambda(\vartheta)\| \leq c_{2}\right\}
\end{aligned}
$$

and thus, for all $i \geq \max \left(i_{1}, i_{2}\right)$,

$$
\begin{aligned}
& I\left(\vartheta_{n_{i}}, \theta_{n_{i}}\right)=\log \left[\int \exp \left\{\left(\theta_{n_{i}}{ }^{-\vartheta} n_{i}\right)^{\prime}\left(x-\lambda\left(\vartheta_{n_{i}}\right)\right)\right\} d P_{\vartheta_{n}}(x)\right] \\
& \geq \log \left[\frac{1}{2} \delta \exp \left\{\frac{1}{2} \varepsilon c_{1}^{-1}\left\|\theta_{n_{i}}{ }^{-\vartheta} n_{i}\right\|\right\}\right],
\end{aligned}
$$


which implies

$$
\lim _{i \rightarrow \infty} I\left(\vartheta_{n_{i}}, \theta_{n_{i}}\right)=\infty \text {. }
$$

This completes the proof of the lemma.

PROOF OF THEOREM 4.1.1. First assume that the family of tests $\left\{\phi_{n}^{\gamma} ; \gamma \in \Gamma\right\}$ is deficient in the sense of Bahadur at $\theta_{1}$ of order $\sigma^{\prime}\left(N^{+}\left(\alpha, \beta, \theta_{1}\right)^{\frac{1}{2}}\right)$ as $\alpha \rightarrow 0$.

Suppose there exists a sequence $\left\{\alpha_{n}\right\}, \alpha_{n} \rightarrow 0$, such that

$$
E_{\theta_{1}} \phi_{n}^{\gamma_{n}\left(\alpha_{n}\right)}\left(x_{1}, \ldots, x_{n}\right)-E_{\theta_{1}} \phi_{n}^{+, \alpha}{ }^{n}\left(\bar{x}_{n}\right)
$$

does not tend to zero. (Here $\phi_{\mathrm{n}}^{+, \alpha}$ denotes the size- $\alpha$ MP test of $\mathrm{H}_{0}: \theta_{0} \in \dot{\theta}_{0}$ against $\mathrm{H}_{1}^{*}: \theta=\theta_{1}$.) Then there exists a positive number $\varepsilon$ and a subsequence $\left\{\mathrm{n}_{i}\right\}$ such that

$$
E_{\theta_{1}} \phi_{n_{i}}^{+, \alpha_{n_{i}}}\left(\bar{x}_{n_{i}}\right)-E_{\theta_{1}} \phi_{n_{i}}^{\gamma_{n_{i}}}\left(\alpha_{n_{i}}\right)\left(x_{1}, \ldots, x_{n_{i}}\right) \geq \varepsilon \quad(i=1,2, \ldots) .
$$

Without loss of generality assume that $\lim _{i \rightarrow \infty} E_{\theta_{1}} \phi_{n_{i}}^{+, \alpha_{n_{i}}}\left(\bar{x}_{n_{i}}\right)=\beta_{0} \geq \varepsilon$. Let

and

$$
N\left(\alpha, \beta, \theta_{1}\right)=\inf \left\{n_{i} E_{\theta_{1}} \phi_{m}^{\gamma_{m}(\alpha)}\left(x_{1}, \ldots, x_{m}\right) \geq \beta, m \geq n\right\},
$$

then



$$
\mathrm{N}^{+}\left(\alpha_{\mathrm{n}_{i}}, \beta_{0}-\varepsilon / 4, \theta_{1}\right) \leq \mathrm{n}_{i} \leq \mathrm{N}\left(\alpha_{\mathrm{n}_{i}}, \beta_{0}-3 \varepsilon / 4, \theta_{1}\right)
$$

for $i \geq i_{0}$. From now on let $i \geq i_{0}$.

Let $\mathrm{N}_{i}=\mathrm{N}^{+}\left(\alpha_{\mathrm{n}_{i}}, \beta_{0}-3 \varepsilon / 4, \theta_{1}\right)-1$. There exists a sequence $\left\{\delta_{i}\right\}$ satisfying $\lim _{i \rightarrow \infty} \delta_{i}=0^{i}$ and

$$
(4.1,1) \quad \mathrm{N}\left(\alpha_{\mathrm{n}_{i}}, \beta_{0}-3 \varepsilon / 4, \theta_{1}\right) \leq \mathrm{N}_{i}+1+\delta_{i}\left(\mathrm{~N}_{i}+1\right)^{\frac{1}{2}} \text {. }
$$

Hence we obtain

$$
\text { (4.1.2) } \quad \mathrm{N}^{+}\left(\alpha_{\mathrm{n}_{i}}, \beta_{0}-\varepsilon / 4, \theta_{1}\right) \leq \mathrm{N}_{i}+1+\delta_{i}\left(\mathrm{~N}_{i}+1\right)^{\frac{1}{2}}
$$

and we therefore restrict our attention to MP tests. Let 
80

$$
N_{i}^{\star}=N_{i}+1+\delta_{i}\left(N_{i}+1\right)^{\frac{1}{2}}
$$

The test

$$
\mathrm{N}_{\mathrm{i}}^{+, \alpha_{\mathrm{n}}}
$$

has the following form:

$$
{ }_{N_{i}^{*}}^{+, \alpha}{ }^{*}(x)=\left\{\begin{array}{lc}
1 & x \in E \\
\text { if } & N_{i}^{*} \\
0 & x \notin F \\
N_{i}^{*}
\end{array},\right.
$$

where

$$
E_{N_{i}^{*}}^{*}=\left\{x_{i} \lim \inf \int_{j \rightarrow \infty} \exp \left\{N_{i}^{*}\left(\theta_{0}-\theta_{1}\right) ' x-N_{i}^{*} \psi\left(\theta_{0}\right)+N_{i}^{*} \psi\left(\theta_{1}\right)\right\} d \lambda_{j, i}\left(\theta_{0}\right)<1\right\}
$$

and

$$
F_{N_{i}^{*}}=\left\{x_{i} \lim \sup \iint_{\theta_{0} \rightarrow \infty} \exp \left\{N_{i}^{*}\left(\theta_{0}-\theta_{1}\right) ' x-N_{i}^{*} \psi\left(\theta_{0}\right)+N_{i}^{*} \psi\left(\theta_{1}\right)\right\} d \lambda \lambda_{j, i}\left(\theta_{0}\right) \leq 1\right\}
$$

(cf. (3.5.2)).

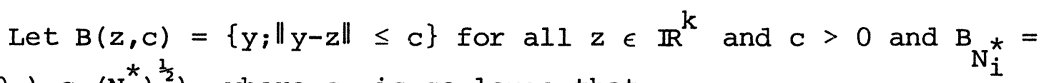
$B\left(\lambda\left(\theta_{1}\right), c_{1}\left(N_{i}^{*}\right)^{\frac{1}{2}}\right)$, where $c_{1}$ is so large that

(4.1.3) $\quad P_{\theta_{1}}\left(\overline{\mathrm{x}}_{\mathrm{N}_{i}^{*}}^{*} \underset{\mathrm{N}_{i}^{*}}{*}\right)<\varepsilon / 10$.

Since $\mathrm{F}_{\mathrm{N}_{\mathrm{i}}^{*}}$ is a convex set we can choose $\mathrm{c}_{2}>0$ so small that

$$
\mathrm{Q}_{\mathrm{N}_{i}}=\left\{\mathrm{y} ; \mathrm{B}\left(\mathrm{y}, \mathrm{c}_{2} \mathrm{~N}_{\mathrm{i}}^{-\frac{1}{2}}\right) \subset \underset{\mathrm{N}_{i}^{*}}{*} \stackrel{\mathrm{B}_{\mathrm{i}}^{*}}{\mathrm{~N}_{\mathrm{i}}^{*}}\right\}
$$

satisfies

(4.1.4) $\quad P_{\theta_{1}}\left(\overline{\mathrm{X}}_{\mathrm{N}_{i}} \in \underset{\mathrm{N}_{i}^{*}}{*} \wedge \mathrm{B}_{i}^{*} \wedge \mathrm{Q}_{\mathrm{N}_{i}}^{\mathrm{C}}\right) \leq \varepsilon / 10$.

Define the test $\phi_{\mathrm{N}_{i}}$ by 


$$
\phi_{N_{i}}(x)=\left\{\begin{array}{lll}
1 & \epsilon \\
& \text { if } x & \rho_{N_{i}} \\
0 & \notin
\end{array} .\right.
$$

We shall prove that

$$
\text { (4.1.5) } \quad \mathrm{E}_{\theta_{1}} \phi_{\mathrm{N}_{i}}\left(\overline{\mathrm{X}}_{\mathrm{N}_{i}}\right)>\beta_{0}-3 \varepsilon / 4,
$$

and

$$
\text { (4.1.6) } \sup _{\theta_{0} \epsilon \theta_{0}} \mathrm{E}_{\theta_{0}} \phi_{\mathrm{N}_{i}}\left(\overline{\mathrm{x}}_{\mathrm{N}_{i}}\right) \leq \alpha_{\mathrm{n}_{i}}
$$

for $i$ sufficiently large.

If $A$ is some set in $\mathbb{R}^{k}, b \in \mathbb{R}^{k}$ and $t \neq 0 \in \mathbb{R}^{1}$, denote by $(A-b) t=\left\{x \in \mathbb{R}^{k} ; t^{-1} x+b \in A\right\}$. Let $U$ be multivariate normally $N\left(0 ; \Sigma_{\theta_{1}}\right)$ distributed, then the following (in)equalities hold for $i$ sufficiently large:

$$
\begin{aligned}
& \beta_{0}-\varepsilon / 4<P_{\theta_{1}}\left(\bar{X}_{N_{i}^{*}} \in F_{N_{i}^{*}} \wedge B_{i}^{*}\right)+\varepsilon / 10 \\
& \leq \operatorname{Pr}\left(U \in\left\{\mathrm{N}_{i}^{*} \wedge \mathrm{B}_{N_{i}^{*}}^{*}-\lambda\left(\theta_{1}\right)\right\}\left(\mathrm{N}_{i}^{*}\right)^{\frac{1}{2}}\right)+2 \varepsilon / 10 \\
& \leq \operatorname{Pr}\left(U \in\left\{\mathrm{N}_{i}^{*} \wedge \mathrm{B}_{i}^{*}-\lambda\left(\theta_{1}\right)\right\} \mathrm{N}_{\mathrm{i}}^{\frac{1}{2}}\right)+3 \varepsilon / 10 \\
& \leq P_{\theta_{1}}\left(\overline{\mathrm{x}}_{\mathrm{N}_{i}} \in \mathrm{F}_{\mathrm{N}_{\mathrm{i}}^{*}} \wedge \mathrm{B}_{\mathrm{i}}^{\star}\right)+4 \varepsilon / 10 \\
& \leq P_{\theta_{1}}\left(\overline{\mathrm{x}}_{\mathrm{N}_{\mathrm{i}}} \in \mathrm{Q}_{\mathrm{N}_{\mathrm{i}}}\right)+5 \varepsilon / 10 \\
& =\mathrm{E}_{\theta_{1}} \phi_{\mathrm{N}_{\mathrm{i}}}\left(\overline{\mathrm{x}}_{\mathrm{N}_{i}}\right)+\varepsilon / 2 \text {, }
\end{aligned}
$$

which completes the proof of (4.1.5).

Let $\theta_{0} \in \theta_{0}$, and $i$ so large that $Q_{N_{i}} \subset \Lambda$.

Since $Q_{\mathrm{N}_{i}}$ is a closed set, there exists $\vartheta \in \lambda^{-1}\left(Q_{\mathrm{N}_{i}}\right)$ satisfying $I\left(\vartheta, \theta_{0}\right)=$ $I\left(\lambda^{-1}\left(Q_{N_{i}}\right), \theta_{0}\right)$. By lemma 3.4 .3 the convexity of $\Omega_{N_{i}}$ implies

$$
\left(\vartheta-\theta_{0}\right) \cdot x-\psi(\vartheta)+\psi\left(\theta_{0}\right) \geq I\left(\vartheta, \theta_{0}\right)
$$

for all $x \in Q_{N_{i}}$. Hence

(4.1.7) $\quad P_{\theta_{0}}\left(\overline{\mathrm{x}}_{\mathrm{N}_{i}} \in \mathrm{Q}_{\mathrm{N}_{\mathrm{i}}}\right) \leq P_{\theta_{0}}\left(\left(\vartheta-\theta_{0}\right) \cdot\left(\overline{\mathrm{x}}_{\mathrm{N}_{i}}-\lambda(\vartheta)\right) \geq 0\right) \leq$ 
82

$$
\begin{aligned}
& \leq \int_{\left(\vartheta-\theta_{0}\right)^{\prime}(x-\lambda(\vartheta)) \geq 0} \exp \left\{-\mathrm{N}_{i}\left(\vartheta-\theta_{0}\right) \mathrm{x}^{-\mathrm{N}_{i}} \psi\left(\theta_{0}\right)+\mathrm{N}_{i} \psi(\vartheta)\right\} \mathrm{d}_{\vartheta}^{\mathrm{N}_{i}}(\mathrm{x}) \\
& \leq \exp \left\{-\mathrm{N}_{i} I\left(\vartheta, \theta_{0}\right)\right\} \\
& =\exp \left\{-\mathrm{N}_{i} I\left(\lambda^{-1}\left(\mathrm{Q}_{\mathrm{N}_{i}}\right), \theta_{0}\right)\right\}
\end{aligned}
$$

for all $\theta_{0} \in \theta_{0}$. Let $\theta_{0 N_{i}} \in \theta_{0}$ be such that

(4.1.8) $\quad I\left(\lambda^{-1}\left(Q_{N_{i}}\right), \theta_{O_{N}}\right) \leq I\left(\lambda^{-1}\left(Q_{N_{i}}\right), \theta_{0}\right)+N_{i}^{-1}$.

Then it follows that

(4.1.9) $\sup _{\theta_{0} \in \Theta_{0}} E_{\theta_{0}} \phi_{N_{i}}\left(\bar{x}_{N_{i}}\right) \leq \exp \left\{-N_{i} I\left(\lambda^{-1}\left(Q_{N_{i}}\right), \theta_{0 N_{i}}\right)+1\right\}$.

Let $\vartheta_{N_{i}} \in \lambda^{-1}\left(Q_{N_{i}}\right)$ satisfy $I\left(\vartheta_{N_{i}}, \theta_{O N_{i}}\right)=I\left(\lambda^{-1}\left(Q_{N_{i}}\right), \theta_{0 N_{i}}\right)$. By definition of $Q_{N_{i}}$ there exists ${ }^{i}$ a sphere with ${ }^{i}$ entre $\vartheta_{N_{i}}$ and radius $c_{3} N_{i}^{-\frac{1}{2}}$ contained in $\lambda^{-1}\left(F_{N_{i}^{*}} \wedge B_{N_{i}^{*}}\right)$. Defining $n_{N_{i}}$ by $n_{N_{i}}=\vartheta_{N_{i}}+\frac{1}{2} C_{3} N_{i}^{-\frac{1}{2}}\left\|\theta_{0 N_{i}}{ }^{-\vartheta} N_{i}\right\|^{-1}\left(\theta_{O N_{i}} \vartheta_{N_{i}}\right)$ there exist positive constants $c_{4}$ and $c_{5}$ such that $B\left(\lambda\left(n_{N_{i}}\right), c_{4}\left(N_{i}^{*}\right)^{-\frac{1}{2}}\right)^{i} c$ $\mathrm{F}_{\mathrm{N}_{1}^{*}} \wedge \mathrm{B}_{\mathrm{N}_{\mathrm{i}}^{*}}$ and

$$
\begin{aligned}
& \text { (4.1.10) } I\left(n_{N_{i}}, \theta_{O_{N}}\right)=I\left(\vartheta_{N_{i}}, \theta_{O_{N}}\right)+I\left(n_{N_{i}}, \vartheta_{N_{i}}\right)+\left(\vartheta_{N_{i}}-\theta_{0 N_{i}}\right) \cdot\left\{\lambda\left(n_{N_{i}}\right)-\lambda\left(\vartheta_{N_{i}}\right)\right\} \\
& \leq I\left(\vartheta_{N_{i}}, \theta_{O N_{i}}\right)-c_{5}\left(N_{i}^{*}\right)^{-\frac{1}{2}}
\end{aligned}
$$

for i sufficiently large.

Since

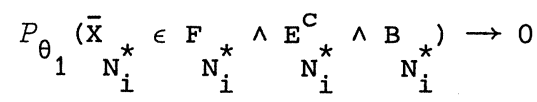

as $i \rightarrow \infty$ (cf. the proof of theorem 3.5.1) and $\left\|n_{N_{i}}-\theta_{1}\right\| \leq c_{6}\left(N_{i}^{*}\right)^{-\frac{1}{2}}$ for some positive constant $c_{6}$, it is easily seen that also

$$
\lim _{i \rightarrow \infty} P_{\eta_{N_{i}}}\left(\bar{x}_{N_{i}^{*}} \in F_{N_{i}^{*}}^{*} \wedge E_{N_{i}^{*}}^{{ }^{c}} \wedge B_{N_{i}^{*}}^{*}\right)=0 .
$$

Hence for $i$ sufficiently large 
(4.1.11) $\alpha_{n_{i}} \geq \int_{N_{i}^{*}}^{+, \alpha_{n}}{ }_{i}^{*}(x) \exp \left\{N_{i}^{*}\left(\theta_{0 N_{i}}-n_{N_{i}}\right) ' x-N_{i}^{*} \psi\left(\theta 0 N_{i}\right)+N_{i}^{*} \psi\left(n_{N_{i}}\right)\right\} d \bar{P}_{i}^{-N_{i}^{*}}(x)$
$\left\{x \in B\left(\lambda\left(n_{N_{i}}\right), c_{4}\left(N_{i}^{*}\right)^{-\frac{1}{2}}\right) ;\left(\theta_{O N_{i}}-n_{N_{i}}\right)^{\prime}\left(x-\lambda\left(n_{N_{i}}\right)\right) \geq 0\right\}$

$$
\begin{aligned}
& \geq c_{7} \exp \left\{-\mathrm{N}_{\mathrm{i}}^{*} \mathrm{I}\left(\eta_{\mathrm{N}_{\mathrm{i}}},{ }{ }_{\mathrm{ON}}\right)\right\} \\
& \geq c_{7} \exp \left\{-\mathrm{N}_{i}^{*} I\left(\vartheta_{\mathrm{N}_{\mathrm{i}}}{ } \theta_{\mathrm{ON}_{\mathrm{i}}}\right)+\mathrm{c}_{5}\left(\mathrm{~N}_{\mathrm{i}}^{*}\right)^{\frac{1}{2}}\right\} \\
& \geq \exp \left\{-\mathrm{N}_{\mathrm{i}} \mathrm{I}\left(\vartheta_{\mathrm{N}_{\mathrm{i}}}, \theta_{\mathrm{ON}_{\mathrm{i}}}\right)+1\right\} \\
& =\exp \left\{-\mathrm{N}_{i} I\left(\lambda^{-1}\left(Q_{\mathrm{N}_{i}}\right), \theta_{0 \mathrm{~N}_{i}}\right)+1\right\} \text {, }
\end{aligned}
$$

where $c_{7}$ is a positive constant. Combining (4.1.9) and (4.1.11) we obtain $(4.1 .6)$. By $(4.1 .5)$ and $(4.1 .6)$ it follows that

$$
\mathrm{N}_{i}^{+}\left(\alpha_{n_{i}}, \beta_{0}-3 \varepsilon / 4, \theta_{1}\right) \leq \mathrm{N}_{i}
$$

in contradiction to the definition of $\mathrm{N}_{i}$. This completes the first part of the proof.

Now assume that the shortcoming of $\left\{\phi_{n}^{\gamma} ; \gamma \in \Gamma\right\}$ at $\theta_{1}$ tends to zero as $n \rightarrow \infty$ for each sequence $\left\{\alpha_{n}\right\}$ satisfying $\lim _{n \rightarrow \infty} \alpha_{n}=0$.

Suppose there exist a positive number $\varepsilon_{0}$, a number $\beta_{1} \in(0,1)$ and a sequence $\left\{\alpha_{i}\right\}$ tending to zero satisfying

$$
N\left(\alpha_{i}, \beta_{1}, \theta_{1}\right)>\mathbf{N}^{+}\left(\alpha_{i}, \beta_{1}, \theta_{1}\right)+\varepsilon_{0}\left\{\mathrm{~N}^{+}\left(\alpha_{i}, \beta_{1}, \theta_{1}\right)\right\}^{\frac{3}{2}}
$$

Let $M_{i}=N^{+}\left(\alpha_{i}, \beta_{1}, \theta_{1}\right)$ and $M_{i}^{*}=\operatorname{entier}\left\{M_{i}+\varepsilon_{0} M_{i}^{\frac{1}{2}}\right\}$. The size- $\alpha_{i}$ MP test of $\mathrm{H}_{0}: \theta \in \theta_{0}$ against $\mathrm{H}_{1}^{*}: \theta=\theta_{1}$ has the following form:

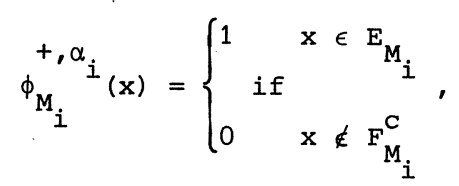

where $\mathrm{F}_{\mathrm{M}_{i}}$ is a convex set and $P_{\theta_{1}}\left(\overline{\mathrm{X}}_{\mathrm{M}_{i}} \in \mathrm{E}_{\mathrm{M}_{i}}^{\mathrm{C}} \wedge \mathrm{F}_{\mathrm{M}_{i}}\right) \rightarrow 0$ as $\mathrm{M}_{i} \rightarrow \infty$.

Since $\theta_{1} \in$ int $\theta_{1}$ there exists a positive constant $c_{8}$ such that $B\left(\theta_{1}, c_{8}\right) \subset$ int $\theta_{1}$. Fix a point $\bar{\theta}_{0} \epsilon$ int $\theta_{0}$. Then by lemma 4.1 .2 
$(4.1 .13) \quad c_{9}=\sup \left\{\left\|\vartheta-\theta_{0}\right\| ; \vartheta \in B\left(\theta_{1}, c_{8}\right), \theta_{0} \in \theta_{0}, I\left(\vartheta, \theta_{0}\right) \leq I\left(\theta_{1}, \bar{\theta}_{0}\right)+1\right\}$

is a finite number. Since $I\left(\tilde{\vartheta}, \theta_{0}\right)-I\left(\vartheta, \theta_{0}\right)=I(\tilde{\vartheta}, \vartheta)+\left(\vartheta-\theta_{0}\right) \cdot\{\lambda(\tilde{\vartheta})-\lambda(\vartheta)\}$ it follows by $(4.1 .13)$ that

$c_{10}=\sup \left\{\frac{I\left(\tilde{\vartheta}, \theta_{0}\right)-I\left(\vartheta, \theta_{0}\right)}{\|\lambda(\tilde{\vartheta})-\lambda(\vartheta)\|} ; \vartheta, \tilde{\vartheta} \in B\left(\theta_{1}, c_{8}\right), \vartheta \neq \tilde{\vartheta}, \theta_{0} \in \theta_{0}, I\left(\vartheta, \theta_{0}\right) \leq I\left(\theta_{1}, \bar{\theta}_{0}\right)+1\right\}$

is also a finite number. Let $\tau$ be defined by

$$
\text { (4.1.14) } \tau=c_{10}^{-1}\left(\varepsilon_{0} / 8\right) \inf \left\{I\left(\vartheta, \Theta_{0}\right) ; \vartheta \in B\left(\theta_{1}, c_{8}\right)\right\}
$$

Consider the sphere $B\left(\lambda\left(\theta_{1}\right), c_{11}{ }_{i}^{-\frac{1}{2}}\right)$, where $c_{11}$ is so large that $F_{M_{i}}^{C} \wedge B\left(\lambda\left(\theta_{1}\right), C_{1}\left(M_{i}^{*}\right)^{-\frac{1}{2}}\right)$ contains a sphere with radius $\tau\left(M_{i}^{*}\right)^{-\frac{1}{2}}$. Denoting by $\mathrm{U}$ a k-variate normally $\mathrm{N}\left(0 ; \Sigma_{\theta_{1}}\right)$ distributed random vector we define $\tau^{*}$ by

$$
\tau^{*}=\inf \left\{\operatorname{Pr}(U \in \tilde{B}) ; \tilde{B} \text { a sphere with radius } \tau, \tilde{B} \subset B\left(0, c_{11}\right)\right\} \text {. }
$$

Let $c_{12}>c_{11}$ be so large that $P_{\theta_{1}}\left(\bar{X}_{M_{i}} \notin B\left(\lambda\left(\theta_{1}\right), c_{12} M_{i}^{-\frac{1}{2}}\right)\right) \leq \tau^{*} / 6$, and let

$$
\mathrm{G}_{i}^{*}=\left\{\mathrm{y} ; \mathrm{B}\left(\mathrm{y} ; \tilde{\tau}\left(\mathrm{M}_{i}^{*}\right)^{-\frac{1}{2}}\right) \subset \mathrm{F}_{M_{i}} \wedge B\left(\lambda\left(\theta_{1}\right), \mathrm{C}_{12} \mathrm{M}_{i}^{-\frac{1}{2}}\right)\right\},
$$

where $\tilde{\tau}$ is so small that

$$
P_{\theta_{1}}\left(\overline{\mathrm{X}}_{\mathrm{M}_{i}} \in \mathrm{F}_{\mathrm{M}_{i}} \wedge \mathrm{B}\left(\lambda\left(\theta_{1}\right), \mathrm{C}_{12} \mathrm{M}_{i}^{-\frac{1}{2}}\right) \wedge \underset{\mathrm{M}_{i}^{*}}{{ }^{\mathrm{C}}}\right) \leq \tau^{*} / 6
$$

By definition of $C_{11}$ there exists a point $\lambda\left(\xi_{i}\right)$ satisfying int $B\left(\lambda\left(\xi_{j}\right)\right.$, $\tau\left(M_{i}^{*}\right)^{-\frac{1}{2}} \subset G_{M_{i}^{*}}^{C} \wedge B\left(\lambda\left(\theta_{1}\right), C_{11}\left(M_{i}^{*}\right)^{-\frac{1}{2}}\right)$, and $B\left(\lambda\left(\xi_{i}\right), \tau\left(M_{i}^{*}\right)^{-\frac{1}{2}}\right) \wedge G_{M_{i}^{*}} \neq \varnothing$.

Define the test $\phi_{M_{i}^{*}}$ by

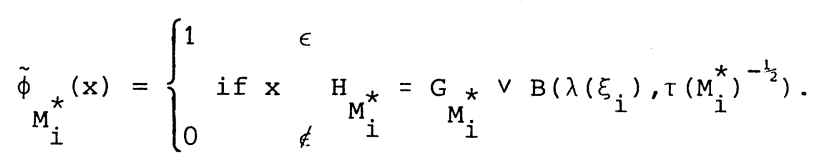

We shall prove that



and

$$
\text { (4.1.16) } \left.\sup _{\theta_{0} \in \Theta_{0}} \mathrm{E}_{\theta_{0}} \tilde{\phi}_{i}^{\mathrm{\phi}_{i}^{*}} \mathrm{M}_{i}^{*}\right) \leq \alpha_{i}
$$


for $i$ sufficiently large.

The following (in)equalities hold for $i$ sufficiently large:

$$
\begin{aligned}
& E_{\theta_{1}} \tilde{\phi}_{i}^{{ }^{*}}{ }_{M_{i}^{*}}{ }^{*} \geq \\
& \geq \operatorname{Pr}\left(U \in\left\{\mathrm{M}_{i}^{*}-\lambda\left(\theta_{1}\right)\right\}\left(M_{i}^{*}\right)^{\frac{1}{2}}\right)-\tau^{*} / 6 \\
& =\operatorname{Pr}\left(U \in\left\{B\left(\lambda\left(\xi_{i}\right), \tau\left(M_{i}^{*}\right)^{-\frac{1}{2}}\right)-\lambda\left(\theta_{1}\right)\right\}\left(M_{i}^{*}\right)^{\frac{1}{2}}\right)+ \\
& \operatorname{Pr}\left(U \in \left\{\mathrm{M}_{i}^{*}{ }^{\left.\left.-\lambda\left(\theta_{1}\right)\right\}\left(M_{i}^{*}\right)^{\frac{1}{2}}\right)-\tau^{*} / 6}\right.\right. \\
& \geq \operatorname{Pr}\left(U \in \left\{\mathrm{M}_{i}^{*}{ }^{\left.\left.-\lambda\left(\theta_{1}\right)\right\} M_{i}^{\frac{1}{2}}\right)}+4 \tau^{*} / 6\right.\right. \\
& \geq P_{\theta_{1}}\left(\overline{\mathrm{X}}_{\mathrm{M}_{\mathrm{i}}} \in \underset{\mathrm{M}_{i}^{*}}{*}\right)+3 \tau^{*} / 6 \\
& \geq P_{\theta_{1}}\left(\overline{\mathrm{X}}_{\mathrm{M}_{i}} \in \mathrm{F}_{\mathrm{M}_{i}} \wedge \mathrm{B}\left(\lambda\left(\theta_{1}\right), \mathrm{c}_{12} \mathrm{M}_{i}^{-\frac{1}{2}}\right)\right)+2 \tau^{*} / 6 \\
& \geq P_{\theta_{1}}\left(\overline{\mathrm{x}}_{\mathrm{M}_{i}} \in \mathrm{F}_{\mathrm{M}_{i}}\right)+\tau^{*} / 6 \\
& \geq \beta_{1}+\tau^{*} / 6 \text {, }
\end{aligned}
$$

establishing (4.1.15).

Let $\mathrm{CO}\left(\mathrm{H}_{\mathrm{M}_{\dot{j}}^{*}}\right)$ be the convex hull of $\mathrm{H}_{\mathrm{M}_{\dot{i}}^{*}}$. By convexity arguments (cf. (4.1.7)) ${ }^{M_{\dot{1}}^{*}}$ follows that

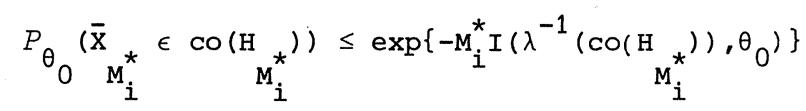

for all $\theta_{0} \in \theta_{0}$. Let $\theta_{0 i}^{*} \in \theta_{0}$ be such that

$$
\text { (4.1.17) } \left.I\left(\lambda^{-1}\left(\underset{M_{i}^{*}}{\left(\operatorname { c o } \left(\mathrm{H}_{0 i}\right.\right.}\right)\right), \theta_{0 i}^{*}\right) \leq I\left(\lambda^{-1}\left(\operatorname{co}\left(\mathrm{H}_{i}^{*}\right)\right), \theta_{0}\right)+\left(M_{i}^{*}\right)^{-1} \text {, }
$$

and let $\vartheta_{i}^{*} \in \lambda^{-1}\left(\mathrm{Co}\left(\mathrm{H}_{\mathrm{M}^{*}}\right)\right)$ satisfy $I\left(\vartheta_{i}^{*}, \theta_{0 i}^{*}\right)=I\left(\lambda^{-1}\left(\mathrm{Co}\left(\mathrm{H}_{\mathrm{M}_{i}^{*}}\right)\right), \theta_{0 i}^{*}\right)$.
Then it follows that

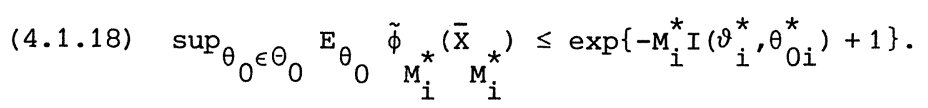

By definition of $\mathrm{H}_{\mathrm{M}_{i}^{*}}^{*}$ there exists a point $\lambda\left(\tilde{\vartheta}_{i}\right) \in \mathrm{M}_{i}^{{ }^{\star}}$ satisfying 
86

$$
\left\|\lambda\left(\tilde{\vartheta}_{i}\right)-\lambda\left(\vartheta_{i}^{*}\right)\right\| \leq 2 \tau\left(M_{i}^{*}\right)^{-\frac{1}{2}}
$$

Hence

$$
\begin{aligned}
& \left.\alpha_{i} \geq \int_{\left(\theta_{0 i}^{*}-\vartheta_{i}\right){ }^{\prime}\left(x-\lambda\left(\tilde{\vartheta}_{i}\right)\right) \geq 0}^{+, \alpha_{i}} \underset{M_{i}}{\exp \left\{M_{i}\right.}\left(\theta_{0 i}^{*}-\tilde{\vartheta}_{i}\right)^{\prime} x-M_{i} \psi\left(\theta_{0 i}^{*}\right)+M_{i} \psi\left(\tilde{\vartheta}_{i}\right)\right\} d \bar{p}_{\tilde{\vartheta}_{i}}^{M_{i}}(x) \\
& \geq \exp \left\{-M_{i} I\left(\tilde{\vartheta}_{i}, \theta_{0 i}^{*}\right)\right\} \times \\
& \times \int \quad B\left(\lambda\left(\tilde{\vartheta}_{i}\right), \tilde{\tau}\left(M_{i}^{*}\right)^{-\frac{1}{2}}\right) \wedge\left(\theta_{0 i}^{*}-\vartheta_{i}\right) \prime\left(x-\lambda\left(\tilde{\vartheta}_{i}\right)\right) \geq 0{ }^{\phi_{M_{i}}{ }^{+, \alpha}(x) d \bar{P}_{\tilde{\vartheta}_{i}}^{M_{i}}(x) .}
\end{aligned}
$$

Since $P_{\theta_{1}}\left(\overline{\mathrm{X}}_{\mathrm{M}_{i}} \in \mathrm{F}_{\mathrm{M}_{i}} \wedge \mathrm{E}_{\mathrm{M}_{i}}^{\mathrm{C}}\right) \rightarrow 0$ as $i \rightarrow \infty$ and $\left\|\lambda\left(\tilde{\vartheta}_{i}\right)-\lambda\left(\theta_{1}\right)\right\| \leq \mathrm{c}_{12} \mathrm{M}_{i}^{-\frac{1}{2}}$ it follows that $\lim _{i \rightarrow \infty} P_{\vartheta_{i}}\left(\bar{x}_{M_{i}} \in F_{M_{i}} \wedge E_{M_{i}}^{C}\right)=0$. Moreover $B\left(\lambda\left(\tilde{\vartheta}_{i}\right), \tilde{\tau}\left(M_{i}^{*}\right)^{-\frac{1}{2}}\right) \subset F_{M_{i}}$ and hence there exists a positive constant $c_{13}$ such that

$(4.1 .19) \quad \alpha_{i} \geq c_{13} \exp \left\{-M_{i} I\left(\vartheta_{i}, \theta_{0 i}^{*}\right)\right\}$

on the other hand for $i$ sufficiently large

$$
\begin{aligned}
& \exp \left\{-M_{i}^{*} I\left(\vartheta_{i}, \theta_{O i}^{*}\right)+1\right\} \leq \\
& \leq \exp \left\{-M_{i}^{*}\left[I\left(\tilde{\vartheta}_{i}, \theta_{O i}^{*}\right)-2 c_{10} \tau\left(M_{i}^{*}\right)^{-\frac{1}{2}}\right]+1\right\} \\
& \leq \exp \left\{-M_{i} I\left(\tilde{\vartheta}_{i}, \theta_{O i}^{*}\right)-\frac{1}{2} \varepsilon{ }_{0} M_{i}^{\frac{1}{2}} I\left(\tilde{\vartheta}_{i}, \theta_{O i}^{*}\right)+3 c_{10} \tau M_{i}^{\frac{1}{2}}\right\} \\
& \leq \exp \left\{-M_{i} I\left(\tilde{\vartheta}_{i}, \theta_{O i}^{*}\right)-4 c_{10} \tau M_{i}^{\frac{1}{2}}+3 c_{10} \tau M_{i}^{\frac{1}{2}}\right\} \\
& \leq c_{13} \exp \left\{-M_{i} I\left(\tilde{\vartheta}_{i}, \theta_{O i}^{*}\right)\right\},
\end{aligned}
$$

and hence, in combination with $(4.1 .18)$ and (4.1.19)

$$
\left.\sup _{\theta_{0} \in \theta_{0}} E_{\theta_{0}} \tilde{\phi}_{M_{i}^{*}}^{*} \bar{M}_{i}^{*}\right) \leq \alpha_{i}
$$

for $i$ sufficiently large, which completes the proof of (4.1.16).

Let $\tilde{M}_{i}=N\left(\alpha_{i}, \beta_{1}, \theta_{1}\right)-1$, then by (4.1.12) $\tilde{M}_{i} \geq M_{i}^{*}$ and hence in view of (4.1.15) and (4.1.16) it follows that

$$
E_{\theta_{1}} \phi_{\tilde{M}_{i}}^{+, \alpha}\left(\bar{X}_{\tilde{M}_{i}}\right) \geq \beta_{1}+\tau^{*} / 6
$$


Since the shortcoming of $\left\{\phi_{n}^{\gamma} ; \gamma \in \Gamma\right\}$ at $\theta_{1}$ tends to zero this implies

$$
E_{\theta_{1}} \phi_{\tilde{M}_{i}}^{\gamma_{\tilde{M}_{i}}\left(\alpha_{i}\right)}\left(x_{1}, \ldots, x_{\tilde{M}_{i}}\right) \geq \beta_{1}+\tau^{*} / 7
$$

for $i$ sufficiently large, in contradiction to the definition of $N\left(\alpha_{i}, \beta_{1}, \theta_{1}\right)$, which completes the proof of the theorem.

The question arises whether we can apply theorem 4.1 .1 to the LR test, for which we have proved several results on its shortcoming. However, in theorem 4.1.1 it is assumed that the shortcoming of the sequence of tests tends to zero for each sequence $\left\{\alpha_{n}\right\}$ with $\lim _{n \rightarrow \infty} \alpha_{n}=0$. In chapter II and III most of the theorems are valid only if $\alpha_{n}$ does not decrease too fast. Nevertheless theorem 4.1.1. can often be applied since this condition essentially serves to ensure uniform convergence to zero of the shortcoming. Since in chapter $\mathrm{V}$ stronger results about the Bahadur deficiency of the LR test will be proved, we do not mention here explicitely such corollaries to theorem 4.1.1 and the theorems of chapter II and III.

\subsection{EXAMPLES}

The first example shows that even for a sequence of tests $\left\{\phi_{n}\right\}$, which is deficient in the sense of Bahadur of order $O$ (1) uniformly in $\theta$ as $\alpha \rightarrow 0$ (the definition of this concept is similar to (1.1.4)) the shortcoming will not necessarily tend to zero uniformly on $\theta_{1}$ for all vanishing sequences $\left\{\alpha_{n}\right\}$.

In the second example it will be shown that uniform convergence of the shortcoming to zero is unable to strenghten the statements about Bahadur deficiency.

EXAMPLE 4.2.1. Let $\mathrm{x}_{1}, \mathrm{x}_{2}, \ldots$ be a sequence of $\mathrm{i} . \mathrm{i} . \mathrm{d}$. normal $\mathrm{N}(\theta, 1)$ random variables. Consider the testing problem $\mathrm{H}_{0}: \theta \leq 0$ against $\mathrm{H}_{1}: \theta>0$. The sequence of tests $\left\{\phi_{n}^{\gamma}\right\}$ has the following form:

$$
\phi_{n}^{\gamma}\left(x_{1}, \ldots, x_{n}\right)=\left\{\begin{array}{l}
1 \\
\text { if } \sum_{i=1}^{n-1} x_{i} \gamma \quad n=2,3, \ldots . \\
0
\end{array}\right.
$$

The sequence of tests $\left\{\phi_{n}^{\gamma}\right\}$ is obviously deficient in the sense of Bahadur of order $O(1)$ uniformly in $\theta$.

We investigate the shortcoming of this test in $\theta_{n}=n^{\frac{1}{2}}$ for levels of significance $\alpha_{n}=\Phi(-n), n=2,3, \ldots$, where $\Phi(x)=P_{0}\left(x_{1} \leq x\right)$. 
The envelope power function in $\theta_{n}$ equals $\frac{1}{2}$. $n(n-1)^{\frac{1}{2}}$. This implies

We determine $\gamma_{n}\left(\alpha_{n}\right) \cdot P_{0}\left(\sum_{i=1}^{n-1} x_{i} \geq \gamma_{n}\left(\alpha_{n}\right)\right)=\Phi(-n)$, hence $\gamma_{n}\left(\alpha_{n}\right)=$

$$
\lim _{n \rightarrow \infty} E_{\theta_{n}}{ }_{n}^{\gamma_{n}\left(\alpha_{n}\right)}\left(x_{1}, \ldots, x_{n}\right)=\Phi\left(-\frac{1}{2}\right),
$$

and thus the shortcoming does not tend uniformly to zero.

The second example concerns the following: let $\left\{n_{n}\right\}$ be a sequence of positive numbers with $\lim _{n \rightarrow \infty} \eta_{n}=0$; then there exists a testing problem and a sequence of tests $\left\{\phi_{n}^{\gamma}\right\}$ such that

(i) the shortcoming of $\left\{\phi_{n}^{\gamma}\right\}$ tends to zero uniformly over the whole set of alternatives for each sequence of levels $\left\{\alpha_{n}\right\}$ tending to zero,

(ii) for some $\beta \in(0,1)$ and $\theta \in$ int $\theta_{1}$ it holds that $N_{\phi}(\alpha, \beta, \theta)-N \geq \eta_{N} N^{\frac{1}{2}}$, where $\mathrm{N}=\mathrm{N}^{+}(\alpha, \beta, \theta)$, and

(iii) although $\left\{\phi_{n}^{\gamma}\right\}$ is deficient in the sense of Bahadur at $\theta$ of order $O\left(\mathrm{~N}^{+}(\alpha, \beta, \theta)^{\frac{n}{2}}\right)$ as $\alpha \rightarrow 0$, the convergence is not uniform in $\theta$.

Hence the $O\left(\mathrm{~N}^{+}(\alpha, \beta, \theta)^{\frac{1}{2}}\right)$ term in theorem 4.1 .1 can not be improved upon and uniformly vanishing shortcoming does not imply uniform convergence for the Bahadur deficiency.

EXAMPLE 4.2.2. Let $\mathrm{x}_{1}, \mathrm{x}_{2}, \ldots$ be i.i.d. normal $\mathrm{N}(\theta, 1)$ random variables. We consider the testing problem $\mathrm{H}_{0}: \theta \leq 0$ against $\mathrm{H}_{1}: \theta>0$.

Without loss of generality assume that $n_{n} n^{\frac{1}{2}} \geq 4$. Denote by $[a]$ the smallest integer $\geq a$, i.e. $[a]=-\operatorname{entier}(-a)$.

The sequence of tests $\left\{\phi_{n}^{\gamma}\right\}$ has the following form:

$$
\phi_{n}^{\gamma_{n}(\alpha)}\left(x_{1}, \ldots, x_{n}\right)=\left\{\begin{array}{c}
1 \\
1-\frac{1-\alpha}{\Phi\left(u_{\alpha}+n\right.}\left[u_{\alpha}^{2}\right)
\end{array} \text { if } n^{-\frac{1}{2}} \sum_{i=1}^{n} x_{i}<u_{\alpha}+n\left[u_{\alpha}^{2}\right]^{\prime}\right.
$$

where $u_{\alpha}$ is defined by $\Phi\left(u_{\alpha}\right)=1-\alpha$.

Let $\left\{\alpha_{n}\right\}$ be some sequence tending to zero; then $\eta_{\left[u_{\alpha_{n}}\right]} \rightarrow 0$ as $n \rightarrow \infty$ and hence

$$
\lim _{n \rightarrow \infty}\left\{P_{\theta_{n}}(n^{\frac{1}{2}} \bar{x}_{n} \geq u_{\alpha_{n}}+\underbrace{2}_{\left[u_{n}\right]})-P_{\theta_{n}}\left(n^{\frac{1}{2}} \bar{x}_{n} \geq u_{\alpha_{n}}\right)\right\}=0
$$

for each sequence $\left\{\theta_{n}\right\}$, implying that the shortcoming tends to zero uniformly 
over the whole set of alternatives.

It is easy to verify that

$$
\mathrm{N}^{+}\left(\alpha, \frac{1}{2}, \theta\right)=\left[\mathrm{u}_{\alpha}^{2} \theta^{-2}\right] .
$$

Let $\mathrm{m}=\operatorname{entier}\left(\left[u_{\alpha}^{2} \theta^{-2}\right]^{\frac{1}{2}}+\frac{1}{2} \theta^{-1} \eta_{\left[u_{\alpha}^{2}\right]}\right)^{2}$. Since

$$
u_{\alpha}-\theta\left[u_{\alpha}^{2} \theta^{-2}\right]^{\frac{1}{2}}+\left[u_{\alpha}^{2}\right]^{-\frac{1}{2}} \geq 0 \quad \text { for all } \theta \leq 1
$$

and, by assumption,

$$
{ }_{\left[u_{\alpha}^{2}\right]}^{\frac{1}{4} \pi} \geq\left[u_{\alpha}^{2}\right]^{-\frac{1}{2}}
$$

it follows that

$$
u_{\alpha}-\theta m^{\frac{1}{2}}+\eta_{\left[u_{\alpha}^{2}\right]} \geq{ }_{\left[u_{\alpha}^{2}\right]}^{\frac{1}{4} \eta} \quad(\theta \leq 1) .
$$

On the other hand

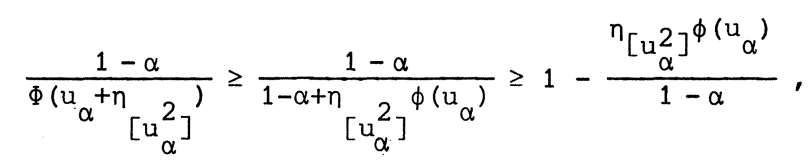

where $\phi$ denotes the derivative of $\Phi$. Therefore

$$
\begin{aligned}
& \mathrm{E}_{\theta} \phi_{\mathrm{m}}^{\gamma_{\mathrm{m}}(\alpha)}\left(\mathrm{x}_{1}, \ldots, \mathrm{x}_{\mathrm{m}}\right) \leq \\
& \leq P_{\theta}\left(\bar{x}_{m} m^{\frac{1}{2}}-\theta m^{\frac{1}{2}} \geq u_{\alpha}-\theta m^{\frac{1}{2}}+\eta u_{\alpha}^{2}\right)^{\prime}+1-\frac{1-\alpha}{\Phi\left(u_{\alpha}+\eta u_{\alpha}^{2}{ }^{\prime}\right.} \\
& \leq P_{\theta}\left(\bar{x}_{m} m^{\frac{1}{2}}-\theta m^{\frac{1}{2}} \geq \frac{1 \frac{1}{4} \eta}{\left[u_{\alpha}^{2}\right]}\right)+\frac{\left.\eta_{\left[u_{\alpha}^{2}\right]}\right]^{\phi\left(u_{\alpha}\right)}}{1-\alpha}<\frac{1}{2}
\end{aligned}
$$

for $\alpha$ sufficiently small and $\theta \leq 1$. Hence

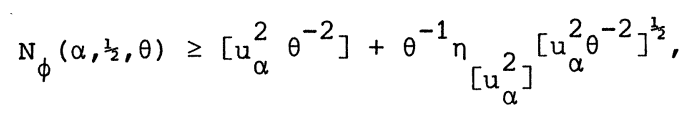


implying

$$
\left.\left\{\mathrm{N}_{\phi}\left(\alpha, \frac{1}{2}, \theta\right)-\mathrm{N}^{+}\left(\alpha, \frac{1}{2}, \theta\right)\right\}\left\{\mathrm{N}^{+}\left(\alpha, \frac{1}{2}, \theta\right)\right\}^{-\frac{1}{2}} \geq \theta^{-1}{ }^{-1} \mathrm{u}_{\alpha}^{2}\right]
$$

for all $\theta \leq 1$ and $\alpha$ sufficiently small.

Choosing $\theta=1$ and $\theta=n_{\left[u_{\alpha}^{2}\right]}$ respectively the properties (ii) and (iii) are established. 


\section{BAHADUR DEFICIENCY OF THE}

\section{LIKELIHOOD RATIO TEST}

\subsection{INTRODUCTION}

In chapter II and III we have chosen as a measure of optimality the maximum shortcoming of a test. In this chapter we shall consider Bahadur deficiency as a yardstick to measure the performance of LR tests.

As we have already mentioned in chapter I the LR test is, under some conditions, efficient in the Bahadur sense. However, Bahadur efficiency is not a very sharp instrument for studying optimality of tests; a more informant measure is provided by the concept of Bahadur deficiency.

We first introduce some notation. Let $\left\{\phi_{n}^{\gamma} ; \gamma \in \Gamma\right\}$ be a family of tests, where $\Gamma$ is an index set with the following interpretation: let $\theta_{0}$ be some subset of $\theta$ (the null hypothesis) and let $0<\alpha<1$ (the level of significance); then there exists one and only one $\gamma \in \Gamma$, denoted by $\gamma_{n}(\alpha)$, such that $\sup _{\theta_{0} \in \theta_{0}} E_{\theta_{0}} \phi_{n}^{\gamma_{n}(\alpha)}\left(x_{1}, \ldots, x_{n}\right)=\alpha$. For the families of tests considered in this chapter it holds that

$$
\text { (5.1.1) } \quad \alpha>\alpha^{\prime} \text { implies } E_{\theta} \phi_{n}^{\gamma_{n}(\alpha)}\left(x_{1}, \ldots, x_{n}\right) \geq E_{\theta} \phi_{n}^{\gamma_{n}\left(\alpha^{\prime}\right)}\left(x_{1}, \ldots, x_{n}\right)
$$

for all $\theta \in \theta-\theta_{0}$.

Define for $0<\beta<1$ and $\theta \in \theta-\theta_{0}$

(5.1.2) $\quad \alpha_{n}(\beta, \theta)=\min \left\{\alpha ; E_{\theta} \phi_{n}^{\gamma_{n}(\alpha)}\left(x_{1}, \ldots, x_{n}\right) \geq \beta\right\}, \quad n=1,2, \ldots$.

Hence for all $0<\alpha<1,0<\beta<1$ and $\theta \in \theta-\Theta_{0}$

(5.1.3) $\quad \alpha_{N(\alpha, \beta, \theta)}(\beta, \theta) \leq \alpha<\alpha_{N(\alpha, \beta, \theta)-1}(\beta, \theta)$,

where $N(\alpha, \beta, \theta)$ is defined by 


$$
N(\alpha, \beta, \theta)=\min \left\{n_{i} E_{\theta} \phi_{m}^{\gamma_{m}(\alpha)}\left(x_{1}, \ldots, x_{m}\right) \geq \beta, m \geq n\right\},
$$

for $0<\beta<1$ and $\theta \in \theta-\theta_{0}$.

Let us now consider some examples.

EXAMPLE 5.1.1. Consider a sequence of i.i.d. random variables with a normal $N(\theta, 1)$ distribution and suppose $H_{0}: \theta=0$ is to be tested against $H_{1}: \theta \neq 0$. The LR test is two-sided, the MP test for $\mathrm{H}_{0}$ against $\mathrm{H}_{1}^{*}: \theta=\theta^{*}$ is one-sided. It turns out that in this case the LR test is deficient in the sense of Bahadur at $\theta$ of order $O(1)$ as $\alpha \rightarrow 0$ for all $\theta \neq 0$. The definition of this concept is similar to $(1.1 .4)$. Let $\theta^{*}>0$ be fixed; simple calculations lead to

$$
N^{L R}\left(\alpha, \beta, \theta^{*}\right) \leq\left(u_{\frac{1}{2} \alpha}-u_{\beta}\right)^{2}\left(\theta^{*}\right)^{-2}+1,
$$

where $u_{\alpha}$ is defined by

$$
P_{0}\left(\mathrm{x} \leq \mathrm{u}_{\alpha}\right)=\Phi\left(\mathrm{u}_{\alpha}\right)=1-\alpha \quad(0<\alpha<1),
$$

and

$$
\mathbf{N}^{+}\left(\alpha, \beta, \theta^{*}\right) \geq\left(u_{\alpha}-u_{\beta}\right)^{2}\left(\theta^{*}\right)^{-2}
$$

Since $\lim _{\alpha \rightarrow 0}\left\{\left(u_{z_{2} \alpha}-u_{\beta}\right)^{2}\left(\theta^{*}\right)^{-2}+1-\left(u_{\alpha}-u_{\beta}\right)^{2}\left(\theta^{*}\right)^{-2}\right\}=2\left(\theta^{*}\right)^{-2} \log 2+1$, the LR test is deficient in the sense of Bahadur at $\theta^{*}$ of order $O(1)$ as $\alpha \rightarrow 0$.

EXAMPLE 5.1.2. In this example we consider the simplest non-trivial case of a discrete distribution: $\mathrm{x}_{1}, \mathrm{x}_{2}, \ldots$ are independent Bernoulli random variables with $\operatorname{Pr}\left(x_{i}=1\right)=p \in(0,1)$. Putting $\mu(0)=\mu(1)=\frac{1}{2}$ and $\theta=\log \left(\frac{p}{1-p}\right)$ we obtain an exponential family model.

We test the null hypothesis

$$
\mathrm{H}_{0}: \log \left(2 \cdot 3^{-\frac{1}{2}}-1\right) \leq \theta \leq-\log 3 .
$$

Note that $\lim _{\theta \rightarrow-\infty} I\left(\theta, \log \left(2.3^{-\frac{1}{2}}-1\right)\right)=I(0,-\log 3)$. To obtain a power $\frac{1}{2}$ at $\theta=0$ the critical region of the LR test has to be of the form $\bar{x}_{n}>\frac{1}{2}$ with randomization in $\bar{x}_{n}=0$ and $\bar{x}_{n}=\frac{1}{2}$, if $n$ is even. Hence the LR test has the following form

$$
\phi_{n}^{L R}(x)= \begin{cases}1 & > \\ \delta_{n} \text { if } \sup _{\theta_{0} \epsilon \Theta_{0}}\left\{\theta_{0}^{\prime} x-\psi\left(\theta_{0}\right)\right\}-\sup _{\theta \in \theta^{\prime}}\left\{\theta^{\prime} x-\psi(\theta)\right\} & =I(0,-\log 3), \\ 0 & <\end{cases}
$$

with 


$$
\delta_{n}=\left\{\begin{array}{cc}
0 & n \text { odd } \\
\left.\frac{\frac{1}{2}\left(\frac{1}{2} n\right.}{n}\right) & n \text { even }
\end{array}\right.
$$

Since by formula (12) in HOEFFDING (1965b)

and

$$
P-\log 3\left(\bar{x}_{n} \geq \frac{1}{2}\right)=\exp \left\{-\mathrm{nI}(0,-\log 3)-\frac{1}{2} \log \mathrm{n}+O(1)\right\}
$$

$$
P \log \left(2.3^{-\frac{1}{2}}-1\right)\left(\bar{x}_{n}=0\right)=\exp \{-n I(0,-\log 3)\},
$$

$$
\text { (5.1.4) } \quad \alpha_{n}^{L R}\left(\frac{1}{2}, 0\right)= \begin{cases}\frac{1}{2} \exp \{-\mathrm{nI}(0,-\log 3)+o(1)\} & \mathrm{n} \text { even } \\ \exp \left\{-\mathrm{nI}(0,-\log 3)-\frac{1}{2} \log \mathrm{n}+O(1)\right\} & \mathrm{n} \text { odd }\end{cases}
$$

(cf. (5.1.2)). It follows that

(5.1.5) $\quad \mathrm{N}^{\mathrm{LR}}\left(\alpha, \frac{1}{2}, 0\right)=\frac{-\log \alpha}{I(0,-\log 3)}+O(1)$

(cf. $(5.1 .3))$.

On the other hand the MP test of $\mathrm{H}_{0}$ against $\mathrm{H}_{1}^{*}: \theta=0$ with power $\frac{1}{2}$ at $\theta=0$ is given by

$$
\phi_{n}^{+}(x)=\left\{\begin{array}{lll}
1 & > \\
\frac{1}{2} & \text { if } & x=\frac{1}{2} \\
0 & <
\end{array}\right.
$$

and thus $\alpha_{n}^{+}\left(\frac{1}{2}, 0\right)=\exp \left\{-n I(0,-\log 3)-\frac{1}{2} \log n+O(1)\right\}$. Hence

$$
\text { (5.1.6) } \quad \mathrm{N}^{+}\left(\alpha, \frac{1}{2}, 0\right)=\frac{-\log \alpha}{I(0,-\log 3)}-\frac{\frac{1}{2}}{I(0,-\log 3)} \log \left(\frac{-\log \alpha}{I(0,-\log 3)}\right)+O(1) \text {. }
$$

In combination with (5.1.5) it follows that

$$
\lim _{\alpha \rightarrow 0} \frac{N^{L R}\left(\alpha, \frac{1}{2}, 0\right)-N^{+}\left(\alpha, \frac{1}{2}, 0\right)}{\log N^{+}\left(\alpha, \frac{1}{2}, 0\right)}=\frac{\frac{1}{2}}{I(0,-\log 3)} .
$$

Thus the LR test is not deficient in the sense of Bahadur at 0 of order $O(1)$, but it can be shown that its deficiency is of order $O\left(\log \mathrm{N}^{+}(\alpha, \beta, 0)\right)$.

In the first example the LR test is deficient in the sense of Bahadur of order $O(1)$ and in the second exemple its deficiency at $\theta=0$ is of order 
$O\left(\log \mathrm{N}^{+}(\alpha, \beta, 0)\right)$. The difference is explained by the fact that in the second example the critical region of the LR test has points in common with the boundary of the sample space. It turns out that these examples are representative for testing problems in one-dimensional exponential families. The theory will be developed in section 2 .

In the k-dimensional case the situation is more complicated. Here usually the LR test is deficient in the sense of Bahadur at $\theta$ of order $O\left(\log \mathrm{N}^{+}(\alpha, \beta, \theta)\right)$ as $\alpha \rightarrow 0$. This will be proved in section 3 .

\subsection{THE ONE-DIMENSIONAL CASE}

In this section it will be assumed that the random variables are distributed according to a one-parameter exponential family. We consider the testing problem $\mathrm{H}_{0}: \theta \in \theta_{0}$ against $\mathrm{H}_{1}: \theta \in \theta_{1}=\theta-\theta_{0}$.

The first theorem concerns the case that $\theta_{0}$ is contained in a compact subset of int $\theta$.

THEOREM 5.2.1. Let $\theta_{0} \subset \mathrm{K} \subset$ int $\theta$, where $\mathrm{K}$ is a compact set. Let $\mathrm{I}\left(\theta_{0}\right)$ be defined as in (3.2.1). If $\theta \in$ int $\theta_{1}$ satisfies $I\left(\theta, \theta_{0}\right)<I\left(\theta_{0}\right)$ then the LR test is deficient in the sense of Bahadur at $\theta_{1}$ of order $O(1)$ as $\alpha \rightarrow 0$.

PROOF. The proof is based on an expansion of $\log \alpha_{n}^{+}(\beta, \theta)$ and a similar one of $\log \alpha_{n}^{L R}(\beta, \theta)$. Combination of these expansions easily yields the theorem. Let $\theta_{1} \in$ int $\theta_{1}$. Then we shall prove that for all $0<\beta<1$, as $n \rightarrow \infty$,

$$
\log \alpha_{n}^{+}\left(\beta, \theta_{1}\right)=-n I\left(\theta_{1}, \theta_{0}\right)+n^{\frac{1}{2}} B\left(\beta, \theta_{1}\right)-\frac{1}{2} \log n+O(1)
$$

where

$$
B\left(\beta, \theta_{1}\right)=\left|\theta_{0}^{*}-\theta_{1}\right| \sigma\left(\theta_{1}\right) \Phi^{-1}(\beta)
$$

if $\theta_{0}^{*} \in \operatorname{cl} \theta_{0}$ is uniquely defined by $I\left(\theta_{1}, \theta_{0}^{*}\right)=I\left(\theta_{1}, \theta_{0}\right)$, and

$$
B\left(\beta, \theta_{1}\right)=\left(\tilde{\theta}_{0}-\theta_{1}\right) \sigma\left(\theta_{1}\right) \gamma
$$

if there are two points $\theta_{0}^{*}, \tilde{\theta}_{0} \in \operatorname{cl} \theta_{0}$ such that $\theta_{0}^{*}<\theta_{1}<\tilde{\theta}_{0}$ and $I\left(\theta_{1}, \theta_{0}^{*}\right)=$ $I\left(\theta_{1}, \tilde{\theta}_{0}\right)=I\left(\theta_{1}, \theta_{0}\right)$. Here $\gamma>0$ is defined by $\Phi(\gamma)-\Phi\left(\left(\theta_{1}-\tilde{\theta}_{0}\right)\left(\theta_{1}-\theta_{0}^{*}\right)^{-1} \gamma\right)=\beta$.

We distinghuish several cases. 
(a) Assume that inf $\theta_{0}<\theta_{1}<\sup \theta_{0}$. Let $\theta_{0}^{*}=\sup \left\{\theta_{0} \epsilon \theta_{0} ; \theta_{0}<\theta_{1}\right\}$ and $\tilde{\theta}_{0}=$ $\inf \left\{\theta_{0} \epsilon \theta_{0} ; \theta_{0}>\theta_{1}\right\}$. The MP size- $\alpha$ test for $\mathrm{H}_{0}$ against $\mathrm{H}_{1}^{*}: \theta=\theta_{1}$ has the following form

$$
\phi_{n}^{+}(x)=\left\{\begin{array}{ccc}
1 & \epsilon\left(c_{n}, c_{n}^{*}\right) \\
\gamma_{n} & \text { if } & x=c_{n} \text { or } c_{n}^{*} \\
0 & & \notin\left(c_{n}, c_{n}^{*}\right)
\end{array}\right.
$$

with $\gamma_{n}, c_{n}$ and $c_{n}^{*}$ such that

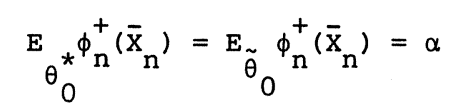

(cf. LEHMANN (1959) section 3.7). For $\alpha=\alpha_{n}^{+}\left(\beta, \theta_{1}\right)$ the constants $\gamma_{n}, c_{n}$ and $c_{n}^{*}$ are such that $E_{\theta_{1}} \phi_{n}^{+}\left(\bar{x}_{n}\right)=\beta$.

We have two subcases

(i) $I\left(\theta_{1}, \theta_{0}^{*}\right) \neq I\left(\theta_{1}, \tilde{\theta}_{0}\right)$

(ii) $I\left(\theta_{1}, \theta_{0}^{*}\right)=I\left(\theta_{1}, \tilde{\theta}_{0}\right)$.

We first consider (i) and assume that $I\left(\theta_{1}, \theta_{0}^{*}\right)<I\left(\theta_{1}, \tilde{\theta}_{0}\right)$ (the reverse inequality can be treated similarly); then $I\left(\theta_{1}, \theta_{0}\right)=I\left(\theta_{1}, \theta_{0}^{*}\right)$.

Since $\theta_{1}$ lies closer to $\theta_{0}^{*}$ than to $\tilde{\theta}_{0}$, in $I^{\prime}$ distance, it will turn out that $c_{n}^{*}$ does not play an important part in the determination of $\alpha_{n}^{+}\left(\beta, \theta_{1}\right)$. Define $\bar{\theta}$ as a sort of centre of the interval $\left(\theta_{0}^{*}, \tilde{\theta}_{0}\right)$ measured in I'distance: $I\left(\bar{\theta}, \theta_{0}^{*}\right)=I\left(\bar{\theta}_{,} \tilde{\theta}_{0}\right)$. We now have

(5.2.4) $\underset{n \rightarrow \infty}{\lim \inf } c_{n}^{*} \geq \lambda(\bar{\theta})$.

For, suppose $\lim _{n \rightarrow \infty} \inf _{n} c_{n}^{*}<\lambda(\bar{\theta})$, then there exists a subsequence $\left\{n_{i}\right\}$ with $\mathrm{c}_{\mathrm{n}_{i}}^{\star}<\lambda(\bar{\theta}) ;$ hence

$$
\begin{aligned}
\alpha_{n_{i}}^{+}\left(\beta, \theta_{1}\right) & \leq P_{\tilde{\theta}_{0}}\left(\bar{x}_{n_{i}} \leq c_{n_{i}^{*}}^{*}\right) \leq \\
& \leq P_{\tilde{\theta}_{0}}\left(\bar{x}_{n_{i}} \leq \lambda(\bar{\theta})\right) \\
& =\int_{x \leq \lambda(\bar{\theta})} \exp \left\{n_{i}\left(\tilde{\theta}_{0}-\bar{\theta}\right) x-n_{i} \psi\left(\tilde{\theta}_{0}\right)+n_{i} \psi(\bar{\theta})\right\} d \bar{P}_{\bar{\theta}}^{-n}(x) \\
& \leq \exp \left\{-n_{i} I\left(\bar{\theta}_{,} \tilde{\theta}_{0}\right)\right\} .
\end{aligned}
$$

On the other hand for sufficiently large a $\in \mathbb{R}$ 


$$
P_{\theta_{1}}\left(\overline{\mathrm{x}}_{\mathrm{n}}>\lambda\left(\theta_{1}\right)+\operatorname{an}^{-\frac{1}{2}}\right)<\frac{1}{2} \beta,
$$

and hence

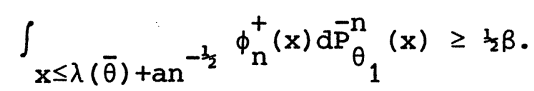

This implies that

$$
\begin{aligned}
\alpha_{n}^{+}\left(\beta, \theta_{1}\right) \geq & \int \phi_{n}^{+}(x) \exp \left\{n\left(\theta_{0}^{*}-\theta_{1}\right) x-n \psi\left(\theta_{0}^{*}\right)+n \psi\left(\theta_{1}\right)\right\} d P_{\theta_{1}}^{-n}(x) \\
& x \leq \lambda\left(\theta_{1}\right)+a^{-\frac{1}{2}} \\
\geq & \frac{1}{2} \beta \exp \left\{-n I\left(\theta_{1}, \theta_{0}^{*}\right)+n^{\frac{1}{2}}\left(\theta_{0}^{*}-\theta_{1}\right) a\right\} .
\end{aligned}
$$

In combination with $(5.2 .5)$ and $I\left(\theta_{1}, \theta_{0}^{*}\right)<I\left(\bar{\theta}_{,} \theta_{0}^{*}\right)$ we obtain a contradiction for large $n$, completing the proof of (5.2.4).

Consequently

(5.2.7) $\quad P_{\theta_{1}}\left(\bar{x}_{n} \geq c_{n}^{*}\right) \leq \exp \left\{-n I\left(\theta_{1}, \lambda^{-1}\left(c_{n}^{*}\right)\right)\right\} \leq$

$$
\leq \exp \left\{-\frac{1}{2} n I\left(\theta_{1}, \bar{\theta}\right)\right\}
$$

for large $n$. The points $c_{n}$ and $c_{n}^{*}$ have to satisfy

$$
P_{\theta_{1}}\left(\bar{x}_{\mathrm{n}} \in\left(\mathrm{c}_{\mathrm{n}}, \mathrm{c}_{\mathrm{n}}^{*}\right)\right) \leq \beta \leq P_{\theta_{1}}\left(\overline{\mathrm{x}}_{\mathrm{n}} \in\left[\mathrm{c}_{\mathrm{n}}, \mathrm{c}_{\mathrm{n}}^{*}\right]\right) .
$$

Furthermore (5.2.7) and the Berry-Esseen theorem imply that $P_{\theta_{1}}\left(\bar{x}_{n} \in\left(c_{n}, c_{n}^{*}\right)\right)$ as well as $P_{\theta_{1}}\left(\bar{x}_{n} \in\left[c_{n}, c_{n}^{*}\right]\right)$ are equal to $1-\Phi\left(\left\{c_{n}-\lambda\left(\theta_{1}\right)\right\} \sigma\left(\theta_{1}\right)^{-1} n^{\frac{1}{2}}\right)+O\left(n^{-\frac{1}{2}}\right)$. Hence $\Phi\left(\left\{c_{n}-\lambda\left(\theta_{1}\right)\right\}_{\sigma}\left(\theta_{1}\right)^{-1} n^{\frac{1}{2}}\right)=1-\beta+O\left(n^{-\frac{1}{2}}\right)$, and therefore

$$
c_{n}=\lambda\left(\theta_{1}\right)-\sigma\left(\theta_{1}\right) \Phi^{-1}(\beta) n^{-\frac{1}{2}}+O\left(n^{-1}\right) .
$$

Next we express $\alpha_{n}^{+}\left(\beta, \theta_{1}\right)$ in terms of $c_{n}$ :

$$
\begin{aligned}
& \alpha_{n}^{+}\left(\beta, \theta_{1}\right) \geq P_{\theta_{0}^{*}}\left(\bar{x}_{n} \in\left(c_{n}, c_{n}^{*}\right)\right) \geq \\
& \geq \exp \left\{-n I\left(\lambda^{-1}\left(c_{n}\right), \theta_{0}^{*}\right)\right\} \int \exp \left\{n^{\frac{1}{2}}\left(\theta_{0}^{*}-\lambda^{-1}\left(c_{n}\right)\right) \sigma\left(\lambda^{-1}\left(c_{n}\right)\right) y\right\} d \tilde{P}^{n} \lambda^{-1}\left(c_{n}\right)(y), \\
& \left(0,\left\{c_{n}^{*}-c_{n}\right\} \sigma\left(\lambda^{-1}\left(c_{n}\right)\right)^{-1} n^{\frac{1}{2}}\right)
\end{aligned}
$$

where $\tilde{P}_{\theta}^{n}$ denotes the distribution of $\left\{\bar{x}_{n}-\lambda(\theta)\right\} \sigma(\theta)^{-1} n^{\frac{1}{2}}$ under $\theta$. Using the 
Berry-Esseen theorem we see that

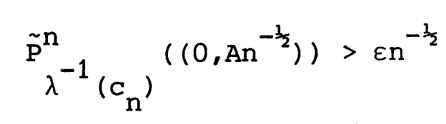

for sufficiently large $A$ and some $\varepsilon>0$ provided $n$ is so large that $c_{n}$ is close to $\lambda\left(\theta_{1}\right)$. Since furthermore $\lim _{n \rightarrow \infty}\left(c_{n}^{*}-c_{n}\right) \sigma\left(\lambda^{-1}\left(c_{n}\right)\right)^{-1} n^{\frac{1}{2}}=\infty$ by (5.2.4) and $(5.2 .8)$ there exists a constant $\delta>0$ such that

(5.2.9) $\quad \alpha_{n}^{+}\left(\beta, \theta_{1}\right) \geq \delta n^{-\frac{1}{2}} \exp \left\{-n I\left(\lambda^{-1}\left(c_{n}\right), \theta_{0}^{*}\right)\right\}$

On the other hand

$$
\begin{aligned}
\alpha_{n}^{+}\left(\beta, \theta_{1}\right) & \leq P_{\theta_{0}^{*}}\left(\bar{x}_{n} \in\left[c_{n^{\prime}}, \infty\right)\right)= \\
& =\exp \left\{-n I\left(\lambda^{-1}\left(c_{n}\right), \theta_{0}^{*}\right)\right\} \sum_{j=0}^{\infty} \int_{\left[j n^{-\frac{1}{2}},(j+1) n^{-\frac{1}{2}}\right)}^{\left.\exp n^{\frac{1}{2}}\left(\theta^{*}-\lambda^{-1}(c)\right) \sigma\left(\lambda^{-1}\left(c_{n}\right)\right) y\right\} d P^{n} \lambda^{-1}\left(c_{n}\right)}(y) .
\end{aligned}
$$

The Berry-Esseen theorem implies the existence of a constant $c$ * such that $\tilde{P}_{\lambda^{n}}^{n}\left(c_{n}\right)\left(\left[j n^{-\frac{1}{2}},(j+1) n^{-\frac{1}{2}}\right)\right) \leq c^{*} n^{-\frac{1}{2}}$ for all $j$, and hence for large $n$

$$
\begin{aligned}
\alpha_{n}^{+}\left(\beta, \theta_{1}\right) & \leq c^{*} n^{-\frac{1}{2}} \exp \left\{-n I\left(\lambda^{-1}\left(c_{n}\right), \theta_{0}^{*}\right)\right\} \sum_{j=0}^{\infty} \exp \left\{\left(\theta_{0}^{*}-\lambda^{-1}\left(c_{n}\right)\right) \sigma\left(\lambda^{-1}\left(c_{n}\right)\right) j\right\} \\
& \leq \tilde{c}^{-\frac{1}{2}} \exp \left\{-n I\left(\lambda^{-1}\left(c_{n}\right), \theta_{0}^{*}\right)\right\}
\end{aligned}
$$

for some constant $0<\tilde{c}<\infty$.

Combining $(5.2 .9)$ and $(5.2 .10)$ we find

$$
\log \alpha_{n}^{+}\left(\beta, \theta_{1}\right)=-n I\left(\lambda^{-1}\left(c_{n}\right), \theta_{0}^{*}\right)-\frac{1}{2} \log n+O(1) .
$$

It remains to expand $I\left(\lambda^{-1}\left(c_{n}\right), \theta_{0}^{*}\right)$ in powers of $n$. Since

$$
(5.2 .11) \quad I\left(\vartheta, \theta_{0}^{*}\right)=I\left(\theta_{1}, \theta_{0}^{*}\right)+\left(\vartheta-\theta_{1}\right)\left(\theta_{1}-\theta_{0}^{*}\right) \sigma^{2}\left(\theta_{1}\right)+O\left(\left(\vartheta-\theta_{1}\right)^{2}\right)
$$

for $\vartheta \rightarrow \theta_{1}$, and

(5.2.12) $\quad \lambda^{-1}(x)=\lambda^{-1}(y)+(x-y) \sigma^{-2}\left(\lambda^{-1}(y)\right)+O\left((x-y)^{2}\right)$

for $x \rightarrow y$, and thus 
(5.2.13) $I\left(\lambda^{-1}(x), \theta_{0}^{*}\right)=I\left(\lambda^{-1}(y), \theta_{0}^{*}\right)+(x-y)\left(\theta_{1}-\theta_{0}^{*}\right)+O\left((x-y)^{2}\right)$

for $x \rightarrow y,(5.2 .8)$ implies

$$
I\left(\lambda^{-1}\left(c_{n}\right), \theta_{0}^{*}\right)=I\left(\theta_{1}, \theta_{0}^{*}\right)-\sigma\left(\theta_{1}\right) \Phi^{-1}(\beta)\left(\theta_{1}-\theta_{0}^{*}\right) n^{-\frac{1}{2}}+O\left(n^{-1}\right)
$$

as $n \rightarrow \infty$. Therefore

$$
\log \alpha_{n}^{+}\left(\beta, \theta_{1}\right)=-n I\left(\theta_{1}, \theta_{0}^{*}\right)+n^{\frac{1}{2}} \sigma\left(\theta_{1}\right) \Phi^{-1}(\beta)\left(\theta_{1}-\theta_{0}^{*}\right)-\frac{1}{2} \log n+O(1) .
$$

So in subcase (i) we have proved (5.2.1).

In subcase (ii) we consider a point $\theta_{1}$ in the middle of the interval $\left(\theta_{0}^{*}, \tilde{\theta}_{0}\right)$ in the following sense: $I\left(\theta_{1}, \theta_{0}^{*}\right)=I\left(\theta_{1}, \tilde{\theta}_{0}\right)$. Both points $c_{n}$ and $c_{n}^{*}$ now play a role and we first prove that they both are near $\lambda\left(\theta_{1}\right)$. Suppose $\lim \inf _{n \rightarrow \infty} c_{n}<\lambda\left(\theta_{1}\right)$; then there exists a sequence $\left\{n_{i}\right\}$ with $\lim _{i \rightarrow \infty} c_{n_{i}}<$ $\lambda\left(\theta_{1}\right)$. Since $\lim$ inf ${ }_{i \rightarrow \infty} c_{n_{i}}^{*}<\lambda\left(\theta_{1}\right)$ would imply $\lim$ inf ${ }_{i \rightarrow \infty} P_{\theta_{1}}\left(\bar{x}_{n_{i}} \in\left[c_{n_{i}}, c_{n_{i}}^{*}\right]\right)$ $=0$, in contradiction to $P_{\theta_{1}}\left(\bar{x}_{n} \epsilon\left[c_{n}, c_{n}^{*}\right]\right) \geq \beta>0$ for all $n$, we obtain $\lim$ inf $_{i \rightarrow \infty} c_{n_{i}}^{*} \geq \lambda\left(\theta_{1}\right)$. On the other hand $\lim \sup _{i \rightarrow \infty} c_{n_{i}}^{*}>\lambda\left(\theta_{1}\right)$ would imply $\lim \sup _{i \rightarrow \infty} P_{\theta_{1}}\left(\bar{x}_{n} \in\left(c_{n_{i}}, c_{n_{i}}^{*}\right)\right)=1$, in contradiction to $P_{\theta_{1}}\left(\bar{x}_{n} \in\left(c_{n}, c_{n}^{*}\right)\right)$ $\leq \beta<1$ for all $n$, we obtain lim $\sup _{i \rightarrow \infty} c_{n_{i}}^{*} \leq \lambda\left(\theta_{1}\right)$, and hence $\lim _{i \rightarrow \infty} c_{n_{i}}^{*}=\lambda\left(\theta_{1}\right)$. Therefore there exists a point $\xi \in\left(\theta_{0}^{*}, \theta_{1}\right)$ satisfying

$$
\mathrm{c}_{\mathrm{n}_{i}}<\lambda(\xi)<\lambda(\xi)+n_{1}<\mathrm{c}_{\mathrm{n}_{i}}^{*}
$$

for all $i$ and some $n_{1}>0$. It follows that

$$
\begin{aligned}
& \alpha_{n_{i}^{+}}^{+}\left(\beta, \theta_{1}\right) \geq P_{\theta_{0}^{*}}\left(\bar{x}_{n_{i}} \in\left(\lambda(\xi), \lambda(\xi)+n_{1}\right)\right) \geq \\
& \begin{aligned}
\geq \exp \left\{-n_{i} I\left(\xi, \theta_{0}^{*}\right)\right\} & \int \exp \left\{n_{i}\left(\theta_{0}^{*}-\xi\right)(x-\lambda(\xi))\right\} d \bar{p}_{j}^{n_{i}}(x) \\
& \left(\lambda(\xi), \lambda(\xi)+n_{1}\right)
\end{aligned} \\
& \geq n_{2} \exp \left\{-n_{i} I\left(\xi, \theta_{0}^{*}\right)-n_{i} n\left(\xi-\theta_{0}^{*}\right)\right\}
\end{aligned}
$$

for some $n_{2}>0$ and all $0<n<n_{1}$. Conversely

$$
\alpha_{n_{i}}^{+}\left(\beta, \theta_{1}\right) \leq P_{\tilde{\theta}_{0}}\left(\bar{x}_{n_{i}} \leq c_{n_{i}^{*}}^{*}\right) \leq \exp \left\{-n_{i} I\left(\lambda^{-1}\left(c_{n_{i}^{*}}^{*}\right), \tilde{\theta}_{0}\right)\right\}
$$

Since $I\left(\xi, \theta_{0}^{*}\right)<I\left(\lambda^{-1}\left(c_{n_{i}}^{*}\right), \tilde{\theta}_{0}\right)-\eta_{3}$ for some $\eta_{3}>0$ and all $i$, we obtain 
a contradiction by choosing $n$ sufficiently small. Thus $\lim$ inf ${ }_{n \rightarrow \infty} c_{n} \geq \lambda\left(\theta_{1}\right)$. Similarly it can be shown that $\lim \sup _{n \rightarrow \infty} c_{n}^{*} \leq \lambda\left(\theta_{1}\right)$. However, we also need some information about the rate of convergence of $c_{n}$ and $c_{n}^{*}$ to $\lambda\left(\theta_{1}\right)$. We therefore express $\alpha_{n}^{+}\left(\beta, \theta_{1}\right)$ in terms of $c_{n}$ and $c_{n}^{*}$ :

$$
\begin{aligned}
& \alpha_{n}^{+}\left(\beta, \theta_{1}\right)=\exp \left\{-n I\left(\lambda^{-1}\left(c_{n}\right), \theta_{0}^{*}\right)\right\} \times \\
& \times \int \tilde{\phi}_{n}^{+}(y) \exp \left\{n^{\frac{1}{2}}\left(\theta_{0}^{*}-\lambda^{-1}\left(c_{n}\right)\right) \sigma\left(\lambda^{-1}\left(c_{n}\right)\right) y\right\} \alpha \tilde{P}^{n} \lambda^{-1}\left(c_{n}\right) \\
& \quad\left[0,\left(c_{n}^{*}-c_{n}\right) \sigma\left(\lambda^{-1}\left(c_{n}\right)\right)^{-1} n^{\frac{1}{2}}\right]
\end{aligned}
$$

and

$$
\begin{aligned}
& \alpha_{n}^{+}\left(\beta, \theta_{1}\right)=\exp \left\{-n I\left(\lambda^{-1}\left(c_{n}^{*}\right), \tilde{\theta}_{0}\right)\right\} \times \\
& \times \int \tilde{\phi}_{n}^{+}(y) \exp \left\{n^{\frac{1}{2}}\left(\tilde{\theta}_{0}-\lambda^{-1}\left(c_{n}^{*}\right)\right) \sigma\left(\lambda^{-1}\left(c_{n}^{*}\right)\right) y\right\} d \tilde{P}^{n} \lambda^{-1}\left(c_{n}^{*}\right)(y), \\
& {\left[\left(c_{n}-c_{n}^{*}\right) \sigma\left(\lambda^{-1}\left(c_{n}^{*}\right)\right)^{-1} n^{\frac{1}{2}}, 0\right]}
\end{aligned}
$$

where $\tilde{\phi}_{n}^{+}(y)=\phi_{n}^{+}\left(y \sigma\left(\lambda^{-1}\left(c_{n}\right)\right) n^{-\frac{1}{2}}+c_{n}\right)$ in the first integral and $\tilde{\phi}_{n}^{+}(y)=$ $\phi_{n}^{+}\left(y \sigma\left(\lambda^{-1}\left(c_{n}^{*}\right)\right) n^{-\frac{1}{2}}+c_{n}^{*}\right)$ in the second integral. Similar to the proof in case (i) it can be shown that both integrals are of the form $n^{-\frac{1}{2}} e^{O(1)}$ as $n \rightarrow \infty$. This implies that

$$
I\left(\lambda^{-1}\left(c_{n}\right), \theta_{0}^{*}\right)=I\left(\lambda^{-1}\left(c_{n}^{*}\right), \tilde{\theta}_{0}\right)+O\left(n^{-1}\right) .
$$

Expanding both $I\left(\cdot, \theta_{0}^{*}\right)$ and $I\left(\cdot, \tilde{\theta}_{0}\right)$ we obtain

$$
\begin{aligned}
& \left(\lambda^{-1}\left(c_{n}\right)-\theta_{1}\right)\left(\theta_{1}-\theta_{0}^{*}\right) \sigma^{2}\left(\theta_{1}\right)+o\left(\left(\theta_{1}-\lambda^{-1}\left(c_{n}\right)\right)^{2}\right)= \\
& =\left(\lambda^{-1}\left(c_{n}^{*}\right)-\theta_{1}\right)\left(\theta_{1}-\tilde{\theta}_{0}\right) \sigma^{2}\left(\theta_{1}\right)+o\left(\left(\theta_{1}-\lambda^{-1}\left(c_{n}^{*}\right)\right)^{2}\right)+o\left(n^{-1}\right) .
\end{aligned}
$$

Using (5.2.12) we can write it also as follows

$$
\begin{aligned}
& \left(c_{n}-\lambda\left(\theta_{1}\right)\right)\left(\theta_{1}-\theta_{0}^{*}\right)+o\left(\left(c_{n}-\lambda\left(\theta_{1}\right)\right)^{2}\right)= \\
& \left(c_{n}^{*}-\lambda\left(\theta_{1}\right)\right)\left(\theta_{1}-\tilde{\theta}_{0}\right)+o\left(\left(c_{n}^{*}-\lambda\left(\theta_{1}\right)\right)^{2}\right)+o\left(n^{-1}\right) .
\end{aligned}
$$

Suppose $\lim _{i \rightarrow \infty}\left(c_{n_{i}}^{*}-\lambda\left(\theta_{1}\right)\right) \sigma\left(\theta_{1}\right) n_{i_{1}}^{\frac{3}{2}}=\infty$ for some subsequence $\left\{n_{i}\right\}$ then by (5.2.14) $\lim _{i \rightarrow \infty}\left(c_{n_{i}}-\lambda\left(\theta_{1}\right)\right) \sigma\left(\theta_{1}\right) n_{i}^{\frac{1}{2}}=-\infty$, which contradicts $\beta<1$. Moreover $\lim _{i \rightarrow \infty}\left(C_{n_{i}}^{*}-\lambda\left(\theta_{1}\right)\right) \sigma\left(\theta_{1}\right) n_{i}^{\frac{1}{2}}=-\infty$ also leads immediately to a contradiction, 
hence both $\left(c_{n}^{*}-\lambda\left(\theta_{1}\right)\right) \sigma\left(\theta_{1}\right) n^{\frac{1}{2}}=O(1)$ and $\left(c_{n}-\lambda\left(\theta_{1}\right)\right) \sigma\left(\theta_{1}\right) n^{\frac{1}{2}}=O(1)$ as $n \rightarrow \infty$. Now we can rewrite $(5.2 .14)$ as follows

$$
c_{n}-\lambda\left(\theta_{1}\right)=\left(\theta_{1}-\tilde{\theta}_{0}\right)\left(\theta_{1}-\theta_{0}^{*}\right)^{-1}\left(c_{n}^{*}-\lambda\left(\theta_{1}\right)\right)+o\left(n^{-1}\right) .
$$

Since $P_{\theta_{1}}\left(\bar{x}_{n} \in\left(c_{n}, c_{n}^{*}\right)\right) \leq \beta \leq P_{\theta_{1}}\left(\bar{x}_{n} \in\left[c_{n}, c_{n}^{*}\right]\right)$, the Berry-Esseen theorem implies

$$
\Phi\left(\left\{c_{n}^{*}-\lambda\left(\theta_{1}\right)\right\} \sigma\left(\theta_{1}\right)^{-1} n^{\frac{1}{2}}\right)-\Phi\left(\left\{c_{n}-\lambda\left(\theta_{1}\right)\right\} \sigma\left(\theta_{1}\right)^{-1} n^{\frac{1}{2}}\right)=\beta+O\left(n^{-\frac{1}{2}}\right) .
$$

Substituting $(5.2 .15)$ we obtain

(5.2.16) $\Phi\left(\left\{c_{n}^{*}-\lambda\left(\theta_{1}\right)\right\} \sigma\left(\theta_{1}\right)^{-1} n^{\frac{1}{2}}\right)-\Phi\left(\left(\theta_{1}-\tilde{\theta}_{0}\right)\left(\theta_{1}-\theta_{0}^{*}\right)^{-1}\left\{c_{n}^{*}-\lambda\left(\theta_{1}\right)\right\} \sigma\left(\theta_{1}\right)^{-1} n^{\frac{1}{2}}\right)=$

$$
=\beta+O\left(n^{-\frac{1}{2}}\right) \text {. }
$$

Define $\varepsilon_{\mathrm{n}}$ by

$$
\left\{c_{n}^{*}-\lambda\left(\theta_{1}\right)\right\} \sigma\left(\theta_{1}\right)^{-1} n^{\frac{1}{2}}=\gamma+\varepsilon_{n^{\prime}}
$$

where $\gamma>0$ is implicitely defined by

$$
\Phi(\gamma)-\Phi\left(\left(\theta_{1}-\tilde{\theta}_{0}\right)\left(\theta_{1}-\theta_{0}^{*}\right)^{-1} \gamma\right)=\beta
$$

(The function $h(t)=\Phi(t)-\Phi\left(\left(\theta_{1}-\tilde{\theta}_{0}\right)\left(\theta_{1}-\theta_{0}^{*}\right)^{-1} t\right)$ is continuous and strictly increasing on $(0, \infty)$ with $\lim _{t \downarrow 0} h(t)=0$ and $\lim _{t \rightarrow \infty} h(t)=1$; hence $\gamma$ is well defined.)

It is easily seen that $\lim _{n \rightarrow \infty} \varepsilon_{n}=0$. Moreover,

$$
\begin{aligned}
& \Phi\left(\gamma+\varepsilon_{\mathrm{n}}\right)-\Phi\left(\left(\theta_{1}-\tilde{\theta}_{0}\right)\left(\theta_{1}-\theta_{0}^{*}\right)^{-1}\left(\gamma+\varepsilon_{\mathrm{n}}\right)\right)= \\
& =\Phi(\gamma)-\Phi\left(\left(\theta_{1}-\tilde{\theta}_{0}\right)\left(\theta_{1}-\theta_{0}^{*}\right)^{-1} \gamma\right)+\varepsilon_{\mathrm{n}} \phi(\gamma)- \\
& -\left(\theta_{1}-\tilde{\theta}_{0}\right)\left(\theta_{1}-\theta_{0}^{*}\right)^{-1} \varepsilon_{\mathrm{n}} \phi\left(\left(\theta_{1}-\tilde{\theta}_{0}\right)\left(\theta_{1}-\theta_{0}^{*}\right)^{-1} \gamma\right)+0\left(\varepsilon_{\mathrm{n}}^{2}\right) \\
& =\beta+\left\{\phi(\gamma)-\left(\theta_{1}-\tilde{\theta}_{0}\right)\left(\theta_{1}-\theta_{0}^{*}\right)^{-1} \phi\left(\left(\theta_{1}-\tilde{\theta}_{0}\right)\left(\theta_{1}-\theta_{0}^{*}\right)^{-1} \gamma\right)\right\} \varepsilon_{\mathrm{n}}+O\left(\varepsilon_{\mathrm{n}}^{2}\right) .
\end{aligned}
$$

In combination with $(5.2 .16)$ we obtain $\varepsilon_{n}=O\left(n^{-\frac{1}{2}}\right)$ and thus 


$$
\left(c_{n}^{*}-\lambda\left(\theta_{1}\right)\right) \sigma\left(\theta_{1}\right)^{-1} n^{\frac{1}{2}}=\gamma+O\left(n^{-\frac{1}{2}}\right) .
$$

Let us now return to $\alpha_{n}^{+}\left(\beta, \theta_{1}\right)$ :

$$
\begin{aligned}
& \log \alpha_{n}^{+}\left(\beta, \theta_{1}\right)=-n I\left(\lambda^{-1}\left(c_{n}^{*}\right), \tilde{\theta}_{0}\right)-\frac{1}{2} \log n+O(1)= \\
& =-n I\left(\theta_{1}, \tilde{\theta}_{0}\right)+n^{\frac{1}{2}} \sigma\left(\theta_{1}\right) \gamma\left(\tilde{\theta}_{0}-\theta_{1}\right)-\frac{1}{2} \log n+O(1),
\end{aligned}
$$

where we have used $(5.2 .13)$ with $\theta_{0}^{*}$ replaced by $\tilde{\theta}_{0}$. This completes the proof of (5.2.1) in this case.

(b) Assume that sup $\theta_{0}<\theta_{1}$ and define $\theta_{0}^{*}$ by $\theta_{0}^{*}=\sup \theta_{0}$. Replacing $c_{n}^{*}$ by $\infty$ in the proof of case a(i) formula (5.2.1) easily follows.

(c) In a similar way one can deal with the case inf $\theta_{0}>\theta_{1}$.

So far we only assumed that $\theta_{1} \epsilon$ int $\theta_{1}$; now also assume that $I\left(\theta_{1}, \theta_{0}\right)<I\left(\theta_{0}\right)$. We wish to derive the same expansion for the LR test as for the MP test:

$$
\text { (5.2.17) } \log \alpha_{n}^{L R}\left(\beta, \theta_{1}\right)=-n I\left(\theta_{1}, \theta_{0}\right)+n^{\frac{1}{2}} B\left(\beta, \theta_{1}\right)-\frac{1}{2} \log n+O(1) \text {, }
$$

where $B\left(\beta, \theta_{1}\right)$ is defined in $(5.2 .2)$ and $(5.2 .3)$. We again distinguish the same cases as in the first part of the proof. Since proofs of the other cases are similar, we only prove case a(i): $\theta_{0}^{*}=\sup \left\{\theta_{0} \epsilon \theta_{0} ; \theta_{0}<\theta_{1}\right\}<\theta_{1}<$ $\inf \left\{\theta_{0} \epsilon \theta_{0}, \theta_{0}>\theta_{1}\right\}=\tilde{\theta}_{0}$ and $I\left(\theta_{1}, \theta_{0}^{*}\right)<I\left(\theta_{1}, \tilde{\theta}_{0}\right)$.

The LR test with power $\beta$ at $\theta_{1}$ rejects $H_{0}$ if $L\left(\bar{x}_{n}\right)>d_{n}$ and accepts $H_{0}$ if $L\left(\bar{x}_{n}\right)<d_{n^{\prime}}$ where $L(x)=\sup _{\theta \in \Theta}\{\theta x-\psi(\theta)\}-\sup _{\theta_{0} \in \theta_{0}}\left\{\theta_{0} x-\psi\left(\theta_{0}\right)\right\}$. Note that if $x \in \Lambda, L(x)>d_{n}$ is equivalent to $I\left(\lambda^{-1}(x), \theta_{0}\right)>d_{n}$. Define

$$
d_{n}^{\prime}>\lambda\left(\theta_{0}^{*}\right) \quad \text { by } \quad I\left(\lambda^{-1}\left(d_{n}^{\prime}\right), \theta_{0}^{*}\right)=d_{n}
$$

and

$$
d_{n}^{\prime \prime}<\lambda\left(\tilde{\theta}_{0}\right) \text { by } I\left(\lambda^{-1}\left(d_{n}^{\prime \prime}\right), \tilde{\theta}_{0}\right)=d_{n} \text {. }
$$

Since $E_{\theta_{1}} \phi_{n}^{L R}\left(\bar{x}_{n}\right) \geq \beta$ and $0<\beta<1, d_{n}^{\prime}$ and $d_{n}^{\prime \prime}$ exist for sufficiently large n. Again we define $\bar{\theta}$ by $I\left(\bar{\theta}, \theta_{0}^{*}\right)=I\left(\bar{\theta}^{n}, \tilde{\theta}_{0}\right)$. Since $d_{n}^{\prime \prime}>\lambda(\bar{\theta})$, this point plays no role (cf. $c_{n}^{*}$ in case a(i) for the MP test). Adapting the arguments leading to formula $(5.2 .8)$ to the present situation we find 
(5.2.19) $\quad d_{n}^{\prime}=\lambda\left(\theta_{1}\right)-\sigma\left(\theta_{1}\right) \Phi^{-1}(\beta) n^{-\frac{1}{2}}+O\left(n^{-1}\right)$.

(Note that the critical region of the LR test outside the interval $\left(\lambda\left(\theta_{0}^{*}\right), \lambda\left(\tilde{\theta}_{0}\right)\right)$ plays no role. $)$

Now consider $\alpha_{n}^{L R}\left(\beta, \theta_{1}\right)$. There exists a point $\theta_{0 n} \in \theta_{0}$ such that

$$
E_{\theta_{0 n}} \phi_{n}^{L R}\left(\bar{x}_{n}\right) \geq \frac{1}{2} \alpha_{n}^{L R}\left(\beta, \theta_{1}\right)
$$

We define $\theta_{O n}^{\prime}<\theta_{O n}<\theta_{O n}^{\prime \prime}$ by $I\left(\theta_{O n}^{\prime}, \theta_{0 n}\right)=I\left(\theta_{O n}^{\prime \prime}, \theta_{O n}\right)=d_{n}$. Because of $(5.2 .18),(5.2 .19)$ and $I\left(\theta_{1}, \theta_{0}\right)<I\left(\theta_{0}\right)$ the points $\theta_{0 n}^{\prime}$ and $\theta_{0 n}^{\prime \prime}$ exist and lie in a compact subset of int $\theta$ for sufficiently large $n$. Obviously

$$
P_{\theta_{O n}}\left(\bar{x}_{n} \leq \lambda\left(\theta_{O n}^{\prime}\right)\right)+P_{\theta_{O n}}\left(\bar{x}_{n} \geq \lambda\left(\theta_{O n}^{\prime \prime}\right)\right) \geq z_{2} \alpha_{n}^{L R}\left(\beta, \theta_{1}\right) .
$$

Assume

$$
P_{\theta_{0 \mathrm{n}}}\left(\overline{\mathrm{x}}_{\mathrm{n}} \leq \lambda\left(\theta_{\mathrm{On}}^{\prime}\right)\right) \geq \frac{1}{4} \alpha_{\mathrm{n}}^{\mathrm{LR}}\left(\beta, \theta_{1}\right)
$$

the case

$$
P_{\theta_{0 n}}\left(\bar{x}_{n} \geq \lambda\left(\theta_{0 n}^{\prime \prime}\right)\right) \geq \frac{1}{4} \alpha_{n}^{L R}\left(\beta, \theta_{1}\right)
$$

may be treated similarly. Then

$$
\begin{aligned}
& \alpha_{n}^{L R}\left(\beta, \theta_{1}\right) \leq 4 \exp \left\{-n I\left(\theta_{O n}^{\prime}, \theta_{O n}\right)\right\} \times \\
& \times \int_{(-\infty, 0]} \exp \left\{\left(\theta_{O n}{ }^{-\theta^{\prime}}{ }_{O n}\right) \sigma\left(\theta_{O n}^{\prime}\right) n^{\frac{1}{2}} y\right\} d \tilde{P}_{\theta_{O n}^{\prime}}^{n}(y) \\
& \leq 4 \exp \left\{-\mathrm{nI}\left(\lambda^{-1}\left(d_{n}^{\prime}\right), \theta_{0}^{*}\right)\right\} \times \\
& \times \int_{(-\infty, 0]} \exp \left\{\left(\theta_{0 n}{ }^{-\theta}{ }_{O n}^{\prime}\right) \sigma\left(\theta_{0 n}^{\prime}\right) n^{\frac{1}{2}} y\right\} d \tilde{P}_{\theta_{O n}^{\prime}}^{n}(y) .
\end{aligned}
$$

Since by the condition $I\left(\theta_{1}, \theta_{0}\right)<I\left(\theta_{0}\right) \quad \theta^{\prime}$ 'n remains in a compact subset of int $\theta$, the integral can be bounded by $c_{1} n^{-\frac{1}{2}}$ for some positive constant $c_{1}$ in view of the Berry-Esseen theorem. Hence, using (5.2.19),

$$
\begin{aligned}
& \alpha_{n}^{L R}\left(\beta, \theta_{1}\right) \leq 4 c_{1} n^{-\frac{1}{2}} \exp \left\{-n I\left(\lambda^{-1}\left(d_{n}^{\prime}\right), \theta_{0}^{*}\right)\right\} \\
& \leq \exp \left\{-n I\left(\theta_{1}, \theta_{0}^{*}\right)+n^{\frac{1}{2}}\left(\theta_{1}-\theta_{0}^{*}\right) \sigma\left(\theta_{1}\right) \Phi^{-1}(\beta)-\frac{1}{2} \log n+c_{2}\right\}
\end{aligned}
$$


for some positive constant $c_{2}$.

The reverse inequality is easy since $\alpha_{n}^{L R}\left(\beta, \theta_{1}\right) \geq \alpha_{n}^{+}\left(\beta, \theta_{1}\right)$. Thus we have proved formula (5.2.17) for the case $a(i)$.

Finally we translate our results concerning $\alpha_{n}^{+}\left(\beta, \theta_{1}\right)$ and $\alpha_{n}^{L R}\left(\beta, \theta_{1}\right)$ in statements concerning $N^{+}\left(\alpha, \beta, \theta_{1}\right)$ and $N^{L R}\left(\alpha, \beta, \theta_{1}\right)$. Since by (5.1.3)

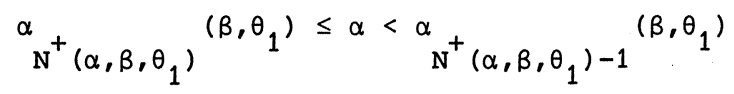

easy calculations yield that for $\alpha \rightarrow 0$

$$
\begin{aligned}
N^{+}\left(\alpha, \beta, \theta_{1}\right) & =\frac{-\log \alpha}{I\left(\theta_{1}, \theta_{0}\right)}+\frac{B\left(\beta, \theta_{1}\right)}{I\left(\theta_{1}, \theta_{0}\right)}\left\{\frac{-\log \alpha}{I\left(\theta_{1}, \theta_{0}\right)}\right\}^{\frac{1}{2}} \\
& -\frac{\frac{1}{2}}{I\left(\theta_{1}, \theta_{0}\right)} \log \left\{\frac{-\log \alpha}{I\left(\theta_{1}, \theta_{0}\right)}\right\}+O(1)
\end{aligned}
$$

and the same expansion holds for $N^{L R}\left(\alpha, \beta, \theta_{1}\right)$.

Hence the LR test is deficient in the sense of Bahadur at $\theta_{1}$ of order $O(1)$ as $\alpha \rightarrow 0$.

In the pronf of formula (5.2.1) we only have used that $\theta_{1} \epsilon$ int $\theta_{1}$ and did not need the condition $I\left(\theta_{1}, \theta_{0}\right)<I\left(\theta_{0}\right)$ or the fact that $\theta_{0}$ lies in a compact subset of int $\theta$. Hence we also proved

LEMMA 5.2.2. Let $\theta_{0}$ be an arbitrary subset of $\theta$ and let $\theta_{1}=\theta-\theta_{0}$. If $\theta \in$ int $\theta_{1}$

$$
\log \alpha_{n}^{+}(\beta, \theta)=-n I\left(\theta, \theta_{0}\right)+n^{\frac{1}{2}} B(\beta, \theta)-\frac{1}{2} \log n+O(1),
$$

where $\mathrm{B}(\beta, \theta)$ is defined as in $(5.2 .2)$ and $(5.2 .3)$.

We now consider a second situation where the boundary of the critical region of the LR test stays away from the boundary of the parameter space.

THEOREM 5.2.3. If $\theta_{1}$ is contained in a compact subset of int $\theta$, then for each $\theta \in$ int $\theta_{1}$ the LR test is deficient in the sense of Bahadur at $\theta$ of order $O(1)$ as $\alpha \rightarrow 0$.

PROOF. Let $\theta_{1} \in$ int $\theta_{1}$ and choose $\beta \in(0,1)$. Let $\phi_{n}^{L R}$ denote the LR test with 
power $\beta$ at $\theta_{1}$. Then there exists a point $\theta_{0 n} \in \operatorname{cl} \theta_{0} \wedge\left[\theta^{\prime}, \theta^{\prime \prime}\right]$ such that

$$
\mathrm{E}_{\theta_{\text {On }}}\left\{\phi_{\mathrm{n}}^{\mathrm{LR}}\left(\overline{\mathrm{x}}_{\mathrm{n}}\right) 1_{\left[\lambda\left(\theta^{\prime}\right), \lambda\left(\theta^{\prime}\right)\right]}\left(\overline{\mathrm{x}}_{\mathrm{n}}\right)\right\} \geq \frac{1}{2} \alpha_{n}^{\mathrm{LR}}\left(\beta, \theta_{1}\right),
$$

where $\theta^{\prime}=\inf \theta_{1}$ and $\theta^{\prime \prime}=\sup \theta_{1}$. Define $\theta_{\text {On }}^{\prime}<\theta_{\text {On }}<\theta_{\text {On }}^{\prime \prime}$ by $I\left(\theta_{O_{n}}^{\prime} \theta_{0 n}\right)=$ $I\left(\theta_{O n}, \theta_{O n}\right)=d_{n}$; at least one of the points $\theta_{O n}^{\prime}$ and $\theta_{O n}^{\prime \prime}$ exists and lies in $\left[\theta^{\prime}, \theta^{\prime}\right]$, hence in a compact subset of int $\theta$. By the same line of arguments that we used in the proof of theorem 5.2.1 following (5.2.19) it can be shown that

$$
\log \alpha_{n}^{L R}\left(\beta, \theta_{1}\right)=-n I\left(\theta_{1}, \theta_{0}\right)+n^{\frac{1}{2}} B\left(\beta, \theta_{1}\right)-\frac{1}{2} \log n+O(1)
$$

Together with lemma 5.2.2 this again implies that the LR test is deficient in the sense of Bahadur at $\theta_{1}$ of order $O(1)$ as $\alpha+0$.

For an arbitrary null hypothesis we introduce the following assumptions (cf. section 3.5).

ASSUMPTION A1. The LR test satisfies:

$$
\frac{\sup _{\theta_{0} \epsilon \Theta_{0} \wedge \mathrm{K}} \mathrm{E}_{\theta_{0}} \phi_{\mathrm{n}}^{\mathrm{LR}}\left(\overline{\mathrm{x}}_{\mathrm{n}}\right)}{\sup _{\theta \in \Theta_{0}} \mathrm{E}_{\theta} \phi_{\mathrm{n}}^{\mathrm{LR}}\left(\overline{\mathrm{x}}_{\mathrm{n}}\right)} \geq \varepsilon
$$

for all $\mathrm{n}$, some compact subset $\mathrm{K}$ of int $\theta$ and some positive $\varepsilon$.

ASSUMPTION A2. $\theta$ satisfies $0<I\left(\theta_{,} \theta_{0}\right)<I\left(\theta_{0} \wedge \mathrm{K}\right)$.

THEOREM 5.2.4. Under the assumptions A1 and A2 the LR test is deficient in the sense of Bahadur at $\theta$ of order $O(1)$ as $\alpha \rightarrow 0$ for all $\theta \epsilon$ int $\theta_{1}$.

PROOF. A slight modification of the proof of theorem 5.2.1 yields the result.

As in chapter II we can also introduce moment conditions on the probability distribution of the random variables.

THEOREM 5.2.5. Let the variance $\sigma^{2}(\theta)$ of $\mathrm{x}$ be bounded away from zero and let the absolute third central moment of $\mathrm{x}$ be bounded above for all $\theta \epsilon$ int $\theta$, then for each $\theta \in$ int $\theta_{1}$ the LR test is deficient in the sense of Bahadur at $\theta$ of order $O(1)$ as $\alpha \rightarrow 0$. 
PROOF. Let $\theta_{1} \in$ int $\theta_{1}$ and let $\phi_{n}^{L R}$ be the LR test with power $\beta$ at $\theta_{1}$. In view of lemma 5.2.2 we only have to prove that

$$
\alpha_{n}^{L R}\left(\beta, \theta_{1}\right) \leq \exp \left\{-n I\left(\theta_{1}, \theta_{0}\right)+n^{\frac{1}{2}} B\left(\beta, \theta_{1}\right)-\frac{1}{2} \log n+c\right\}
$$

for some positive constant $c$, where $B\left(\beta, \theta_{1}\right)$ is defined in (5.2.2) and (5.2.3). The boundedness of the absolute third central moment of $x$ implies that $\sigma^{2}(\theta)$ is also bounded above on int $\theta$. Moreover, if $\theta \in \theta$ is a boundary point of $\theta$ and $\lambda(\theta)$ is finite, then the variance and the third central moment at $\theta$ are also finite. Note that there exist constants $c_{1}$ and $c_{2}$ such that for all $\theta, \xi \in \theta$ with $\theta \neq \xi$ and $\lambda(\theta)$ finite we have

$$
0<c_{1} \leq I(\theta, \xi)(\theta-\xi)^{-2} \leq c_{2}<\infty
$$

(for $\theta, \xi \in$ int $\theta$ use lemma 2.2.2 (iii) and the boundedness of $\sigma^{2}(\theta$ ); for possible boundary points use the continuity of the functions $\psi$ and $\lambda$ ).

There exists a point $\theta_{\text {on }} \epsilon \theta_{0}$ such that

$$
E_{\theta_{0 n}} \phi_{n}^{L R}\left(\bar{x}_{n}\right) \geq \frac{1}{2} \frac{L R}{L R}\left(\beta, \theta_{1}\right)
$$

We define $\theta_{0 n}^{\prime}<\theta_{0 n}<\theta_{O n}^{\prime \prime}$ by $I\left(\theta_{O n}^{\prime}{ }^{\prime} \theta_{0 n}\right)=I\left(\theta_{0 n}^{\prime \prime} \theta_{O n}\right)=d_{n}$; at least one of the points $\theta_{\text {On }}^{\prime}$ and $\theta_{\text {On }}$ exists. We can estimate for instance

$$
P_{\theta_{0 n}}\left(\bar{x}_{n} \leq \lambda\left(\theta_{O n}^{\prime}\right)\right)
$$

as follows:

$$
\begin{aligned}
& P_{\theta_{O n}}\left(\bar{x}_{n} \leq \lambda\left(\theta_{O n}^{\prime}\right)\right)=\exp \left\{-n I\left(\theta_{O_{n}{ }^{\prime}} \theta_{O n}\right)\right\} \times \\
& \times \sum_{j=0}^{\infty} \int_{\left(-(j-1) n^{-\frac{1}{2}},-j n^{-\frac{1}{2}}\right]} \exp \left\{n^{\frac{1}{2}}\left(\theta_{0 n}-\theta_{0 n}^{\prime}\right) \sigma\left(\theta_{0 n}^{\prime}\right) y\right\} d \tilde{P}_{\theta_{0 n}^{\prime}}^{n}(y) \\
& \leq \exp \left\{-n I\left(\theta_{0 n^{\prime}}^{\prime} \theta_{O n}\right)\right\} \sum_{j=0}^{\infty} \exp \left\{-\left(\theta_{0 n}{ }^{-\theta_{0 n}^{\prime}}\right) \sigma\left(\theta_{O n}^{\prime}\right) j\right\} \tilde{P}_{\theta_{0 n}^{\prime}}^{n}\left(\left(-(j-1) n^{-\frac{1}{2}},-j n^{-\frac{1}{2}}\right]\right) \text {. }
\end{aligned}
$$

Since the third absolute central moment is bounded above we can apply BerryEsseen's theorem to ensure that

$$
\tilde{\mathrm{P}}_{\theta}^{\mathrm{n}}\left(\left(-(j-1) \mathrm{n}^{-\frac{1}{2}},-j \mathrm{n}^{-\frac{1}{2}}\right]\right) \leq c_{3} \mathrm{n}^{-\frac{1}{2}}
$$

for some positive constant $c_{3}$. Hence 


$$
P_{\theta_{0 n}}\left(\bar{x}_{n} \leq \lambda\left(\theta_{O n}^{\prime}\right)\right) \leq c_{3} n^{-\frac{1}{2}} \frac{\exp \left\{-n I\left(\theta_{O n}^{\prime}, \theta_{O n}\right)\right\}}{1-\exp \left\{-\left(\theta_{0 n}{ }^{-\theta_{O n}^{\prime}}\right) \sigma\left(\theta_{O n}^{\prime}\right)\right\}} .
$$

Since $I\left(\theta_{0 n}^{\prime}, \theta_{0 n}\right) \geq \frac{1}{2} I\left(\theta_{1}, \theta_{0}\right)$ for sufficiently large $n,\left(\theta_{0 n}-\theta_{0 n}^{\prime}\right)^{2} \geq$ $\mathrm{c}_{2}^{-1} \mathrm{I}\left(\theta_{0 n^{\prime}}^{\prime} \theta_{0 n}\right)$ and $\sigma\left(\theta_{0 n}^{\prime}\right)$ is bounded away from zero, it holds that $\lim \inf _{n \rightarrow \infty}\left(\theta_{0 n}{ }^{-\theta}{ }_{O n}\right) \sigma\left(\theta_{O n}^{\prime}\right)>0$ and hence

$$
P_{\theta_{0 n}}\left(\bar{x}_{n} \leq \lambda\left(\theta_{0 n}^{\prime}\right)\right) \leq c_{4} n^{-\frac{1}{2}} \exp \left\{-n I\left(\theta_{O n}^{\prime}, \theta_{O n}\right)\right\}
$$

for some positive constant $\mathrm{c}_{4}$.

The remaining part of the proof is similar to that of theorem 5.2.1.

In all previous theorems in this section assumptions were introduced to guarantee that the LR test is deficient in the sense of Bahadur of order $O(1)$. In the general case we have the following theorem (cf. example $5.1 .2):$

THEOREM 5.2.6. Let $\theta_{0}$ be an arbitrary subset of $\theta$ and $\theta_{1}=\theta-\theta_{0}$. For every $\theta \in$ int $\theta_{1}$ the LR test is deficient in the sense of Bahadur at $\theta$ of order $O\left(\log \mathrm{N}^{+}(\alpha, \beta, \theta)\right)$ as $\alpha \rightarrow 0$.

PROOF. Let $\theta_{1} \in$ int $\theta_{1}$ and $0<\beta<1$. Since in lemma 5.2 .2 an expansion for $\alpha_{n}^{+}\left(\beta, \theta_{1}\right)$ is given, we only have to consider the LR test with power $\beta$ at $\theta_{1}$. We use the general result of lemma 2.3.1:

$$
\alpha_{n}^{L R}\left(\beta, \theta_{1}\right) \leq 5 \exp \left(-n d_{n}\right)
$$

It is easily verified (cf. $(5.2 .18)$ and $(5.2 .19)$ ) that

$$
d_{n}=I\left(\theta_{1}, \theta_{0}\right)+n^{-\frac{1}{2}} B\left(\beta, \theta_{1}\right)+O\left(n^{-1}\right),
$$

where $B\left(B, \theta_{1}\right)$ is defined in $(5.2 .2)$ and $(5.2 .3)$. Hence

$$
\alpha_{n}^{L R}\left(\beta, \theta_{1}\right) \leq 5 \exp \left\{-n I\left(\theta_{1}, \theta_{0}\right)+n^{\frac{1}{2}} B\left(\beta, \theta_{1}\right)+c_{1}\right\}
$$

for some positive constant $c_{1}$. Together with lemma 5.2.2 this implies

$$
N^{L R}\left(\alpha, \beta, \theta_{1}\right)-N^{+}\left(\alpha, \beta, \theta_{1}\right) \leq c_{2} \operatorname{log~N^{+}}\left(\alpha, \beta, \theta_{1}\right)
$$

for some positive constant $\mathrm{c}_{2}$. 
5.3. THE k-DIMENSIONAL CASE

In many testing problems for one-parameter exponential families the LR test is deficient in the sense of Bahadur of order $O(1)$. However, in testing problems for $k$-parameter exponential families with $k \geq 2$ this is quite exceptional.

EXAMPLE 5.3.1. Let $x_{1}, x_{2}, \ldots$ be i.i.d. random two-dimensional vectors with a normal $N\left(\left(\mu_{1}, \mu_{2}\right) ; I_{2}\right)$ distribution. Consider the testing problem $\mathrm{H}_{0}:\left(\mu_{1}, \mu_{2}\right)=(0,0)$ against $\mathrm{H}_{1}:\left(\mu_{1}, \mu_{2}\right) \neq(0,0)$.

We investigate the Bahadur deficiency of the LR test at $(0,1)$ with respect to the MP test for $\mathrm{H}_{0}$ against $\mathrm{H}_{1}^{*}:\left(\mu_{1}, \mu_{2}\right)=(0,1)$. The MP test has the following form: reject $H_{0}$ if $\bar{x}_{n}^{(2)}>c_{n}$ where $\bar{x}_{n}^{(j)}=n^{-1} \sum_{i=1}^{n} x_{i}^{(j)}$, $j=1,2$. The LR test rejects $H_{0}$ if $\left(\bar{x}_{n}^{(1)}\right)^{2}+\left(\bar{x}_{n}^{(2)}\right)^{2}>d_{n}$. We choose $c_{n}$ and $d_{n}$ such that the power of both tests at $(0,1)$ equals $\beta, 0<\beta<1$. This implies that $c_{n}=1-\Phi^{-1}(\beta) n^{-\frac{1}{2}}$. Consequently

$$
\begin{aligned}
& \alpha_{n}^{+}(\beta,(0,1))=P_{(0,0)}\left(\bar{x}_{n}^{(2)}>1-\Phi^{-1}(\beta) n^{-\frac{1}{2}}\right) \\
& =\frac{\exp \left\{-\frac{1}{2}\left(n^{\frac{1}{2}}-\Phi^{-1}(\beta)\right)^{2}\right\}}{(2 \pi)^{\frac{1}{2}}\left\{n^{\frac{1}{2}}-\Phi^{-1}(\beta)\right\}}(1+O(1))
\end{aligned}
$$

and hence

$$
\log \alpha_{n}^{+}(\beta,(0,1))=-\frac{1}{2} n+\Phi^{-1}(\beta) n^{\frac{1}{2}}-\frac{1}{2} \log n+O(1)
$$

as $\mathrm{n} \rightarrow \infty$. Since

$$
\begin{aligned}
& P_{(0,1)}\left(\left(\overline{\mathrm{x}}_{\mathrm{n}}^{(1)}\right)^{2}+\left(\overline{\mathrm{x}}_{\mathrm{n}}^{(2)}\right)^{2}>\left\{1-\Phi^{-1}(\beta) \mathrm{n}^{-\frac{1}{2}}\right\}^{2}\right)> \\
& >P_{(0,1)}\left(\overline{\mathrm{x}}_{\mathrm{n}}^{(2)}>1-\Phi^{-1}(\beta) \mathrm{n}^{-\frac{1}{2}}\right)=\beta,
\end{aligned}
$$

it follows that $d_{n}>\left\{1-\Phi^{-1}(\beta) n^{-\frac{1}{2}}\right\}^{2}$ and hence

$$
\begin{aligned}
& \alpha_{n}^{L R}(\beta,(0,1)) \leq P_{(0,0)}\left(\left(\bar{x}_{n}^{(1)}\right)^{2}+\left(\bar{x}_{n}^{(2)}\right)^{2}>\left\{1-\Phi^{-1}(\beta) n^{-\frac{1}{2}}\right\}^{2}\right) \\
& \leq \exp \left\{-\frac{1}{2} n+\Phi^{-1}(\beta) n^{\frac{1}{2}}-\frac{1}{2}\left(\Phi^{-1}(\beta)\right)^{2}\right\} .
\end{aligned}
$$


On the other hand it is easily verified that there exists a positive constant A such that $d_{n} \leq 1-2 \Phi^{-1}(B) n^{-\frac{1}{2}}+A n^{-1}$. Therefore

$$
\begin{aligned}
& \alpha_{n}^{L R}(\beta,(0,1)) \geq P_{(0,0)}\left(\left(\bar{x}_{n}^{(1)}\right)^{2}+\left(\bar{x}_{n}^{(2)}\right)^{2}>1-2 \Phi^{-1}(\beta) n^{-\frac{1}{2}}+A n^{-1}\right) \\
& =\exp \left\{-\frac{1}{2} n+\Phi^{-1}(\beta) n^{\frac{1}{2}}-\frac{1}{2} A\right\}
\end{aligned}
$$

and hence

$$
\log \alpha_{n}^{L R}(\beta,(0,1))=-\frac{1}{2} n+\Phi^{-1}(\beta) n^{\frac{1}{2}}+O(1)
$$

as $n \rightarrow \infty$. It follows that

$$
\mathbf{N}^{+}(\alpha, \beta,(0,1))=-2 \log \alpha+2 \Phi^{-1}(\beta)\{-2 \log \alpha\}^{\frac{1}{2}}-\log (-2 \log \alpha)+O(1)
$$

and

$$
\mathrm{N}^{\mathrm{LR}}(\alpha, \beta,(0,1))=-2 \log \alpha+2 \Phi^{-1}(\beta)\{-2 \log \alpha\}^{\frac{1}{2}}+O(1)
$$

for $\alpha \rightarrow 0$. Thus

$$
N^{L R}(\alpha, \beta,(0,1))=N^{+}(\alpha, \beta,(0,1))+\log N^{+}(\alpha, \beta,(0,1))+O(1)
$$

for $\alpha \rightarrow 0$. So, even for a very smooth testing problem we obtain that the LR test is not deficient in the sense of Bahadur of order $O(1)$.

Although there are examples of testing problems for $k$-parameter exponential families, where the LR test is deficient in the sense of Bahadur of order $O(1)$ (e.g. tèsting $\mathrm{H}_{0}: \sigma^{2} \leq \sigma_{0}^{2}$ against $\mathrm{H}_{1}: \sigma^{2}>\sigma_{0}^{2}$ in normal $\mathrm{N}\left(\mu, \sigma^{2}\right)$ families), it turns out that under rather general conditions its deficiency is of order $O\left(\log \mathrm{N}^{+}(\alpha, \beta, \theta)\right)$. Our first theorem deals with the easiest case: a simple hypothesis.

THEOREM 5.3.1. Let $\mathrm{x}_{1}, \mathrm{x}_{2}, \ldots$ be $i . i . d$. random $\mathrm{k}$-vectors from an exponential family. Consider the testing problem $\mathrm{H}_{0}: \theta=\theta_{0}$ against $\mathrm{H}_{1}: \theta \neq \theta_{0}$ where $\theta_{0} \in$ int $\theta$. Then for every $\theta \in\left\{\theta_{i} I\left(\theta_{1}, \theta_{0}\right)<I\left(\theta_{0}\right)\right\}$ the LR test is deficient in the sense of Bahadur at $\theta$ of order $0\left(\log \mathrm{N}^{+}(\alpha, \beta, \theta)\right)$ as $\alpha \rightarrow 0$.

PROOF. Let $\theta_{1} \in\left\{\theta_{i} I\left(\theta_{,} \theta_{0}\right)<I\left(\theta_{0}\right)\right\}$. In fact we will prove that there exists a constant $C=C\left(\beta, \theta_{1}\right)$ such that

$$
\mathbf{N}^{L R}\left(\alpha, \beta, \theta_{1}\right)-\mathbf{N}^{+}\left(\alpha, \beta, \theta_{1}\right) \leq \frac{1}{2}(k-1)\left\{I\left(\theta_{1}, \theta_{0}\right)\right\}^{-1} \log N^{+}\left(\alpha, \beta, \theta_{1}\right)+C .
$$


As usual we compare the LR test with the MP test for the testing problem $H_{0}: \theta=\theta_{0}$ against $H_{1}^{*}: \theta=\theta_{1}$. The latter test has the following form:

$$
\phi_{n}^{+}(x)=\left\{\begin{array}{lll}
1 & \text { if }\left(\theta_{1}-\theta_{0}\right) x^{>} c_{n}^{+}
\end{array}\right.
$$

where $c_{n}^{+}$is such that $E_{\theta_{1}} \phi_{n}^{+}\left(\bar{x}_{n}\right)=\beta(0<\beta<1)$. It is easy to see that $c_{n}^{+}$satisfies

$$
c_{n}^{+}=\left(\theta_{1}-\theta_{0}\right) \cdot \lambda\left(\theta_{1}\right)-\left\{\left(\theta_{1}-\theta_{0}\right) \cdot \Sigma_{\theta_{1}}\left(\theta_{1}-\theta_{0}\right)\right\}^{\frac{1}{2}} \Phi^{-1}(\beta) n^{-\frac{1}{2}}+O\left(n^{-1}\right)
$$

Since $I\left(\theta_{1}, \theta_{0}\right)<I\left(\theta_{0}\right)$ and $\left(\theta_{1}-\theta_{0}\right) \cdot \lambda\left(\theta_{0}+\gamma\left(\theta_{1}-\theta_{0}\right)\right)$ is a strictly increasing function of $\gamma$, there exists for sufficiently large $n$ a unique $\gamma_{n}>0$ such that $c_{n}^{+}=\left(\theta_{1}-\theta_{0}\right) \cdot \lambda\left(\theta_{0}+\gamma_{n}\left(\theta_{1}-\theta_{0}\right)\right)$. Let $\xi_{n}=\theta_{0}+\gamma_{n}\left(\theta_{1}-\theta_{0}\right)$.

The LR test for our testing problem has the following form:

$$
\phi_{\mathrm{n}}^{\mathrm{LR}}(\mathrm{x})=\left\{\left\{_{0}^{1} \text { if } \sup _{\theta \in \Theta}\left\{\theta^{\prime} \mathrm{x}-\psi(\theta)\right\}-\left\{\theta_{0}^{\prime} \mathrm{x}-\psi\left(\theta_{0}\right)\right\}<\mathrm{d}_{\mathrm{n}},\right.\right.
$$

where $a_{n}$ is such that $E_{\theta_{1}} \phi_{n}^{L R}\left(\bar{x}_{n}\right)=\beta$. By considering the test $\tilde{\phi}_{n}$ defined by

$$
\tilde{\phi}_{n}(x)=\left\{\int_{0}^{1} \text { if } \sup _{\theta \in \Theta}\left\{\theta^{\prime} x-\psi(\theta)\right\}-\left\{\theta_{0}^{\prime} x-\psi\left(\theta_{0}\right)\right\}<I\left(\xi_{n}, \theta_{0}\right),\right.
$$

we conclude that $d_{n} \geq I\left(\xi_{n}, \theta_{0}\right)$, since $\sup _{\theta \in \theta^{\prime}}\left\{\theta^{\prime} x-\psi(\theta)\right\} \geq \xi_{n}^{\prime} x-\psi\left(\xi_{n}\right)$ and therefore the critical region of $\tilde{\phi}_{n}$ contains the critical region of $\phi_{n}^{+}$. Hence we obtain for $\alpha_{n}^{L R}\left(\beta, \theta_{1}\right)$ the following inequalities:

$$
\begin{aligned}
& \alpha_{n}^{L R}\left(\beta, \theta_{1}\right) \leq P_{\theta_{0}}\left(\sup _{\theta \in \theta^{\prime}}\left\{\theta^{\prime} \bar{x}_{n}-\psi(\theta)\right\}-\left\{\theta_{0}^{\prime} \bar{x}_{n}-\psi\left(\theta_{0}\right)\right\} \geq I\left(\xi_{n}, \theta_{0}\right)\right) \\
& \leq c_{1} n^{(k-2) / 2} \exp \left\{-n I\left(\xi_{n}, \theta_{0}\right)\right\}
\end{aligned}
$$

for some constant $c_{1}$, where the last inequality is an application of theorem 3.2.1. Since $\left(\theta_{1}-\theta_{0}\right) \cdot \lambda\left(\xi_{n}\right)=c_{n}^{+}=\left(\theta_{1}-\theta_{0}\right) \cdot \lambda\left(\theta_{1}\right)-\left\{\left(\theta_{1}-\theta_{0}\right) \cdot \Sigma_{\theta_{1}}\left(\theta_{1}-\theta_{0}\right)\right\}^{\frac{1}{2} \Phi^{-1}}(\beta) n^{-\frac{1}{2}}+$ $O\left(n^{-1}\right), I\left(\xi_{n}, \theta_{0}\right)=I\left(\theta_{1}, \theta_{0}\right)+I\left(\xi_{n}, \theta_{1}\right)+\left(\theta_{1}-\theta_{0}\right) \cdot\left\{\lambda\left(\xi_{n}\right)-\lambda\left(\theta_{1}\right)\right\}$ and $\left\|\xi_{n}-\theta_{1}\right\|=$ $O\left(n^{-\frac{1}{2}}\right)$, it holds that

$$
I\left(\xi_{n}, \theta_{0}\right)=I\left(\theta_{1}, \theta_{0}\right)-\left\{\left(\theta_{1}-\theta_{0}\right) \cdot \Sigma_{\theta_{1}}\left(\theta_{1}-\theta_{0}\right)\right\}^{\frac{1}{2}} \Phi^{-1}(\beta) n^{-\frac{1}{2}}+O\left(n^{-1}\right)
$$

This implies that 
110

$$
\begin{aligned}
& \alpha_{n}^{L R}\left(\beta, \theta_{1}\right) \leq c_{2} n^{(k-2) / 2} \exp \left\{-n I\left(\theta_{1}, \theta_{0}\right)+\right. \\
&\left.+n^{\frac{1}{2}}\left\{\left(\theta_{1}-\theta_{0}\right) \cdot \Sigma_{\theta_{1}}\left(\theta_{1}-\theta_{0}\right)\right\}^{\frac{3}{2}} \Phi^{-1}(\beta)\right\}
\end{aligned}
$$

for some constant $c_{2}$.

on the other hand

$$
\begin{aligned}
& \alpha_{n}^{+}\left(\beta, \theta_{1}\right) \geq P_{\theta_{0}}\left(\left(\theta_{1}-\theta_{0}\right) \cdot \bar{x}_{n}>\left(\theta_{1}-\theta_{0}\right) \cdot \lambda\left(\xi_{n}\right)\right) \\
& \geq \int_{\left(\xi_{n}-\theta_{0}\right){ }^{\prime}\left(x-\lambda\left(\xi_{n}\right)\right)>0} \exp \left\{n\left(\theta_{0}-\xi_{n}\right) \cdot\left(x-\lambda\left(\xi_{n}\right)\right)-n I\left(\xi_{n}, \theta_{0}\right)\right\} d \bar{P}_{\xi_{n}}^{-n}(x) .
\end{aligned}
$$

Substituting

$$
y=\left\{\left(\xi_{n}-\theta_{0}\right) \cdot \Sigma_{\xi_{n}}\left(\xi_{n}-\theta_{0}\right)\right\}^{-\frac{1}{2}}\left(\xi_{n}-\theta_{0}\right) \cdot\left(x-\lambda\left(\xi_{n}\right)\right) n^{\frac{1}{2}}
$$

we obtain

$$
\alpha_{n}^{+}\left(\beta, \theta_{1}\right) \geq \int_{(0, \infty)} \exp \left\{-n I\left(\xi_{n}, \theta_{0}\right)-n^{\frac{1}{2}}\left\{\left(\xi_{n}-\theta_{0}\right) \cdot \Sigma_{\xi_{n}}\left(\xi_{n}-\theta_{0}\right)\right\}^{\frac{1}{2}} y\right\} d \tilde{P}_{\xi_{n}}^{n}(y)
$$

where

$$
\tilde{\mathrm{P}}_{\xi_{\mathrm{n}}}^{\mathrm{n}}(\mathrm{B})=P_{\xi_{\mathrm{n}}}\left(\left\{\left(\xi_{\mathrm{n}}-\theta_{0}\right) \cdot \Sigma_{\xi_{n}}\left(\xi_{\mathrm{n}}-\theta_{0}\right)\right\}^{-\frac{1}{2}}\left(\xi_{n}-\theta_{0}\right) \cdot\left(\overline{\mathrm{x}}_{\mathrm{n}}-\lambda\left(\xi_{\mathrm{n}}\right)\right) \mathrm{n}^{\frac{1}{2}} \in \mathrm{B}\right)
$$

for all Borel sets B. The Berry-Esseen theorem ensures the existence of a constant $c_{3}>0$ such that the last integral is at least equal to $c_{3} n^{-\frac{1}{2}}$ $\exp \left\{-n I\left(\xi_{n}, \theta_{0}\right)\right\}$. Hence application of (5.3.1) yields

$$
\alpha_{n}^{+}\left(\beta, \theta_{1}\right) \geq c_{4} n^{-\frac{1}{2}} \exp \left\{-n I\left(\theta_{1}, \theta_{0}\right)+n^{\frac{1}{2}}\left\{\left(\theta_{1}-\theta_{0}\right) \Sigma_{\theta_{1}}\left(\theta_{1}-\theta_{0}\right)\right\}^{\frac{1}{2}} \Phi^{-1}(\beta)\right\} .
$$

It follows that

$$
\begin{aligned}
& I\left(\theta_{1}, \theta_{0}\right) N^{L R}\left(\alpha, \beta, \theta_{1}\right) \leq \\
& \leq-\log \alpha+\left\{\left(\theta_{1}-\theta_{0}\right) \cdot \Sigma_{\theta_{1}}\left(\theta_{1}-\theta_{0}\right)\right\}^{\frac{1}{2}} \Phi^{-1}(\beta)\left\{\frac{-\log \alpha}{I\left(\theta_{1}, \theta_{0}\right)}\right\}^{\frac{1}{2}} \\
& +\left(\frac{k-2}{2}\right) \log \left\{\frac{-\log \alpha}{I\left(\theta_{1}, \theta_{0}\right)}\right\}+c_{5}
\end{aligned}
$$

and 


$$
\begin{aligned}
& I\left(\theta_{1}, \theta_{0}\right) N^{+}\left(\alpha, \beta, \theta_{1}\right) \geq \\
& \geq-\log \alpha+\left\{\left(\theta_{1}-\theta_{0}\right) \cdot \Sigma_{\theta_{1}}\left(\theta_{1}-\theta_{0}\right)\right\}^{\frac{1}{2}} \Phi^{-1}(\beta)\left\{\frac{-\log \alpha}{I\left(\theta_{1}, \theta_{0}\right)}\right\}^{\frac{1}{2}}- \\
& -\frac{1}{2} \log \left\{\frac{-\log \alpha}{I\left(\theta_{1}, \theta_{0}\right)}\right\}+c_{6}
\end{aligned}
$$

for some constants $c_{5}$ and $c_{6}$ and therefore

$$
N^{L R}\left(\alpha, \beta, \theta_{1}\right)-N^{+}\left(\alpha, \beta, \theta_{1}\right) \leq \frac{1}{2}(k-1)\left\{I\left(\theta_{1}, \theta_{0}\right)\right\}^{-1} \log N^{+}\left(\alpha, \beta, \theta_{1}\right)+c_{7}
$$

for some constant $c_{7} \cdot \square$

As our next step we consider a null hypothesis contained in a compact subset of int $\theta$.

THEOREM 5.3.2. Let $\theta_{0}$ be a subset of a compact subset of int $\theta$ and let $\theta_{1}=\theta-\theta_{0}$. Then for every $\theta \in$ int $\theta_{1}$ with $I\left(\theta, \theta_{0}\right)<I\left(\theta_{0}\right)$ the LR test is deficient in the sense of Bahadur at $\theta$ of order $O\left(\log \mathrm{N}^{+}(\alpha, \beta, \theta)\right)$ as $\alpha \rightarrow 0$.

PROOF. Let $\theta_{1} \in$ int $\theta_{1}$ with $I\left(\theta_{1}, \theta_{0}\right)<I\left(\theta_{0}\right)$ and let $c_{i}(i=1, \ldots 29)$ denote constants with $0<c_{i}<\infty(i=1, \ldots 29)$.

The proof is given in several steps:

A. We show that

(5.3.2) $\quad \alpha_{n}^{L R}\left(\beta, \theta_{1}\right) \leq c_{1} n^{(k-2) / 2} \exp \left(-n d_{n}\right)$

and

(5.3.3) $\quad \alpha_{n}^{+}\left(\beta, \theta_{1}\right) \geq c_{2} n^{-k / 2} \exp \left\{-n d_{n}-c_{3}(\log n)^{\frac{1}{2}}\right\}$,

where $d_{n}$ is the critical value of the LR test with power $\beta$ at $\theta_{1}(0<\beta<1)$. (5.3.2) is by now a familiar statement, but (5.3.3) is a new inequality. So far we only obtained a lower bound for $\alpha_{n}^{+}$under the assumption that the shortcoming of the LR test is bounded away from zero.

B. We derive an expansion for $d_{n}$. This expansion is used to translate $(5.3 .2)$ and (5.3.3) in terms of $\mathrm{N}^{+}\left(\alpha, \beta, \theta_{1}\right)$ and $\mathrm{N}^{\mathrm{LR}}\left(\alpha, \beta, \theta_{1}\right)$.

$C$. With the aid of $A$ and $B$ the theorem is proved. 
A. To prove (5.3.2) we only have to show that $\alpha_{n}^{L R}\left(\beta, \theta_{1}\right) \geq \exp (-n I)$ for some $0<I<I\left(\theta_{0}\right)$ and sufficiently large $n$. Application of lemma 3.3.3 then immediately yields (5.3.2). Let $\theta_{0} \in \theta_{0}$ be such that $I\left(\theta_{1}, \theta_{0}\right)<I\left(\theta_{0}\right)$. Consider the problem of testing $\mathrm{H}_{0}^{*}: \theta=\theta_{0}$ against $\mathrm{H}_{1}^{*}: \theta=\theta_{1}$. Let $\alpha_{n}^{*}(\beta)$ be the smallest size such that the power of the MP test of $H_{0}^{*}$ against $\mathrm{H}_{1}^{*}$ at $\theta_{1}$ equals $\beta$. Then by Stein's lemma $\lim _{n \rightarrow \infty} \mathrm{n}^{-1} \log \alpha_{n}^{*}(\beta)=-I\left(\theta_{1}, \theta_{0}\right)$ (cf. lemma 6.1 in [4]). Since $\alpha_{n}^{L R}\left(\beta, \theta_{1}\right) \geq \alpha_{n}^{*}(\beta)$ it is plain that there exists a number I satisfying $0<I<I\left(\theta_{0}\right)$ such that $\alpha_{n}^{L R}\left(\beta, \theta_{1}\right) \geq \exp (-n I)$ for sufficiently large $n$.

To prove the required inequality for the MP test is more difficult. We try to find a point $s_{n}$ in $\lambda\left(\theta_{1}\right)$ such that $s_{n}$ has a sufficiently large neighbourhood contained in the critical region of the MP test and such that $\lambda^{-1}\left(s_{n}\right)$ is not too far from $\theta_{0}$. We consider the MP test a little more closely. The critical function of the MP test satisfies

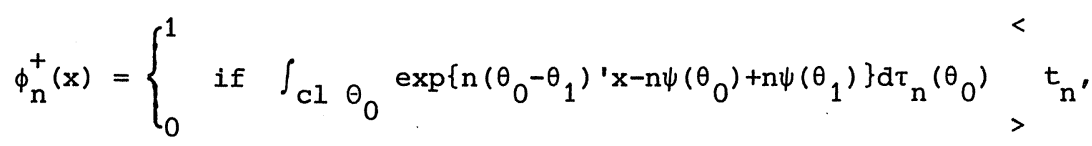

where the distribution $\tau_{\mathrm{n}}$ is least favorable. Denote by

$$
\begin{aligned}
t\left(\theta_{0}, x\right) & =\exp \left\{n\left(\theta_{0}-\theta_{1}\right) \cdot x-n \psi\left(\theta_{0}\right)+n \psi\left(\theta_{1}\right)\right\}, \\
U_{n} & =\left\{x_{i} \int_{c l} \theta_{0} t\left(\theta_{0}, x\right) d \tau_{n}\left(\theta_{0}\right)<t_{n}\right\} \quad \text { and } \\
v_{n} & =\left\{x_{i} \int_{c l} \theta_{0} t\left(\theta_{0}, x\right) d \tau_{n}\left(\theta_{0}\right) \leq t_{n}\right\} .
\end{aligned}
$$

In the course of the proof of theorem 3.3.2 we have shown that both $U_{n}$ and $v_{n}$ are convex sets and that $\operatorname{int}\left(v_{n}-U_{n}\right)=\varnothing$. Let

$$
\begin{aligned}
& \mathrm{H}_{\mathrm{n}}=\left\{\mathrm{x} \in \mathrm{U}_{\mathrm{n}} ;\right. \text { there exists a sphere with radius } \\
& \left.\mathrm{C}_{4} \mathrm{n}^{-1} \text { containing } \mathrm{x} \text { completely contained in } U_{n}\right\} \text {. }
\end{aligned}
$$

Here the constant $c_{4}$ has to be so large that for any sphere $\tilde{B}_{n}$ with centre 0 and radius $\mathrm{c}_{4} \mathrm{n}^{-1}$ there exists a constant $c_{5}$ such that

$$
P_{\tilde{\theta}}\left(\overline{\mathrm{x}}_{\mathrm{n}}-\lambda(\tilde{\theta}) \in \tilde{\mathrm{B}}_{\mathrm{n}}\right) \geq \mathrm{c}_{5} \mathrm{n}^{-\mathrm{k} / 2}
$$

for all $\tilde{\theta}$ in some (fixed) neighbourhood of $\theta_{1}$. This can be derived from the 
special Berry-Esseen theorem (15.57), with $\mathbf{s}=2$, on page 153 in Bhattacharya and Rao's book Normal Approximation and Asymptotic Expansions (1976).

Let $d_{n}$ be the critical value of the LR test with power $\beta$ at $\theta_{1}$. Furthermore let

$$
G_{n}=\left\{x \in \Lambda ; I\left(\lambda^{-1}(x), \theta_{0}\right)>d_{n}\right\}
$$

and denote the complement of $G_{n}$ by $G_{n}^{c}$; finally if $F_{n}$ is any set and $t>0$ define $F_{n}(t)=\left\{x ; \inf \left\{\|x-y\| ; y \in F_{n}\right\}<t\right\}$. Next we show that there exists a constant $c_{6}$ such that

$$
G_{n}^{C}\left(c_{6} n^{-1}\{\log n\}^{\frac{1}{2}}\right) \wedge H_{n} \neq \varnothing
$$

To prove this we first restrict the whole $\Lambda$-space to points near $\lambda\left(\theta_{1}\right)$. Let

$$
Q_{n}=\left\{x ;\left\|\Sigma_{\theta_{1}}^{-\frac{1}{2}}\left(x-\lambda\left(\theta_{1}\right)\right)\right\| \leq 2 n^{-\frac{1}{2}}(\log n)^{\frac{1}{2}}\right\}
$$

Because both the MP test and the LR test have power $\beta$ at $\theta_{1}$ it holds that

$$
P_{\theta_{1}}\left(\overline{\mathrm{X}}_{\mathrm{n}} \in \mathrm{G}_{\mathrm{n}}\right)-P_{\theta_{1}}\left(\overline{\mathrm{x}}_{\mathrm{n}} \in \mathrm{U}_{\mathrm{n}} \wedge \mathrm{Q}_{\mathrm{n}}\right) \leq \mathrm{c}_{7^{n}} \mathrm{n}^{-\frac{1}{2}}
$$

Since $0<\beta<1$ we can take $c_{8}$ so large that

$$
P_{\theta_{1}}\left(\bar{x}_{n} \notin U_{n} \wedge Q_{n}, \bar{x}_{n} \in\left\{U_{n} \wedge Q_{n}\right\}\left(c_{8} n^{-1}\right)\right)>c_{7} n^{-\frac{1}{2}}
$$

This implies that $P_{\theta_{1}}\left(\bar{x}_{n} \in\left\{U_{n} \wedge Q_{n}\right\}\left(c_{8} n^{-1}\right)\right)>P_{\theta_{1}}\left(\bar{x}_{n} \in G_{n}\right)$ and hence there exists a point $y_{n} \in G_{n}^{C} \wedge\left\{U_{n} \wedge Q_{n}\right\}\left(c_{\beta}{ }^{-1}\right)$. Since $\beta>0$ the set $U_{n} \wedge Q_{n}$ contains a sphere $R_{n}$ wj.th radius $c_{9} n^{-\frac{\rho}{2}}$ and centre $r_{n}$. Take $z_{n} \in U_{n} \wedge Q_{n}$ such that $\left\|\mathrm{Y}_{\mathrm{n}}{ }^{-z_{n}}\right\|<\mathrm{c}_{8} \mathrm{n}^{-1}$ and define

$$
s_{n}=z_{n}+c_{4} c_{9}^{-1} n^{-\frac{1}{2}}\left(r_{n}-z_{n}\right) .
$$

Note that the sphere $s_{n}$ with centre $s_{n}$ and radius $c_{4} n^{-1}$ is contained in the cone determined by $R_{n}$ and $z_{n}$.

It is easily seen that $s_{n} \in H_{n}$ for sufficiently large $n$, since $s_{n}$ in $U_{n} \wedge Q_{n}$. Moreover $y_{n} \in G_{n}^{C}$ and

$$
\begin{aligned}
\left\|s_{n}-y_{n}\right\| & \leq\left\|s_{n}-z_{n}\right\|+\left\|z_{n}-y_{n}\right\|<2 \gamma c_{4} c_{9}^{-1} n^{-1}(\log n)^{\frac{1}{2}}+c_{8} n^{-1} \\
& \leq c_{6} n^{-1}(\log n)^{\frac{1}{2}}
\end{aligned}
$$


where $\gamma$ is the largest eigenvalue of $\Sigma_{\theta_{1}}^{\frac{1}{2}}$ and (5.3.5) is proved. We still have to show that $\lambda^{-1}\left(s_{n}\right)$ is not "too far" from $\theta_{0}$. It is easy to see that $y_{n} \in \Lambda$ for sufficiently large $n$; hence $y_{n} \in G_{n}^{c} \wedge \Lambda$ and thus $I\left(\lambda^{-1}\left(y_{n}\right), \theta_{0}\right) \leq d_{n}$. So there exists a point $\theta_{0 n} \in \operatorname{cl} \theta_{0}$ such that $I\left(\lambda^{-1}\left(y_{n}\right), \theta_{0 n}\right) \leq d_{n}$. Consequently

$$
\begin{aligned}
I\left(\lambda^{-1}\left(s_{n}\right), \theta_{0 n}\right) & =I\left(\lambda^{-1}\left(y_{n}\right), \theta_{0 n}\right)+I\left(\lambda^{-1}\left(s_{n}\right), \lambda^{-1}\left(y_{n}\right)\right)+ \\
& +\left(\lambda^{-1}\left(y_{n}\right)-\theta_{0 n}\right) \cdot\left(s_{n}-y_{n}\right) \\
\leq & a_{n}+c_{10} n^{-1}(\log n)^{\frac{1}{2}} .
\end{aligned}
$$

In view of (5.3.4) we obtain the desired lower bound for $\alpha_{n}^{+}\left(\beta, \theta_{1}\right)$ :

$$
\begin{aligned}
& \alpha_{n}^{+}\left(\beta, \theta_{1}\right) \geq P_{\theta_{0 n}}\left(\bar{x}_{n} \in s_{n}\right) \\
& =\int_{S_{n}} \exp \left\{n\left(\theta_{0 n}-\lambda^{-1}\left(s_{n}\right)\right) \cdot x-n \psi\left(\theta_{0 n}\right)+n \psi\left(\lambda^{-1}\left(s_{n}\right)\right)\right\} d \bar{P}^{-n} \lambda^{-1}\left(s_{n}\right) \\
& \geq c_{11} n^{-k / 2} \exp \left\{-n I\left(\lambda^{-1}\left(s_{n}\right), \theta_{0 n}\right)\right\} \\
& \geq c_{11} n^{-k / 2} \exp \left\{-n d_{n}-c_{10}(\log n)^{\frac{1}{2}}\right\},
\end{aligned}
$$

which completes the proof of part $A$.

B. The proof precedes in several steps. We use the following property of $d_{n}$ :

$$
\begin{aligned}
& P_{\theta_{1}}\left(\overline{\mathrm{x}}_{\mathrm{n}} \in \Lambda, I\left(\lambda^{-1}\left(\overline{\mathrm{x}}_{\mathrm{n}}\right), \theta_{0}\right) \geq \mathrm{d}_{\mathrm{n}}\right)+P_{\theta_{1}}\left(\overline{\mathrm{x}}_{\mathrm{n}} \notin \Lambda\right) \geq \beta \geq \\
& \geq P_{\theta_{1}}\left(\overline{\mathrm{x}}_{\mathrm{n}} \in \Lambda, I\left(\lambda^{-1}\left(\overline{\mathrm{x}}_{\mathrm{n}}\right), \theta_{0}\right)>\mathrm{d}_{\mathrm{n}}\right) .
\end{aligned}
$$

For $x \in \Lambda$ and $\theta_{0} \in \theta_{0}$

$$
I\left(\lambda^{-1}(x), \theta_{0}\right)=I\left(\theta_{1}, \theta_{0}\right)+\left(\theta_{1}-\theta_{0}\right) \cdot\left(x-\lambda\left(\theta_{1}\right)\right)+I\left(\lambda^{-1}(x), \theta_{1}\right)
$$

implying

$$
\begin{aligned}
I\left(\lambda^{-1}(x), \theta_{0}\right) & \geq I\left(\theta_{1}, \theta_{0}\right)+\inf _{\theta_{0} \in \theta_{0}}\left(\theta_{1}-\theta_{0}\right) \cdot\left(x-\lambda\left(\theta_{1}\right)\right)+I\left(\lambda^{-1}(x), \theta_{1}\right) \\
& \leq I\left(\theta_{1}, \theta_{0}\right)+\sup _{\theta_{0} \epsilon \theta_{0}}\left(\theta_{1}-\theta_{0}\right) \cdot\left(x-\lambda\left(\theta_{1}\right)\right)+I\left(\lambda^{-1}(x), \theta_{1}\right) .
\end{aligned}
$$


Let $B_{n}(c)$ be the sphere with centre $\lambda\left(\theta_{1}\right)$ and radius $\mathrm{cn}^{-\frac{1}{2}}$. Choose $c_{12}$ so large that

$$
P_{\theta_{1}}\left(\bar{x}_{n} \notin B_{n}\left(C_{12}\right)\right) \leq \min \left(\frac{1}{2} \beta, \frac{1}{2}(1-\beta)\right) .
$$

If $\mathrm{x} \in \mathrm{B}_{\mathrm{n}}\left(\mathrm{c}_{12}\right) \wedge \Lambda$ then

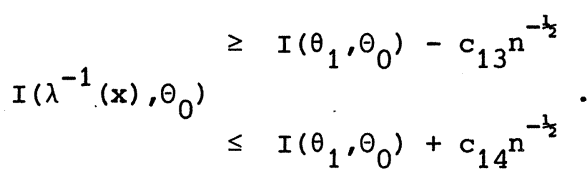

Define $h_{n}$ by

(5.3.8) $\quad d_{n}=I\left(\theta_{1}, \theta_{0}\right)+h_{n} n^{-\frac{1}{2}}$.

The preceeding inequalities imply

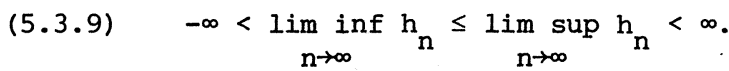

Next we consider two non-decreasing sequences $\left\{n_{j}\right\}$ and $\left\{m_{j}\right\}$ of positive integers tending to infinity, which will later correspond to $\mathrm{N}^{+}\left(\alpha, \beta, \theta_{1}\right)$ and $N^{L R}\left(\alpha, \beta, \theta_{1}\right)$, respectively. Let $\left\{\delta_{j}\right\}$ be a sequence of positive real numbers such that $\mathrm{m}_{j}^{-\frac{1}{2}}\left(\log \mathrm{m}_{j}\right)^{\frac{1}{2}} \leq \delta_{j} \leq 1$. We will prove that if $\delta_{j}$ is "too large" then

$$
\begin{aligned}
& P_{\theta_{1}}\left(\overline{\mathrm{x}}_{\mathrm{m}_{j}} \in \Lambda,\left\{I\left(\lambda^{-1}\left(\overline{\mathrm{x}}_{\mathrm{m}_{j}}\right), \theta_{0}\right)-I\left(\theta_{1}, \theta_{0}\right)\right\} \mathrm{m}_{j}^{\frac{1}{2}} \geq \mathrm{h}_{\mathrm{n}_{j}}+\delta_{j}\right)+ \\
& P_{\theta_{1}}\left(\overline{\mathrm{x}}_{\mathrm{m}_{j}} \notin \Lambda\right)<\beta
\end{aligned}
$$

and

$(5.3 .11)$

$$
P_{\theta_{1}}\left(\bar{x}_{m_{j}} \in \Lambda,\left\{I\left(\lambda^{-1}\left(x_{m_{j}}\right), \theta_{0}\right)-I\left(\theta_{1}, \theta_{0}\right)\right\} m_{j}^{\frac{1}{2}}>h_{n_{j}}-\delta_{j}\right)>\beta
$$

for sufficiently large $j$. In the sequel the meaning of $\delta_{j}$ "too large" will be explained. Since $(5.3 .10)$ and $(5.3 .11)$ require a similar approach we only consider $(5 \cdot 3.10)$.

Without loss of generality assume that the radius $c_{12} m_{j}^{-\frac{3}{2}}$ of $B_{m_{j}}\left(c_{12}\right)$ is so large that for all $j$

(5.3.12) $\inf \left[\left\{I\left(\lambda^{-1}(x), \theta_{0}\right)-I\left(\theta_{1}, \theta_{0}\right)\right\} m_{j}^{\frac{1}{2}} ; x \in B_{m_{j}}\left(\frac{1}{2} C_{12}\right)\right]<\inf h_{n}$ 
(cf. (5.3.9)). Since for all $\mathrm{x}$ in a sufficiently small neighbourhood of $\lambda\left(\theta_{1}\right)$

(5.3.13) I I ( $\left.\lambda^{-1}(x), \theta_{0}\right)=\inf _{\theta_{0} \in \Theta_{0}}\left\{I\left(\theta_{1}, \theta_{0}\right)+\left(\theta_{1}-\theta_{0}\right) \cdot\left(x-\lambda\left(\theta_{1}\right)\right)\right\}+I\left(\lambda^{-1}(x), \theta_{1}\right)$

and therefore

$$
\begin{aligned}
I\left(\lambda^{-1}(x), \theta_{0}\right) & \geq \inf _{\theta_{0} \epsilon \theta_{0}}\left\{I\left(\theta_{1}, \theta_{0}\right)+\left(\theta_{1}-\theta_{0}\right) \cdot\left(x-\lambda\left(\theta_{1}\right)\right)\right\} \\
& \leq \inf _{\theta_{0} \epsilon \theta_{0}}\left\{I\left(\theta_{1}, \theta_{0}\right)+\left(\theta_{1}-\theta_{0}\right) \cdot\left(x-\lambda\left(\theta_{1}\right)\right)\right\}+c_{15} m_{j}^{-1},
\end{aligned}
$$

it follows that

$(5.3 .14)$

$$
\begin{aligned}
& P_{\theta_{1}}\left(\bar{x}_{m_{j}} \in B_{m_{j}}\left(c_{12}\right),\left\{I\left(\lambda^{-1}\left(\bar{x}_{m_{j}}\right), \theta_{0}\right)-I\left(\theta_{1}, \theta_{0}\right)\right\} m_{j}^{\frac{1}{2}} \in\left[h_{n_{j}}+\frac{1}{2} \delta_{j}, h_{n_{j}}+\delta_{j}\right)\right) \\
& \geq P_{\theta_{1}}\left(\bar{x}_{m_{j}} \in B_{m_{j}}\left(c_{12}\right),\left[i n \theta _ { 0 } \in \theta _ { 0 } \left\{I\left(\theta_{1}, \theta_{0}\right)+\left(\theta_{1}-\theta_{0}\right) \cdot\left(\bar{x}_{m_{j}}-\lambda\left(\theta_{1}\right)\right\}-\right.\right.\right. \\
& \left.\left.I\left(\theta_{1}, \theta_{0}\right)\right] \mathrm{m}_{j}^{\frac{1}{2}} \in\left[\mathrm{h}_{n_{j}}+\frac{1}{2} \delta_{j}, h_{n_{j}}+\delta_{j}-c_{15} m_{j}^{-\frac{1}{2}}\right)\right) \\
& \geq \operatorname{Pr}\left(\|Y\| \leq C_{12},\left[\inf _{\theta_{0}} \epsilon \Theta_{0}\left\{I\left(\theta_{1}, \theta_{0}\right)+\left(\theta_{1}-\theta_{0}\right) ' Y m_{j}^{-\frac{1}{2}}\right\}-I\left(\theta_{1}, \theta_{0}\right)\right] m_{j}^{\frac{3}{2}}\right. \\
& \left.\epsilon\left[h_{n_{j}}+\frac{1}{2} \delta_{j}, h_{n_{j}}+\delta_{j}-c_{15^{m}} m^{-\frac{k}{2}}\right)\right)-c_{16^{-}} m_{j}^{-\frac{1}{2}} \text {, }
\end{aligned}
$$

where $\mathrm{Y}$ is a normally $\mathrm{N}\left(0, \Sigma_{\theta_{1}}\right)$ distributed random $\mathrm{k}$-vector and where in the last inequality the convexity of the sets

$$
\begin{aligned}
& \left\{x_{i}\left[\inf _{\theta_{0}} \in \theta_{0}\left\{I\left(\theta_{1}, \theta_{0}\right)+\left(\theta_{1}-\theta_{0}\right) \cdot\left(x-\lambda\left(\theta_{1}\right)\right)\right\}-I\left(\theta_{1}, \theta_{0}\right)\right] m_{j}^{\frac{1}{2}} \geq\right. \\
& \left.\geq h_{n_{j}}+\delta_{j}-n\right\}
\end{aligned}
$$

( $n=0$ or $c_{15} \mathrm{~m}^{-\frac{1}{2}}$ ) is used (cf. SAZONOV (1968)).

For all $j \geq j_{0}$ it holds that for every $t$ satisfying $\|t\| \leq \frac{3}{4} c_{12}$ and

$$
\left[\inf _{\theta_{0} \in \theta_{0}}\left\{I\left(\theta_{1}, \theta_{0}\right)+\left(\theta_{1}-\theta_{0}\right) \cdot \mathrm{tm}_{j}^{-\frac{1}{2}}\right\}-I\left(\theta_{1}, \theta_{0}\right)\right] \mathrm{m}_{j}^{\frac{1}{2}}=h_{n_{j}}+\frac{3}{4} \delta_{j},
$$

there exists a positive constant $c_{17}$ such that the sphere

$$
\text { (5.3.15) } \quad\left\{y ;\left\|_{y}-t\right\| \leq c_{17} \delta_{j}\right\}
$$

is contained in the set 
$(5.3 .16)$

$$
\begin{aligned}
& \left\{y ;\|y\| \leq c_{12},\left[\inf _{\theta_{0}} \in \theta_{0}\left\{I\left(\theta_{1}, \theta_{0}\right)+\left(\theta_{1}-\theta_{0}\right) ' y m_{j}^{-\frac{1}{2}}\right\}-I\left(\theta_{1}, \theta_{0}\right)\right] m_{j}^{\frac{1}{2}}\right. \\
& \left.\epsilon\left[h_{n_{j}}+\frac{1}{2} \delta_{j}, h_{n_{j}}+\delta_{j}-c_{15} m_{j}^{-\frac{1}{2}}\right)\right\} .
\end{aligned}
$$

From now on let $j \geq j_{0}$. Without loss of generality we may assume that

$$
P_{\theta_{1}}\left(\bar{x}_{m_{j}} \in \Lambda,\left\{I\left(\lambda^{-1}\left(\bar{x}_{m_{j}}\right), \theta_{0}\right)-I\left(\theta_{1}, \theta_{0}\right)\right\} m_{j}^{\frac{1}{2}} \geq h_{n_{j}}+\delta_{j}\right) \geq \frac{3}{4} \beta
$$

since otherwise $(5.3 .10)$ is trivially satisfied for large $j$. Hence

$$
\begin{aligned}
& P_{\theta_{1}}\left(\bar{x}_{m_{j}} \in B_{m_{j}}\left(\frac{1}{2} c_{12}\right),\left[i n \theta_{0} \in \theta_{0}\left\{I\left(\theta_{1}, \theta_{0}\right)+\left(\theta_{1}-\theta_{0}\right) \cdot\left(\bar{x}_{m_{j}}-\lambda\left(\theta_{1}\right)\right)\right\}-\right.\right. \\
& \left.\left.I\left(\theta_{1}, \theta_{0}\right)\right] m_{j}^{\frac{1}{2}} \geq h_{n_{j}}+\delta_{j}-c_{15} m_{j}^{-\frac{1}{2}}\right) \geq \frac{1}{4} \beta .
\end{aligned}
$$

In view of this inequality there is a sphere with radius $c_{18} \mathrm{~m}_{j}^{-\frac{1}{2}}$ in $B_{m_{j}}\left(\frac{1}{2} c_{12}\right)$ such that the function $\left[\inf _{\theta_{0} \in \Theta_{\rho}}\left\{I\left(\theta_{1}, \theta_{0}\right)+\left(\theta_{1}-\theta_{0}\right) \cdot\left(x-\lambda\left(\theta_{1}\right)\right)\right\}-I\left(\theta_{1}, \theta_{0}^{j}\right)\right] \mathrm{m}_{j}^{\frac{1}{2}}$ is larger than $h_{n_{j}}+\delta_{j}-c_{15} m_{j}^{-\frac{1}{2}}$ on that sphere and by (5.3.12) there is $a$ point in $B_{m_{j}}\left(\frac{1}{2} C_{12}\right)$ such that the same function is smaller than $h_{n_{j}}$. It follows that there exists a point $t_{j}$ on the surface

$$
\begin{aligned}
A_{j} & =\left\{t ;\left[\inf _{\theta_{0}} \epsilon \theta_{0}\left\{I\left(\theta_{1}, \theta_{0}\right)+\left(\theta_{1}-\theta_{0}\right) \cdot t m_{j}^{-\frac{1}{2}}\right\}-I\left(\theta_{1}, \theta_{0}\right)\right] m_{j}^{\frac{1}{2}}\right. \\
& \left.=h_{n_{j}}+\frac{3}{4} \delta_{j}\right\}
\end{aligned}
$$

satisfying $\left\|t_{j}\right\| \leq \frac{1}{2} c_{12}$. Let $c_{19}=\min \left(c_{18^{\prime}}{ }^{\frac{1}{4} c_{12}}\right)$. Then the sphere

$$
A_{j}^{*}=\left\{y ;\left\|_{y-t}\right\| \leq c_{19}\right\}
$$

is contained in

$$
\left\{y ;\|y\| \leq \frac{3}{4} c_{12}\right\}
$$

The set $A_{j}$ is not contained in $A_{j}^{*}$ for all $j \geq j_{1}$, since $c_{19} \leq c_{18}$ and, for all sufficiently large $j$; the set

$$
\left\{y ;\left[\inf _{\theta_{0} \in \Theta_{0}}\left\{I\left(\theta_{1}, \theta_{0}\right)+\left(\theta_{1}-\theta_{0}\right) ' y_{j}^{-\frac{1}{2}}\right\}-I\left(\theta_{1}, \theta_{0}\right)\right] m_{j}^{\frac{1}{2}} \geq h_{n_{j}}+\frac{3}{4} \delta_{j}\right\}
$$

contains the set

$$
\left\{y ;\|y\| \leq \frac{1}{2} C_{12} ;\left[i n \theta_{\theta_{0}} \in \theta_{0}\left\{I\left(\theta_{1}, \theta_{0}\right)+\left(\theta_{1}-\theta_{0}\right) \cdot y m_{j}^{-\frac{1}{2}}\right\}-I\left(\theta_{1}, \theta_{0}\right)\right] m_{j}^{\frac{1}{2}} \geq\right.
$$




$$
\left.\geq h_{n_{j}}+\delta_{j}-c_{15^{m}} m_{j}^{-\frac{1}{2}}\right\} .
$$

From now on let $j \geq j_{1}$. It follows that the area of

$$
\begin{aligned}
& \left\{t ;\|t\| \leq \frac{3}{4} c_{12},\left[i n \theta_{0} \in \Theta_{0}\left\{I\left(\theta_{1}, \theta_{0}\right)+\left(\theta_{1}-\theta_{0}\right) \cdot \operatorname{tm}_{j}^{-\frac{3}{2}}\right\}-I\left(\theta_{1}, \theta_{0}\right)\right] m_{j}^{\frac{1}{2}}=\right. \\
& \left.h_{n_{j}}+\frac{3}{4} \delta_{j}\right\}
\end{aligned}
$$

is at least equal to some positive constant $c_{20}$. Therefore

$(5.3 .17)$

$$
\begin{aligned}
& P_{\theta_{1}}\left(\bar{x}_{m_{j}} \in B_{m_{j}}\left(c_{12}\right) ;\left\{I\left(\lambda^{-1}\left(\bar{x}_{m_{j}}\right), \theta_{0}\right)-I\left(\theta_{1}, \theta_{0}\right)\right\} m_{j}^{\frac{1}{2}} \epsilon\right. \\
& \left.\left[h_{n_{j}}+\frac{1}{2} \delta \delta_{j}, h_{n_{j}}+\delta_{j}\right)\right) \geq c_{21} \delta_{j}-c_{16} m_{j}^{-\frac{1}{2}},
\end{aligned}
$$

cf. $(5.3 .14),(5.3 .15)$ and $(5.3 .16)$.

Let

$(5.3 .18)$

$$
Q_{j}^{*}=\left\{x ;\left\|x-\lambda\left(\theta_{1}\right)\right\| \leq \tau_{j} m_{j}^{-\frac{1}{2}}\right\}
$$

where the sequence $\left\{\tau_{j}\right\}$ will be specified later. Now the following inequality holds for sufficiently large $j$

$(5.3 .19)$

$$
\begin{aligned}
& P_{\theta_{1}}\left(\overline{\mathrm{x}}_{\mathrm{m}_{j}} \in \Lambda,\left\{I\left(\lambda^{-1}\left(\overline{\mathrm{x}}_{\mathrm{m}_{j}}\right), \theta_{0}\right)-I\left(\theta_{1}, \theta_{0}\right)\right\} \mathrm{m}_{j}^{\frac{1}{2}} \geq \mathrm{h}_{\mathrm{n}_{j}}+\delta_{j}\right) \\
& +P_{\theta_{1}}\left(\overline{\mathrm{x}}_{\mathrm{m}_{j}} \notin \Lambda\right) \\
& \leq P_{\theta_{1}}\left(\overline{\mathrm{x}}_{\mathrm{m}_{j}} \in Q_{j}^{*},\left\{I\left(\lambda^{-1}\left(\overline{\mathrm{x}}_{\mathrm{m}_{j}}\right), \theta_{0}\right)-I\left(\theta_{1}, \theta_{0}\right)\right\} \mathrm{m}_{j}^{\frac{1}{2}} \geq \mathrm{h}_{\mathrm{n}_{j}}+\frac{1}{2} \delta_{j}\right) \\
& -\mathrm{c}_{22} \delta_{j}+P_{\theta_{1}}\left(\overline{\mathrm{x}}_{\mathrm{m}_{j}} \notin \mathrm{Q}_{j}^{*}\right) .
\end{aligned}
$$

In view of $(5.3 .13)$ the first term in the righthand member of (5.3.19) is at most equal to

$$
\begin{aligned}
& P_{\theta_{1}}\left(\bar{x}_{m_{j}} \in Q_{j}^{*},{ }^{\text {inf }} \theta_{0} \in \theta_{0}\left\{I\left(\theta_{1}, \theta_{0}\right)-I\left(\theta_{1}, \theta_{0}\right)+\right.\right. \\
& \left.\left.\left(\theta_{1}-\theta_{0}\right)^{\prime}\left(\bar{x}_{m_{j}}-\lambda\left(\theta_{1}\right)\right) m_{j}^{\frac{1}{2}} n_{j}^{-\frac{1}{2}}\right)_{j}^{\frac{1}{2}} \geq h_{n_{j}}+\frac{1}{2} \delta_{j}-c_{23}\left|1-m_{j}^{\frac{1}{2}} n_{j}^{-\frac{1}{2}}\right| \tau_{j}-c_{24} \tau_{j}^{2} m_{j}^{-\frac{1}{2}}\right) \\
& \leq P_{\theta_{1}}\left(\| \overline { x } _ { n _ { j } } - \lambda ( \theta _ { 1 } ) \| \leq \tau _ { j } n _ { j } ^ { - \frac { 1 } { 2 } } , \text { inf } { } _ { \theta _ { 0 } \in \theta _ { 0 } } \left\{I\left(\theta_{1}, \theta_{0}\right)-I\left(\theta_{1}, \theta_{0}\right)+\right.\right.
\end{aligned}
$$




$$
\begin{aligned}
& \left.\left.\left(\theta_{1}-\theta_{0}\right) \cdot\left(\bar{x}_{n_{j}}-\lambda\left(\theta_{1}\right)\right)\right\} m_{j}^{\frac{1}{2}} \geq h_{n_{j}}+\frac{1}{2} \delta_{j}-c_{23}\left|1-m_{j}^{\frac{3}{2}} n_{j}^{-\frac{1}{2}}\right| \tau_{j}-c_{24} \tau_{j}^{2} m_{j}^{-\frac{1}{2}}\right) \\
& +c_{25}\left(m_{j}^{-\frac{1}{2}}+n_{j}^{-\frac{1}{2}}\right),
\end{aligned}
$$

where we applied the multivariate "Berry-Esseen" theorem for convex sets twice (cf. SAZONOV (1968)). In view of (5.3.13) the last probability is at most equal to

$$
\begin{aligned}
& P_{\theta_{1}}\left(\left\|\bar{x}_{n_{j}}-\lambda\left(\theta_{1}\right)\right\| \leq \tau_{j} n_{j}^{-\frac{1}{2}},\left\{I\left(\lambda^{-1}\left(\bar{x}_{n_{j}}\right), \theta_{0}\right)-I\left(\theta_{1}, \theta_{0}\right)\right\} n_{j}^{\frac{1}{2}} \geq\right. \\
& \left.\left\{h_{n_{j}}+\frac{1}{2} \delta_{j}-c_{23}\left|1-m_{j}^{\frac{1}{2}} n_{j}^{-\frac{1}{2}}\right| \tau_{j}-c_{24} \tau_{j}^{2} m_{j}^{-\frac{1}{2}}\right\}_{j}^{\frac{1}{2}} m_{j}^{-\frac{1}{2}}\right) .
\end{aligned}
$$

We consider two cases:

(i) $m_{j}^{-1} n_{j} \rightarrow 1$; choose $\delta_{j}=n$, where $n$ is an arbitrary number in $(0,1)$ and choose $\tau_{j}=c_{26}$, where $c_{26}$ is so large that

$$
P_{\theta_{1}}\left(\bar{x}_{m_{j}} \notin Q_{j}^{*}\right) \leq \frac{1}{2} c_{22} n
$$

(ii) $\left(m_{j}-n_{j}\right) m_{j}^{-\frac{1}{2}} \rightarrow 0$; choose $\tau_{j}=c_{27}\left(\log m_{j}\right)^{\frac{1}{2}}$ where $c_{27}$ is so large that

$$
P_{\theta_{1}}\left(\bar{x}_{m_{j}} \notin Q_{j}^{*}\right) \leq m_{j}^{-\frac{1}{2}},
$$

and choose $\delta_{j}=\left(2+2 c_{24} c_{27}^{2}\right) m_{j}^{-\frac{1}{2}} \log m_{j}$.

Now in both cases

$$
\left\{h_{n_{j}}+\frac{1}{2} \delta_{j}-c_{23}\left|1-m_{j}^{\frac{1}{2}} n_{j}^{-\frac{1}{2}}\right| \tau_{j}-c_{24} \tau_{j}^{2} m_{j}^{-\frac{1}{2}}\right\} n_{j}^{\frac{1}{2}} m_{j}^{-\frac{1}{2}}>h_{n_{j}}
$$

for sufficiently large $j$ and hence the probability $(5.3 .21)$ is at most equal to $\beta$ (cf. (5.3.6) and (5.3.8)). In combination with (5.3.19) and (5.3.20) this implies that

$$
\begin{aligned}
& P_{\theta_{1}}\left(\bar{x}_{m_{j}} \in \Lambda,\left\{I\left(\lambda^{-1}\left(\bar{x}_{m_{j}}\right), \theta_{0}\right)-I\left(\theta_{1}, \theta_{0}\right)\right\} m_{j}^{\frac{1}{2}} \geq h_{n_{j}}+\delta_{j}\right) \\
& +P_{\theta_{1}}\left(\bar{x}_{m_{j}} \notin \Lambda\right) \leq \beta+c_{25}\left(m_{j}^{-\frac{1}{2}}+n_{j}^{-\frac{1}{2}}\right)-c_{22} \delta_{j}+P_{\theta_{1}}\left(\bar{x}_{m_{j}} \notin Q_{j}^{*}\right) .
\end{aligned}
$$

In both cases the righthand member of (5.3.22) is less than $\beta$ for sufficiently large $j$. This completes the proof of (5.3.10).

$$
\text { Therefore, if } m_{j}^{-1} n_{j} \rightarrow 1 \text { it follows that } h_{m_{j}}<h_{j}+n \text { for sufficiently }
$$


large $j$, where $n$ is an arbitrary number in $(0,1)$. Moreover, if $\left(m_{j}-n_{j}\right) m_{j}^{-\frac{1}{2}} \rightarrow 0$, then $h_{m_{j}}<h_{n_{j}}+\left(2+2 c_{24} c_{27}^{2}\right) m_{j}^{-\frac{1}{2}} \log m_{j}$. Reverse inequalities of the type $h_{m}>h_{n_{1}}-\ldots$ follow similarly from (5.3.11).

Summarizing the results of part $B$ we have found thus far

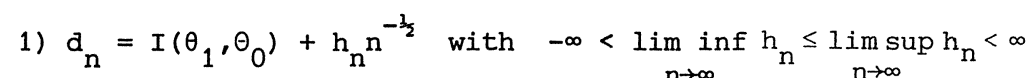

2) if $m_{j}^{-1} n_{j} \rightarrow 1$ then $a_{m_{j}}-d_{n_{j}}=o\left(m_{j}^{-\frac{1}{2}}\right)$ as $j \rightarrow \infty$

$(5.3 .25)$

3) if $\left(m_{j}-n_{j}\right) m_{j}^{-\frac{1}{2}} \rightarrow 0$ then $a_{m_{j}}-d_{n_{j}}=0\left(m_{j}^{-1} \log m_{j}\right)$ as $j \rightarrow \infty$.

C. We write $M$ and $N$ in lieu of $N^{+}\left(\alpha, \beta, \theta_{1}\right)$ and $N^{L R}\left(\alpha, \beta, \theta_{1}\right)-1$, respectively. By $(5.3 .2),(5.3 .3)$ and $(5.1 .3)$

$$
\mathrm{C}_{2} \mathrm{M}^{-\mathrm{k} / 2} \exp \left\{-\mathrm{Md} \cdot \mathrm{M}^{\left.-\mathrm{C}_{3}(\log M)^{\frac{1}{2}}\right\}} \leq \mathrm{C}_{1} \mathrm{~N}^{(\mathrm{k}-2) / 2} \exp (-\mathrm{Nd} \mathrm{N})\right.
$$

or $($ cf. $(5.3 .23))$

$$
\begin{aligned}
& \mathrm{c}_{2} M^{-\mathrm{k} / 2} \exp \left\{-M I\left(\theta_{1}, \theta_{0}\right)-\mathrm{h}_{M} M^{\frac{1}{2}}-\mathrm{c}_{3}(\log M)^{\frac{1}{2}}\right\} \leq \\
& \leq \mathrm{c}_{1} \mathrm{~N}^{(\mathrm{k}-2) / 2} \exp \left\{-\mathrm{NI}\left(\theta_{1}, \theta_{0}\right)-\mathrm{h}_{\left.\mathrm{N} \mathrm{N}^{\frac{1}{2}}\right\}}\right.
\end{aligned}
$$

which implies that $\mathrm{MN}^{-1} \rightarrow 1$. Hence by $(5.3 .24) \mathrm{d}_{\mathrm{N}}-\mathrm{d}_{\mathrm{M}}=O\left(\mathrm{~N}^{-\frac{1}{2}}\right)$. Let $\mathrm{f}_{\mathrm{N}}=$ $(N-M) N^{-\frac{1}{2}}$ and $d_{M}=d_{N}+\varepsilon_{N} N^{-\frac{1}{2}}$ with $\varepsilon_{N} \rightarrow 0$. In view of $(5.3 .26)$ we obtain

$$
\exp \left\{f_{N} N^{\frac{1}{2}} d_{N}-\varepsilon_{N} N^{\frac{1}{2}}+f_{N} \varepsilon_{N}-c_{3}(\log N)^{\frac{1}{2}}\right\} \leq c_{28} N^{k-1} .
$$

This implies that $f_{N} \rightarrow 0$ and therefore by $(5.3 .25) d_{N}-d_{M}=O\left(N^{-1} \log N\right)$. Hence there exists a constant $c_{29}$ such that $d_{M} \leq d_{N}+c_{29} N^{-1} \log N$. Let $g_{N}=(N-M)(\log N)^{-1}$; then

$$
\begin{aligned}
& \exp \left\{-c_{29} \log N+g_{N} d_{N} \log N+c_{29} g_{N} N^{-1}(\log N)^{2}-c_{3}(\log N)^{\frac{1}{2}}\right\} \leq \\
& \leq c_{28}{ }^{k-1}
\end{aligned}
$$

and hence

$$
g_{N} \leq\left\{I\left(\theta_{1}, \theta_{0}\right)\right\}^{-1}\left\{c_{29}+k-1+\varepsilon\right\}
$$

for sufficiently large $N$, where $\varepsilon$ is an arbitrary positive number. 
This completes the proof.

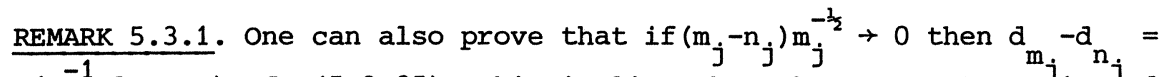
$o\left(m_{j}^{-1} \log m_{j}\right)$, cf. (5.3.25). This implies that the constant $c_{29}{ }_{j}{ }_{i s}{ }^{n_{j}}$ tedundant in the upper bound for $g_{\mathrm{N}}$. For technical reasons we omit the proof of this refinement.

As in chapter III we can also consider the case of an alternative hypothesis set contained in a compact subset of int $\theta$.

THEOREM 5.3.3. Let $\theta_{1}$ be a subset of a compact subset of int $\theta$ and let $\theta_{0}=\theta-\theta_{1}$. Then for every $\theta \in \theta_{1}$ with $I\left(\theta, \theta_{0}\right)>0$ the LR test is deficient in the sense of Bahadur at $\theta$ of order $O\left(\log \mathrm{N}^{+}(\alpha, \beta, \theta)\right)$ as $\alpha \rightarrow 0$.

The proof is based on the fact that only that part of $\theta_{0}$ plays a part which is near $\theta_{1}$ and hence all relevant arguments are concerned with a compact subset of int $\theta$. Since we have shown this in detail in section 3.4, we here omit the proof.

Let $\theta_{0}$ be a Borel set in $\mathbb{R}^{\mathrm{k}}$. Consider the testing problem $\mathrm{H}_{0}: \theta \in \theta_{0}$ against $\mathrm{H}_{1}$ : $\theta \notin \theta_{0}$. We make some assumptions similar to those mentioned in section 3.5 .

ASSUMPTION A1. For all $\mathrm{n}$ the LR test satisfies

$$
\frac{\sup _{\theta_{0} \epsilon \Theta_{0} \wedge \mathrm{K}} \mathrm{E}_{\theta_{0}}{ }_{\mathrm{n}}^{\mathrm{LR}}\left(\overline{\mathrm{x}}_{\mathrm{n}}\right)}{\sup _{\theta \epsilon \Theta_{0}}{ }^{\mathrm{E}}{ }_{\theta} \phi_{\mathrm{n}}^{\mathrm{LR}}\left(\overline{\mathrm{x}}_{\mathrm{n}}\right)} \geq \varepsilon
$$

for some compact subset $\mathrm{K}$ of int $\theta$ and some $\varepsilon>0$.

ASSUMPTION A2. $0<I\left(\theta_{1}, \theta_{0}\right)<I\left(\theta_{0} \wedge \mathrm{K}\right)$, where $\mathrm{K}$ is defined in assumption $\mathrm{A} 1$. THEOREM 5.3.4. Let assumption A1 be fulfilled. The LR test of $\mathrm{H}_{0}: \theta \in \cup_{0}$ against $\mathrm{H}_{1}: \theta \notin \theta_{0}$ is deficient in the sense of Bahadur at $\theta_{1}$ of order $O\left(\log \mathrm{N}^{+}\left(\alpha, \beta, \theta_{1}\right)\right)$ as $\alpha \rightarrow 0$ for those points $\theta_{1} \in$ int $\theta_{1}$ for which assumption A2 is satisfied.

PROOF. Assumption A2 implies that $\alpha_{n}^{L R}\left(\beta, \theta_{1}\right) \geq \exp (-n I)$ for some $0<I<$ $I\left(\theta_{0} \wedge \mathrm{K}\right)$. Arguments are similar to those in the first paragraph of part $A$ of the proof of theorem 5.3.2. By assumption A1 and lemma 3.3.3 it then follows that 


$$
\alpha_{n}^{L R}\left(\beta, \theta_{1}\right) \leq c_{1} n^{(k-2) / 2} \exp \left(-n d_{n}\right)
$$

for some positive constant $c_{1}$.

The remainder of the proof follows the lines of the proof of theorem 5.3.2. Since by lemma 4.1 .2 there exists a positive constant $c_{2}$ such that

$$
I\left(\lambda^{-1}(x), \theta_{0}\right)=\inf _{\substack{\left\|\theta_{0}-\theta_{1}\right\| \leq c \\ \theta_{0} \in \Theta_{0}}} I\left(\lambda^{-1}(x), \theta_{0}\right)
$$

for all $\mathrm{x}$ in a (sufficiently small) neighbourhood of $\lambda\left(\theta_{1}\right)$, the compactness of $\theta_{0}$ is not really needed in the remainder of the proof.

As an application of this theorem we consider the t-test. Let $\left\{x_{n}\right\}$ be a sequence of i.i.d. random variables with a normal $\mathrm{N}\left(\mu, \sigma^{2}\right)$ distribution. Consider the testing problem $\mathrm{H}_{0}: \mu=\mu_{0}$ against $\mathrm{H}_{1}: \mu \neq \mu_{0}$, where $\mu_{0}$ is a given constant $\left(-\infty<\mu_{0}<\infty\right)$. The LR test (i.e. the two-sided t-test) is similar; hence assumption $A 1$ is fulfilled for every compact set $\mathrm{K}$. Moreover, assumption $\mathrm{A} 2$ is also satisfied for all points $\left(\mu, \sigma^{2}\right)$ with $\mu \neq \mu_{0}$ (cf. section 3.5). As a consequence of theorem 5.3 .4 we therefore obtain

COROLLARY 5.3.5. The t-test is deficient in the sense of Bahadur at $\left(\mu, \sigma^{2}\right)$ of order $O\left(\log \mathrm{N}^{+}\left(\alpha, \beta,\left(\mu, \sigma^{2}\right)\right)\right)$ as $\alpha \rightarrow 0$ for all points $\left(\mu, \sigma^{2}\right)$ with $\mu \neq \mu_{0}$.

As two further examples we consider the multivariate normal distribution with known covariance matrix and the multinomial distribution (cf. section 3.6 and 3.7 ).

COROLLARY 5.3.6. Let $\mathrm{x}_{1}, \mathrm{x}_{2}, \ldots$ be i.i.d. random $\mathrm{k}$-dimensional vectors normally distributed with unknown expectation $\mu$ and known covariance matrix. Consider the testing problem $\mathrm{H}_{0}: \mu \in \mathrm{M}_{0}$ against $\mathrm{H}_{1}: \mu \notin \mathrm{M}_{0}$, where $\mathrm{M}_{0}$ is an arbitrary subset of $\mathbb{R}^{\mathrm{k}}$. Then the LR test is deficient in the sense of Bahadur at $\mu_{1}$ of order $O\left(\log \mathrm{N}^{+}\left(\alpha, \beta, \mu_{1}\right)\right)$ as $\alpha \rightarrow 0$ for all points $\mu_{1} \epsilon$ $\operatorname{int}\left(\mathbb{R}^{\mathrm{k}}-\mathrm{M}_{0}\right)$.

PROOF. Let $\mu_{1} \epsilon \operatorname{int}\left(\mathbb{R}^{k}-M_{0}\right)$. Although assumption $A 1$ is not necessarily satisfied, the inequality

$$
\alpha_{n}^{L R}\left(\beta, \mu_{1}\right) \leq c n^{(k-2) / 2} \exp \left(-n d_{n}\right)
$$


for some positive constant $c$ follows from (3.6.1). Since this is the only part of the proof requiring the assumptions $A 1$ and $A 2$ (once $\mu_{1} \in \operatorname{int}\left(\mathbb{R}^{\mathrm{k}}-\mathrm{M}_{0}\right.$ ) is assumed) in theorem 5.3.4, this theorem yields the desired result. $\square$

COROLLARY 5.3.7. Let $\mathrm{Y}_{\mathrm{n}}$ be a random vector having a $\mathrm{k}$-dimensional multinomial distribution with parameters $\mathrm{n}$ and $\mathrm{p}=\left(\mathrm{p}^{(1)}, \ldots, \mathrm{p}^{(\mathrm{k})}\right), \mathrm{n}=1,2, \ldots$. Consider the testing problem $\mathrm{H}_{0}: \mathrm{p} \in \Pi_{0}$ against $\mathrm{H}_{1}: \mathrm{p} \in \Pi_{1}=\Pi-\Pi_{0}$, where $I_{0}$ is a subset of $\Pi$ with the property $p \in\left\{p \in \Pi_{i} ; p^{(j)}=0\right.$ for some $\left.j\right\}$ implying $\mathrm{p} \in \mathrm{cl}$ (int $\Pi_{i}$ ), $i=0,1$. Then the LR test is deficient in the sense of Bahadur at $p$ of order $O\left(\log N^{+}(\alpha, \beta, p)\right)$ as $\alpha \rightarrow 0$ for all points $p \in$ int $\Pi_{1}$.

PROOF. The proof is similar to that of the previous corollary; if $p \in$ int $\Pi_{1}$ and the condition on $\Pi_{0}$ is fulfilled the inequality

$$
\alpha_{n}^{L R}(\beta, p) \leq c n^{(k-2) / 2} \exp \left(-n d_{n}\right)
$$

for some positive constant $c$ follows from (3.7.2). $\square$ 



\section{REFERENCES}

[1] BAHADUR, R.R.[1960], Stochastic comparison of tests, Ann. Math. Statist. $31,276-295$.

[2] BAHADUR, R.R.[1965], An optimal property of the likelihood ratio statistic, Proc. Fifth Berkeley Symp. Math. Statist. Prob. 1, 13-26.

[3] BAHADUR, R.R. and M. RAGHAVACHARI [1970], Some asymptotic properties of likelihood ratios on general sample spaces, Proc. Sixth Berkeley Symp. Math. Statist. Prob. 1, 129-152.

[4] BAHADUR, R.R.[1971], Some limit theorems in statistics, SIAM, Philadelphia.

[5] BERK, R.H.[1972], Consistency and asymptotic normality of MLE's for exponential models, Ann. Math. Statist. 43, 193-204.

[6] BOROVKOV, A.A.[1975], Asymptotically optimal tests for compound hypotheses, Theor. Probability Appl. 20, 447-469.

[7] BROWN, L.D.[1971], Non-local asymptotic optimality of appropriate likelihood ratio tests, Ann. Math. Statist. 42, 1206-1240.

[8] EFRON, B. and D. TRUAX [1968], Large deviations theory in exponential families, Ann. Math. Statist. 39, 1402-1424.

[9] HERR, D.G.[1967], Asymptotically optimal tests for multivariate normal distributions, Ann. Math. Statist. 38, 1829-1844.

[10] HOEFFDING, W.[1965a], Asymptotically optimal tests for multinomial distributions, Ann. Math. Statist. 36, 369-401.

[11] HOEFFDING, w.[1965b], On probabilities of large deviations, Proc. Fifth Berkeley Symp. Math. Stat. Prob. 1, 203-219.

[12] KOLMOGOROV, A.[1956], Two uniform limit theorems for sums of independent random variables, Theor. Probability Appl. 1, 384-394.

[13] KOLMOGOROV, A.[1958], Sur les propriétés des fonctions de concentration de M.P. Lévy, Ann. Inst. Henri Poincaré 16, 27-34.

[14] KRAFFT, O. and H. WITTING [1967], Optimale Tests und ungünstigste Verteilungen, $\mathrm{z}$. Wahrscheinlichkeitstheorie verw. Geb. ㄱ, 289-302.

[15] LEHMANN, E.L.[1959], Testing statistical hypotheses, Wiley, New York. 
[16] NEYMAN, J. and E.S. PEARSON [1928], On the use and interpretation of certain test criteria for purposes of statistical inference, Biometrika, 20A, 175-240 and 263-294.

[17] NEYMAN, J. and E.S. PEARSON [1933], On the problem of the most efficient tests of statistical hypotheses, Trans. Roy. Soc. London A 231, 289-337.

[18] OOSTERHOFF, J. and W.R. VAN ZWET [1970], The likelihood ratio test for the multinomial distribution, Proc. Sixth Berkeley Symp. Math. Stat. Prob. 1, 31-50.

[19] RAGHAVACHARI, M.[1970], On a theorem of Bahadur on the rate of convergence of test statistics, Ann. Math. Statist. 41, 1695-1699.

[20] SAZONOV, V.V.[1968], on the multi-dimensional central limit theorem, Sankhya, $\underline{A 30}, 181-204$.

[21] WALD, A.[1943], Tests of statistical hypotheses concerning several parameters when the number of observations is large, Trans. Amer. Math. Soc. 54, 426-482.

[22] WILKS, S.S.[1938], The large-sample distribution of the likelihood ratio for testing composite hypotheses, Ann. Math. Stat. 9 , 60-62.

[23] WITTING, H. and G. NÖLLE [1970], Angewandte Mathematische Statistik, Teubner, Stuttgart. 


\section{OTHER TITLES IN THE SERIES MATHEMATICAL CENTRE TRACTS}

A leaflet containing an order-form and abstracts of all publications mentioned below is available at the Mathematisch Centrum, Tweede Boerhaavestraat 49, Amsterdam-1005, The Netherlands. Orders should be sent to the same address.

MCT 1 T. VAN DER WALT, Fixed and almost fixed points, 1963. ISBN 906196 0029.

MCT 2 A.R. BLOEMENA, Sampling from a graph, 1964 . ISBN 9061960037.

MCT 3 G. DE LEVE, Generalized Markovian decision processes, part I: Model and method, 1964. ISBN 9061960045 .

MCT 4 G. DE LEVE, Generalized Markovian decision processes, part II: Probabilistic background, 1964. ISBN 9061960053.

MCT 5 G. DE LEVE, H.C. TIJMS \& P.J. WEEDA, Generalized Markovian decision processes, Applications, 1970. ISBN 9061960517.

MCT 6 M.A. MAURICE, Compact ordered spaces, 1964 . ISBN 9061960061.

MCT 7 W.R. VAN ZWET, Convex transformations of random variables, 1964. ISBN $906196007 \mathrm{x}$.

MCT 8 J.A. ZONNEVELD, Automatic numerical integration, 1964. ISBN 906196 0088 .

MCT 9 P.C. BAAYEN, Universal morphisms, 1964. ISBN 9061960096.

MCT 10 E.M. DE JAGER, Applications of distributions in mathematical physics, 1964. ISBN $906196010 \mathrm{x}$.

MCT 11 A.B. PAALMAN-DE MIRANDA, Topological semigroups, 1964. ISBN 906196 0118.

MCT 12 J.A.TH.M. VAN BERCKEL, H. BRANDT CORSTIUS, R.J. MOKKEN \& A. VAN WIJNGAARDEN, Formal properties of newspaper Dutch, 1965. ISBN 9061960134

MCT 13 H.A. LAUWERIER, Asymptotic expansions, 1966, out of print; replaced by MCT 54 and 67.

MCT 14 H.A. LAUWERIER, Calculus of variations in mathematical physics, 1966. ISBN 9061960207 .

MCT 15 R. DOORNBOS, Slippage tests, 1966. ISBN 9061960215.

MCT 16 J.W. DE BAKKER, Formal definition of programming languages with an application to the definition of ALGOL 60, 1967. ISBN 906196 0223.

MCT 17 R.P. VAN DE RIET, Formula manipulation in ALGOL 60, part 1, 1968. ISBN 9061960258 .

MCT 18 R.P. VAN DE RIET, Formula manipulation in ALGOL 60, part 2, 1968. ISBN $906196038 \mathrm{x}$.

MCT 19 J. VAN DER SLOT, Some properties related to compactness, 1968. ISBN 9061960266 .

MCT 20 P.J. VAN DER HOUWEN, Finite difference methods for solving partial differential equations, 1968. ISBN 9061960274 . 
MCT 21 E. WATTEL, The compactness operator in set theory and topology, 1968. ISBN 9061960282.

MCT 22 T.J. DEKKER, ALGOL 60 procedures in numerical algebra, part 1, 1968. ISBN 9061960290 .

MCT 23 T.J. DEKKER \& W. HOFFMANN, ALGOL 60 procedures in numerical algebra, part 2, 1968. ISBN 9061960304.

MCT 24 J.W. DE BAKKER, Recursive procedures, 1971. ISBN 9061960606.

MCT 25 E.R. PAERL, Representations of the Lorentz group and projective geometry, 1969. ISBN 9061960398.

MCT 26 EUROPEAN MEETING 1968, Selected statistical papers, part I, 1968. ISBN 9061960312 .

MCT 27 EUROPEAN MEETING 1968, Selected statistical papers, part II, 1969. ISBN 9061960401.

MCT 28 J. OOSTERHOFF, Combination of one-sided statistical tests, 1969. ISBN $906196041 \mathrm{x}$.

MCT 29 J. VERHOEFF, Error detecting decimal codes, 1969. ISBN 9061960428 .

MCT 30 H. BRANDT CORSTIUS, Excercises in computational Iinguistics, 1970. ISBN 9061960525.

MCT 31 W. MOLENAAR, Approximations to the Poisson, binomial and hypergeometric distribution functions, 1970. ISBN 9061960533.

MCT 32 L. DE HAAN, On regular variation and its application to the weak convergence of sample extremes, 1970. ISBN 9061960541.

MCT 33 F.W. STEUTEL, Preservation of infinite divisibility under mixing and related topics, 1970. ISBN 9061960614.

MCT 34 I. JUHÄSZ, A. VERBEEK \& N.S. KROONENBERG, Cardinal functions in topology, 1971. ISBN 9061960.622 .

MCT 35 M.H. VAN EMDEN, An analysis of complexity, 1971. ISBN 9061960630 .

MCT 36 J. GRASMAN, On the birth of boundary Zayers, 1971. ISBN 9061960649.

MLT 37 J.W. DE BAKKER, G.A. BLAAUW, A.J.W. DUIJVESTIJN, E.W. DIJKSTRA, P.J. VAN DER HOUWEN, G.A.M. KAMSTEEG-KEMPER, F.E.J. KRUSEMAN ARETZ, W.L. VAN DER POEL, J.P. SCHAAP-KRUSEMAN, M.V. WILKES \&. G. ZOUTENDIJK, MC-25 Informatica Symposium, 1971. ISBN 9061960657 .

MCT 38 W.A. VERLOREN VAN THEMAAT, Automatic analysis of Dutch compound words, 1971. 'ISBN 9061960738.

MCT 39 H. BAVINCK, Jacobi series and approximation, 1972. ISBN 9061960746.

MCT 40 H.C. TIJMS, Analysis of $(s, S)$ inventory models, 1972. ISBN 9061960754 .

MCT 41 A. VERBEEK, Superextensions of topological spaces, 1972. ISBN 90 61960762.

MCT 42 W. VERVAAT, Success epochs in Bernoulli trials (with applications in number theory), 1972. ISBN 9061960770.

MCT 43 F.H. RUYMGAART, Asymptotic theory of rank tests for independence, 1973. ISBN 9061960819.

MCT 44 H. BART, Meromorphic operator valued functions, 1973. ISBN 9061960827. 
MCT 45 A.A. BALKEMA, Monotone transformations and limit Zaws, 1973. ISBN 9061960835 .

MCT 46 R.P. VAN DE RIET, ABC ALGOI, A portable language for formula manipulation systems, part 1: The language, 1973. ISBN 9061960843.

MCT 47 R.P. VAN DE RIET, ABC ALGOL, A portable language for formila manipuZation systems, part 2: The compiler, 1973. ISBN 9061960851.

MCT 48 F.E.J. KRUSEMAN ARETZ, P.J.W. TEN HAGEN \& H.L. OUDSHOORN, An ALGOL 60 compiler in $A L G O L 60$, Text of the MC-compiler for the $E L-X 8$, 1973. ISBN $906196086 \mathrm{x}$.

MCT 49 H. KoK, Connected orderable spaces, 1974. ISBN 9061960886.

MCT 50 A. VAN WiJNGaARDEN, B.J. MAILLOUX, J.E.L. PECK, C.H.A. Koster, M. SINTZOFF, C.H. LINDSEY, L.G.L.T. MEERTENS \& R.G. FISKER (Eds), Revised report on the algorithmic language ALGOL 68, 1976. ISBN 90 6196. 0894.

MCT 51 A. HORDIJK, Dynamic programing and Markov potential theory, 1974. ISBN 9061960959.

MCT 52 P.C. BAAYEN (ed.), Topological structures, 1974. ISBN 9061960967.

MCT 53 M.J. FABER, Metrizability in yeneralized ordered spaces, 1974. ISBN 9061960975.

MCT 54 H.A. LAUWERIER, Asymptotic analysis, part 1, 1974. ISBN 9061960983.

MCT 55 M. HALL JR. \& J.H. VAN LINT (Eds), Combinatorics, part 1: Theory of designs, finite geometry and coding theory, 1974. ISBN 9061960991 .

MCT 56 M. HALL JR. \& J.H. VAN LINT (Eds), Combinatorics, part 2: graph theory, foundations, partitions and combinatorial geometry, 1974. ISBN 9061961009.

MCT 57 M. HALL JR. \& J.H. VAN LINT (Eds), Combinatorics, part 3: Combinatorial group theory, 1974. ISBN 9061961017.

MCT 58 W. ALBERS, Asymptotic expansions and the deficiency concept in statistics, 1975. ISBN 9061961025.

MCT 59 J.L. MIJNHEER, Sample path properties of stabie processes, 1975. ISBN 9061961076.

мСт 60 F. GÖBEL, Queueing models involving buffers, 1975. ISBN 9061961084 .

* MCT 61 P. VAN EMDE BOAS, Abstract resource-bound classes, part 1. ISBN 9061961092 .

* MCT 62 P. VAN EMDE BOAS, Abstract resource-bound classes, part 2. ISBN 9061961106 .

MCT 63 J.W. DE BAKKER (ed.), Foundations of computer science, 1975. ISBN 9061961114.

MCT 64 W.J. DE SCHIPPER, Symmetric closed categories, 1975. ISBN 906196 1122.

MCT 65 J. DE VRIES, Topological transformation groups 1 A categorical approach, 1975. ISBN 9061961130 .

MCT 66 H.G.J. PIJLS, Locally convex algebras in spectral theory and eigenfunction expansions, 1976. ISBN 9061961149. 
* MCT 67 H.A. LAUWERIER, Asymptotic analysis, part 2. ISBN $906196119 \mathrm{x}$.

MCT 68 P.P.N. DE GROEN, Singularly perturbed differential operators of second order, 1976. ISBN 9061961203 .

MCT 69 J.K. LENSTRA, Sequencing by enumerative methods, 1977. ISBN 9061961254 .

MCT 70 W.P. DE ROEVER JR., Recursive program schemes: semantics and proof theory, 1976. ISBN 9061961270 .

MCT 71 J.A.E.E. VAN NUNEN, Contracting Markov decision processes, 1976. ISBN 9061961297 .

MCT 72 J.K.M. JANSEN, Simple periodic and nonperiodic Lamé functions and their applications in the theory of conical waveguides, 1977. ISBN 9061961300 .

* MCT 73 D.M.R. IEIVANT, Absoluteness of intuitionistic Zogic. ISBN $906196122 \mathrm{x}$

MCT 74 H.J.J. TE RIELE, A theoretical and computational study of generalized aliquot sequences, 1976. ISBN 9061961319.

MCT 75 A.E. BROUWER, Treelike spacer and related connected topological spaces, 1977. ISBN 9061961327.

MCT 76 M. REM, Associons and the closure statement, 1976. ISBN 9061961351.

MCT 77 W.C.M. KALLENBERG, Asymptotic optimality of likelihood ratio tests in exponential families, ISBN 9061961343.

MCT 78 E. DE JONGE, A.C.M. VAN ROOIJ, Introduction to Riesz spaces, 1977. ISBN 9061961335 .

MCT 79 M.C.A. VAN ZUIJLEN, Empirical distributions and rankstatistics, 1977. ISBN 9061961459.

MCT 80 P.W. HEMKER, A numerical study of stiff two-point boundary problems, 1977. ISBN 9061961467 .

MCT 81 K.R. APT \& J.W. DE BAKKER (eds), Foundations of computer science II, part I, 1976. ISBN 9061961408.

MCT 82 K.R. APT \& J.W. DE BAKKER (eds), Foundations of computer science II, part II, 1976. ISBN 9061961416.

* MCT 83 L.S. VAN BENTEM JUTTING, Checking Landau's "Gmindlagen" in the automath system, ISBN 9061961475.

MCT 84 H.L.L. BUSARD, The translation of the elements of Euclid from the Arabic into Latin by Hermann of Carinthia (?) books vii-xii, 1977. ISBN 9061961483.

MCT 85 J. VAN MILL, Supercompactness and Wallman spaces, 1977. ISBN 9061961513.

* MCT 86 S.G. VAN DER MEULEN \& M. VELDHORST, Torrix I, ISBN 9061961521 .

* MCT 87 S.G. VAN DER MEULEN \& M. VELDHORST, TOYYix II, ISBN $906196153 \mathrm{x}$

MCT 88 A. SCHRIJVER, Matroids and Zinking systems, ISBN 9061961548 .

An asterisk before the number means "to appear". 
MCT 89 J.W. DE ROEVER, Complex Fourier transformation and analytic functionals with unbounded carriers, 1978. ISBN 9061961556 .

An asterisk before the number means "to appear". 
